NBSIR 85-3121

\title{
SOLID - A FORTRAN Program for Displaying Three-Dimensional Surface Topographies
}

David E. Gilsinn

U.S. DEPARTMENT OF COMMERCE National Bureau of Standards National Engineering Laboratory Center for Manufacturing Engineering Gaithersburg, MD 20899

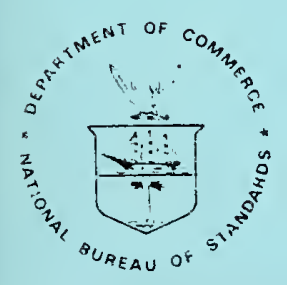

U.S. DEPARTMENT OF COMMERCE NATIONAL BUPEAU OF STANDARDS 

NBSIR $85-3121$

SOLID - A FORTRAN PROGRAM FOR

DISPLAYING THREE-DIMENSIONAL

SURFACE TOPOGRAPHIES

David E. Gilsinn

U.S. DEPARTMENT OF COMMERCE

National Bureau of Standards

National Engineering Laboratory

Center for Manufacturing Engineering

Gaithersburg, MD 20899

June 1985

U.S. DEPARTMENT OF COMMERCE, Malcolm Baldrige, Secretary NATIONAL BUREAU OF STANDARDS. Emest Ambler. Director 



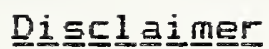

Certain commercial equipment, instruments, or materials ars identified in this paper in order to adequately specify the experimental procedure. Such identification does not imply recommendation or endorsement by the National Eureau of Standards, nor does it imply that the materials or equipment identified are necessarily the best available for the purpose. 

2.0 Program Dperation

2.1 Algorithm Dverview

2. 2 An Interactive Session

3.0 Three-Dimensional Geometric Considerations 32

3 I World Coardinate System

3.2 Devica Coordinate Eystem

उ.3 Viewer Coordinate System

3.4 Indesing the Light and Viewing Fays

3.5 Vector Representation of the Fay Vector Systen 37

S. 6 The Felation Between world Coordinates and Bay Coordinates

3.7 Projection of World Points to the viewing Window

40

3.8 Conversion from World Coordinates to Light or Viewing Coordinates

i. 7 Conversion of a Viewplane Foint to a World Coordinate Paint

3.10 Computing the Height Along a Fay

46

4.0 Discussion of Algorithms

51

4. 1 Shadowgraph Algorithm

4.2 The Solid Projection Algorithm

51

4. . Case Selection Table

55

4.4 Extreme Foint Selection Table

60

4.5 Entry Point Algoritim

60

4.6 Line Drawing Algorithm

63

65

5.0 Program Implementation

71

5. 1 System Commands

5.2 Main Program (SOLID)

71

5.2.1 Summary

5.2 .2 Flow Chart

5.2.3 Listing

72

72

7

91

5.3 Subroutine GTCLRS

5. $3 \times 1$ Summary

104

5.3.2 Flow Chart

5. 3. 3 Listing

S. 4 Subroutine SETCOL

5.4.1 Summary

5.4.2 Flow Chart

5.4.3 Listing

5.5 Subroutine GETFIL

5.5.2 Flow Chart 
5.6 Subroutine GETCHN

5.6.1 Summary

5.6 .2 Fiow Chart

5.6.5 Listing

119

5.7 Subroutine GNXY

5.7.1 Summary

120

5.7 .2 Flow Chart

122

5.7 .3 Listing

1.56

5. 8 Subroutine WFFIC

5. 9.1 Summary

5.8.2 Flow Chart

140

5.8.3 Listing

5.7 Subroutine RDPIC

141

5.9.1 Summary

5.9 .2 Flow Chart

142

142

5.9 .3 Listing

144

145

5.10 Subroutine EXTREM

5.10.1 Summary

5.10 .2 Flow Chart

5.10.3 Listing

154

5.11 Subroutine GETROWW

156

5.11 .1 Summary

5.11 .2 Flow Chart

156

5.11.3 Listing

157

158

5.12 Subroutine GETZ

159

5.12.1 Summary

5.12.2 Flow Chart

159

5. 12.3 Listing

160

161

162

5.13 Subrautine GETF

162

5. 13.1 Summary

5.13.2 Fiow Chart

165

5.13 .3 Listing

164

5.14 Subroutine GETV

165

5.14.1 Summary

165

5.14 .2 Flow Chart

160

5.14.5 Listing

167

5.15 Subroutine GETH

169

5.15 .1 Summary

168

5.15 .2 Flow Chart

169

5.15. 5 Listing

170

171

5.16 Subroutine GETXYZ

171

5.16.1 Summary

172

$\Xi=16.2$ Flow Chart

173

174

5.17 Subroutine WCASE

5.17.1 Summary

5.17 .2 Flow Chart

5.19 Subroutine XYIN

5. 18.1 Summary

5.18 .2 Flow Chart

5.18.3 Listing 
5.19 Subroutine FUTFIL

175

5.19.1 Summary

175

5.19 .2 Flow Chart

175

5.19.3 Listing

178

6.0 Acknowledgements

199

7.0 Bib1iography

200

Appendix A: Image Processing Functions

201

Appendix E: Systen Support Prograns

209

Appendix C: Image Data Acquisition

225 

The techniques for image analysis depend on the distribution of the "grey levels" or pixel intensity levels of a picture displayed on an image processor. Combinatorial, statistical or Fourier techniques can be used to find picture boundaries, adjust contrast or modify the picture in frequency space. An entirely different approach to picture eningncement is to look at the picture from a geometric point of $v i$ w and assume that the intensity value at a pixel represents a surface height, where brighter pixel intensities represent higher points and lower pixel intensities represent lower points. Then, if $a$ viewer looks at the picture from various perspectives, the geometric image displayed can yield interesting information from a visual perspective. This was the background notivation for writing this program. The simulated solid generated by the program on the display monitor is sometimes called a pseudosolid sirice a nonreal three-dimensional effect can be used to enhance a two-dimensionel picture such as a medical x-ray.

The program, documented in this manual, was modeled on an earlier program written by Dr. Roger L. Nagel of Lehigh University and discussed in Weber, Nagel [7]. This program is $\equiv$ redesign of Dr. Nagel's original code that takes advantage of the image processor features available to the author. Both programs implement an algorithm that generates a three dimensional solid image on the viewer"s screen by using a, technique that follows I ight rays from a light source to the refiecting surfare and then 
to the viewer"s eyes. This type of an algorithm is referred to as a ray tracing algorithm, see Goldstein, Nagel [5].

A large body of 1 iterature exists isee foley and Van Dan [4]) describing computational techniques to display the imeges of three dimensional objects by a) eliminating.hidien surfaces, b) applying shading or c) casting shadows. Most of the techriques require initial processing of the displayed scone. This usually requires that the program user describe in fairly great detail the geometric structure of the scene in such a manner that the objects in the scene can be decomposed into adjoining polygons. These techniques, however, become very unwieldy when the scene displayed is very rough, such as a very mountainous area. It is this situation that a user encounters when displaying the texture of machined metal parts. For this reason a point-by-point shadowing technique was used, see Appel [1].

The problem addressed by the progam documented here is as follows: Given a randomly rough surface, such as a machined metal surface, we assume that the topography of that surface has been digitized in such a way that the digital scale is proportional to the surface height. The specific technique used to acquire surface topographic maps is briefly described in Appendix C. Furthermore, we assume that the digitized points are distributed in such a way that they form an evenly spaced square grid. Each record in the digitized data file represents an amplitude trace across the surface with data values taken at, say, N evenly spaced paints. There are $N$ records in the data file. We wish to first simulate the illumination of the surface 
in order to highlight the rough topography and then to view the surface from various orientations.

This problem lends itself to the ray tracing techniqus. It does not require prepracessing of the data file in order to establish connectivity relations since they are inherent in the data file and the mode of acquisition. Ray tracing is a procedure that defines the light intensity at every point on the monitor screen by tracking a ray of 1 ight from the 1 ight source to the rough surface and then to the monitor as if it were a viewing window.

The ray tracing algorithm used in this program is a two-pass algorithm, see Crow [2], in the sense that the surface data file is processed twice, first to generate shadows and then to construct a solid projection of the illuminated surface. In the first pass the object surface is divided into two classes of areas, those that are shadowed and those that are not. The result is displayed on the user monitor in a form called a shadowgraph. The effect shown simulates a viewer looking down on a scene with light illuminating the surface from some specified direction. In the second pass of the algorithm the hidden surfaces reiative to the viewer are removed and the resulting image is projected onto a window thett represents the monitor screen. The advantage of this two-pass approach is that the shadow generation is separated from the picture generation process. This allows the viewer to look at the surface from various angles without changing the light source.

This manual approaches the documentation from the point of view of refinement in the sense that as one proceeds through this 
document, one begins with a global picture as a user in section 2. This section also includes an interactive scenario. Next, from the point of view of the analyst, the document discusses in more detail the analytic geometry and algorithms that are implemented in the programs. This is contained in Sections 3 and 4. respectively. Section 5 includes 1 istings of the main program and subroutines along with their flow charts. Finaliy, the appendices broadly describe 1) the general architecture of the image processor on which the algorithm was implemented, 2) the image processor specific and host system subroutine calls required and 3$)$ the $3-0$ stylus data profilometer used to acquire the data.

The author feels strongly that a documentation of this rature serves a useful purpose. A fundamental myth must be abandoned by those who think that using a standard language on the host computer makss transportability possible. It is true that, since the algorithm was implemented in FORTRAN, the program could be transferred by tape and most 1 ikely compiled on another system. Ary connection with portability stops there. The architecture and controlling software for image processors are all different. No standards for interfacing host driven software and image procsssor hardware exist. The implementation of any algorithm becomes an ad hoc exercise in communicating a mathematically described algorithm, by way of a possibly standard ianguage, through specialized non-standard control prograns to a unique device. Although an algorithm may be stated in a general form, the implementation of that algorithm on a specific device 
or combination of devices is usually a nontrivial undertaking. The author feels that it is worthwhile for those both familiar and unfamiliar with implementing graphics algorithms to see how a general algorithm is tailored to a particular system. The author hopes that the detailed discussion of the algorithm will encourage others to modify this program or rewrite it as necessary in order to implement the algorithm on another system. Dunham [ذ] has alsด voiced similar sentiments. 


\subsection{Algorithm Qverview}

Since the nature of the process used to generate a solid is accomplished in two applications of the same essential algorithm the program was structured in such a manner that the user can run it from the beginning to the final soiid generation or terminate it after creating a shadowgraph. In either case the program assumes that a surface topographic image is available as a sequential file with 512 records of 512 bytes each. Furthermore, the progran assumes that the image processor being used is enabled and that the display monitor is on.

The topography of a surface can be interpreted as a grid of impulse spikes with amplitudes ranging from 0 to 255 . This image is limited by the hardware only. Each spike is referred to as a pixel or picture element. It is displayed on the monitor as a dot, whose intensity is controlled by the values 0 to 255 . 0 is the lowest intensity (black) and 255 is the highest (bright white). The entire topography when stored in the image processor is sperified by 512 \& 512 dots. The intensity of the dots represent the digitized amplitude of the surface at that point. The higher the point, the higher the intensity. The lower the surface point, the lower the intensity. For a discussion of the digital data acquisition techniques used to acquire surface topographies, the reader is referred to the Appendix $C$.

If a shadowgraph of an image is not available then one must be created. This is done on the first pass. Once the program initializes the image processor, it asks the user for the file 


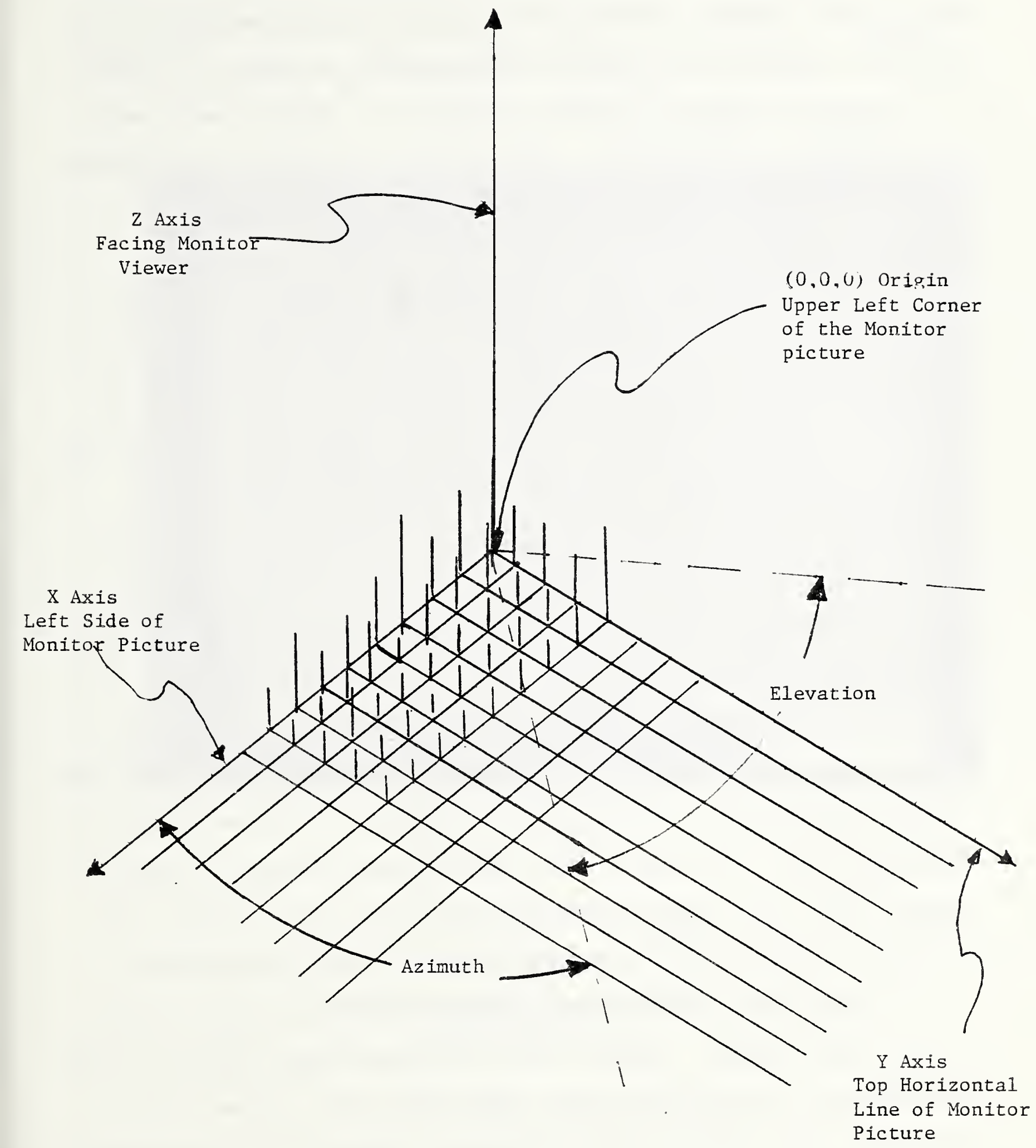

Figure 1

Conceptual Picture of a

Topographic Map 
name of the topographic image. The program displays the image on the graphics monitor and asks the user for the azimuth and elevation angles of the desired light source as shown in figure 1. A typical surface is shown in Figure 2. This is what would be displayed on the monitor. The surface in Figure 2 represents approximately $1 \mathrm{~mm} \times 1 \mathrm{~mm}$ of stainless steel with a roughness height of about $1 \mu \mathrm{m}$.

In the geometric model used for this progran the image is assumed to form a solid within a box (called the bounding box) of sides 511 units in length (512 grid points per side) and height 255 units (256 grid points in height). The 256 height grid points represent the intensity levels for the image processor. The origin of the right hand coordinate system, referred to as the world coordinate system, 1 ies at point $(0,0,0)$ of the box in Figure 3. The origin is sometimes referred to as 0 . Feferring to Figure 3 , consider the vector $0 \vec{A}$. The azimuth angie, $A Z$, of the vector $O \vec{G}$ is measured from the positive $X$ axis in $a$ counterclockwise manner. The elevation, EL, is measured from the XY-plane verticaliy. In Figure 3 , if the point A were taken as (511, 511, 255), the angle, EL, would be approximately 19.5 degrees. For most cases the elevation of the 1 ight source used is usuaily greater than 20 degrees and less than 90 degrees.

The idee behind the general algorithm used in this program is relatively simple. Every line in the Euclidean 3-space can be represented by an equation that associates the $\bar{L}$ value on the line with $\equiv$ pair $(X, Y)$ on the $X Y$-plane. These $(X, Y)$ Values fali on a Iine that is the projection of the line in space onto the xY-plane. This 1 ine is called the S드므 linge. As one steps along 
Origin $(0,0)$

$(0,511)$

$\mathrm{Y}$ Axis

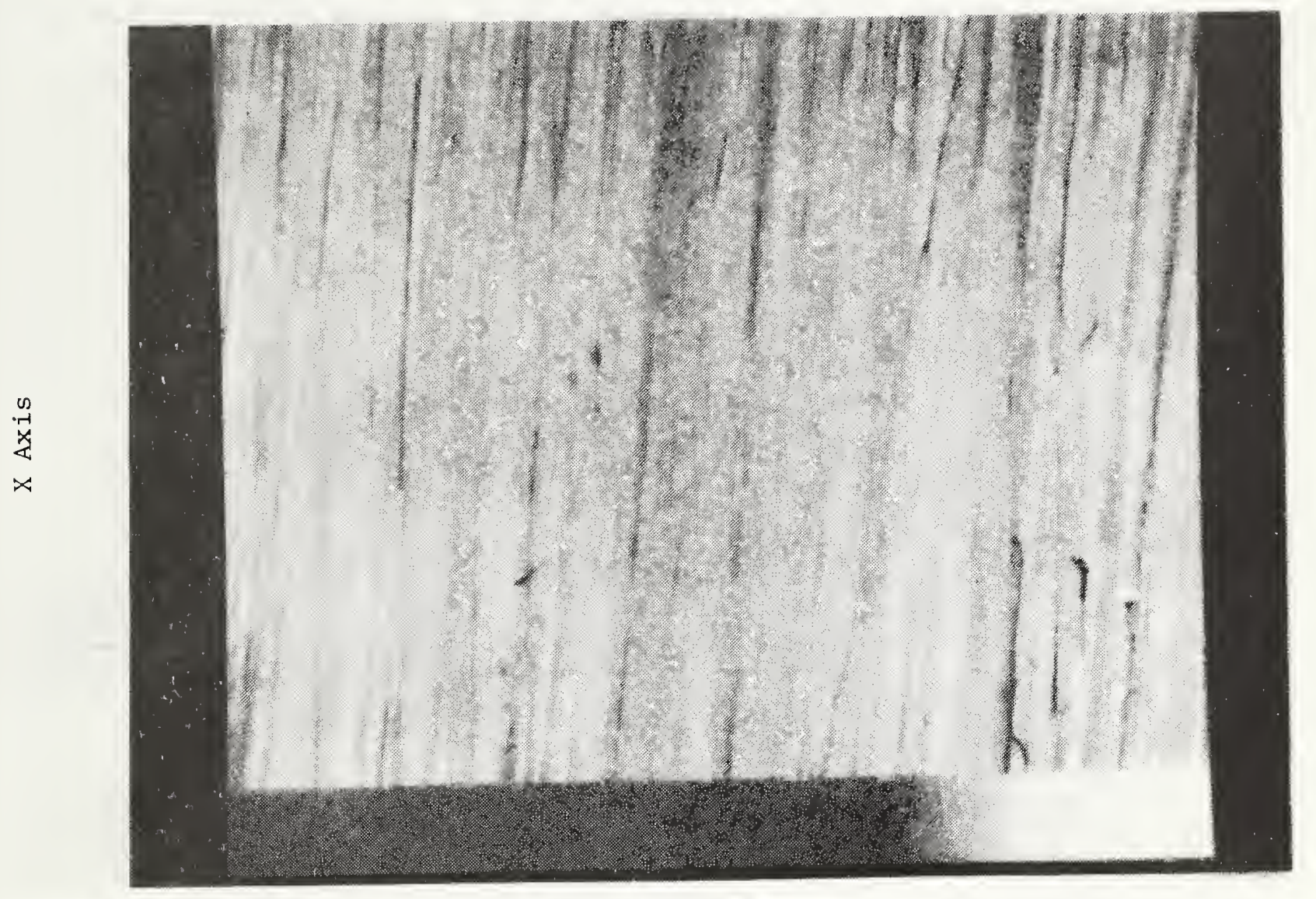

$(511,0)$

$(511,511)$

Figure 2

A Surface Topographic Image with

Grey Level Bar 


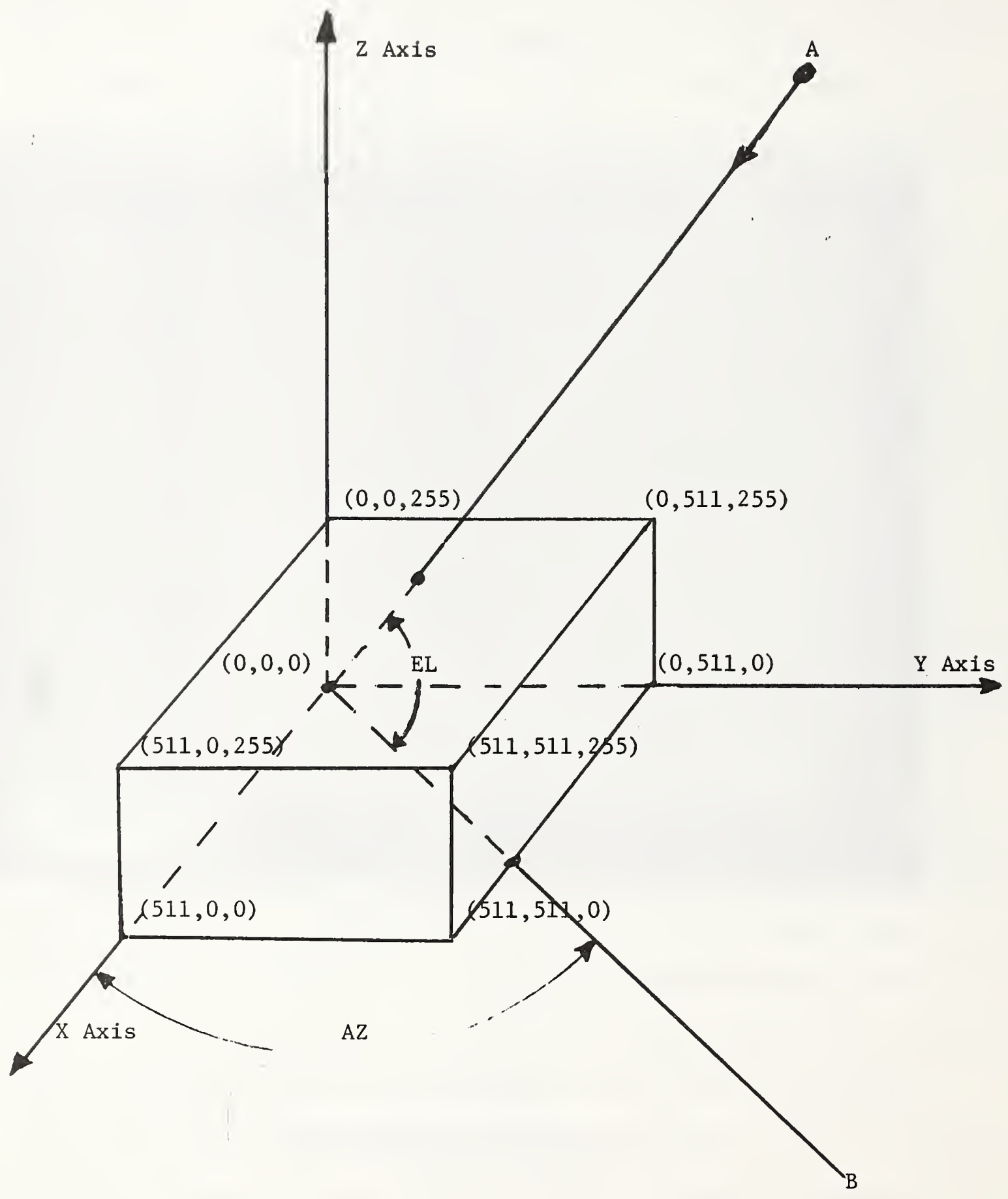

Figure 3

Bounding Box with Azimuth and Elevation Shown 
the scan line, one can always find the associated value $Z$ in Epace. Therefore, the Iine in space can be "traced" by sequentially selecting $(X, Y)$ points along the scan 1 ine.

In order to trace rays by computer from a source to the topographic surface some bound must be put on the set of all rays in order to establish program 1 imits for those rays that will be traced to the surface. Consider Figure 4. Assume that the 1ight source lies at infinity so that all light rays are parailel. Let the azimuth and elevation of the light source be $A z$ and EL: respectively, as already shown in Figure 3 . Notice that the oriy possible rays that could impinge on the surface 1 ia between the two planes $F_{1}$ and $F_{2}$. These are vertical planes intersecting the XY-plane at the lines $L_{1}, L_{2}$, where $L_{1}$ and $L_{2}$ are 1 ines through the points EX1 and EX2 in Figure 4. These points will be called Extreme Roints. $L_{1}$ and $L_{2}$ are parallel to the projection of the

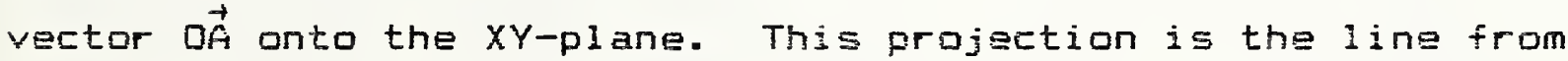
$(0,0,0)$ to $B$ in Figure 3 . Dnce the azimuth and elevation are given, the program can look up the extreme points from $a$ prespecified table. These are then used as bounds on where the program begins and ends.

Qnce the extreme points for the image have been identified ray tracing from the light source to the surface can begin. The procedure traces rays beginning at extreme point Ex1 lp planes paraliel to $P_{1}$ and $P_{2}$ movirig incrementaily to a new $p l a n e ~ P$ after each cycle of the shadowgraph portion of the algorithm is completed. The program stops when extreme point Ex2 has been encountered. On each plane $P_{;}$rays are traced beginning with the 


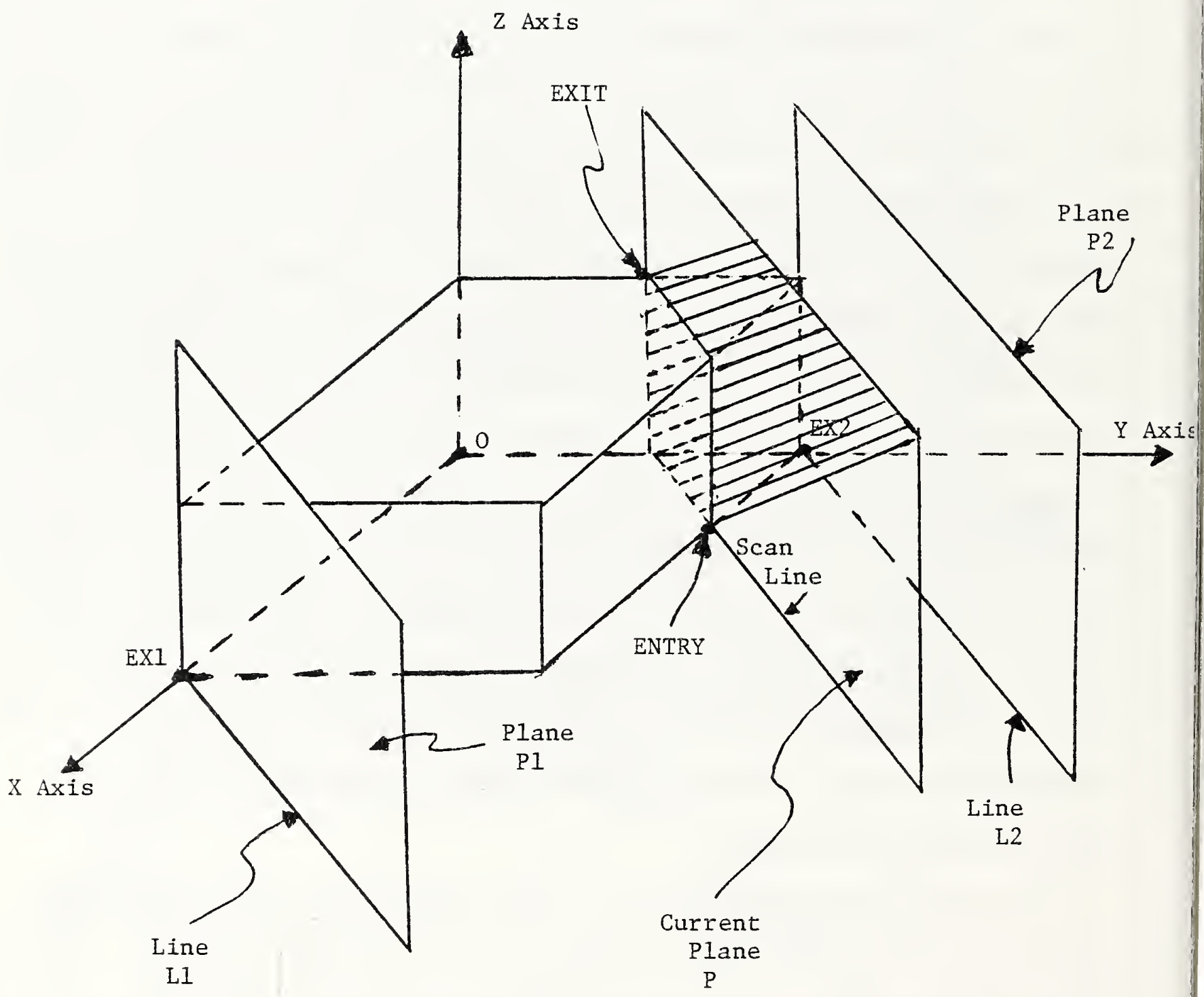

Figure 4

Extreme and Current Planes 


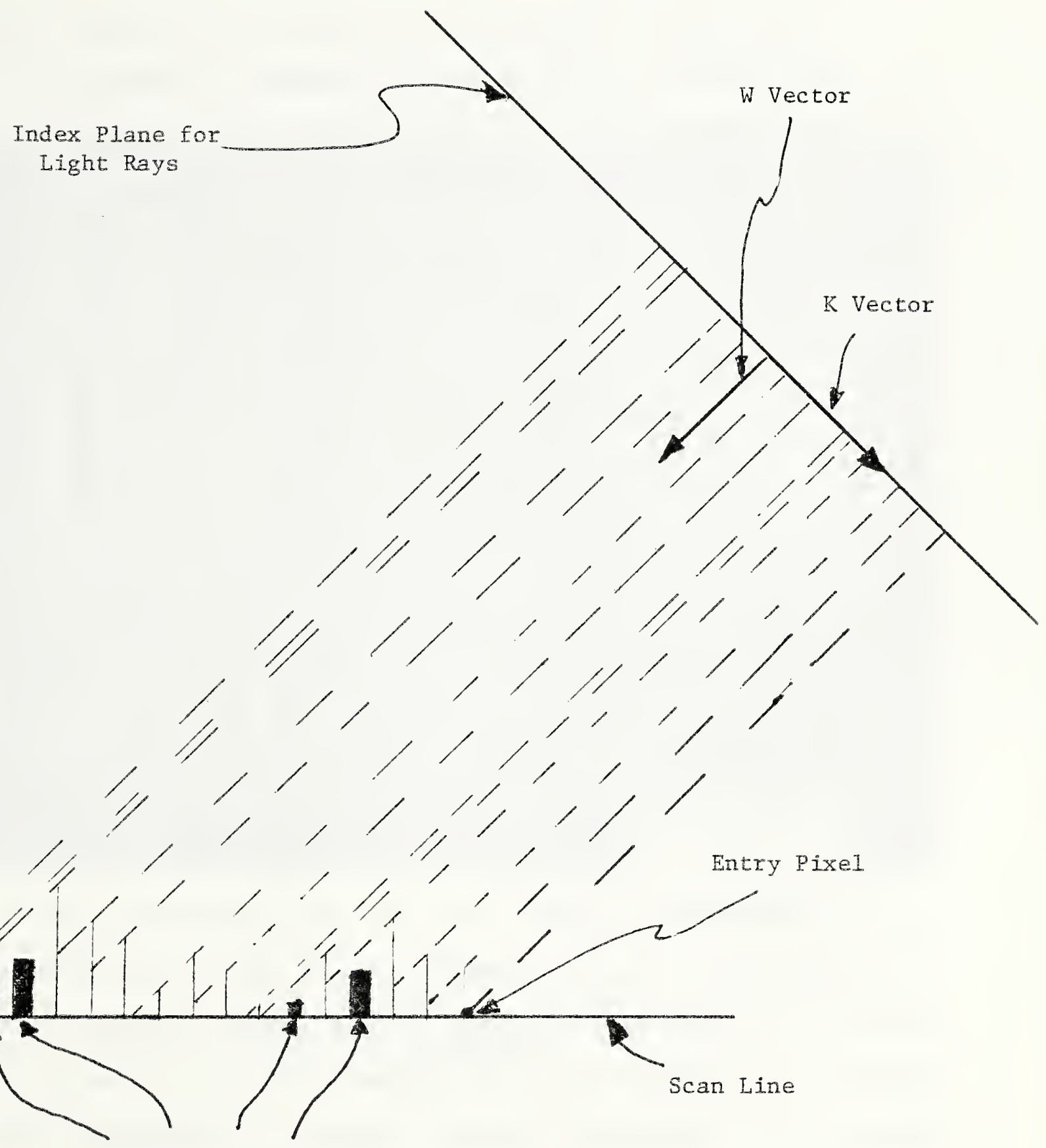

Shadowed Pixels

Figure 5

Geometry for the

Shadowgraph 
one passing through the ENTRY point in Figure 4 and terminating with the one through the EXIT point.

In each plane $P$ parallel to $F_{1}$ and $P_{2}$, rays are traced from the 1 ight source to a point on the curface. Feferring to Figure 5, if the height of a pixel falls below a ray then nothing is entered into the shadowgraph map, which is displayed as the host program creates it that is, a black pixel is displayed. If the height of the pixel is greater than or equal to the ray height, the original pixel value is written to the shadowgraph. The set of all points whose values fall below all rays traced are those in shadow. The shadowgraph for a sample calculation, with $A Z=45$ and EL=75, is given in Figure 6 . The black areas of the picture are the points of the original image that fali within the shadow. The shadowgraph then is a second image that, along with the original image, is used to shadow the solid image in the second part of the program. Dnce the shadowgraph has been generated the user may save the image, if desired, before proceeding to generate a solid.

in generating a solid the user can designate a portion of the shadowgraph for solid generation by using the interactive trackbali and function buttons of the image processor. The trackball is used to move the screen cursor in order to specify

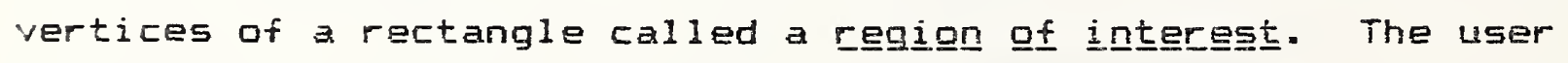
then enters the azimuth angle and elevation angle for the viewer rays and a percentage value used to reduce the intensity of those pixels designated for shadowing. From experience a reasonabie number is $45 \%$. 


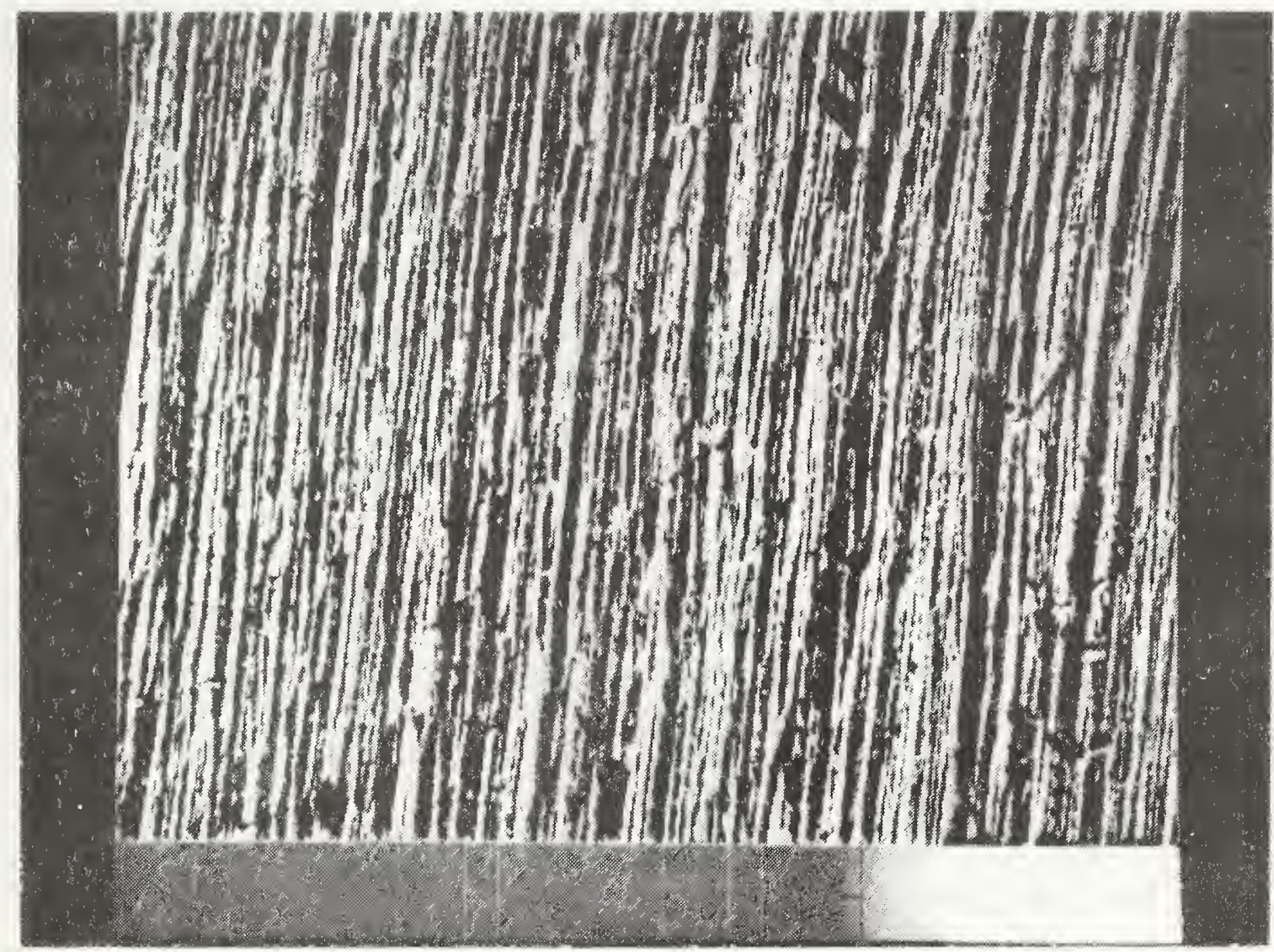

Figure 6

Sample Shadowgraph 
From the azimuth and elevation angles the viewer frame of reference is generated. The viewer frame of reference, represented by unit vectors $\vec{K}, \vec{w}$, and $\vec{k} \times \vec{W}$ in Figure 7 , is an orthonormal set of vectors used to trace points along rays from the viewer's eyes to the surface and to index points on the viewing screen. The screen can be considered a viewport opening in an extended plane in front of the viewer called the vịewpla Given the selected rectangular area (region of interest) of the shadowgraph and the viewer coordinate system, the extreme points are selected, as in the shadowgraph pass of the algorithm. The viewing screen is indexed by the two coordinate vectors, $\vec{k}$ and $\vec{k} \times \vec{W}$. $\vec{k}$ points vertically down the screen and $\vec{k} \times \vec{W}$ points to the user"s right. The program computes the multiples of the $\vec{k} \times \vec{w}$ vector that project down to the extreme points. These multiples are added to the projection of the center of the region of interest onto the viewplane. They are used to index planes F1, F'2 as in Figure 4. These planes bound the computations.

For the sake of terminology, vertical lines on the screen are referred to as collumns and horizontal lines as rows. The program starts at the column containing the left most projection of an extreme point and then moves to the next column. In earh column it processes pixels from the bottom of the screen to the top. The column it works on is called the curegnt column. The program first finds the initial viewscreen row for that current column. Then the row is selected that is the projection of the entry point of the viewing rays into the solid, see Figure 4. Since each pixel falls on a screen row, that row is referred to as the current row. 


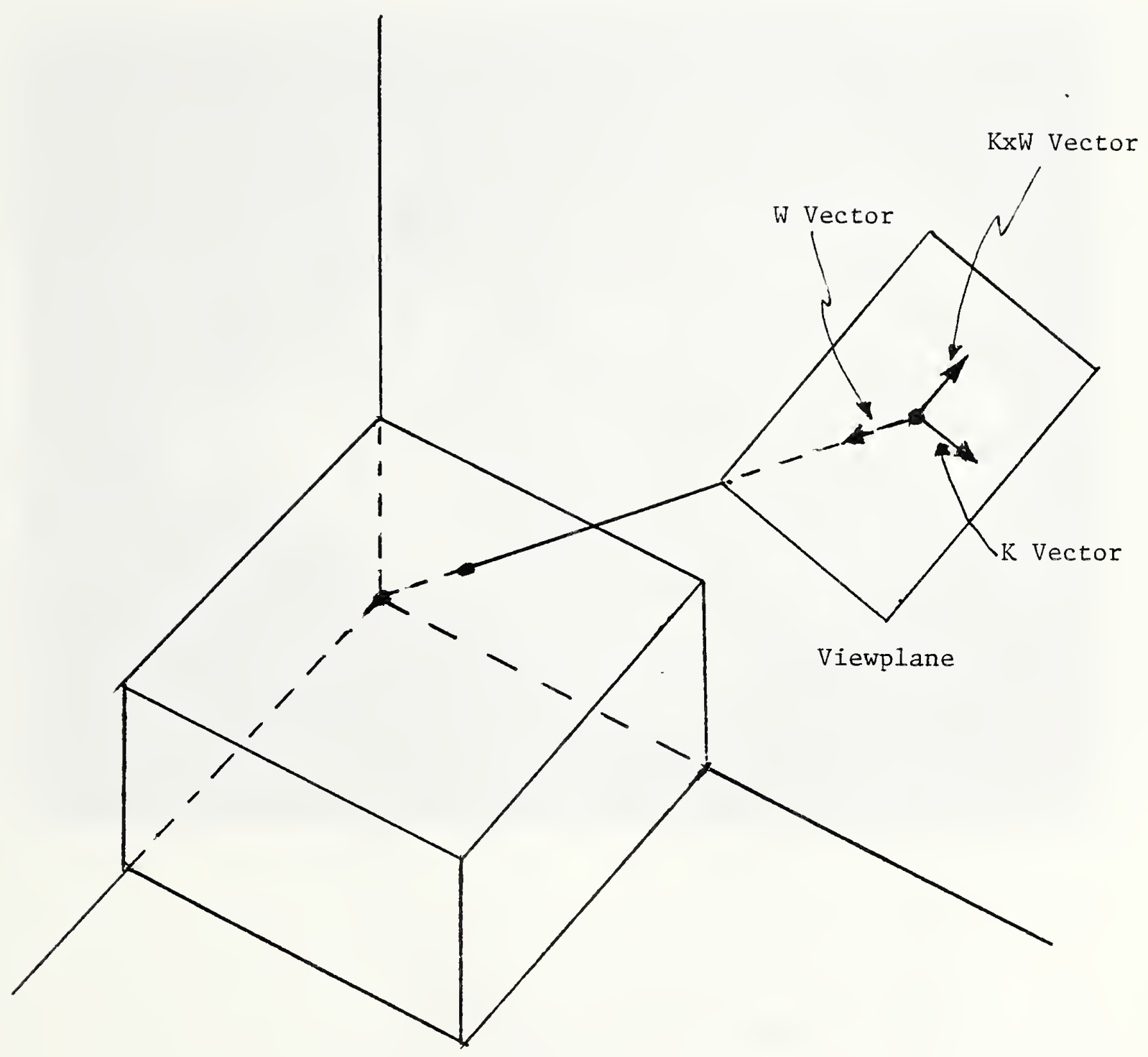

Figure 7

Viewplane Coordinate

System 


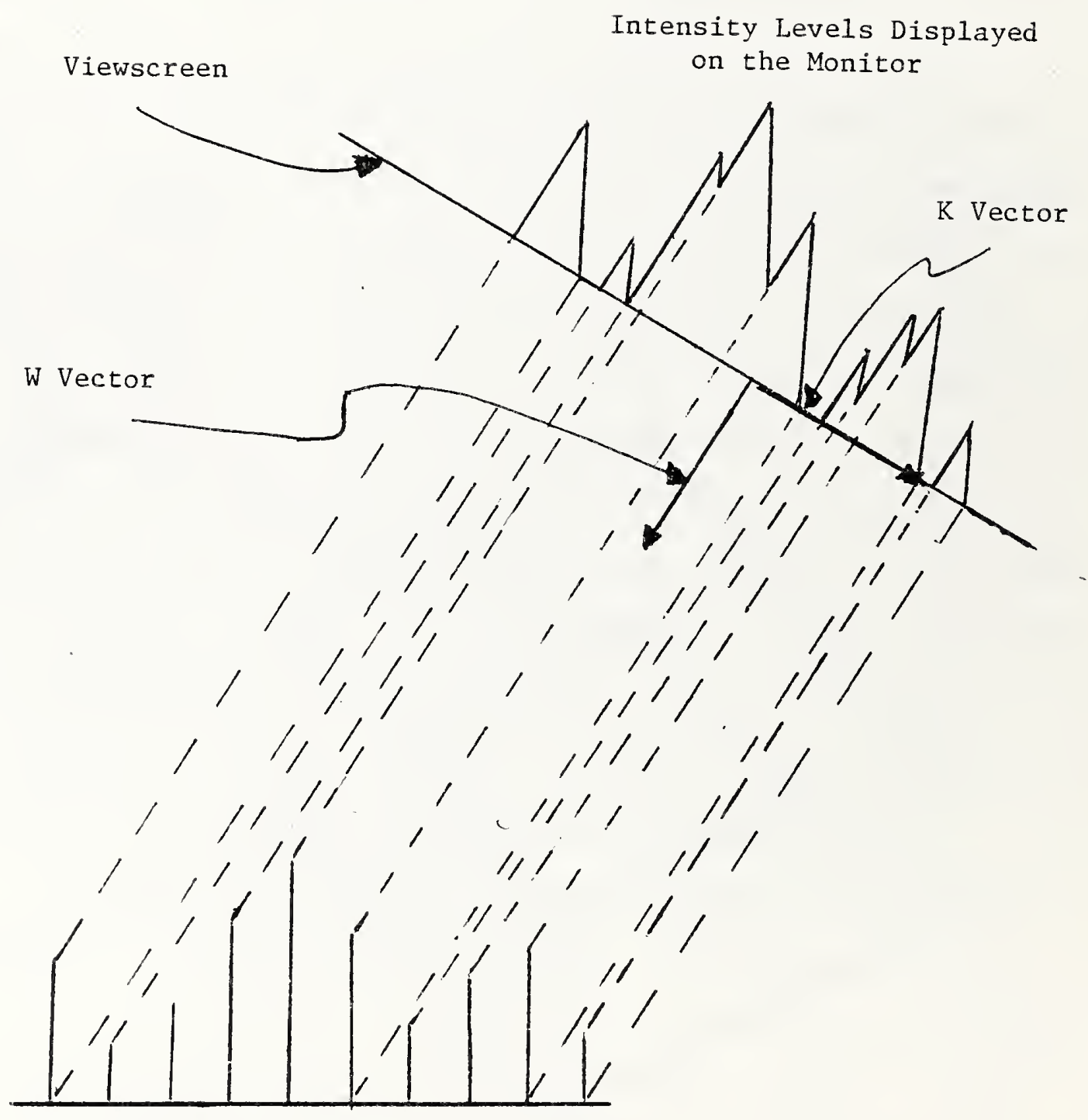

Topographic Data Set Intensities

Figure 8

Correspondence

of Displayed Intensities to

Surface Amplitude Values 


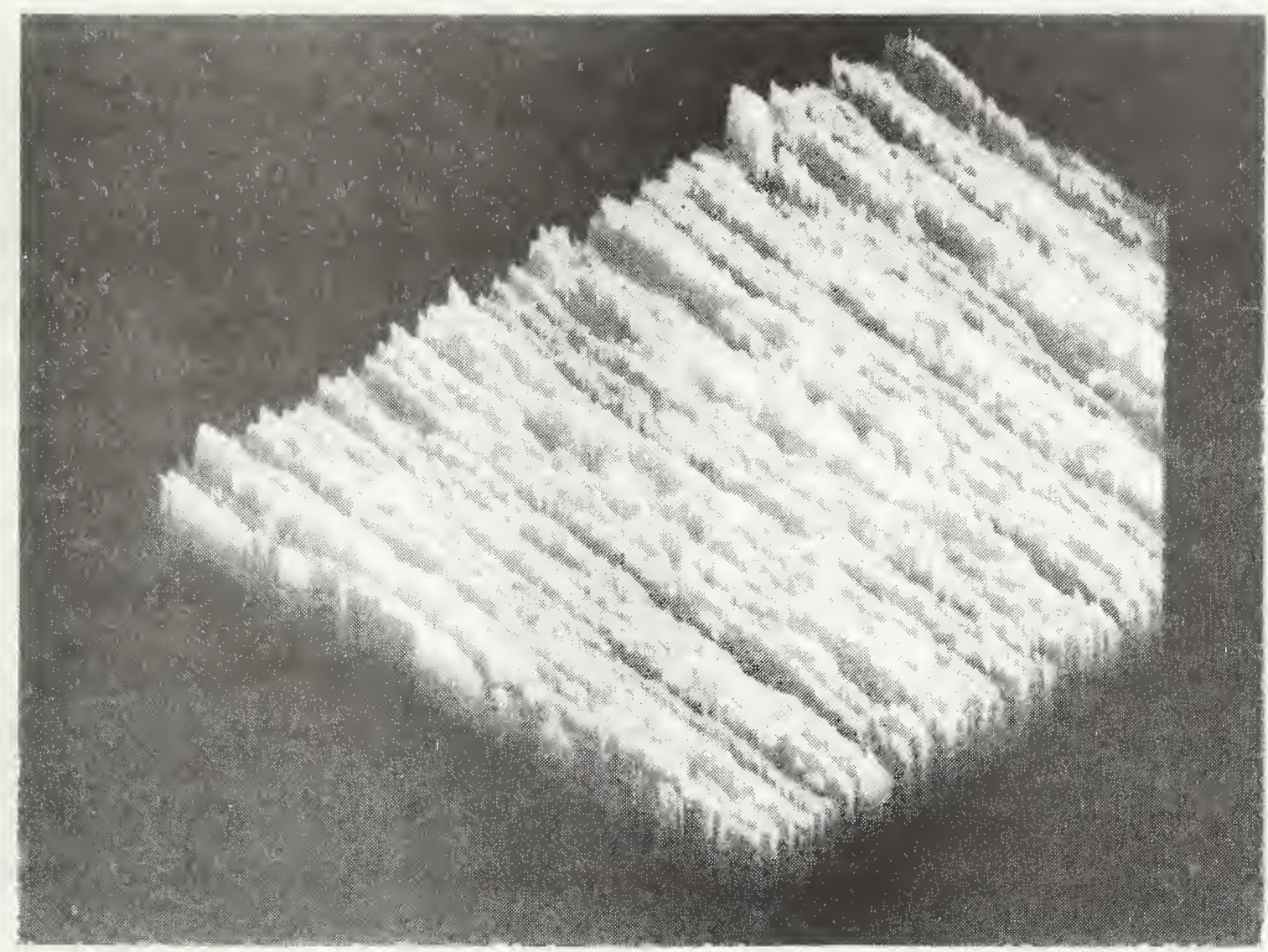

Figure 9

Projected Image of a

Surface Sample 
For the sake of a mental picture, one can think of image pixels as spikes sitting above the base $X, Y$-plane (Figure 1 ). The height of each spike represents the intensity value of the image pixel. From the point of view of a spectator looking at the solid from an angle, more than just the top of the spike is seen, as would be the case if one were looking directly down on the image. The first ray, selected for processing, is that which encounters the base point of a pixel sitting at the boundary of the solid area. The program then traces rays through each pixel in the current column until it hits the point representing the top of the boundary pixel. The intensity of the points displayed represent the height of the viewing ray above the XY-plane when it encounters the spike representing the pixel, see figure 8. This is why at the boundary the intensities in figure 9 rise in value from 0 to the full value of the pixel. If the pixel 1 ies in a shadow e.g. the darker section of the projected surface in Figure ?, all of the values displayed for that spike are reduced by the selected percentage. The right hand side of figure 9 shows the computer processing a column vertically.

Once a ray misses the top of a pixel in a column it is traced along until it hits another pixel spike. The height of the ray at that point is then written to the screen. The process continues up the column until a ray leaves the area selected. This is shown in Figure 8.

After a column has been processed from bottom to top the program maves to the next column on the right and starts at the bottom again. This continues until that collumn is reached that 
contains the projection of the second extreme poirt. This is the 1 ast solumn processed.

\section{2 An Interactive Session}

In this section the user is introduced to the interactive dialogue used by the program. Before beginninga the user is assumed to have read privileges for the desired image data file. which consists of 512 logical records, each consisting of 512 contiguous bytes.

Assume then that the user has signed on and verified access to the required data file and the host system has returned the prompt character. On the host system used by the author, this is an *. The user enters SOLID followed by a carriage return. It wouid look like this

\section{*SOLID<CRY}

where CCF stands for the non-printing character for carriage return. This calls a user created command file that icads the program task, assigns the appropriate peripheral devices to the job, and then starts the job.

The program first prints

IF YOU WISH TO SHADOW A PICTURE TYPE 0. IF YOU WISH TO CREATE A PSEUDO-SOLID TYFE 1. * *NDTE: TO FSEUDO-SOL ID AN IMAGE A SHADOUGFAPH MUST HAVE PREVIOUSLY BEEN CFEATED.

followed by a program prompt character. For the author's system this is a character. The program user must enter something at this point.

Assume that a shaciowgraph does not exist. Then the user types a 0 and a carriage return. It would look ike this 
$30<C R$

where o represents the zero. The program then prints

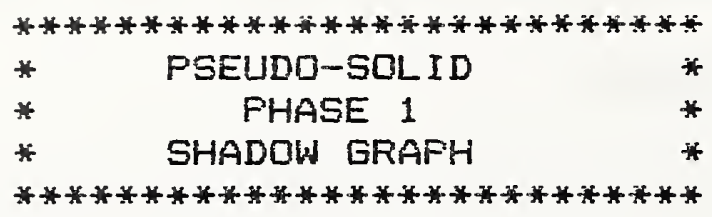

THIS PORTION DF THE FSEUDO-SOLID GEMERATION SIMLLATES THE EFFECT OF A DISTANT SOUFCE DF LIGHT SHINING ON THE SURFACE. THOSE AREAS DF THE SUFFACE THAT WOULD BE SHADED ARE DARKENED. NO THREEDIMENSIDNAL EFFECT IS CREATED IN THIS PART.

THE MAIN PICTURE IS HRITTEN TO CHANNEL 1 AND THE SHADOW GRAPH IS GENERATED ON CHANNEL 2.

This is fallowed by

ENTER NAME OF IMAGE FILE YOU WISH TRANSFERRED MAX OF 16 CHAR: $>$

Assume for the sake of this exampie that an image resides on a disk with disk name IMG: and the image file is SUFFACE.DAT. Then after the $>$ the input would look $1 \mathrm{ike}$

\section{YMG: SURFACE. DATSCRY}

At this time the host would transfer the image to refresh memory 1 of the image processor. For a description of the general architecture of the image processor used, see Appendix 1 . After the picture has been transferred, the host returns the nessage

IF THE PICTLRE HAS BEEN PROPERLY GENERATED, TYFE 1, DTHERWISE O TD GET ANDTHER FICTURE. $>$

If the user types o, the hast asks for the file nane again. If 1 is entered as in

\section{>CCF?}

the program next asks for the azimuth and elevation angles of the 1 ight source. See Figures 1 and 4. 


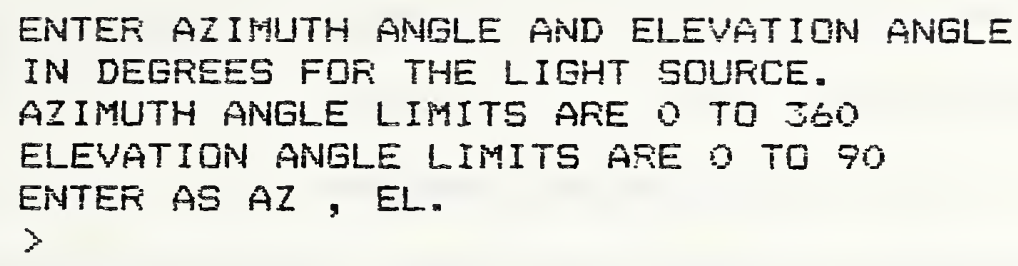

Suppose, for example, that the user wouid like to shadow the supface with an azimuth of $4 \bar{y}$ degrees and elevation of 75 degrees, then the input sequence woul of look like

$$
>45 ., 75 .<C R \text { \% }
$$

If the user enters any value outside of the limit, the message and prompt will appear again. If the user enters the first value and misses the second the host ordinarily will return with the prompt >, expecting the second value.

As soon as these data values have been entered the jost and image processor start generating the shadowgraph. In the case above, the user would see tracing beginning on the monitor at the left hand lower corner and proceed along the diagonais beginning at the bottom of the right hand side and tracing to the left or top side at a 45 gegree angle. Figure ó is the resulting shadowgraph for Figure 2 .

After the shadowgraph has been generated the user is given an option to save the shadowgraph with the message IF YDU UISH TO SAVE THIS SHADDWGRAFH TYFE 1. DTHERWISE $D$.

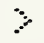

If the user enters 1 , such as

$$
>1<C R Y
$$

the program returns the message

ENTER THE NAME OF THE FILE YOU WISH TO CFEATE. MAX GF $1 S$ CHARACTEFS. 
to which the user would supply a file name with extension. SHw, to designate a shadowgraph file; in the form

\section{YIMG: SURFACE. SHWKCR >}

The program then enters the second pass of the algorithm. In this part a projection of a selected portion of the image is generated on the viewplane. If at the beginning of the program the user had selected to bypass the shadowgraph generation the following message is written. It is also written after the shadowgraph is generated.

IF THE OFIGINAL PICTURE IS IN CHANNMEL 1 AND ITS SHADOWGRAPH IS IN CHANNEL 2 THEN TYPE 1 DTHERWISE TYPE O TO TRANSFER THE FICTLIRES. $>$

If a shadowgraph previously exists and the user wishes to generate a solid image projection then enter 0 in the form $30<C R>$

If O has been seiected then the following is printed ******** LOADING ORIGINAL IMAGE ********

foll owed by

ENTER NAME OF IMAGE FILE YOU WISH TFANSFERRED, MAX OF 1O CHAR. y

At this point the user types the image data file name and the file is transferred to the image processor and displayed on the monitor. The program then prints the nessage IF THE PICTURE HAS BEEN FFDPERLY GENERATED, TYFE 1, DTHERWISE O TO EET ANDTHER FICTURE. $>$

If a 1 is typed, this message is followed by ******** LOADING THE SHADOWGRAFH

foil owed by 
ENTER THE NAME DF IMAGE FILE YOU WISH TRANSFERRED, MAX OF 16 CHAR.

$>$

Here the user must enter the shadowgraph file name for the image displayed on the monitor. After the image is transferred it ramains visible on the monitor and the message

IF THE SHADOWGRAFH HAS BEEN PROPERLY GENERATED, TYFE 1, OTHERWISE O TO GET APOTHEF SHADOWGFAFH. $>$

appears on the user console. If the user types 1 then the program moves to an interactive mode in which the user must identify a rectangular region of interest on the shadowgraph that will be used to project a solid onto the display monitor. The user interacts with the image processor by way of a trackball with function buttons. For an illustration of the system configuration see figure 10. The first message printed on the user cansole is

$* * * * * * *$ IDENTIFY THE REGION FOR PSEUDO-SOL ID
******** ENHANCEMENT BY USING THE TRACKBALL.

THE LSER MUST IDENTIFY TWO DIAMETRICALLY OFFOSITE CORNERS OF A RECTANGLE USING THE TRACKBALL BUTTOHS. MOVE THE CLRSDR WITH THE TRACKBALL TO THE FIFST CORNER OF THE RECTANGLE OF INTEFEST. FUSH EUTTON A.

The user then selects the upper left corner of the desired rectangle with the cursor by way of the traclbali. After selecting the point the user presses button $A$ on the trackball housing. Once the processor has selected the point the host camputer displays the message

NOW MOVE THE CURSOF TO THE DIAMETFICALLY OFPOSITE CORNER OF THE RECTANGLE DF INTEREST. FUSH BUTTON A. Aftar moving the cursor by way of the trackball to the diametrically opposite corner of the desired rectangle the user 


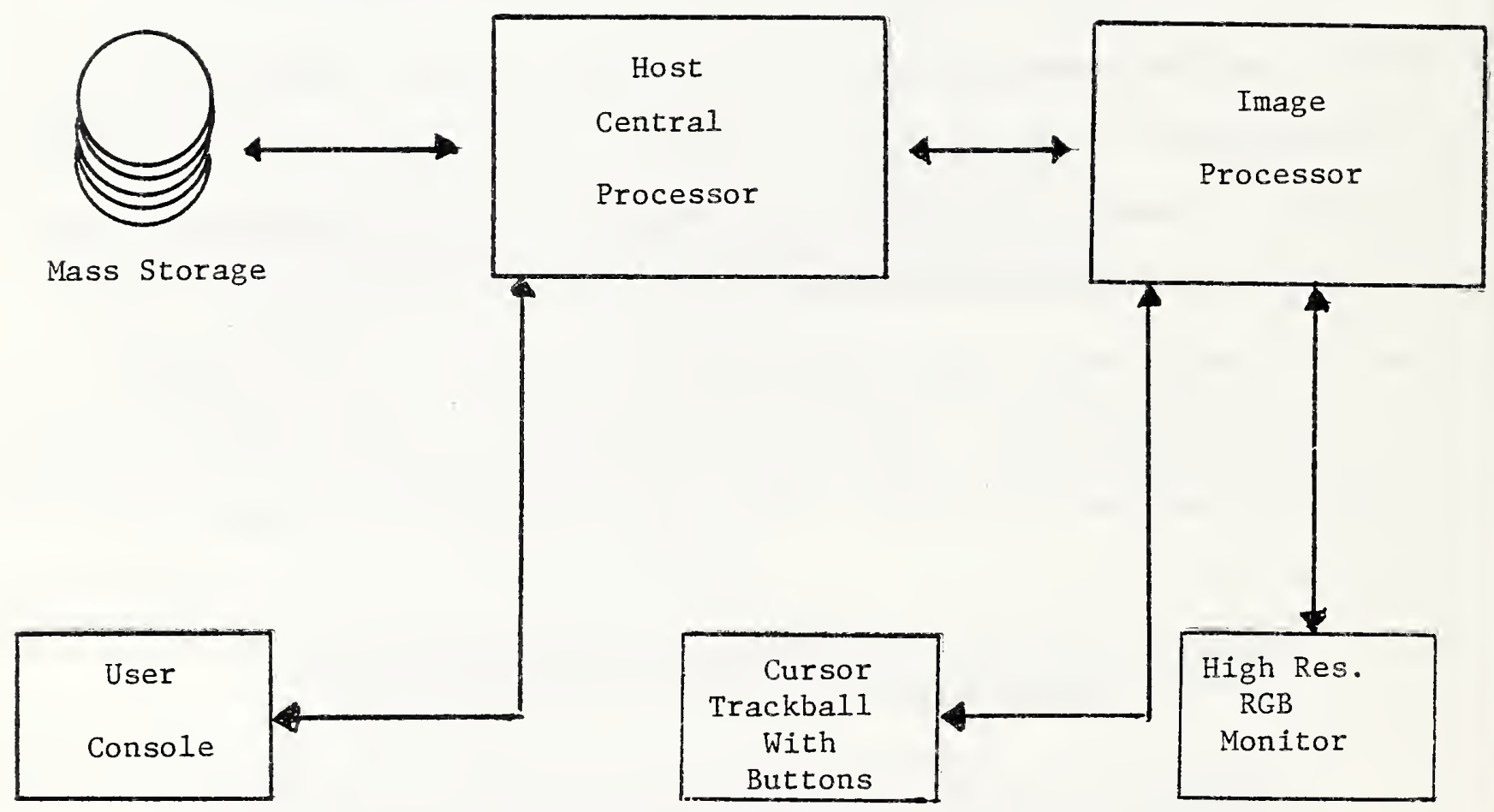

Figure 10

System Configuration 
again pusines button $A$. The image processor then outlines the rectangle selected by drawing boundary lines and places a pius sign in the center to indicate the central point that the viewer will be seeing when the solid is prajected. If the user has made an error in selecting the rectangle and wishes to select a new rectangle the processor allows this with the message

IF YOU WISH TO CHANGE YOUF MIND ON THE FECTANGLE OF INTEREST FUSH BOTTON B, OTHEFWISE PUSH BUTTON A

Assuming that the user presses button $A$, the host then prints the message

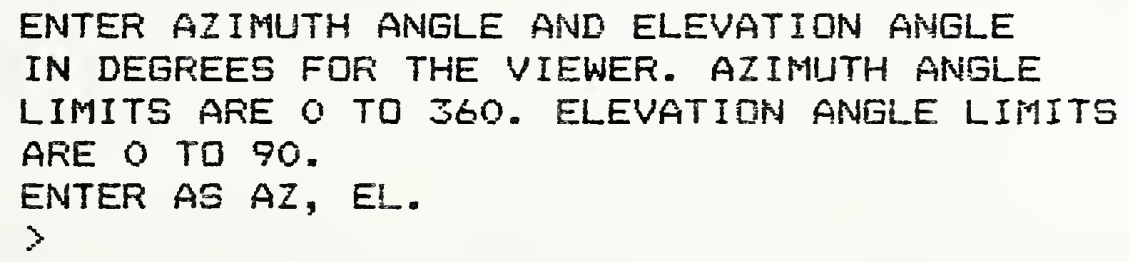

If a user wishes to view the solid from an azimuth of 15 degrees and elevation angle of 75 degrees, the following would be entered

$$
\times 15,75 ., \mathrm{CR}
$$

The host computer then returns with

ENTER THE FEFICENT REDUCTION IN INTENSITY DESIFED FOR SHADONING. ENTER FROM O. TO 100. $>$

Since the viewer will in general look at a surface from $\exists$ direction ather than that of the iight source, some of the points seen would normally fall into shadow. From ordinary experience areas that are shadowed, say by trees or houses, are still visible but with reduced intensity. The reduced intensity comes from any diffuse lighting of the scene. In order to simulate this effect the user can enter a percentage value that will be 
used by the prograin to reduce the intensity of pixels seen by the viewer but are cast into shadow. From user experience percentage values of 40 to 45 percent reduction give an adequate shadow simulation. Therefore for a 45 percent reduction the user would enter

$$
\text { >45. } \angle \mathrm{CF}>
$$

The program immediately starts generating the solid projected image moving from left to right on the screen tracing vertically from bottom to top. Some sample salids are shown in Figures 11 and 12. These pictures represent two views of the same region of interest of the surface in Figure 2. In particular, the region of interest is a portion.of the upper right quadrant of the picture.

At the end of the solid generation the program prints

IF YDU WISH TO SAVE THE PSEUDOSDLID IMAGE TYFE 1, OTHERWISE O $>$

If the user types 1 then the message ENTER THE NAME OF THE FILE YOU WISH TO CREATE, MAX OF 16 CHARACTERS. $>$

appears after which the user types the file name desired followed by the extension. Sol to indicate that this is a solid image, as for example, IMG: SLRFACE. SOL. This is followed by a carriage return. The following message appears IF YOU WISH TO GENERATE ANDTHER SOLID TYPE 1 , QTHERWISE O

If the user types o the program terminates, and if the user types 1 the same shadowgraph will be used and the processor prints the next message to the user console 
IF YOU WANT THE SAME REGION-DF-INTEREST TYPE 1, OTHERWISE O

$y$

If the user types 1 , then the same rectangle as earlier outlined would be used but the user can look at it from a different viewpoint by selecting a new viewing azimuth and elevation. If a o is entered, the program returns to the shadowgraph and allows the user to select a new rectangle for sol id projection.

Frocessing then continues as before. 


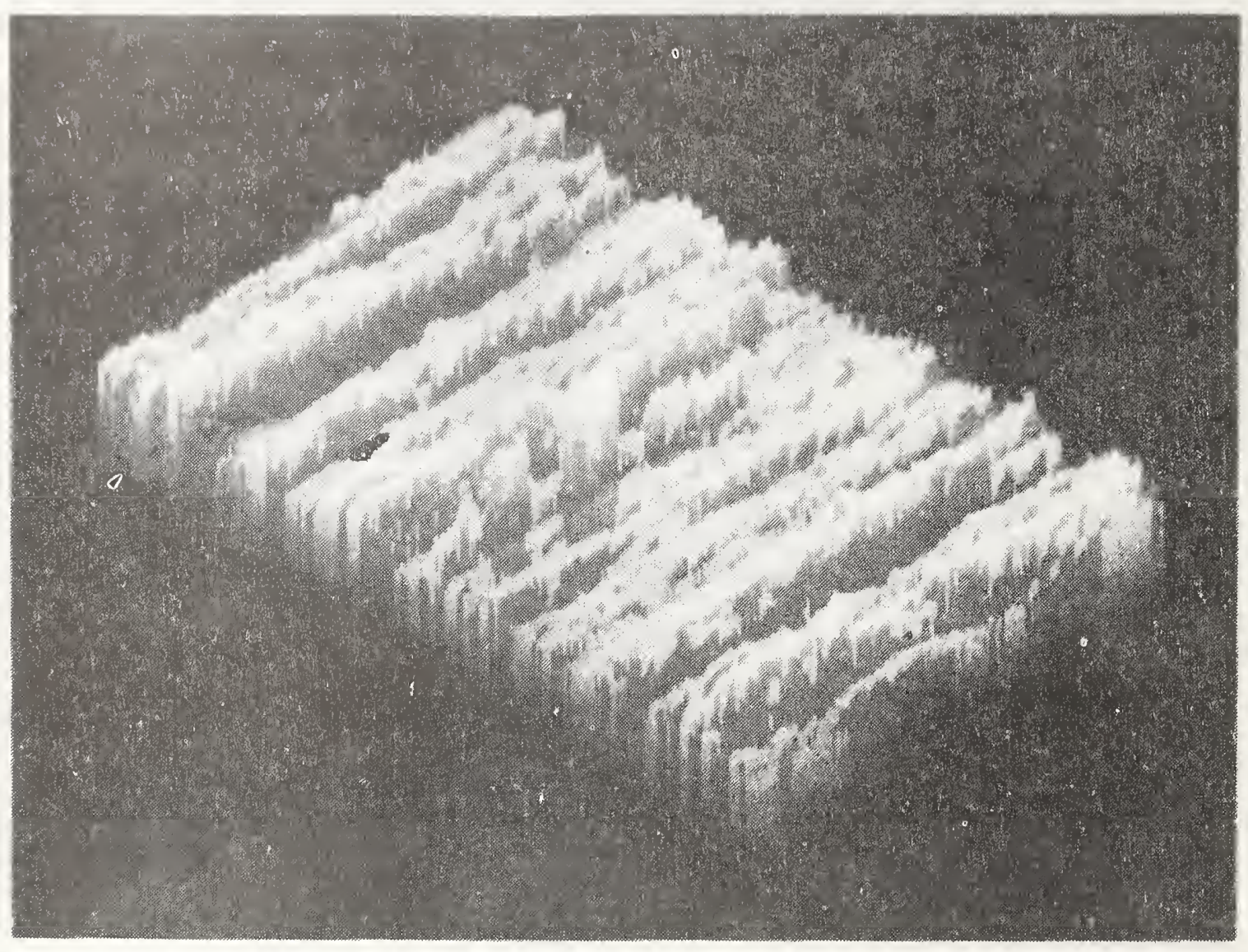

Figure 11

Solid Projection of a Portion

of the Upper Right Corner of Figure 2 


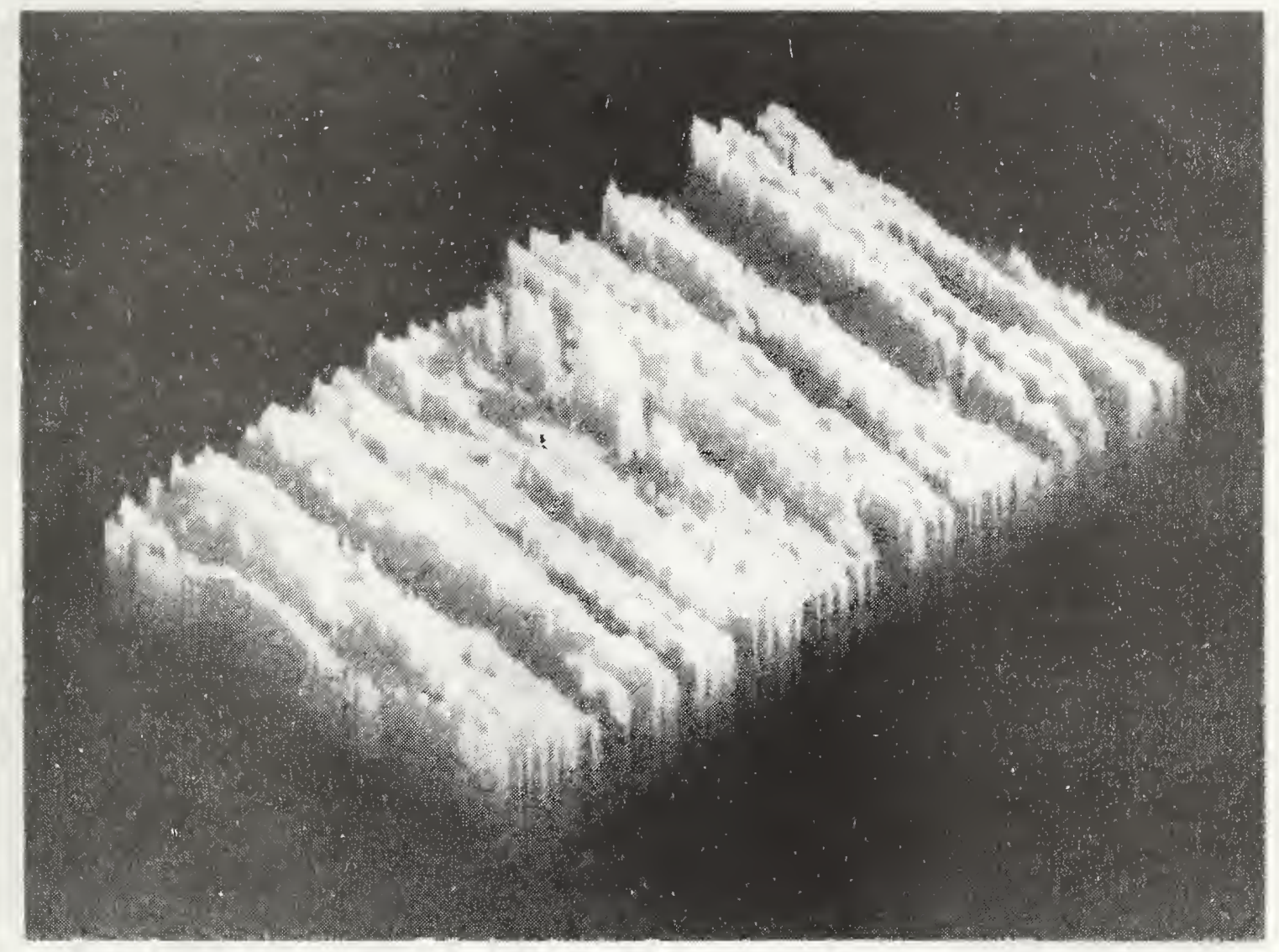

Figure 12

Figure 11 Rotated 180 Degrees 


\section{O Three-Dimensianal Geometric Considerations}

In this section, the necessary vector geometry techniques will be described. The mathematical tools developed will be used in the graphics algorithms to locate points in three-dimensional space in such a way that they can be uniquely traced to points on a viewer's screen. This requires defining special coordinate systems and linking them properly.

\section{1 Wor 1d Coordinate System}

The application or user oriented coordinates are generally referred to as wor $\underline{1} \underline{\underline{d}}$ 드므므므ㅁㅡㅡㅡㄹㅗ. The world coordinate system in this application will be a right-handed three-dimensional

Cartesian coordinate system. For a surface image the world coordinate system will be placed so that if a person were looking straight down on the top of the surface as in Figure 2 the origin would appear in the upper left hand corner. The positive world coordinate $X$-axis would then point vertically downwards and the positive world-coordinate $Y$-axis would point horizontally to the right. The positive world-coordinate Z-axis would point directly at the viewer. See Figure 13 . The $Z$-axis units represent digitized intensity levels of $0-255$, lower values represent low intensities, the XY-coordinate ranges are $0-511$.

\subsection{Device Coordinate Space}

The user of image processors must be aware of their device"s specific coordinate system. Thus, for example, in the image processor used the coordinate system used on the device reverses 


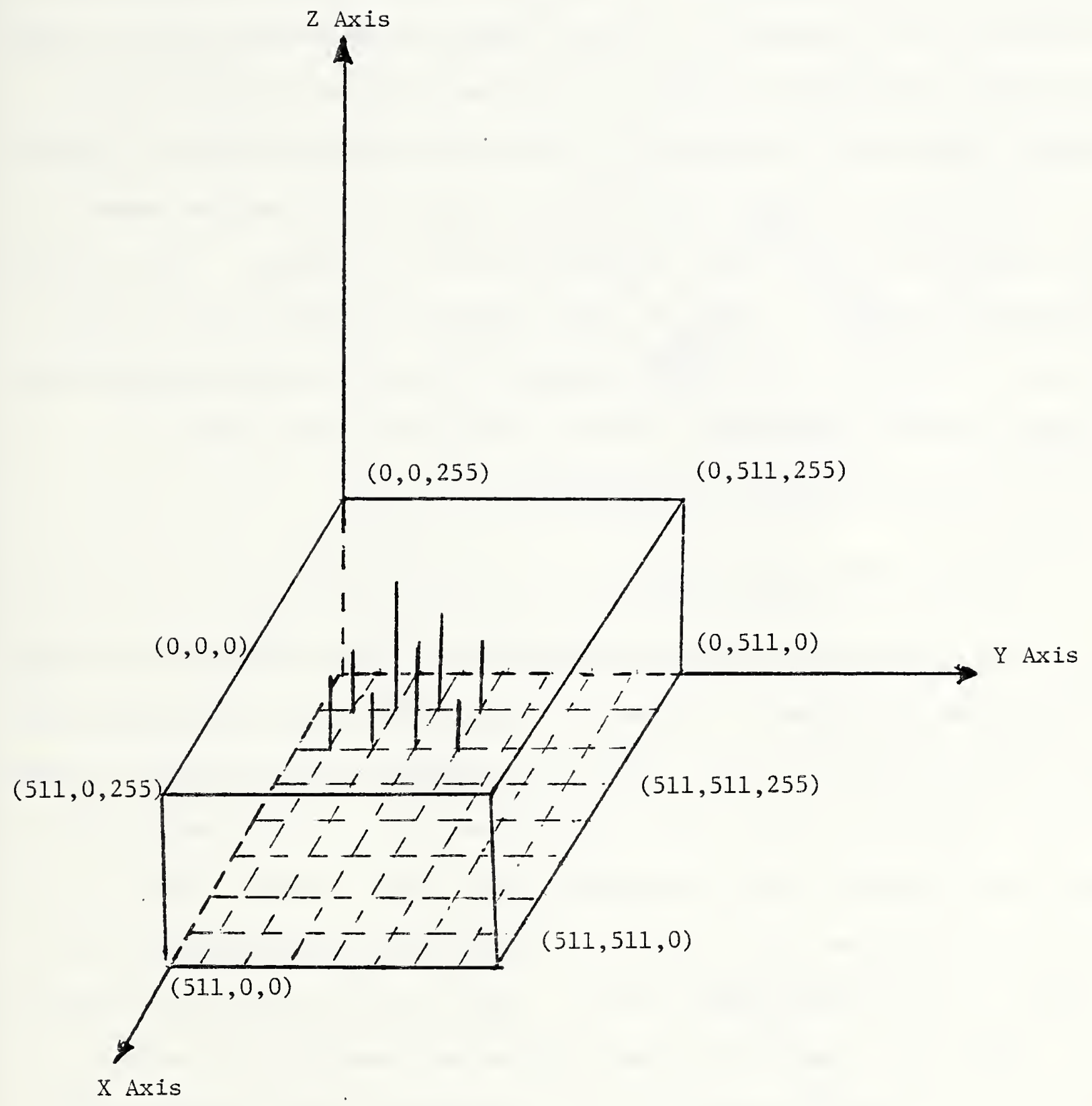

Figure 13

World Coordinate System 
the $X$ and $Y$ axes so that the device $X$-axis is the world coordinate $Y$-axis and the device $Y$-axis is the world coordinate x-axis. This confusion is overcome sometimes by calling points along the world coordinate $Y$-axis sample indices and points along the world coordinate $x$-axis as traverse indices. This device systen comes about because the image processor performs a raster scan from the left to right and top down of the refresh memory; the same way a television screen picture is scanned. This device coordinate system is used in many graphics systems and can lead to some confusion. We shall attempt to use the world coordinate system defined throughout and note the differences when explaining the software references.

\subsection{Viewer Coordinate System}

The general approach to generating a three-dimensional image used in this program is to define a portion of three-dimensional space and project it onto the viewing screen. The viewing screen can be thought of as a window to the world. A two-dimensional conrdinate system can be constructed on this window. The coordinate system that identifies points on this window will be called the $V-H$ coordinate system. With the $v-H$ system defined on the viewpiane, specifying the minimum and maximun vit values defines the viewing wirdow in the viewplane. The viewplane is orthogonal to the viewing rays to the surface. viewing rays can be thought of as 1 ines aiong which viewers sight as they look at an object. The portion of the world projected onto the window is called the yiㅡw yollume. In the present case, since orthogonal 


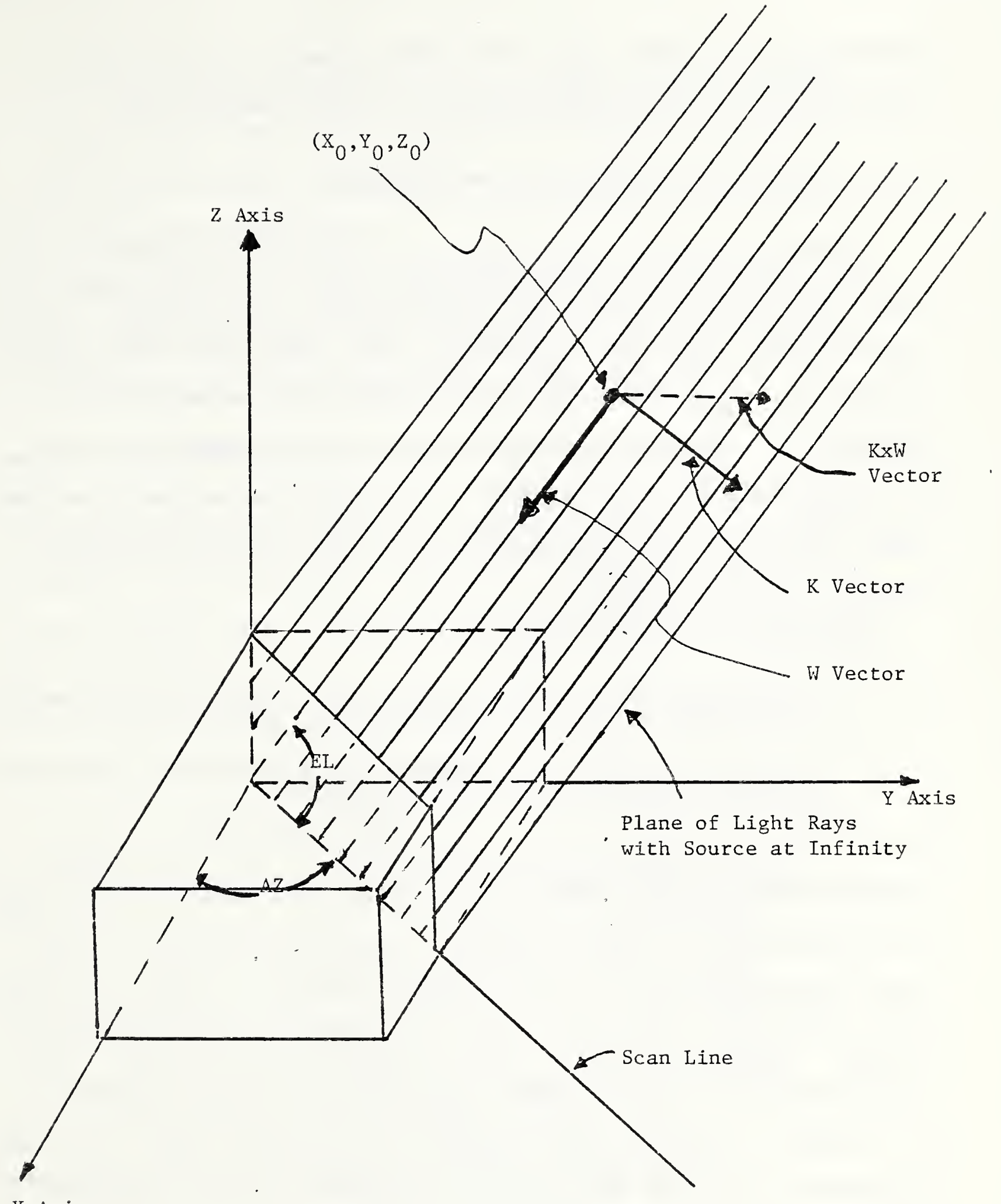

Figure 14

Light Ray Coordinates 
projections are being used, the view volume is an infinite parallelepiped with sides parallel to the viewing rays.

\subsection{Indexing the Light and Viewing Rays}

Assume that the 1 ight source is a point at infinity and all rays impinging on the surface are parallel. See figure 14 for an illustration. Let the direction of the 1 ight source be given by two angles, an azimuth and an elevation. The azimuth $A Z$ is measured in a positive sense beginning at the positive $x$ axis. It ranges from 0 to 360 degrees. The elevation angle EL of the light source is measured upwards from the $X Y-p l$ ane and falls between 0 and 90 degrees.

\section{Now set up two unit vectors:}

1) $\vec{W}$ - This unit vector points along the light rays and toward the origin.

2) $\vec{k}$ - This unit vector is orthogonal to $\vec{W}$ and points downwards across a plane made of 1 ight rays.

We use the same terminology as that used for the 1 ight source because the algorithm used is essentially the same for the light and viewing rays. Given an azimuth and an elevation for the light source one can think of a plane formed by rotating the xZplane by the azimuth angle. Now fill up this plane with light rays that point in the direction of the W-vector. Consider a unit vector in this plane, called $\vec{k}$, orthogonal to $\vec{W} . \vec{k}$ is then orthogonal to al $i$ of the light rays pointing in the direction $\vec{w}$. Each point on a fixed light ray in the plane can be indexed from a fixed point on the light ray by adding some multiple of the vector $\vec{w}$. Each light ray can be indexed from a fixed point on the plane by adding some multiple of $\vec{k}$. Finally, all light rays 
in the direction $\vec{w}$ fall on some plane parallel to the rotated plane. If one takes the cross product of $\vec{k}$ and $\vec{w}$ one gets $a$ vector that can be used to access any plane parallel to the rotated $p l a n e$, as in Figure 14.

This same procedure can be used to define viewing rays. In this latter case, $\vec{K}$ and $\vec{K} \times \vec{w}$ index points on the viewing plane. This is orthogonal to the viewing rays, indexed by $\vec{w}$.

\subsection{Vector Representations of the Ray Vector System}

Let CE be the cosine of the elevation angles. CA the cosine of the azimuth, SE the sine of the elevation angle, and SA the sine of the azimuth angle. Then $\vec{w}, \vec{K}$, and $\vec{k} \times \vec{w}$ can be represented in vector triple form as

$$
\begin{aligned}
\vec{w} & =(-C E * C A,-C E * S A,-S E) \\
\vec{K} & =(C A * S E, S A * S E,-C E) \\
\vec{K} \times \vec{w} & =(-S A, C A, 0)
\end{aligned}
$$

where * is multiplication. These are developed as follows:

1) Refer to Figure 15 for $\vec{w}$. From simple formulas the distance from $A$ to $B$ is -SE since $\vec{W}$ has unit langth. The magnitude of the length from $A$ to $O$ is CE. Then the length from $D$ to $A$ is $-C E * S A$ and from $C$ to $A$ is -CE*CA. The components of $\ddot{w}$ are then (-CE*CA, -CE*SA, -SE). Noie that $\vec{W}$ as constructed is a unit vector since

$$
\begin{aligned}
(-C E * C A)^{2}+(-C E * S A)^{2}+(-S E)^{2} & =C E^{2}\left(C A^{2}+S A^{2}\right)+S E^{2} \\
& =C E^{2}+S E^{2} \\
& =1 .
\end{aligned}
$$




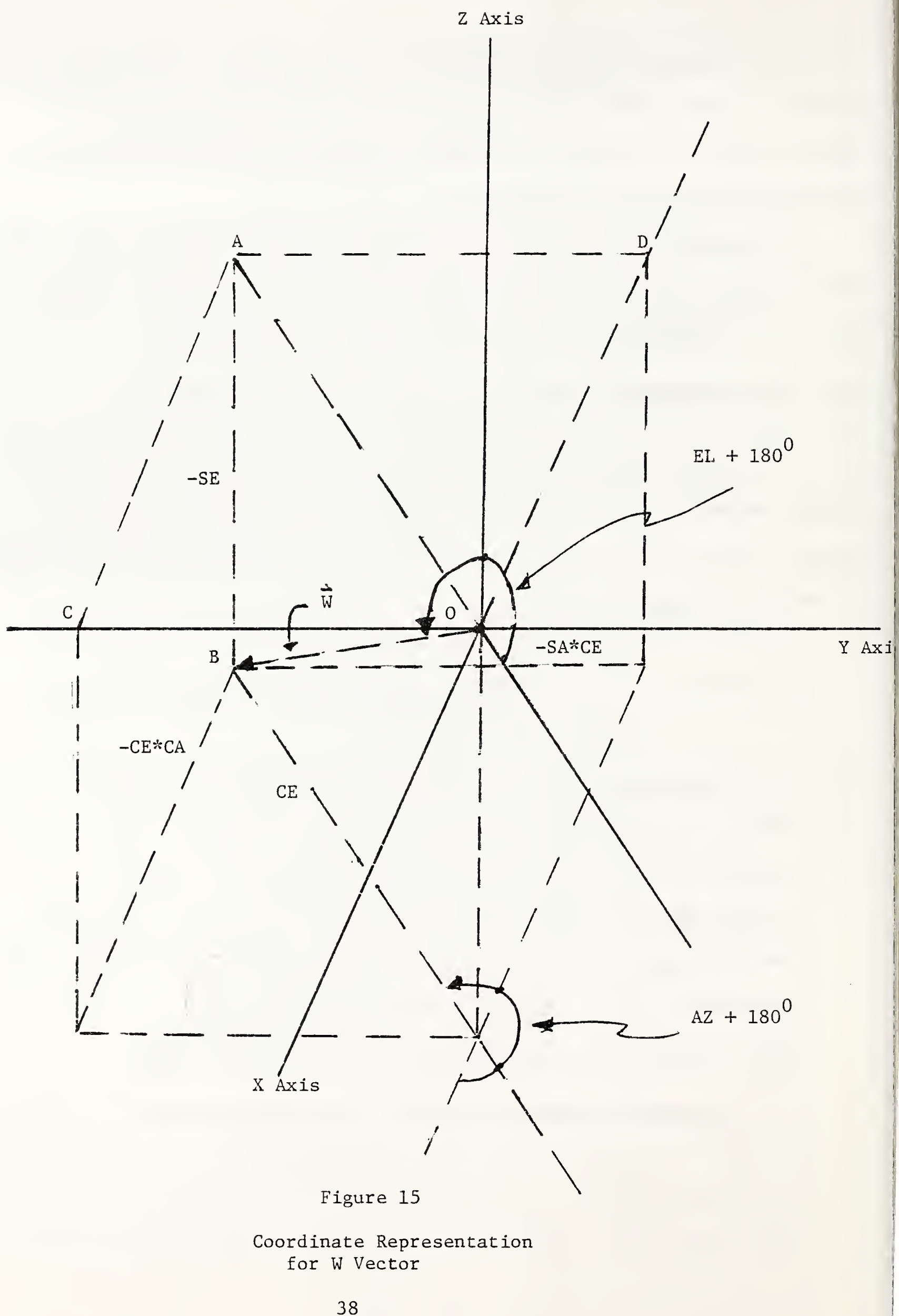




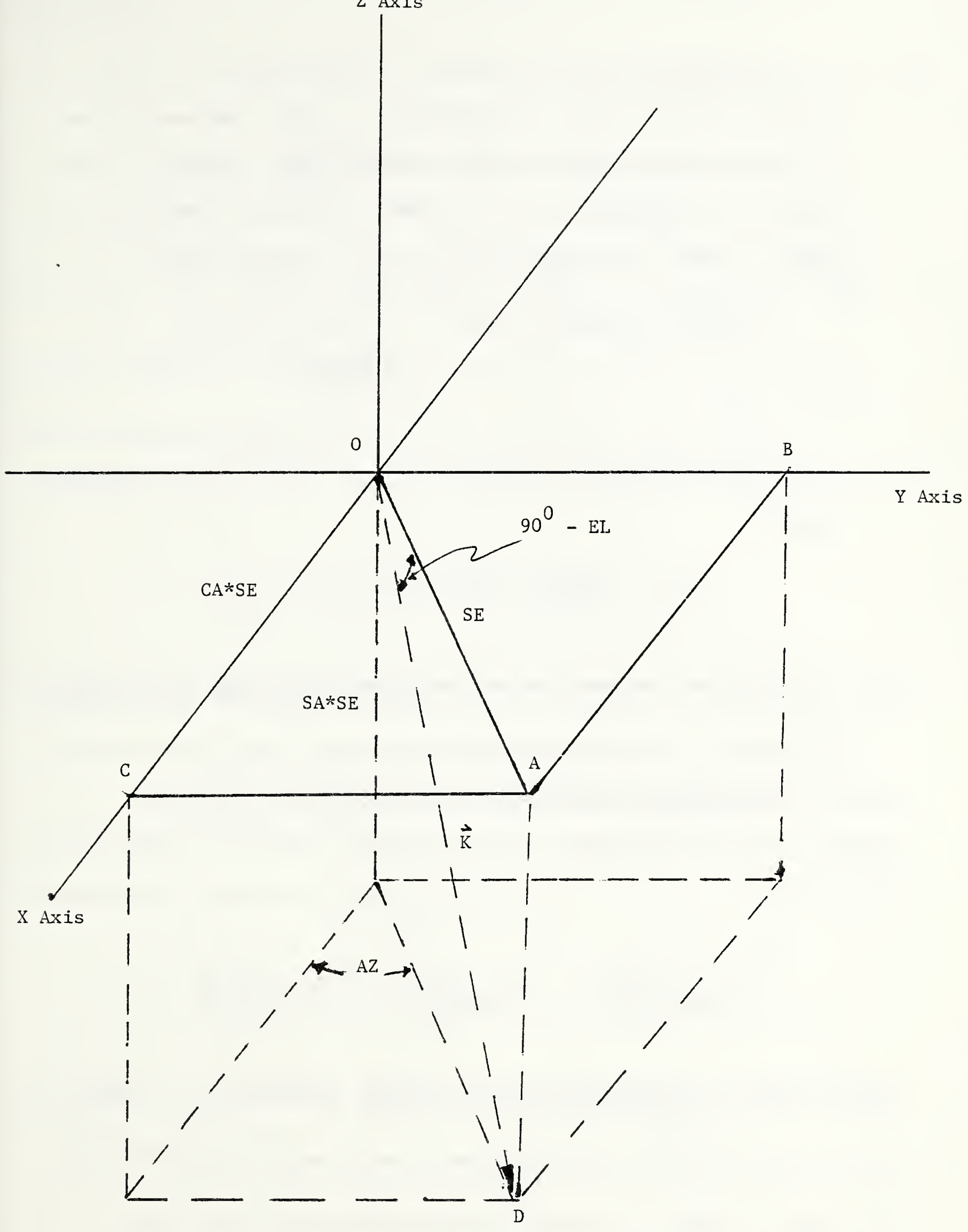

Figure 16

Coordinate Representation

for $\mathrm{K}$ Vector 
2) The development for $\vec{k}$ is similar. See Figure 16 . The distance from $A$ to $D=-\sin \left(90^{\circ}-E L\right)=-C E$. The magnitude of the distance from 0 to $A$ is $\cos \left(90^{\circ}-E L\right)=\sin (E L)=5 E$. Then the $x$ coordinate of $\vec{K}$ is CA*SE and the $Y$ coordinate is SA*SE. Again $\vec{K}$ as specified is a unit vector since

$$
\begin{aligned}
\left.(C A * S E)^{2}+(S A+S E)^{2}+i-C E\right)^{2} & =S E^{2}\left(C A^{2}+S A^{2}\right)+C E^{2} \\
& =S E^{2}+C E^{2} \\
& =1 .
\end{aligned}
$$

3) The definition of the standard cross product of two vectors yields

$$
\vec{k} \times \vec{W}=(-S A, C A, \quad 0)
$$

3.6 The Felation Between Worid Coordinates and Ray Coordinates

Any point in the three-dimensional world coordinate system can be represented uniquely by two orthonormal systems of vectors. The first system is the ordinary system of coordirates given by

$$
\hat{x}=\left(\begin{array}{l}
1 \\
0 \\
0
\end{array}\right), \quad \hat{y}=\left(\begin{array}{l}
0 \\
1 \\
0
\end{array}\right), \quad \ddot{z}=\left(\begin{array}{l}
0 \\
0 \\
1
\end{array}\right)
$$

and the other is given by the orthonormal system $\vec{w}, \vec{k}, \vec{k} \times \vec{w}$. Given a point $\left(\begin{array}{l}x \\ y \\ Z\end{array}\right)$ in the standard coordinate system, then one can write uniquely, as long as the origins are identified, 


$$
\left(\begin{array}{l}
x \\
y \\
z
\end{array}\right)=R \vec{w}+\vec{V} \vec{k}+H(\vec{K} \times \vec{w})
$$

where ( $F, V, H)$ are the coordinates of $\left(\begin{array}{l}X \\ Y \\ z\end{array}\right)$ in the $\vec{W}, \vec{k}, \vec{K} \times, \vec{w}$ system. Given a point $\left(\begin{array}{l}X \\ Y \\ Z\end{array}\right)$ in the standard coordinate system; one can always compute $R, V, H$ by the simple inner product relations

$$
R=\left(\left(\begin{array}{l}
X \\
Y \\
Z
\end{array}\right), \vec{W}\right)=X * W(1)+Y * W(2)+Z * W(3),
$$

implemented in subroutine GETR,

$$
v=\left(\left(\begin{array}{c}
x \\
y \\
z
\end{array}\right), \vec{k}\right)=x * k(1)+y+k(2)+Z * k(3),
$$

implemented in subroutine GETV, and

$$
\left.H=\left(\left(\begin{array}{l}
X \\
Y \\
Z
\end{array}\right), \vec{K} \times \vec{W}\right)=X *((K \times W)(1))+Y *(K \times W)(2)\right)
$$

implemented in subroutine GETH. For the application of $R, V$, and $H$, see Figures $17-20$. 


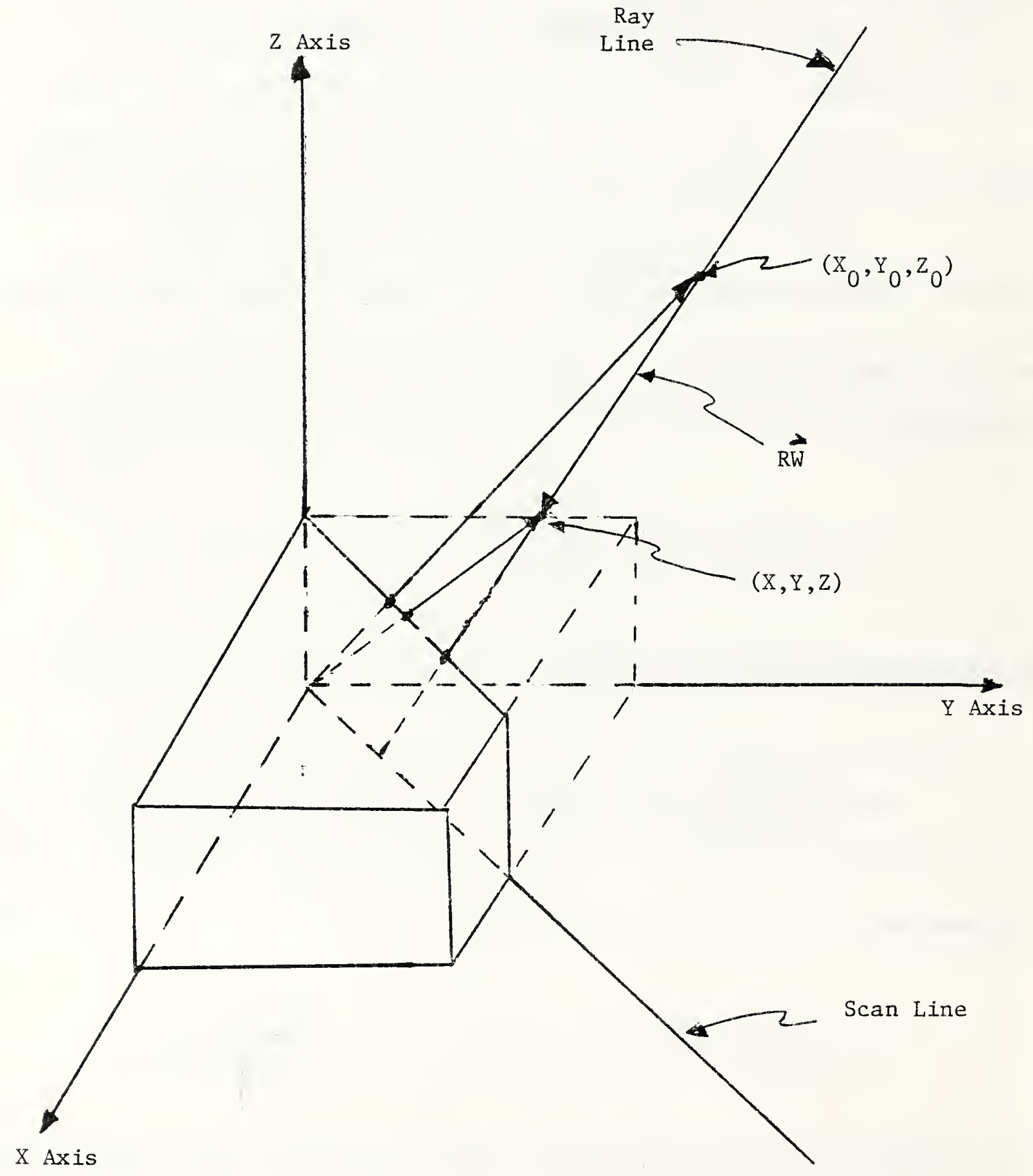

F£gure 17

Indexing Along Rays 


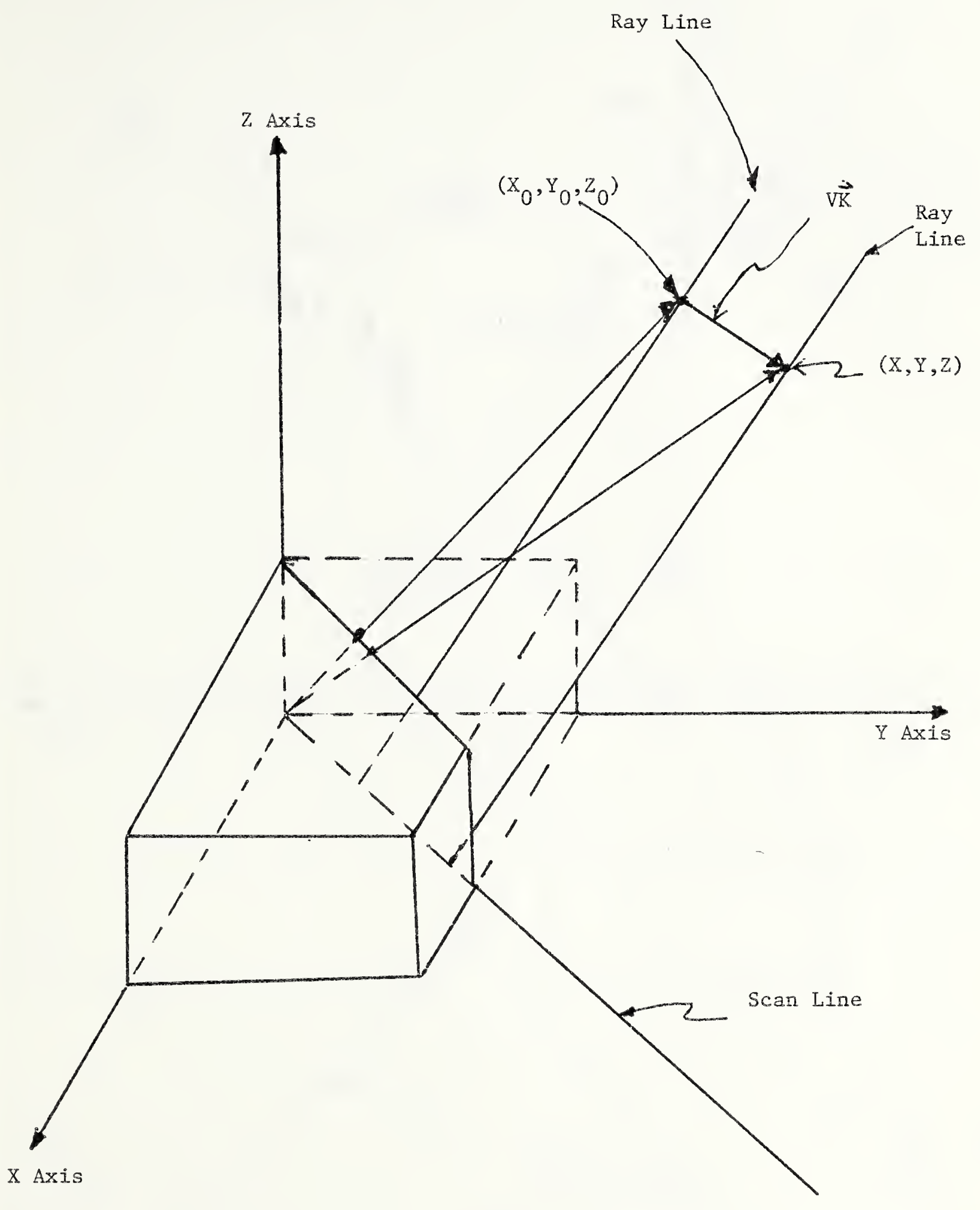

Figure 18

Indexing Different

Rays 


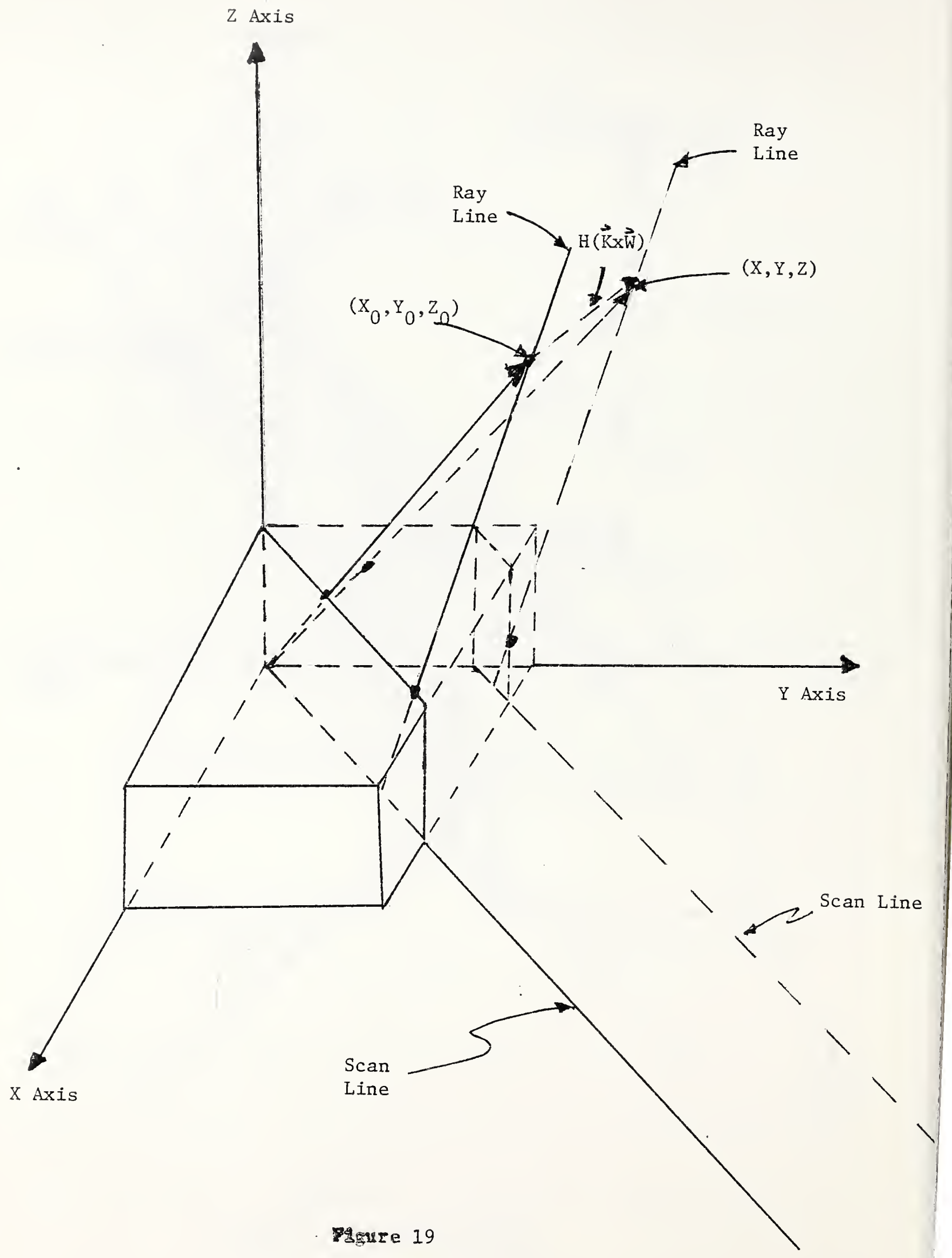

Indexing Different

Ray Planes 


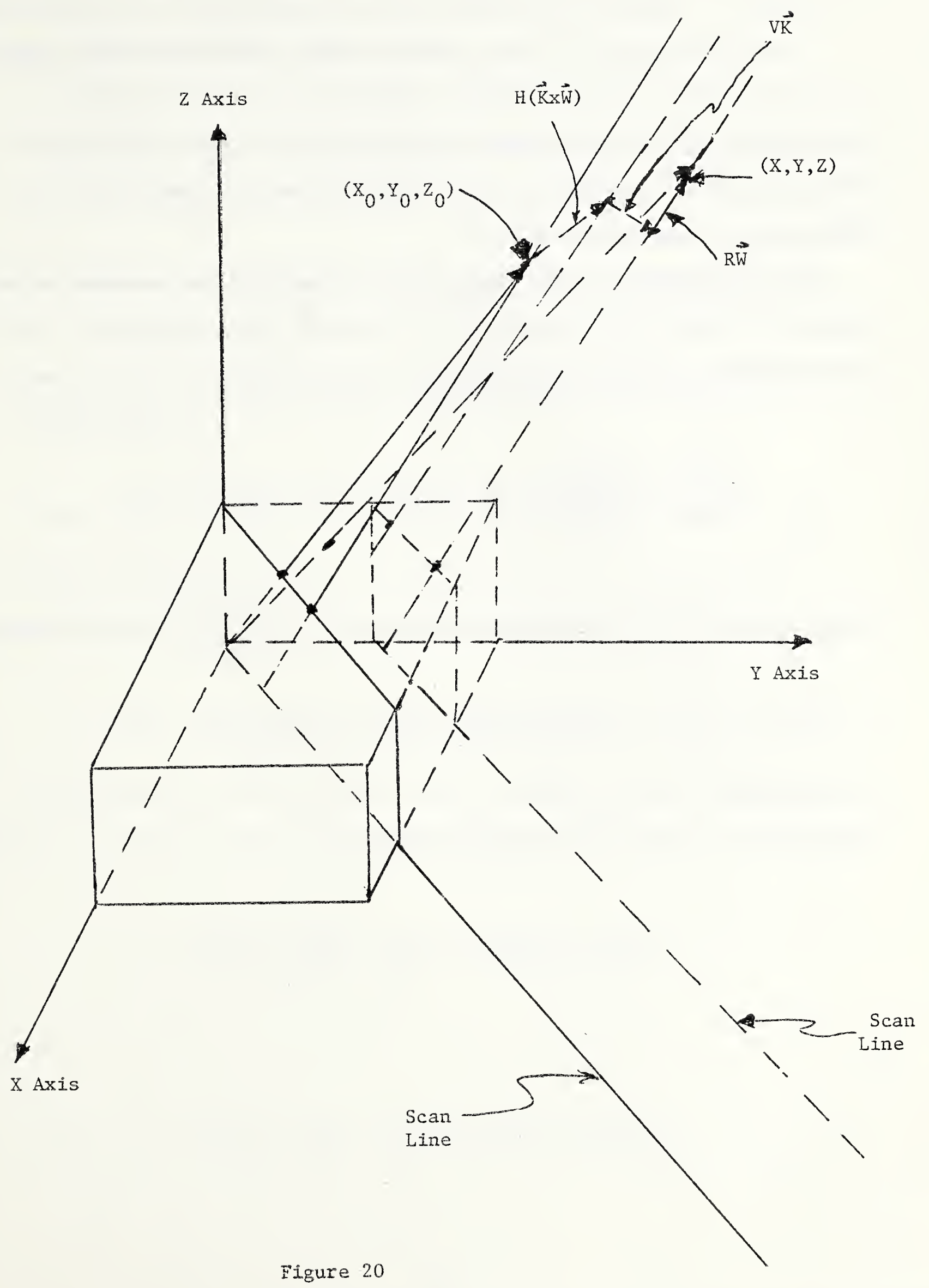

Indexing a Point

from Another 
3.7 Projection of World Foints to the Viewing Window

The coordinates for the viewing space are handled the same as for the light casting space. Foints on a viewplane are addressed by the coordinates $V$ and $H$ since the unit vectors $\vec{K}$, $\vec{k} \ddot{W}$ generate a viewing surface. Points along a viewing ray ara addressed by the coordinate R.

For purposes of simplifying, the viewplane is assumed to be placed so that given a point on the screen $(V, H)$, then a corresponding value in world space, can be found by the formula

$$
\left(\begin{array}{l}
x \\
Y \\
Z
\end{array}\right)=\left(\begin{array}{l}
x_{0} \\
Y_{0}^{0} \\
Z_{0}
\end{array}\right)+\left(V-V_{0}\right) \vec{K}+\left(H-H_{0}\right)(\vec{K} \times \vec{W})
$$

where $V_{0}, H_{0}$ is the viewplane projection of $\left(\begin{array}{l}x_{0} \\ y_{0}^{0}\end{array}\right)$. Conversely, if a point $\left(\begin{array}{l}X \\ Y \\ Z\end{array}\right)$ in world coordinates is specified, then a corresponding row or column in the viewplane can be computed by noting that, since $\vec{K}$ is a unit vector,

$$
\left(v-v_{0}\right) \vec{k} \cdot \vec{k}=\left(\begin{array}{l}
x-x \\
y-Y_{0}^{0} \\
z-z_{0}
\end{array}\right) \cdot \vec{k}
$$

or

$$
v=v_{0}+\left(x-x_{0}\right) \cdot k(1)+\left(y-v_{0}\right) \cdot k(z)+\left(z-z_{0}\right) \cdot k(3) .
$$


This formula is implemented in the subroutine GETfow. A similar argument gets the column as

$$
\begin{aligned}
H= & H_{0}+\left(X-x_{0}\right) \cdot((K \times W)(1))+\left(Y-Y_{0}\right) \cdot(K \times(K)(2)) \\
& \left.+\left(Z-Z_{0}\right) \cdot(K \times W)(S)\right) .
\end{aligned}
$$

This formua is not needed in the program but is given here for the sake of completeness.

3. B Conversion from World Coordinates to Light or Viewing Coordinates

$$
\text { Given a point }\left(\begin{array}{l}
X \\
Y \\
Z
\end{array}\right) \text { in the world coordinates, then it can }
$$

be uniquely represented by $R, V$ and $H$ in the ray coordinates since

$$
\left.\left(\begin{array}{c}
x \\
y \\
z
\end{array}\right)=\overrightarrow{F W}+V \vec{k}+H \vec{k} \times \vec{W}\right)
$$

implies, by taking inner products, that

$$
R=\left(\begin{array}{l}
x \\
Y \\
z
\end{array}\right) \cdot \vec{W}=x(1)+Y W(2)+Z w(3)
$$

$$
V=\left(\begin{array}{l}
x \\
y \\
z
\end{array}\right) \cdot \vec{k}=x k(1)+y K(Z)+Z K(3)
$$




$$
\left.\left.H=\left(\begin{array}{l}
X \\
Y \\
Z
\end{array}\right) \cdot(\vec{K} \times \vec{W})=X(K \times W)(1)\right)+Y(K \times W)(Z)\right) .
$$

These formulas have been implemented in the subroutines GeTf; GETV, EETH, respectivelY.

3.9 Conversion of a Viewplane Point to a uorld Coordinate Foint

Given a point ( $V, H)$ on the viewplane, then

$$
\left(\begin{array}{l}
x \\
y \\
z
\end{array}\right)=\left(\begin{array}{l}
x_{0} \\
y_{0} \\
z_{0}
\end{array}\right)+\left(V-v_{0}\right) \vec{k}+\left(H-H_{0}\right)(\vec{K} \times \vec{H})
$$

associates the $\left(\begin{array}{l}X \\ Y \\ Z\end{array}\right)$ value with that point. This formula is implemented in subroutine GETXYZ.

\$. 10 Computing the Height Along a Fay

Any point has an equivalent representation in the two coordinate systems $\hat{x}, \hat{\gamma}, \hat{z}$ and $\vec{w}, \vec{k}, \vec{k} \times \vec{w}$. This equivalence can be represented by

$$
x \cdot \hat{x}+Y \cdot \hat{y}+Z \cdot \hat{Z}=R \cdot \vec{w}+v \cdot \vec{k}+H \cdot(\vec{k} x \vec{w}) \cdot
$$

Then, given $X, Y$ and $a$ ray index $Y$, one can compute

$$
Z(\hat{Z} \cdot \vec{k})=v-x(\hat{x} \cdot \vec{k})-v(\hat{y} \cdot \vec{k})
$$




$$
z \cdot k(3)=v-x \cdot k(1)-Y \cdot k(2)
$$

and finally

$$
z=(1 / k(3)) \cdot(v-x \cdot k(1)-Y \cdot k(2))
$$

See Table 3.1 
I크므를 포로 1

VECTDR REFRESENTATIONS

$$
\begin{aligned}
& w(1)=\hat{x} \cdot \vec{b}=-C E * C A \\
& k(1)=\hat{x} \cdot \vec{k}=C A * S E \\
& (K \times W)(1)=\ddot{X} \cdot(\vec{K} \times \vec{H})=-S A \\
& W(2)=\hat{Y} \cdot \vec{W}=-C E * S A \\
& K(2)=\hat{Y} \cdot \vec{K}=S A * S E \\
& (K \times W)(2)=\hat{Y} \cdot(\vec{K} \times \vec{W})=C A \\
& W(\vec{B})=\hat{\vec{Z}} \cdot \vec{W}=-5 E \\
& K(\vec{z})=\hat{z} \cdot \vec{k}=-C E \\
& (k \times w)(S)=\hat{z} \cdot(\vec{k} \times \vec{W})=0
\end{aligned}
$$


This section covers the broad details of the mejor algorithms used in this program. The two main algorithms are the shadow graph generation algorithm and the solid projection algorithm. These are supported by two subsidiary algorithms. The first is the entry point selection algorithm which has three components: (1) A case selection look up table, (2) extreme point selection table, and (3) the entry point selection algorithm itself. The second major subsidiary algorithm is the line drawing algorithm. This last algorithm is sometimes referred to in the graphics literature as a scan conversion algorithm.

\section{1 Shadowgraph Algorithm}

This section describes in step form the major tasks performed by the shadowgraph algorithm as it is implemented in the program.

Stege 1: Transfer the data image file from the disk to the first refresh memory of the image processor.

Step 2: Initialize refresh memory 2 of the image processor by blanking it so that the shadowgraph can be created there. This leaves the monitor image all black.

Sttee $3:$ Interactively read in the azimuth and elevation angles for the light source. 
Sttee $4:$ Compute the orthonormal coordinate system $\vec{W}, \vec{K}, \vec{K} \times \vec{W}$ for the 1 ight rays.

Sttee $5:$ From the signs of the $\vec{w}$-vector components, $100 k$ up the current case number.

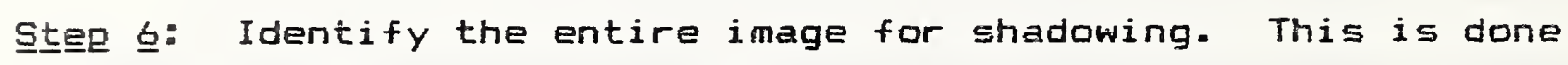
by specifying the picture vertices 35 the refresh memory limits.

StEee $\underline{7}$ : For the current case number given in Step 5, determine the extreme points of the image. See figure 21 for some examples.

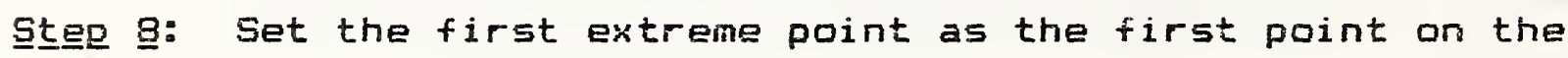
picture plane that a projection of the 1 ight rays onto the plane contacts.

Stee ㅋ: Since this point is not in shadow, transfer its pixel vaiue from refresh memory 1 to refresh memory 2 of the image processor.

Steㄹ 10: For the current case number get the next boundary or entry point of the image in refresh memory 1 at which a projected $\vec{W}$-vector enters the picture. Set this point as $(X, Y)$. If $(X, Y)$ is the second extreme point, go to step 20.

StEeg 11: There is no shadowing at this boundary point, since the light ray encounters this point. Transfer the picture intensity value from refresh memory 1 of the image processor to refresh memory 2 . 
These three sides are not "seen" by rays in the direction $(W(1), W(2))$

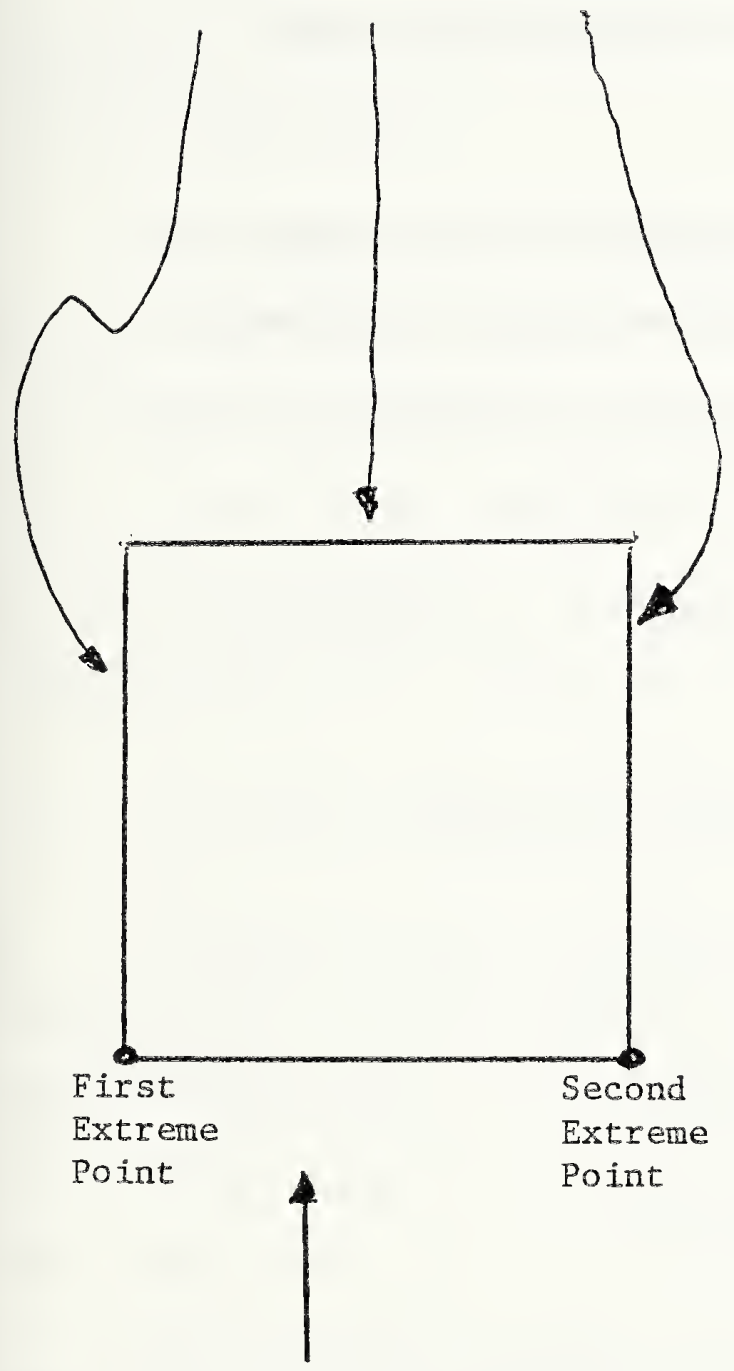

$$
(W(1), W(2))
$$

Example 1
These two sides are not "seen" by the rays in the direction (W(1),W(2))

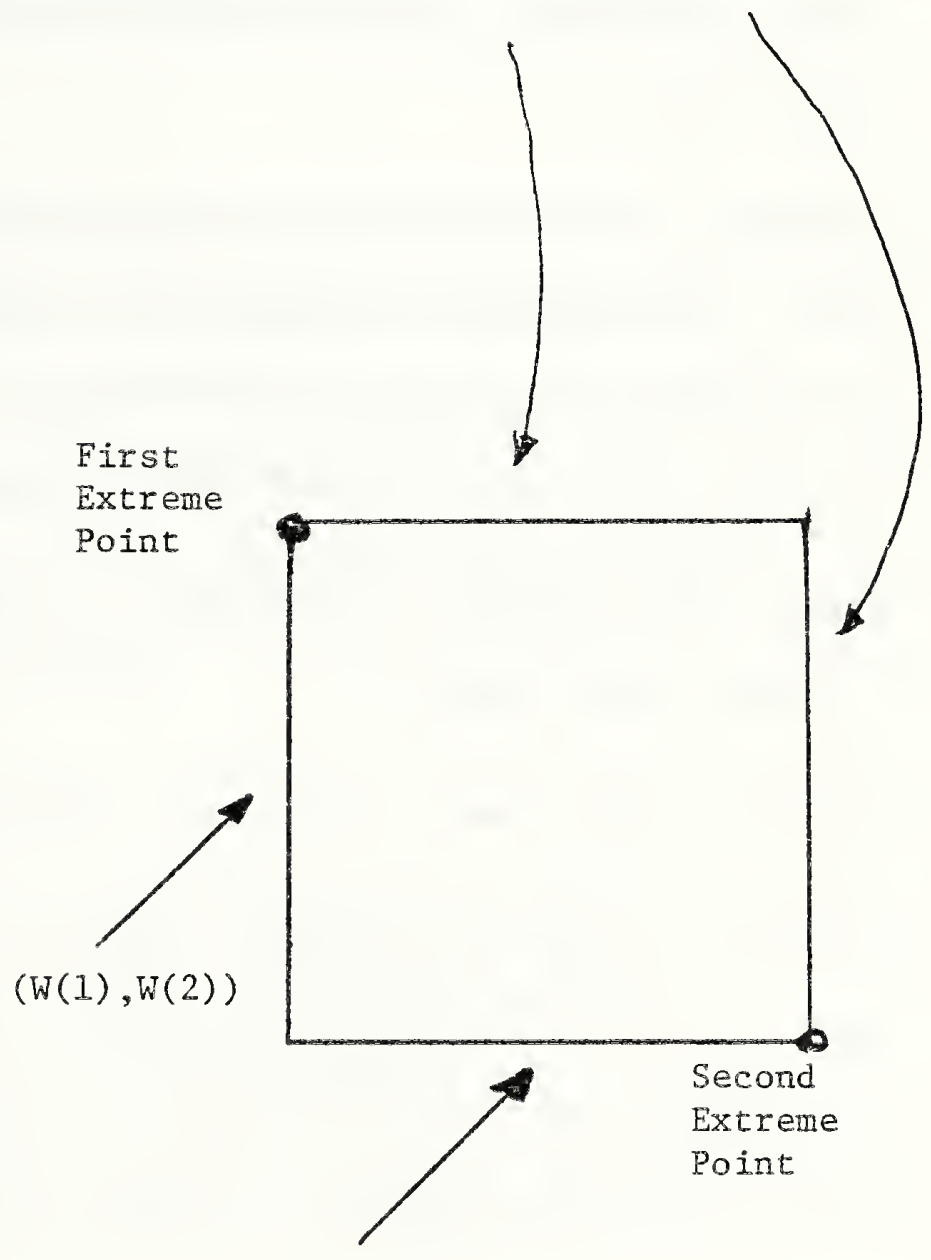

Figure 21

Extreme Point Selection 
Stege 12: Let PICV be the picture value at this boundary point, i.e.s the current $(X, Y)$.

Stㅡㄹ 13: Since there is a unique pIane orthogonal to the XYplane of the image in which the $\vec{W}$-vector 1 ies, compute the unique multiple, $V$, of the $\vec{K}$ vector, in that plane, which identifies a light ray lying in that plane and passing through the point $(X, Y, P I C V)$.

SEteg 14: With the line drawing algorithm generate the next $(X, Y)$ pixel index along the projection of the $\vec{w}-v e c t o r$ on the inage plane. If this point is outside of the picture rectangle, then get the next ray plane by going back to step 10.

SEtee 15: Compute the height of the current ray, indexed by $V$, and call this value $Z T$.

Stege 1ㅇ: Get the image pixel value, PICV, at the point $(X, Y)$.

Steㅡ믈 If If ZT is greater that the image value, PICV, at the point $(X, Y)$, then the pixel is not visible to this ray. Do not write anything at this pixel in refresh memory 2 . Leave the black background there. Go back to step 14.

StEeg 19: If $Z T$ equals the pixel value at the point $(X, Y)$, then write the image value PICV at $(X, Y)$ in refresh memory 1 to the point $(X, Y)$ in refresh memory 2. Since the light ray model assumes that the ray skims the top of a pixel, return to step 14 to generate the next $(X, Y)$. 
Step 19: If $Z T$ is less than the pixel value fICV at the current point $(X, Y)$, then the pixel is seen by the ray. Write the pixel value PICV from refresh memory 1 to refresh memory $Z$ at $(X, Y)$. Get the new index $V$ of the ray that goes through $\left(X_{3}, Y_{3}\right.$ FICV). Return to step 14.

Stee 20: Save the shadowgraph as an indexed file of siz records of 512 bytes each.

An example of a shadowgraph was given previously in figure 5. From the viewer" 5 perspective, both the image and shadowgraph appear as if one were looking vertically downwards at the scene. The orthogonal projection of a world coordinate in the $(X, Y)$ plane translates to the same point on the sereen, but in screen coordinates the $Y$ and $X$ are interchanged.

\subsection{The Solid Projection Algorithm}

Before beginning this algorithm, the image file must be loaded into refresh memory 1 of the image processor and the shadowgraph must also be loaded into refresh memory 2. Furthermore, the contents of refresh memory 2 must be visible on the display monitor. The program steps are as follows:

Step 1: Initialize the cursor and turn it on in order to interactively specify pixel points in refresh menory 2 .

Step 2: Use the trackbal1 cursor to identify two diametrically opposite points of a rectangle of interest in the shadowgraph. This rectangle will be the area converted to a three-dimensional i mage. 
SEtep 푸: Set up the corner vertices so that the upper left is indexed by $(1,1)$. The indexing proceeds counterclockwise from $(1,1)$ to $(2,1)$ to $(2,2)$ to $(1,2)$. See Figure 22 .

Steeㄹ 4 : Identify the center of the rectangle of interest as $(X O, Y O)$ and let $Z O=128$, which is the midpoint of the intensity levels that run from 0 to 255 .

StEeg 5: Draw 1 ines around the rectangle of interest and place a mark at the center. If the viewer does not like this region, return to Step 1 , otherwise continue.

Steㄹ g: Turn off the cursor and initialize a third refresh memory of the image processor for solid projection image.

Steep Z: Interactively get the azimuth and elevation angles for the viewing plane and the percent reduction for shadowing.

Steeg E: Compute the orthonormal vectors for the viewing rays $\vec{W}$, $\vec{k}$ and $\vec{k} \times \vec{w}$.

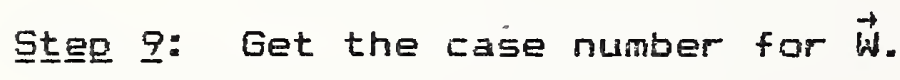

Stee 10: Get the extreme points of the shadowgraph rectangle.

StEeg 11: Set up the first extreme point at the first entry point of the projection of the viewing ray $\vec{W}$-vector onto the XY-plane.

Stene 12: Compute the $H$ multiples of the $\vec{K} x \vec{w}$ unit vector that yield the vertical ray planes passing through the two extreme points. These planes form the left and right bounds for the viewing window. Designate the first as HMIN and the second as HMAX. The 


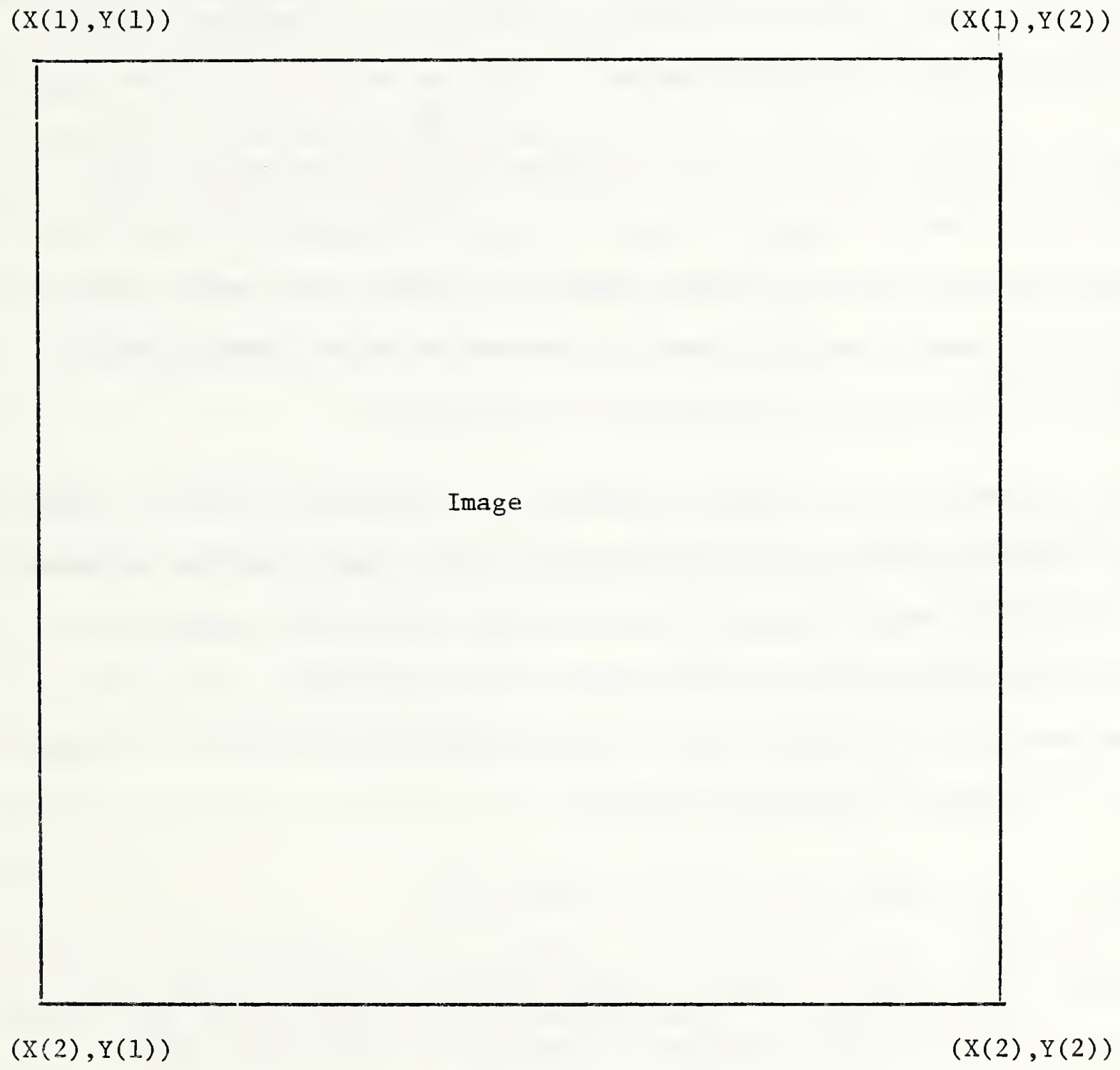

Figure 22

Extreme Point Indexing 
solid is generated by vertical scans on the viewing window moving from left to right after each vertical scan.

Stege 1: Set up the viewport, or monitor screen center, as the projection of the center of the rectangle of interest.

Stege 14: Modify HMIN and HMAX to conform to a viewport that is the smallest to bound the solid.

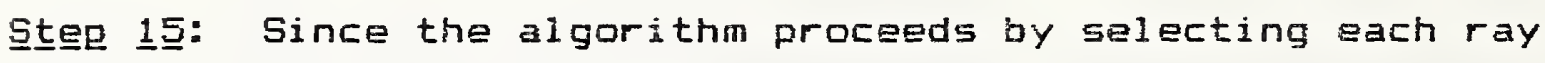
plane from left to right, tracing rays through each pixel from bottom to top in a ray plane, begin by setting $H=H M I N$. Get the starting viewport column for this $H$ and set the starting row as 511 which represents the bottom of the screen.

Sten 19: On the first pass through this step, the first extreme point is designated as the beginning entry point to the rectangle of interest, but the algorithm picks the next entry point to begin. If the second extrame point is encountered, stop the algorithm and go to Step 27. Set the entry point as the current world cocrdinate point of interest.

Steep 17: Increment the column counter by 1 .

StEg 1요 Eet the row index on the viewing window of the entry point $\{X, Y, O\}$ in the $X Y-p l$ ane of the world coordinate space.

Stęep 19: Once the row and column indices have been selected on the viewing plane, specify this as the current screen point. 
Steg 20: Get the corresponding world coordinate point for the current screen point, i.e. row and column, on the viewplane. Note that this is not the same world coordinate point as $(X, Y, O)$.

Step 21: Get the ray index $V$ of the ray through this viewplane world coordinate point.

Step 22: Get the $Z$ value on the ray indexed by $Y$ at the current

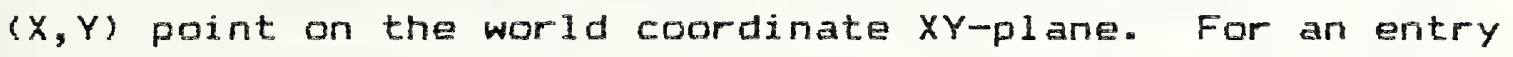
point, this $z$ value will be 0 .

Step 23: Get the pixel value from the image in refresh memory 1 and the shadowgraph value from refresh memory 2 for the ( $X, Y$ ) point.

Steg 24: If the ray haight $Z T$ is greater than the image value FICV at the current $(X, Y)$ point on the plane, then the ray does not see the pixel. Generate the next $(X, Y)$ point along the ray projection on the XY-plane. Test whether it remains within the rectangle of interest. If it does, go back to step 22, otherwise go back to step 16 to move to the next ray plane or sereen column.

Step 25: If the height $2 T$ is equal to the pixel value, the pixel is seen. The current ray is not continued. A new ray is generated through the next screen point above it and tracing continues. This is done by first writing the pixel value from the original image in refresh memory 1 to the viewport refresh menory, i.e. refresh memory 3 , at the projected $(V, H)$ coordinate. Feduce the pixel value by the pereent required for shadowing if 
the intensity value on the shadowgraph in refresh memory 2 is 0 at that pixel. Move up one pixel in the viewport column and get the associated world coordinate $(X, Y, Z)$ for this point on the viewplane. Get the $V$ index for the ray through this point. Generate the next $(X, Y)$ point along the projected $\vec{w}$ vector 1 ine on the $(X, Y)$ plane. Go back to Step 22.

Stㅡeᄅ 2으: If ZT is less than the pixel value at the current $(X, Y)$, then the pixel is seen by the ray. If the intensity of the associatad pixel in refresh memory 2 is 0 , then this indicates that the point is in shadow. Write out to refresh memory 3 the height $Z T$ reduced, if necessary, by the percent specified if shadowing is indicated. Decrement the row index to move up one row. Get the $(X, Y, Z)$ world coordinate that is equivalent to the new viewplane point. Get the $V$ index for the ray through this point. Go back to step 22.

StEe 27 : Write out the solid image to the disk if desired.

\subsection{Case Selection Table}

Table 4.1 specifies a case index that can be referenced by ather subroutines in the program. It distinguishes each passible case combination of the first two components of the $\vec{W}$-vector that points along the rays from either the light source or viewer towards the origin.

\section{4 Extreme Point Selection Table}

Depending on the direction vector $(w(1), W(2))$ along the base plane of the solid, this table specifies the first and the 
I므믈 4.1

\section{$\vec{w}$-VECTOR CASES}

\section{Case}

IngEx

$w(1)=0, w(2)=0$
$w(1)=0, w(2)>0$
$w(1)=0, w(2)<0$
$w(1)>0, w(2)=0$
$w(1)>0, w(2)>0$
$w(1)>0, w(2)<0$
$w(1)<0, w(2)=0$
$w(1)<0, w(2)>0$
$w(1)<0, w(2)<0$


포므므트 노를

EXTFEME FOINT TABLE

\section{트로으}

1. $W(1)=0, W(2)=0$

2. $W(1)=0, W(2)>0$

3. $w(1)=0, w(z)<0$

4. $W(1)>0, W(2)=0$

5. $w(1)>0, w(2)>0$

b. $w(1)>0, w(2)<0$

7. $w(1)<0, w(2)=0$

B. $W(1)<0, W(2)>0$

9. $W(1)<0, W(2)<0$
Extrenge

Eoi몬 1

\section{Extrenge \\ Point 2}

Fiag Returned

$(X(1), Y(1))$

$(X(2), Y(1))$

$(X(2), Y(Z))$

$(X(1), Y(2))$

$(X(1), Y(2))$

$(X(1), Y(1))$

$(X(1), Y(2))$

$(X(2), Y(1))$

$(X(2), Y(Z))$

$(X(1), Y(1))$

$(X(2), Y(1))$

$(X(2), Y(2))$

$(X(1), Y(1))$

$(X(2), Y(2))$

$(X(2), Y(1))$

$(X(1), Y(2))$ 
last base point of the boundary rectangie encountered by the rays. See Figure 21 for an illustration.

Let the four vertices be 1 abeled with $X$ and $Y$ components as shown in Figure 22: $(X(1), Y(1))$ is the upper left corner, $(X(2), Y(1))$ is the lower left corner, $(X(1), Y(2))$ is the upper right corner and $(X(2), Y(2))$ is the lower right corner. For each case there are two extreme points. These are detailed in Table 4.2.

\subsection{Entry Foint Algorithm}

By an entry point is meant a point on the boundary of a base rectangle through which the projection of a ray in space onto the XY-plane passes as it traverses across the base rectangle. See Figure 4 for an illustration. This algorithm begins with the assumption that there is a current entry point. The algorithm returns the next entry paint or a fiag if an extreme point is encountered. Let IXIN, IYIN De the current entry point. The algorithm is a case-by-case anaiysis.

C토토 1: $W(1)=w(z)=0$. Feturn a flag.

드으 2: $W(1)=0, W(2)>0$. Aeginning with extreme point $(X(1), Y(1))$, set $I X I N=X(1)$, IYIN $=Y(1)$. The new gntry point is then defined by IXIN = IXIN+1, IYIN = IYIN. This case terminates when IXIN-X(Z)=0 and IYIN-Y $(1)=0$.

Case $5: W(1)=0$. W(2) $\quad$ W. Beginning with the first extreme point IXIN $=X(2)$, IYIN $=Y(2)$, set the next entry point as $I X I N=I X I N-1$, IYIN $=$ IYIN and stop when $I X I N-X(1)=I Y I N-Y(2)=0$. 
드트르 4: W(1) $>0, W(2)=0$. Begin with IXIN $=X(1)$, IYIN $=$ $Y(2)$. Set the next entry point as IXIN = IXIN, IYIN = IYIN-1. Stop when IXIN-X(1) = IYIN-Y(1) = 0.

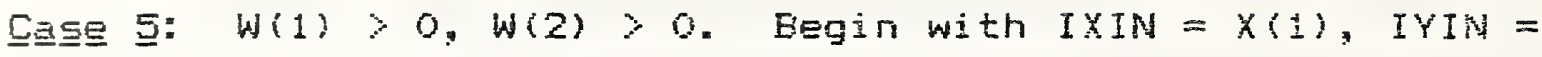
$Y(2)$. Set the next entry point as IXIN = IXIN, IYIY = IYIN-1 until IXIN-X(1)=IYIN-Y(1)=0. Then set the next entry point aSIXIN = IXIN+1, IYIN = IYIN. Stop when IXIN-X(Z)=IYIN-Y(1)= O.

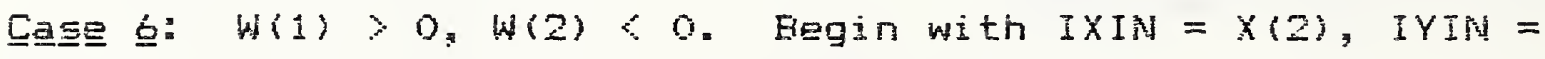
Y(2). Set the next entry point to IXIN = IXIN-1, IYIN = IYIN urtil IXIN-X(1) = IYIN-Y(Z) = 0 . Then set the next entry point to IXIN =IXIN, IYIN = IYIN-1. Stop when IXIN-X(1)=IYIN-Y(1)= 0.

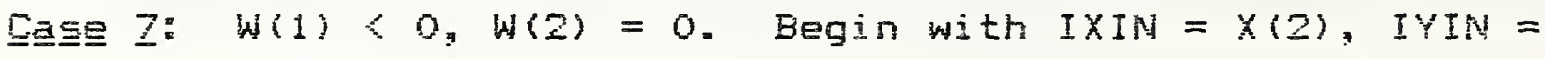
Y(1). Set the next entry point to IXIN - IXIN, IYIN - IYIN+1. Stop when IXIN-X(Z)= IYIN-Y(Z) $=0$.

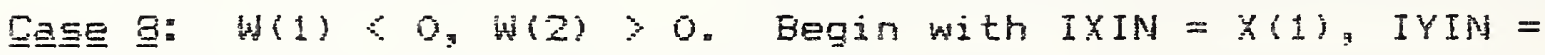
Y(1). Set the next entry point IXIN = IXIN+1; IYIN - IYIN untiI IXIN-X(Z) = IYIN-Y(1) = D. Then set the next entry point to IXIN $=I X I N, I Y I N=I Y I N+1$. Stop when $I X I N-X\{Z)=I Y I N-Y(Z)=0$.

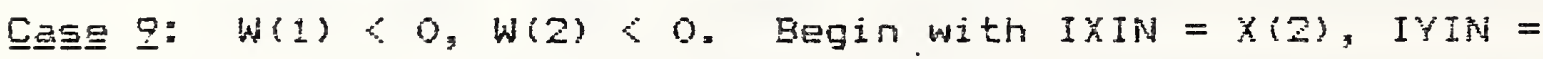
Y(1). Set the next entry point to IXIN = IXIN, IYIN - IYIN+1 untiI IXIN-X(2)=IYIN-Y(Z)=0. Then set the next entry point to $I X I N=I X I N-1$, IYIN $=$ IYIN. Stop when IXIN-X(1)=IYIN-Y(2) $=0$ 
4.6 Line Drawing Algorithm

The task of a line drawing algorithm is to compute the coordinates of the pixels that lie near a line on a twodimensional raster grid in such a manner that when the pixels are strung together, they approximate the straight Iine isee Figure 22). There are several such algorithms in the literature and they are sometimes peferped to as scan-conversion algorithms. Ordinarily, the algorithms are applied to the problem in which two endpoints of the line are specified. In the present case; an algorithm will be presented in which the starting value and the direction vector of the line are given. The problem then is to start from a point on the line and generate the next pixel along the Iine. The pixels chosen are based on integer truncation rather than rounding.

Assume that a point $(X, Y)$ is given and let (IX,IY) be the point composed of the integer truncated values af $X$ and $Y$. This point will be referred to as the current pixel. Furthermore, suppose that a direction vector in the XY-piane has been given by $(W(1), W(2))$.

므롤 1: $W(1)=W(2)=0$

RETUFN Error $f l a g$.

Case 2: $W(1)=0, w(2)>0$

$$
\begin{aligned}
& \text { IF } Y>=0 \text {, FETURN }(I X, I Y+1) \text {. } \\
& \text { IF } Y<0 \\
& \text { AND IF IY }>Y, \text { RETURN }(I X, I Y)
\end{aligned}
$$

DTHERWISE IF IY $Y Y$, FETURN $(I X, I Y+1)$. 
드르르 표 $\quad W(1)=0, \quad W\{2\}<0$

IF $Y y=0$

AND IF IY $Y, \quad$ RETLIFN $(I X, I Y):$

DTHERWISE IF $I Y=Y$, FETUFIN $\{I X, I Y-1\}$.

IF $Y<0$, RETUFN $(I X, I Y-1)$.

드토르 4: $(1)>0, \quad$, $(2)=0$

IF $x y=0$, FETLFN $(I X+1, I Y)=$

If $x<0$

AND IF IX $Y X$, FETUFH (IX,IY)?

OTHERUISE IF IX $=X_{3}$ FETUFN $(I X+1, I Y)$ :

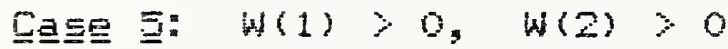

LET SLOFE $=W(2), H(1)$.

IF $X y=0$, LET $X T=I X+1$.

IF $x<0$

AND IF IX $Y, \quad$ LET $X T=I X ;$

DTHEFWISE IF IX $I X$, LET $X T=I X+1$.

LET YT = SLDFE * $(X T-X)+Y$.

IF $Y\rangle=0$ AND IY $Y Y T=\zeta I Y+i$

RETUFA (IXT, IYT)

WHERE IXT = INTEGER TRUNCATED XT

IYT = INTEEER TFUNCATED YT.

IF $Y=0$ AND IY+1 YT.

LET $Y T=I Y+1$

LET $X T=(1 . /$ SLOFE $) *(Y T-Y)+X_{9}$

TRLNCATE XT TO IXT:

TRUNICATE YT TO IYT:

RETUFN (IXT, IYT). 


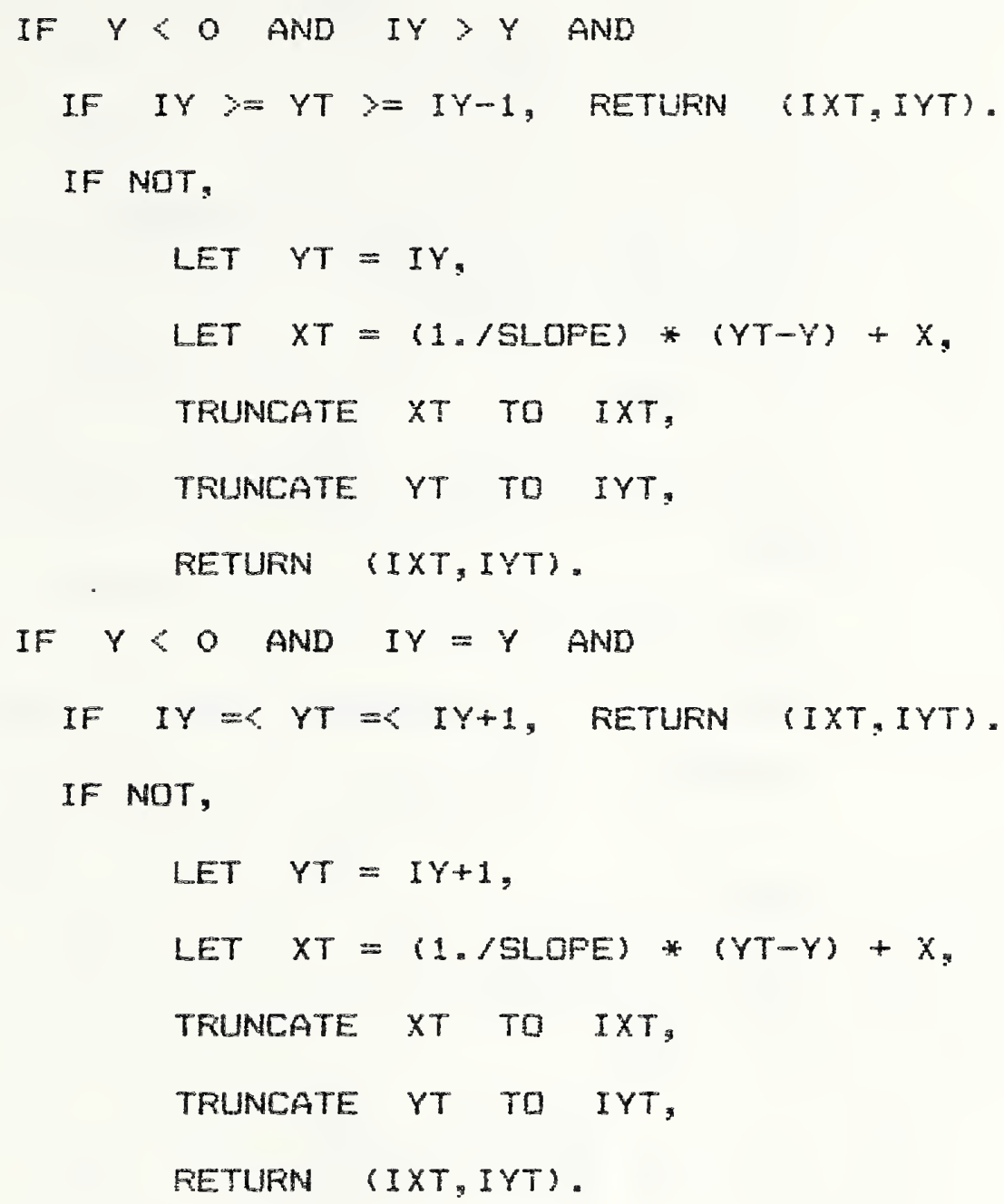

Ca르 $6: W(1)>0, W(2)<0$

LET SLOPE $=W(2) / W(1)$.

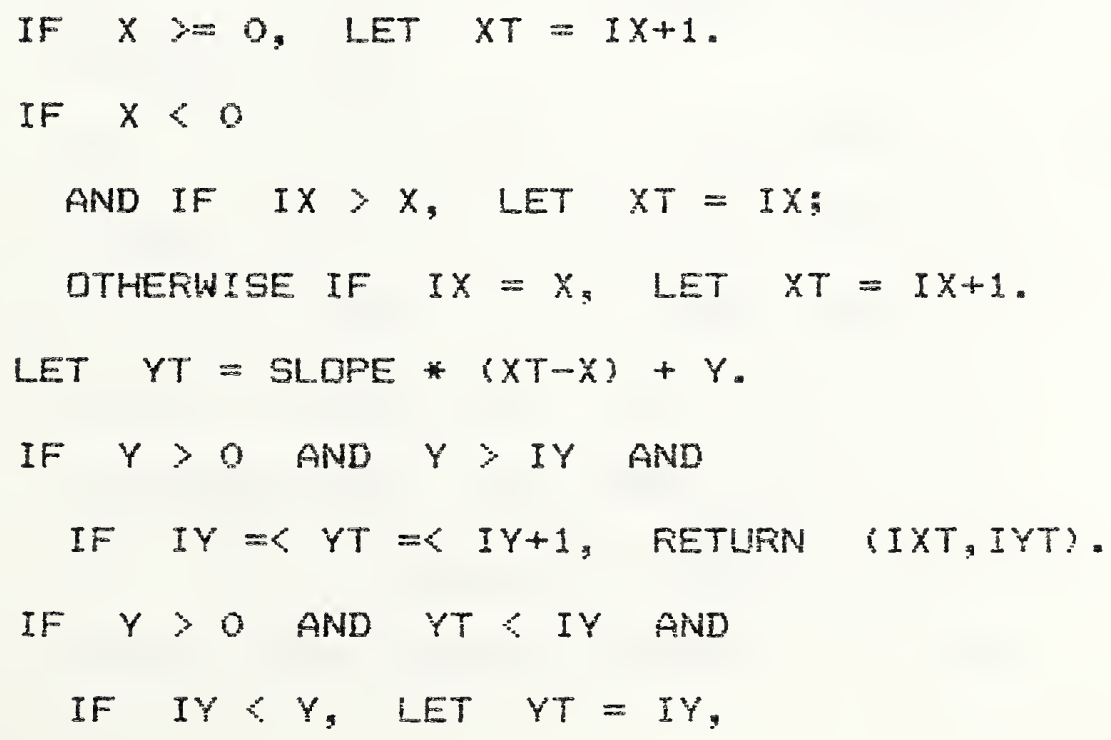


LET $X T=(1 . / S L O F E) *(Y T-Y)+X$, FETURN (IXT,IYT).

IF IY $=Y$, LET $Y T=I Y-1$,

LET $X T=(1 . /$ SLOFE $) *(Y T-Y)+X$, RETURN (IXT,IYT).

IF $Y<O$ AND

IF IY $\rangle=Y T\rangle=I Y-1$, FETURN (IXT,IYT).

IF NOT,

LET $Y T=I Y-1$

LET $X T=(1 . / S L O F E) *(Y T-Y)+X g$

FETURIN (IXT, IYT).

므토 Z: $W(1)<0, W(2)=0$

LET SLOPE $=W(2) / W(1)=0$.

IF $x>=0$ AND

IF $X>I X$, LET XT - IX:

IF $X=I X$, LET $X T=I X-1$.

IF $X<0$, LET $X T=I X-1$.

LET YT $=$ SLOFE * $(X T-X)+\gamma$,

RETUFN (IXT,IYT).

므트르 8: $W(1)<0, W(2)>0$

$$
\begin{aligned}
& \text { LET } \text { SLQFE }=W(2) / W(1) . \\
& \text { IF } X>=0 \text { AND } \\
& \text { IF } X>I X, \text { LET } X T=I X ; \\
& \quad \text { IF } X=I X, \text { LET } X T=I X-1 . \\
& \text { IF } X<O, \text { LET } X T=I X-1 . \\
& \text { LET } Y T=\text { SLOFE } *(X T-X)+Y .
\end{aligned}
$$




$$
\begin{aligned}
& \text { IF } Y>=0 \text { AND } \\
& \text { IF } \quad \text { IY }=\langle Y T=\zeta \quad I Y+1 \text {, } \\
& \text { RETURN (IXT,IYT); } \\
& \text { DTHERWISE IF IY } Y \text {, LET YT }=I Y \text {. } \\
& \text { LET } X T=(1 . / \text { LLPEE } *(Y T-Y)+X \text {, } \\
& \text { RETUFN (IXT, IYT). } \\
& \text { IF } Y<0, \quad \text { LET } Y T=I Y-1 . \\
& \text { LET } X T=(1 . / S L D F E) *(Y T-Y)+X, \\
& \text { RETURN (IXT, IYT). }
\end{aligned}
$$

드롤 ㅇ: $W(1)<0, W(2)<0$

$$
\begin{aligned}
& \text { LET SLOPE }=W(2) / W(1) . \\
& \text { IF } x y=0 \text { AND } \\
& \text { IF } X>I X, \text { LET } X T=I X . \\
& \text { IF } X=I X, \text { LET } X T=I X-1 . \\
& \text { IF } X \subseteq 0, \text { LET } X T=I X-1 ! \\
& \text { LET } Y T=\text { SLOPE * }(X T-X)+Y \text {. } \\
& \text { IF } Y Y=0 \text { AND } \\
& \text { IF IY } Y Y \text { AND IY } \angle=Y T<=I Y+1 \text {, } \\
& \text { RETUFN (IXT, IYT). } \\
& \text { DTHEFWISE LET YT = IY, } \\
& \text { LET } X T=(1 . / S L Q P E) *(Y T-Y)+X, \\
& \text { FETURN (IXT, IYT). } \\
& \text { IF IY }=Y \text { AND IY } y=Y T y=I Y-1 \text {, } \\
& \text { RETURN (IXT, IYT). } \\
& \text { OTHEFINISE LET YT }=\text { IY-1, } \\
& \text { LET } X T=(1 . / \text { SLOFE }) *(Y T-Y)+X, \\
& \text { RETURN (IXT, IYT). }
\end{aligned}
$$




$$
\begin{aligned}
& \text { IF } Y<0 \text { AND } \\
& \text { IF IY } Y=Y T y=I Y-1, \\
& \text { RETURIN (IXT, IYT). } \\
& \text { OTHERWISE LET YT=IY-1, } \\
& \text { LET } X T=(1 . / S L O F E) *(Y T-Y)+X, \\
& \text { RETURN (IXT,IYT). }
\end{aligned}
$$





\section{1 System Commands}

When the user types solid on the console to begin the program, the host system transfers to the foilowing file:

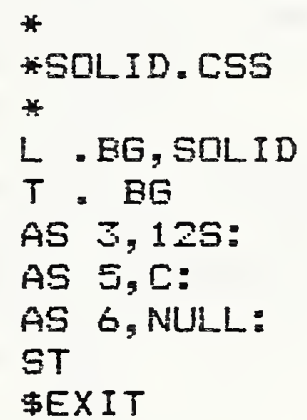

The first three i ines are comments identifying this command file as SOLID.CSS. The fourth line loads the 1 inked task with the name SOLID and gives it the system designated name. BG for a background job if the multiterminal environment is not active. If the multiterminal environment is active, the system identifies the job with the user name entered at sign-on time. The next line identifies any following assignments with the task just loaded. The next three 1 ines assign logical unit number 3 to the image processor, known to the operating system by the mremonic I2S:, logical unit 5 to the user"s terminal and logical unit b to a null device. This means that the logical unit o is assigned to the task, but any input/output through it will be ignored. This is inserted so that the user could assign logical unit 6 to an input/output unit for progran error anaiysis at a later time, if necessary. The next to the last 1 ine starts the designated task and the final I ine exits to the user console at progr termination. 


\subsection{Main Frogran}

\subsubsection{Summary}

This subsection contains the flow chart and 1 isting of the main program. It implements both the shadowgraph algorithm and solid generation algorithm. The user selects which algorithm to use interactively.

In the shadowgraph algorithm, the program traces individual rays from the light source to the surface. The light source is located at a sperified azimuth and elevation angle, selected interactively by the user. As each ray is traced to the surface, the height along the ray is either greater thang equal to, or less than a pixel height ropresenting a topographic amplitude. If the ray height is greater than the pixel value, that pixel is not seen by the ray and falls into shadow relative to tine ray. If the ray height is equal to the pixel height, then the pixel is seen and the ray is continued as well. If the ray has a height less than the pixel, the pixel is seen and a now ray is selected that touches the tip of the pixel. Tracing then continues along the new ray.

In the solid generation algorithm, rays are traced from the viewing plane to the surface. If a pixel is seen, then the height of the ray at contact is projected back to the viewing Plane, modified by an intensity reduction factor if the pixel lies in shadow. If a pixel is not seen, then the ray is gontinued. 


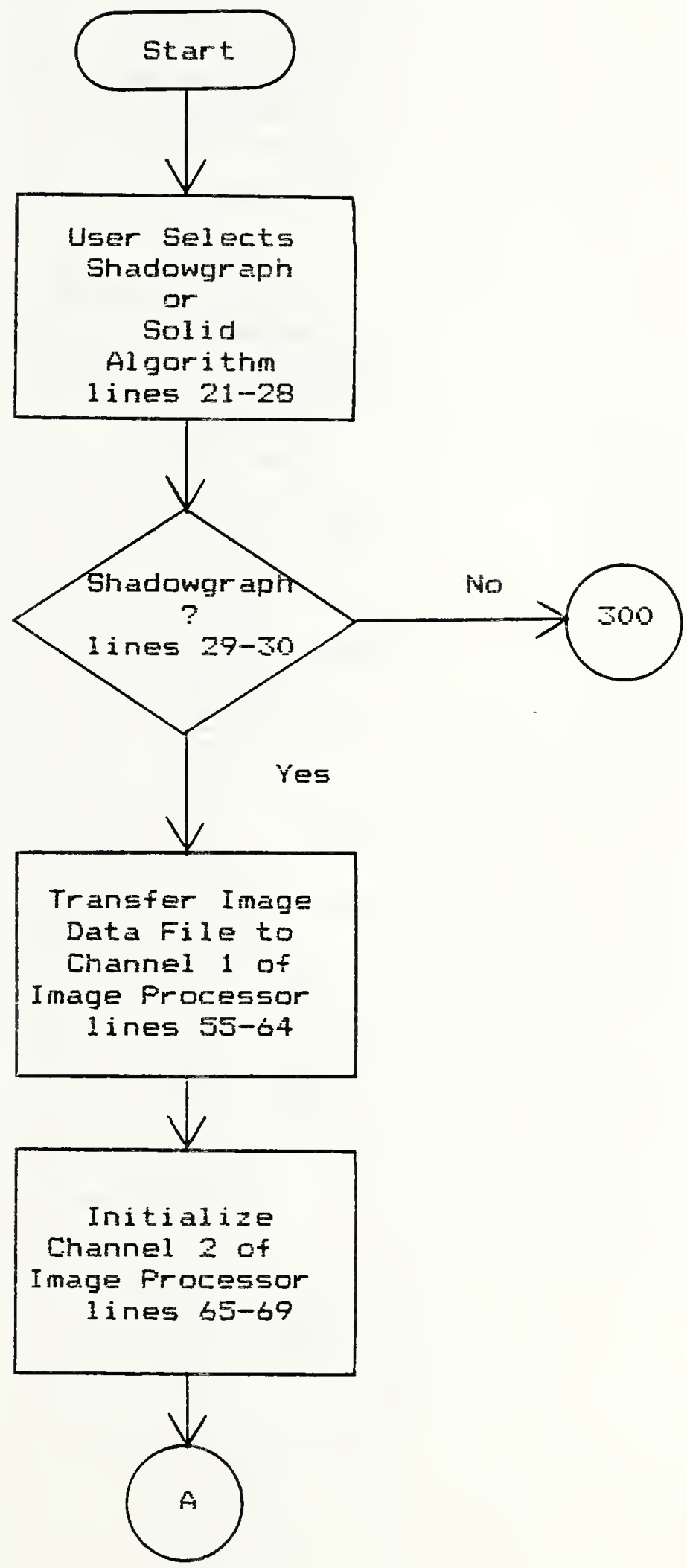



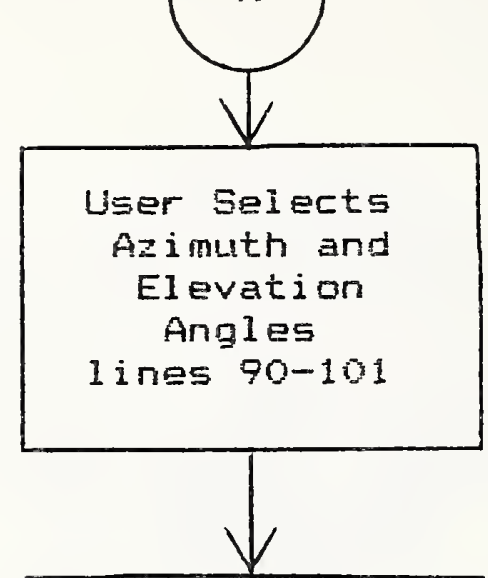

Compute

Conversion Factor

From Angles

to Fiadius

1 ines 102-103

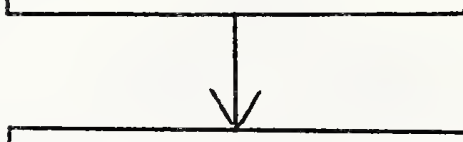

Compute Cosines and Sines of

the Azimuith and

Elevation

lines $110-115$

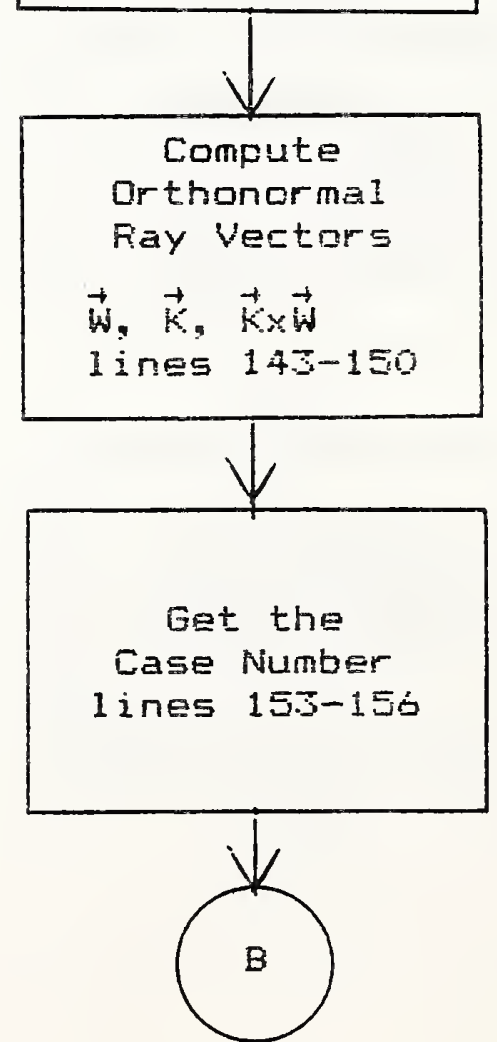




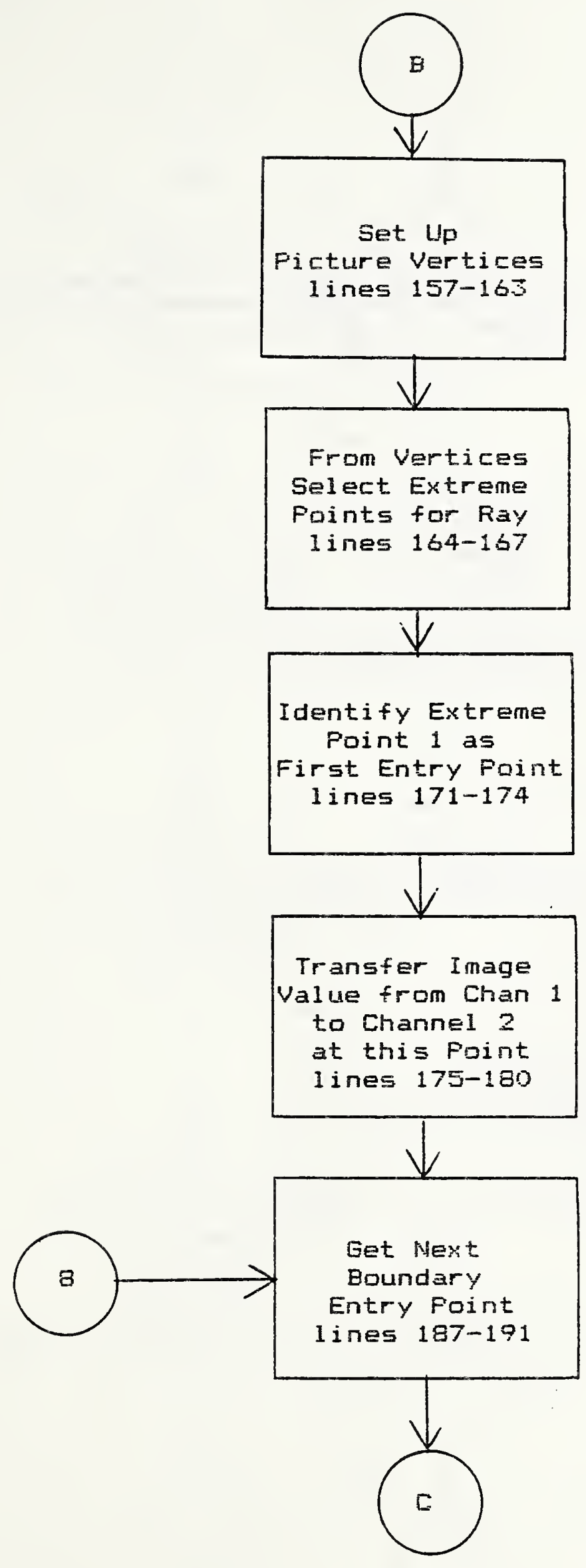




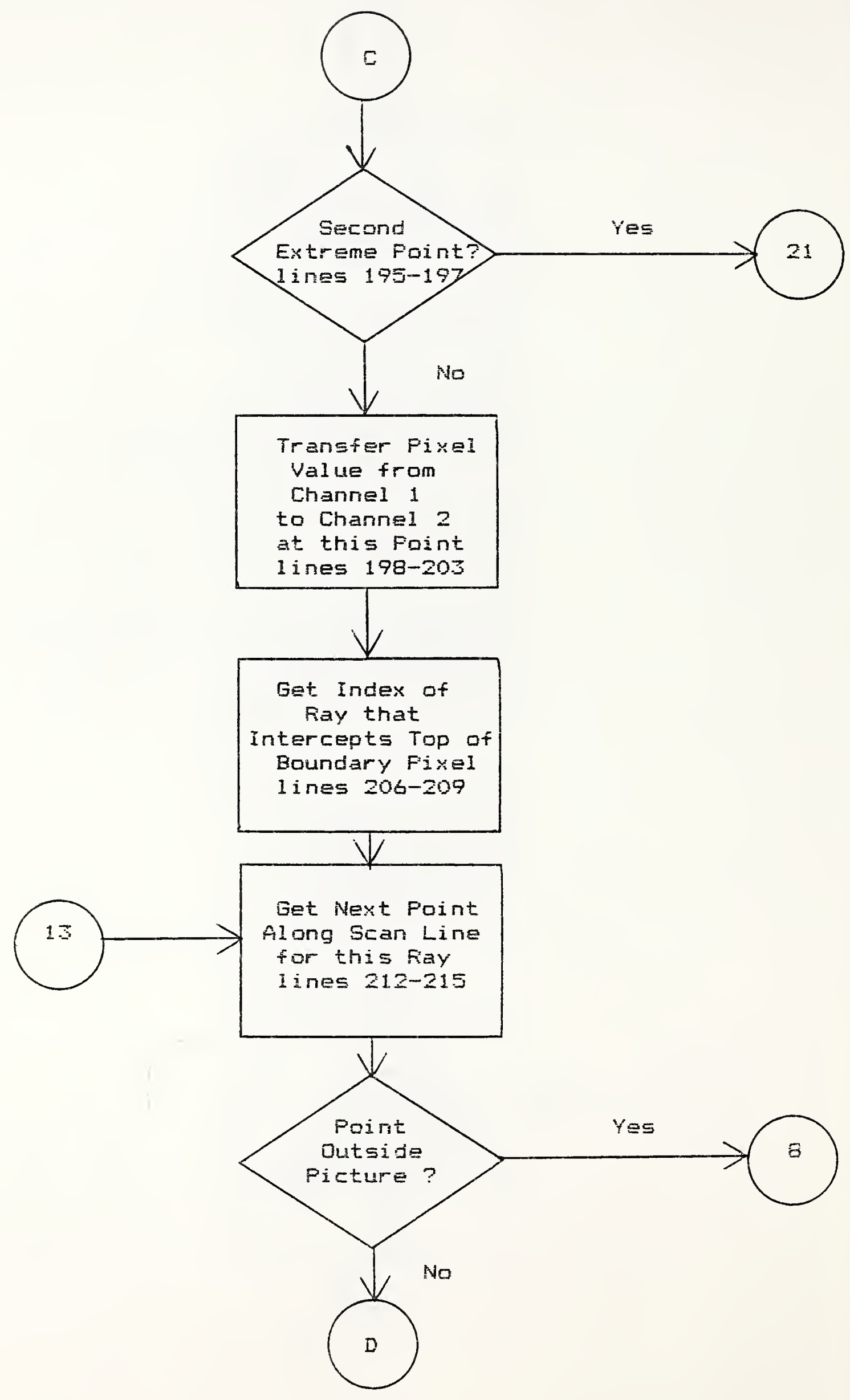




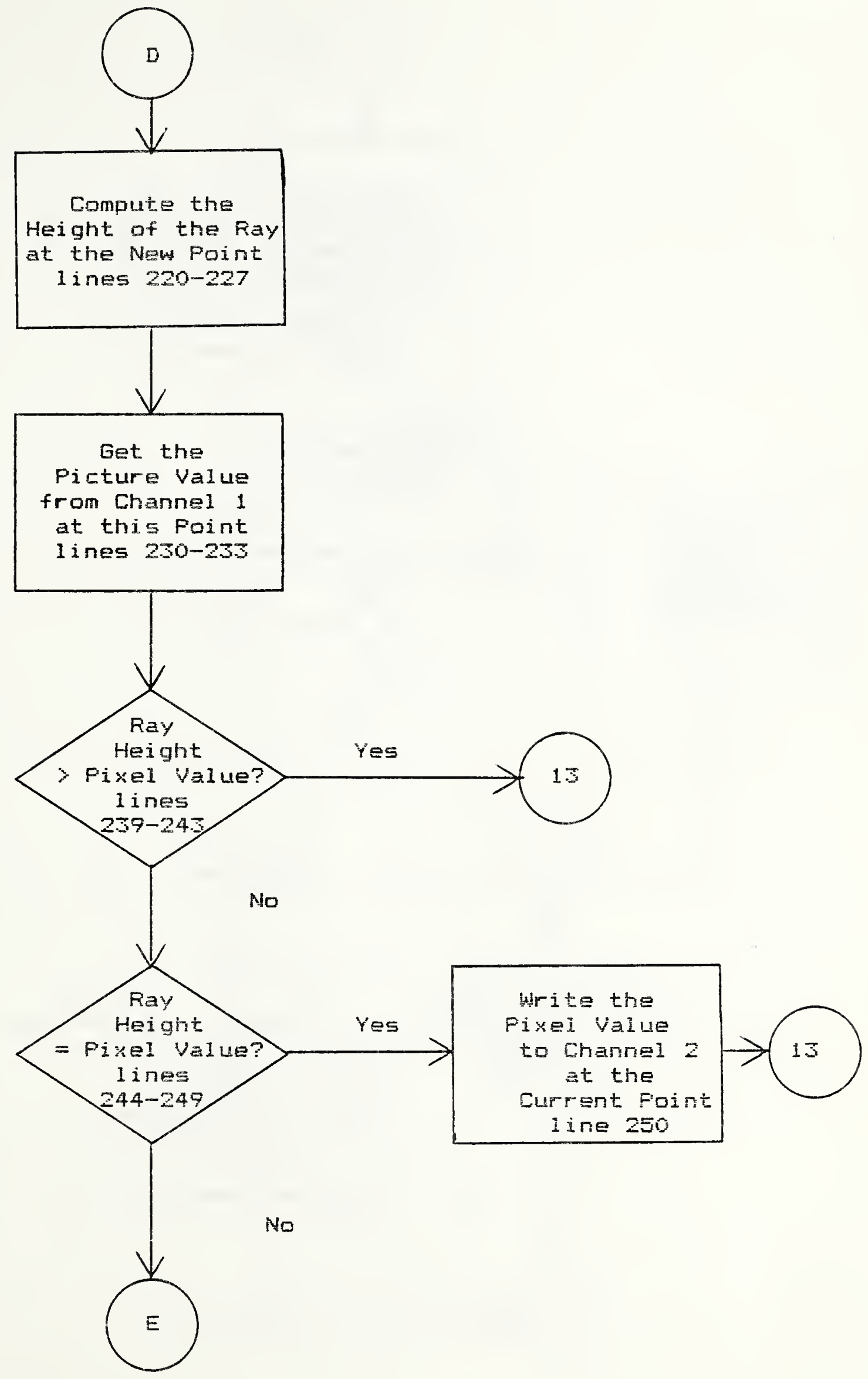



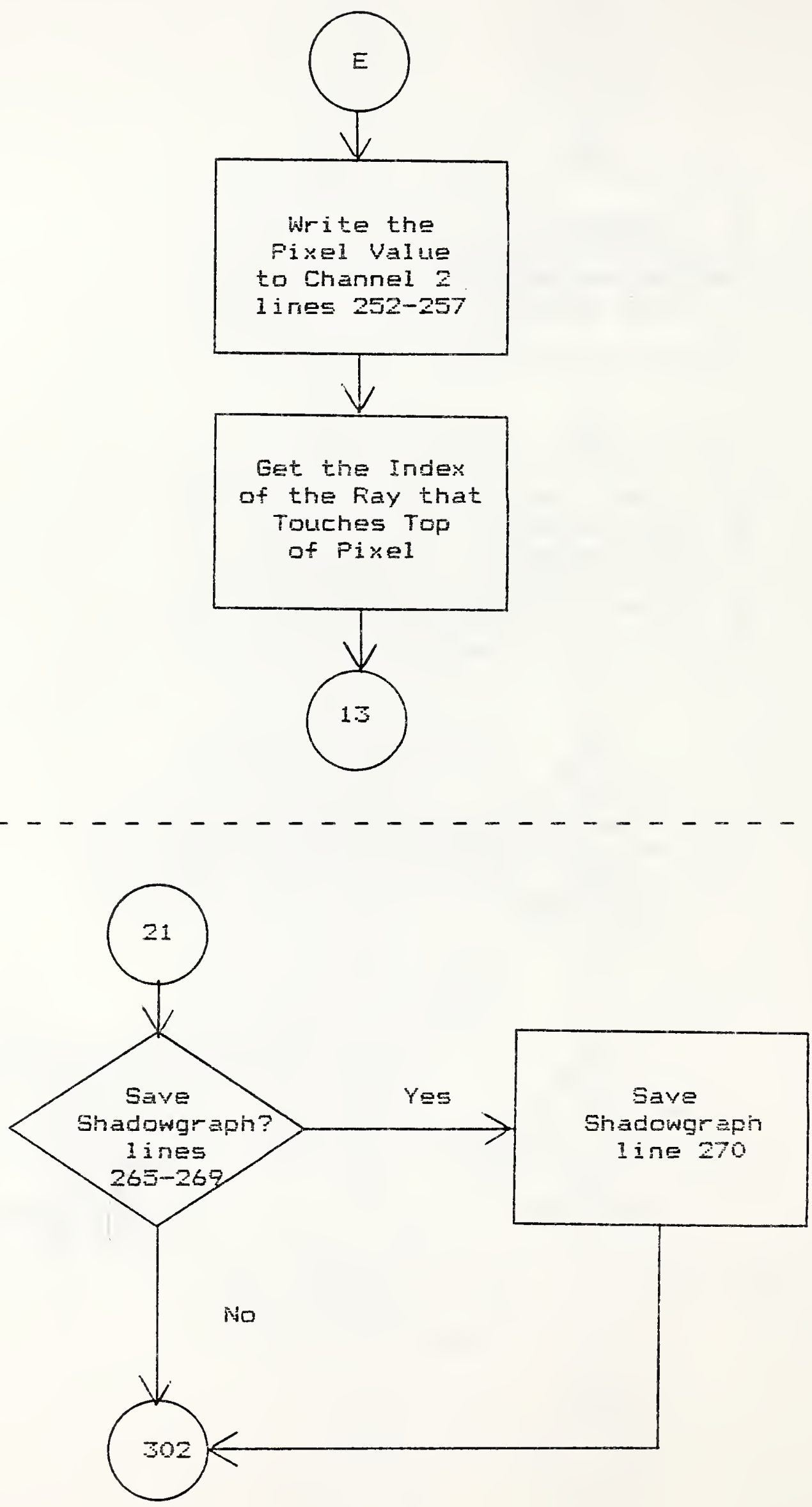


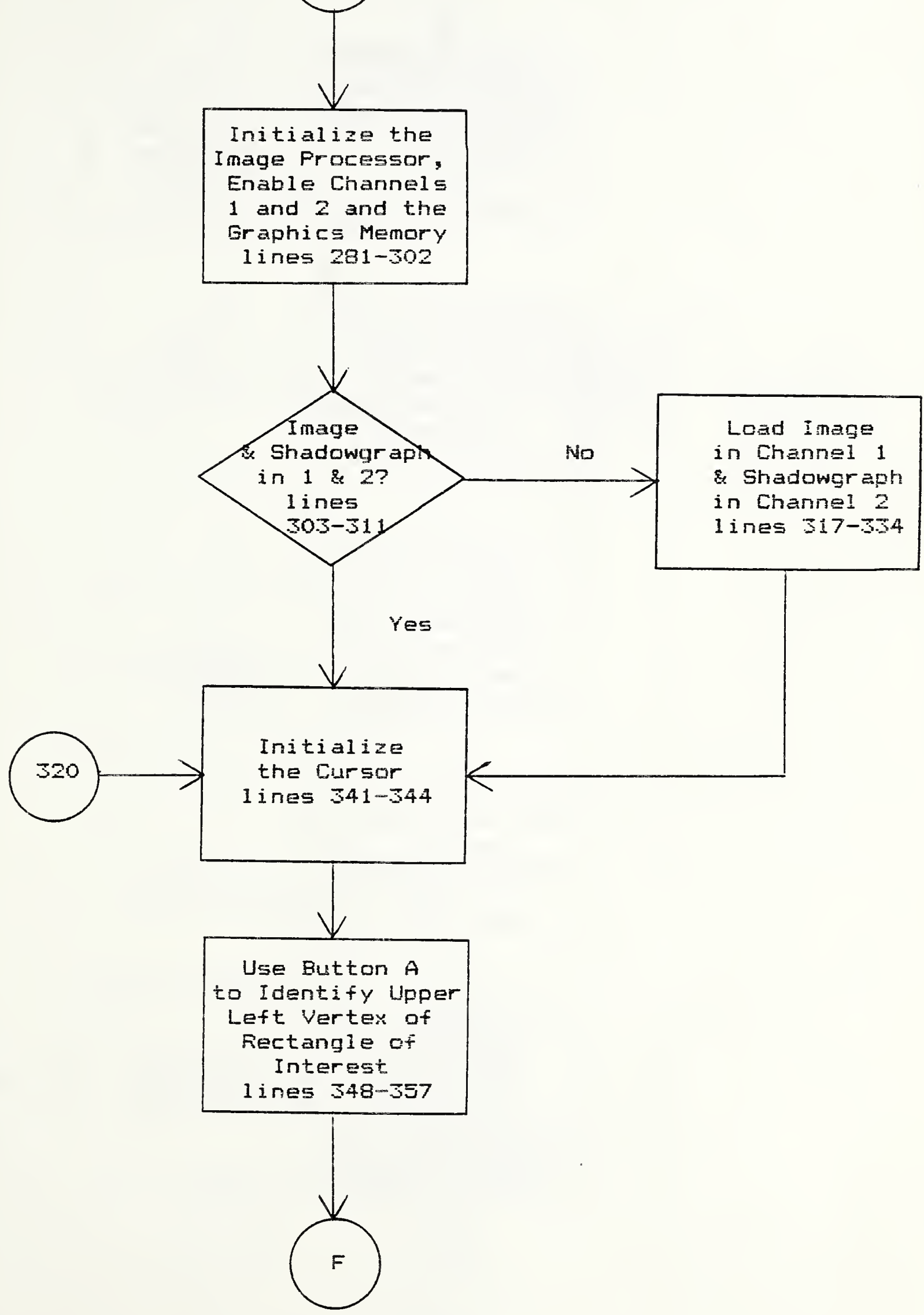




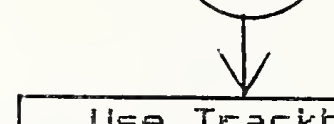

Use Traciball

Function Button A

to Identify Lower

Right Vertex of

Rectangle of

Interest

1 ines $360-364$

Set up Arrays

Representing

Vertex Vectors

of the Rectanglo of Interest

1 ines $367-382$

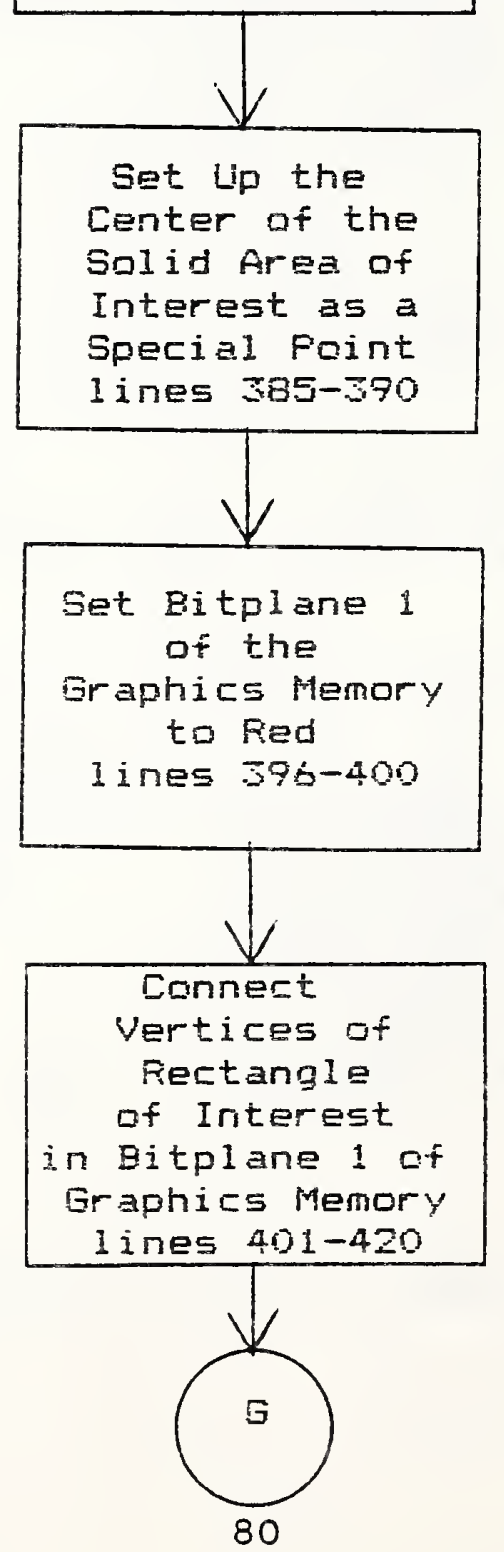




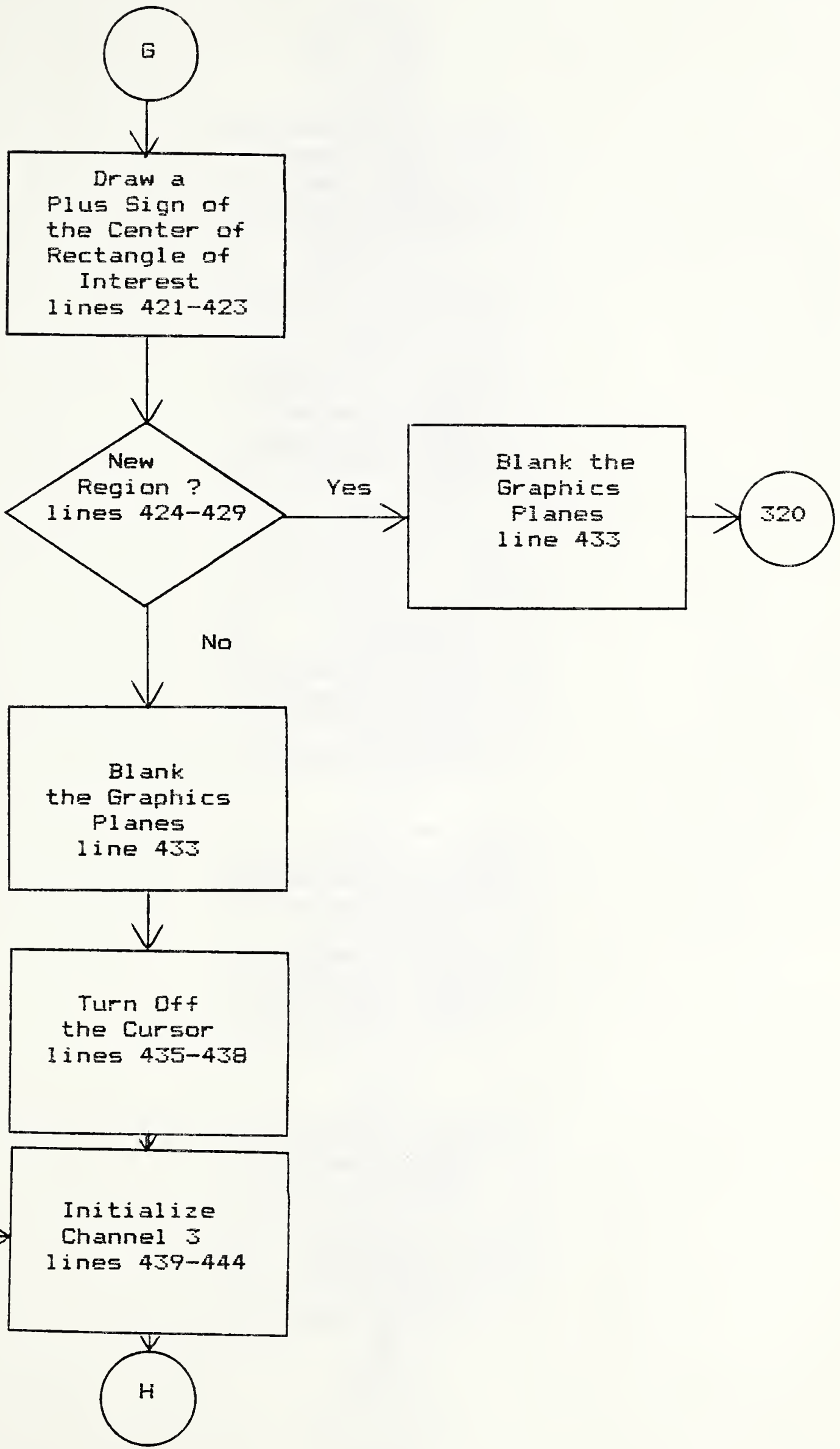



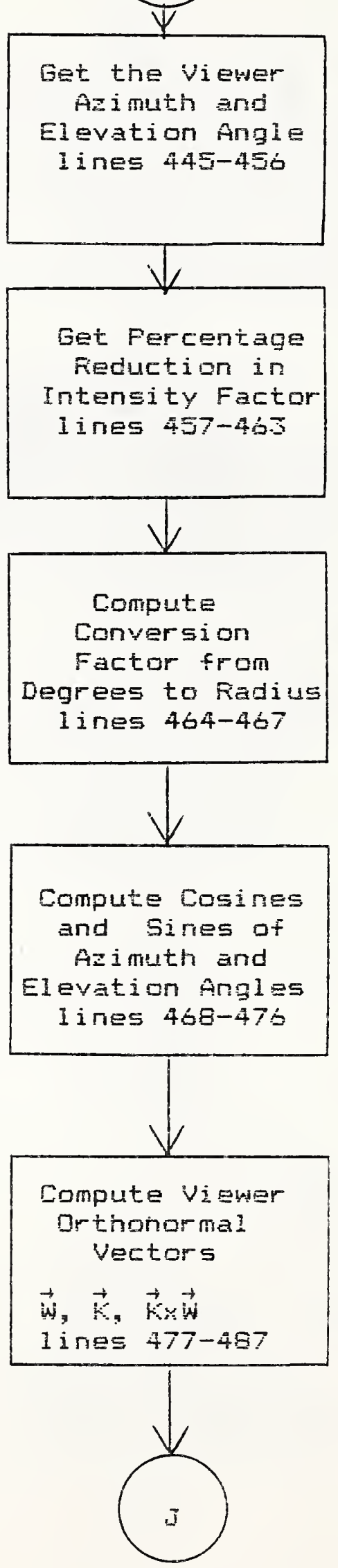


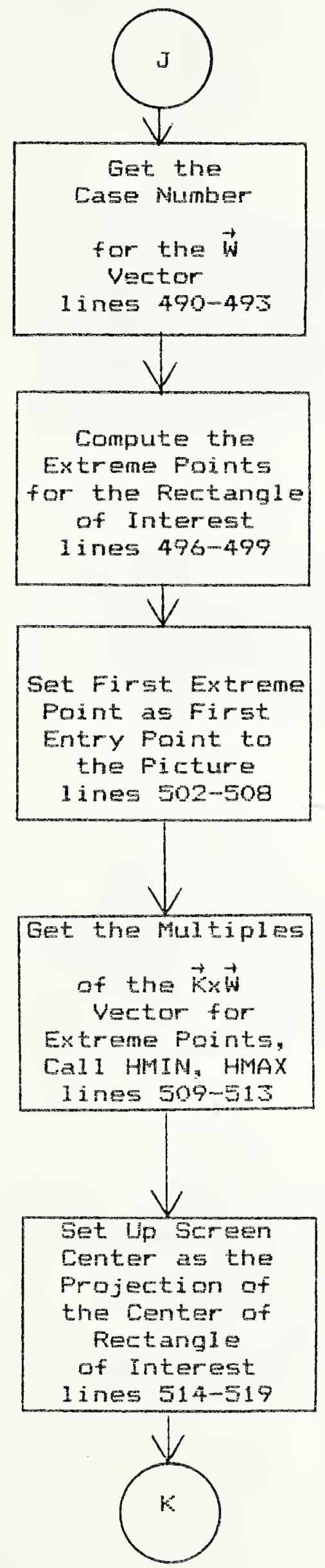

83 


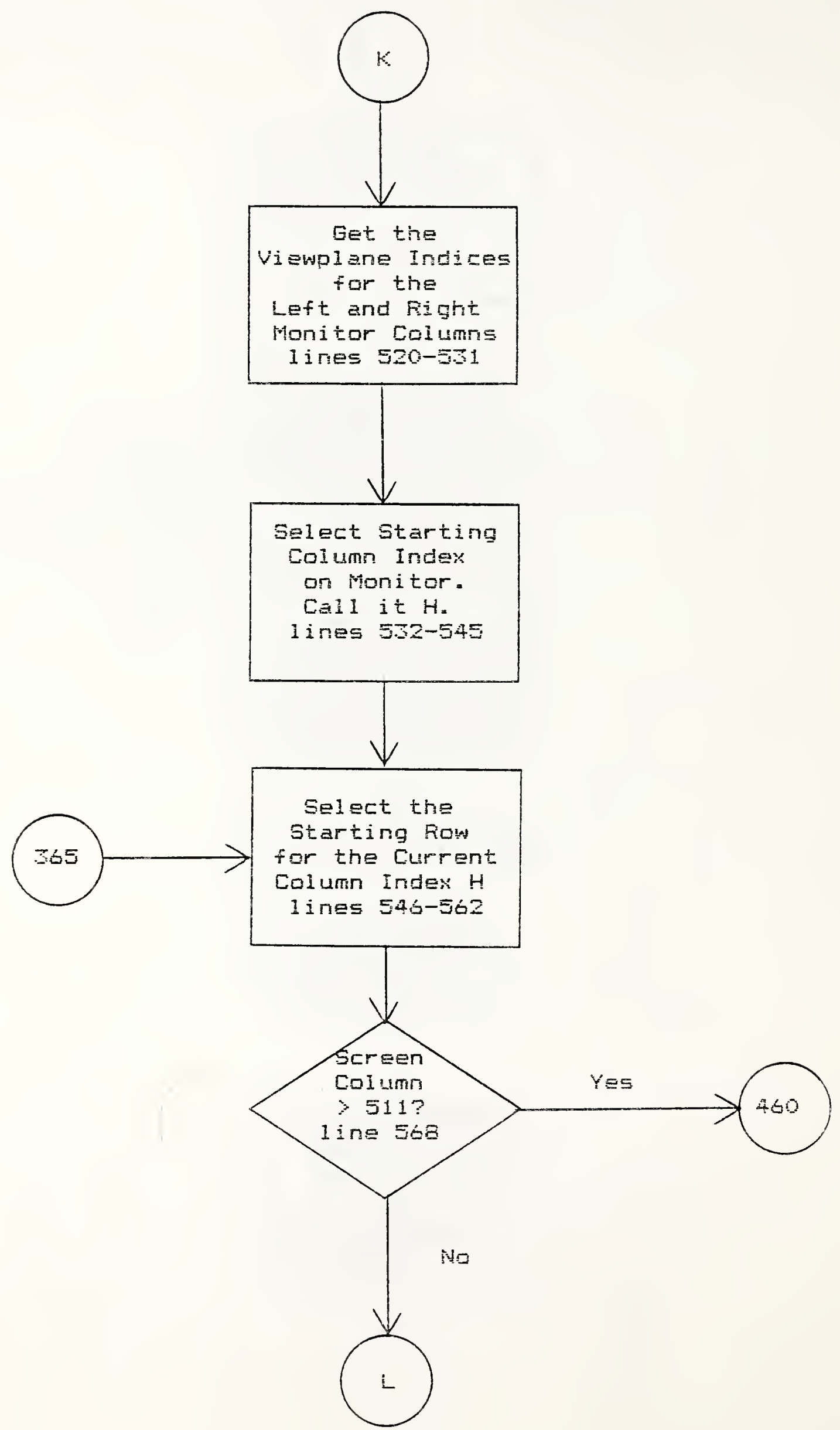




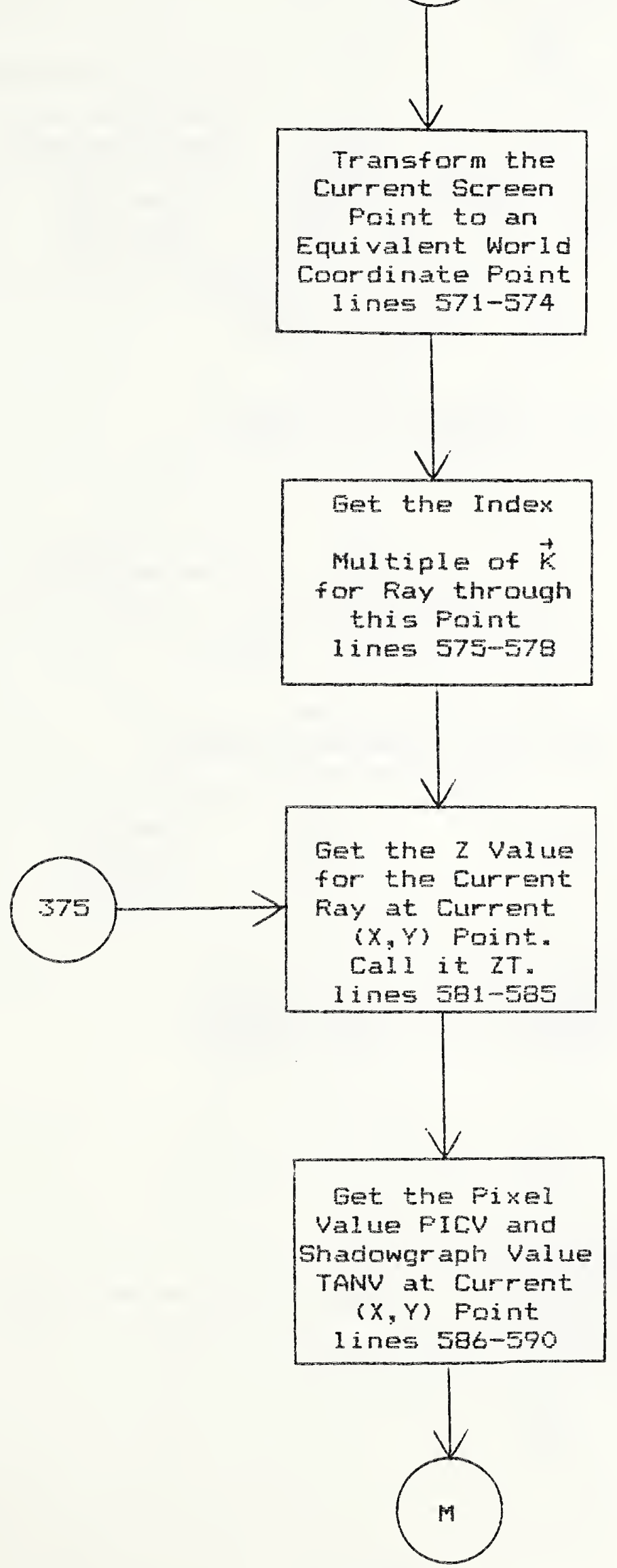




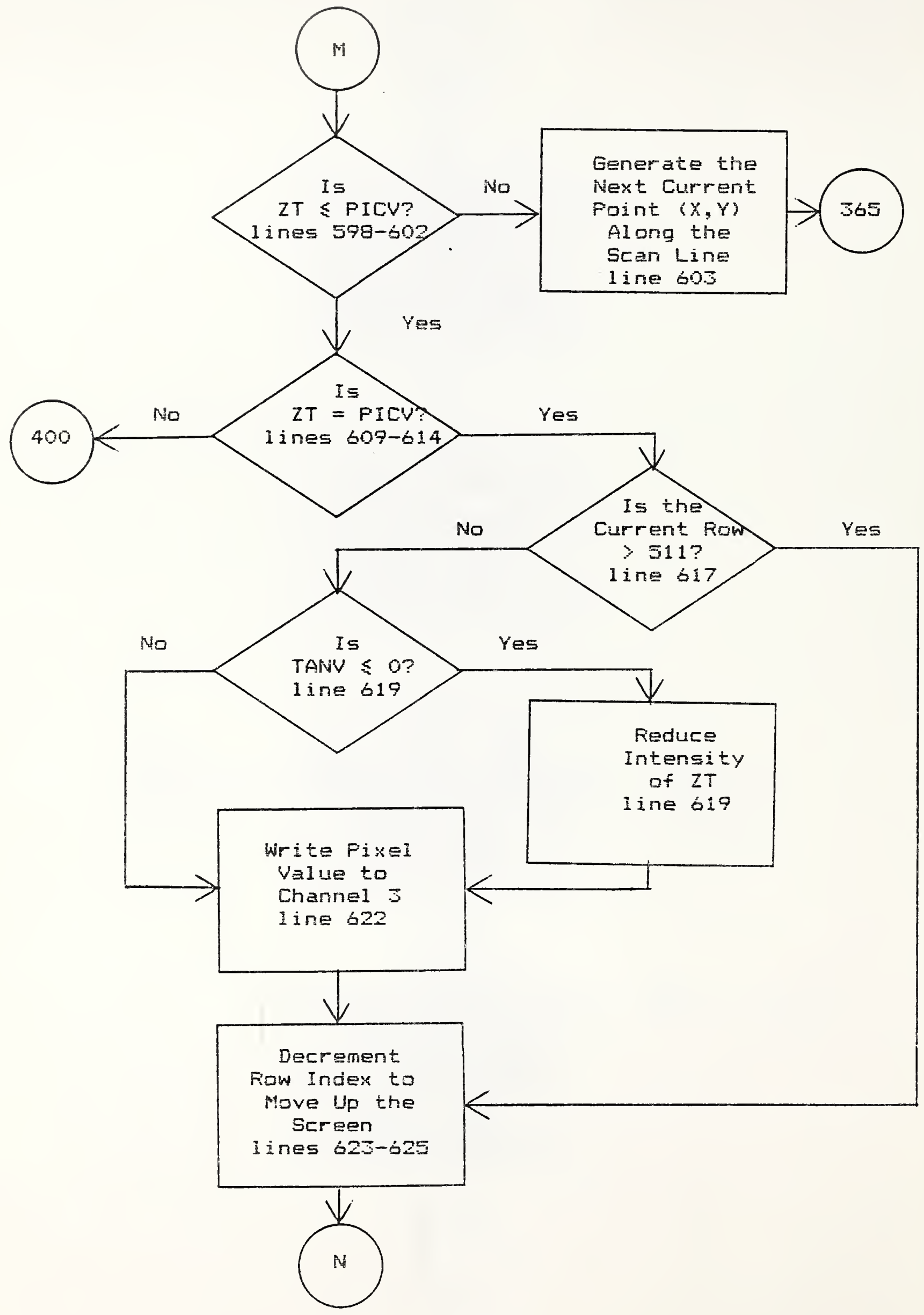




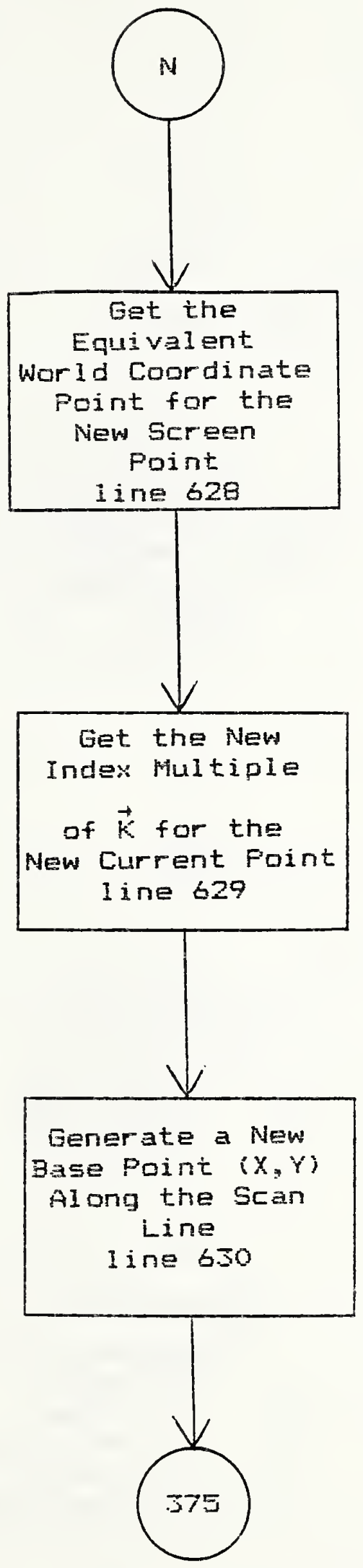




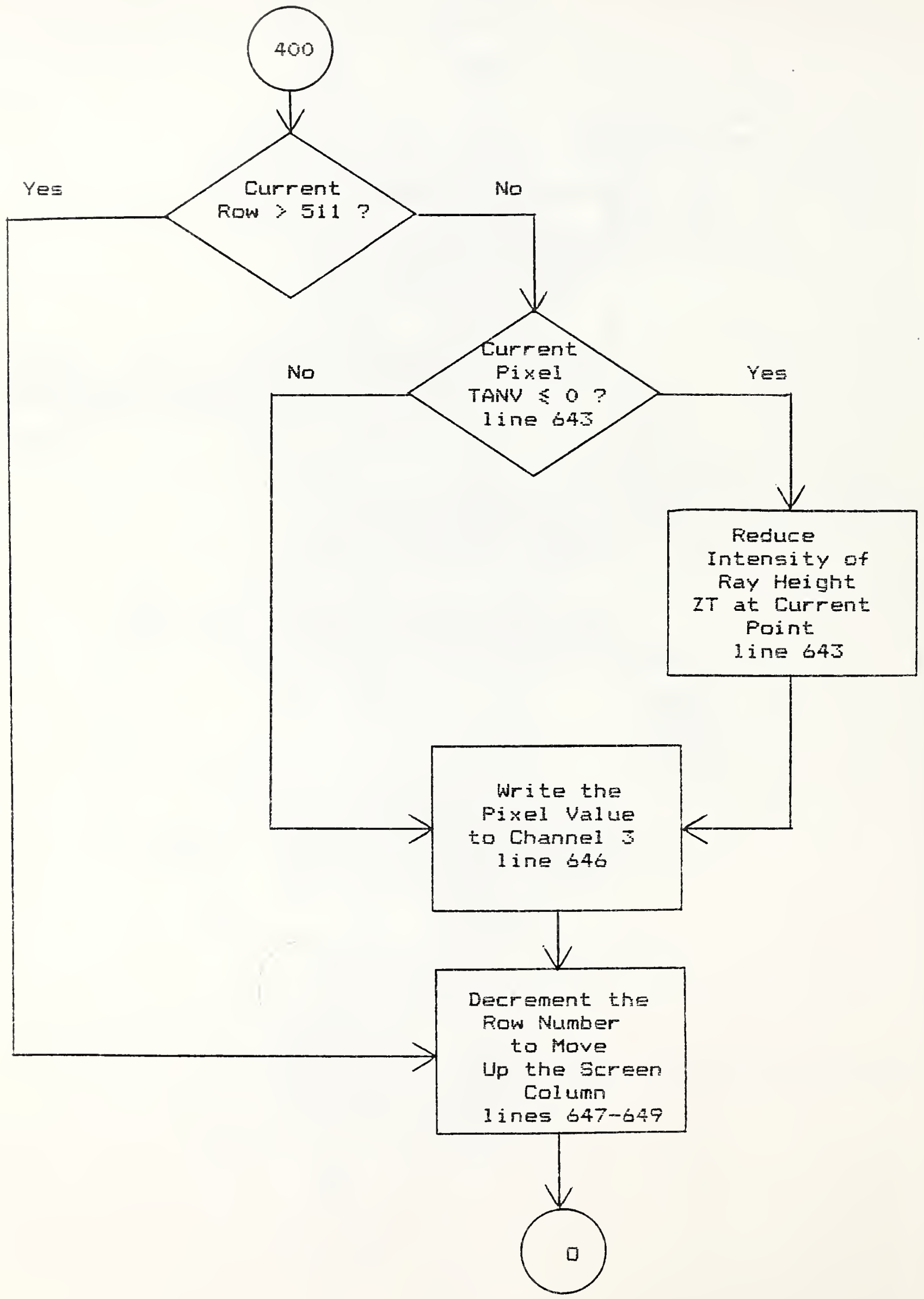




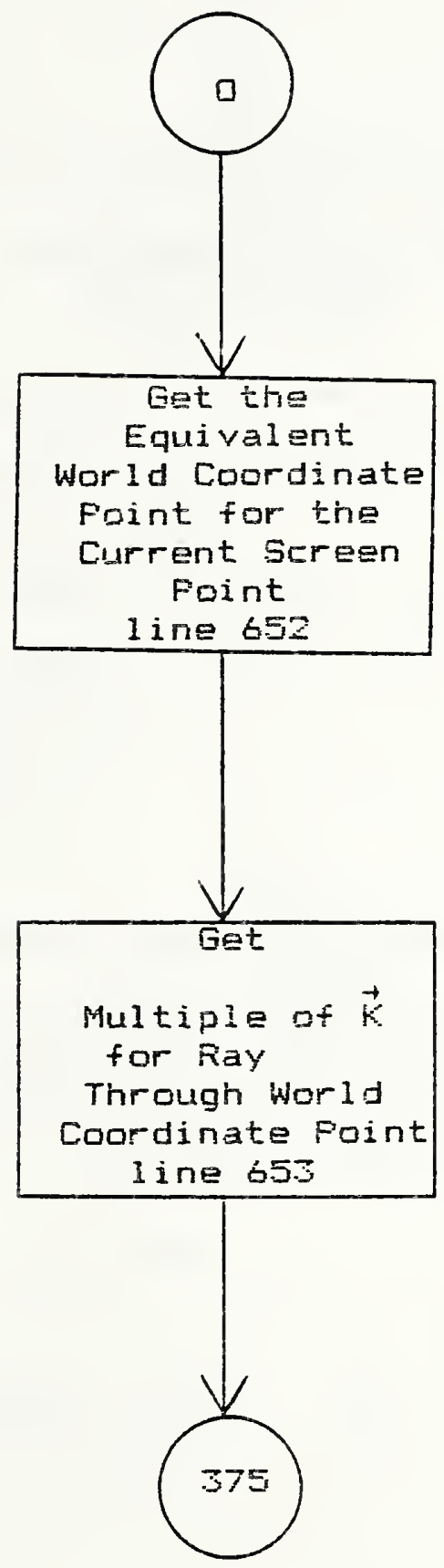




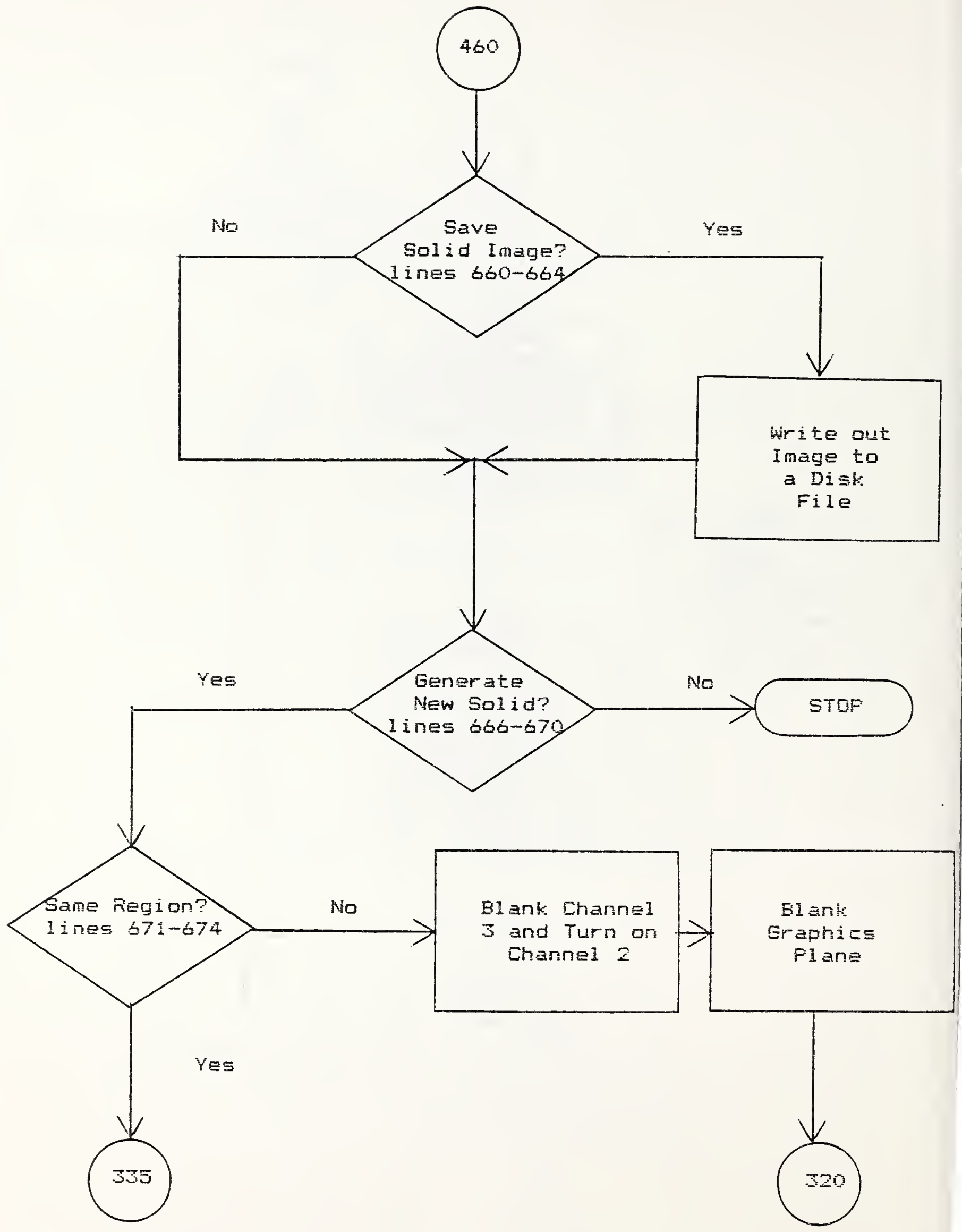




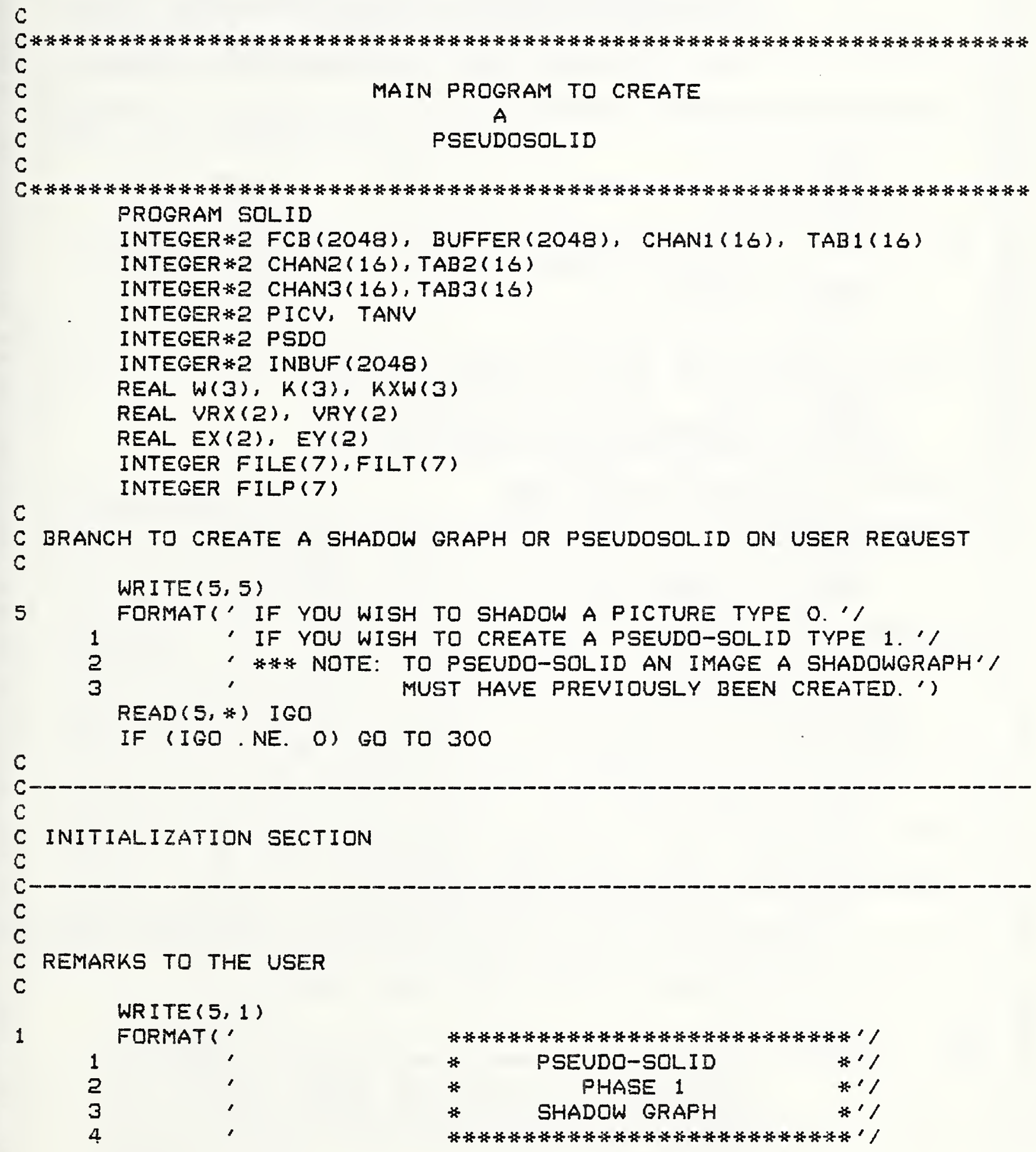




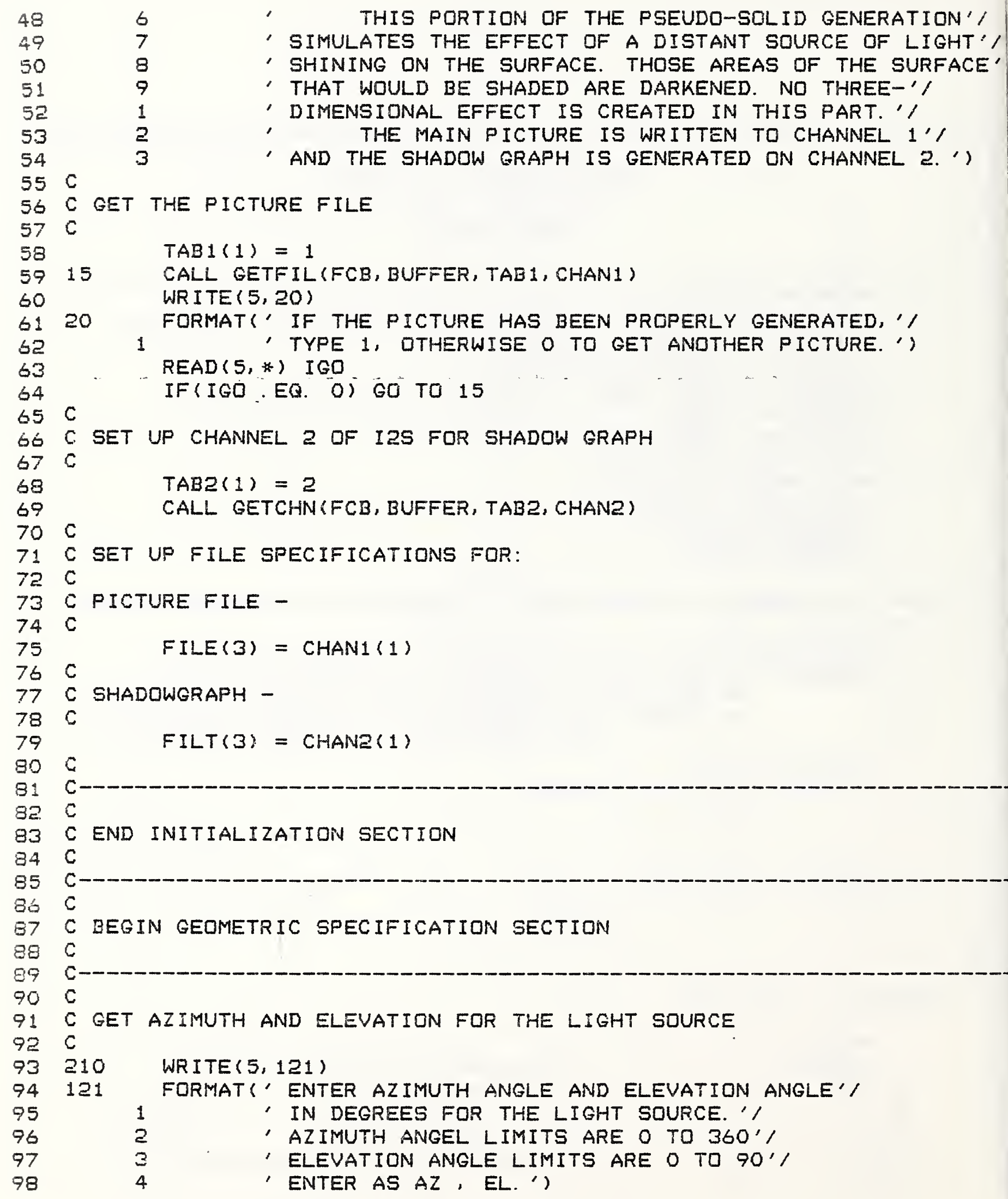


102 $103 \mathrm{C}$ $104 \mathrm{C}$ 105 $106 \mathrm{C}$ 107 $108 \mathrm{C}$ $109 \mathrm{C}$

110

111

112

113

114

115

116

117

$118 \mathrm{C}$

$119 \mathrm{C}$

$120 \mathrm{C}$

$121 \mathrm{C}$

122

123

C

$\operatorname{READ}(\zeta, *) \quad A L, E L$

IF ( AZ . LT. O. OR. AZ . GT. 360 . OR. EL. LT. O. . OR.

1 EL. GE. 90.) GO TO 210

SET UP CONVERSION FACTOR FROM DEGREES TO RADIANS

$$
\operatorname{CONV}=3.14159 / 180 \text {. }
$$

COMPUTE THE DIRECTION SINES AND COSINES FOR THE RAYS FROM THE LIGHT SOURCE TO THE SURFACE

$$
\begin{aligned}
& A Z=\operatorname{CONV*AZ} \\
& E L=\operatorname{CONY} A E L \\
& C E=\operatorname{COS}(E L) \\
& C A=\operatorname{COS}(A Z) \\
& S E=\operatorname{SIN}(E L) \\
& S A=\operatorname{SIN}(A Z)
\end{aligned}
$$

\section{$c$}

SET UP TWO UNIT VECTORS:

$$
\begin{aligned}
& W \text { - THIS UNIT VECTOR POINTS ALONG THE LIGHT } \\
& \text { R - THYS TOWARDS THE ORIGIN } \\
& \text { POINTS ACROSS A PLANE MADE OF LIGHT RAYS }
\end{aligned}
$$

C THESE COMMENTS ARE A NOTE ON THE UNDERLYING GEOMETRY.

C GIVEN AN AZIMUTH AND AN ELEVATION FOR THE LIGHT SOURCE ONE

C CAN THINK OF A PLANE FORMED BY ROTATING THE $X-z$ PLANE

C BY THE AZIMUTH ANGLE. NOW FILL UP THIS PLANE WITH LIGHT

C RAYS POINTING IN THE DIRECTION OF THE W-VECTOR. NOW

C CONSIDER A UNIT VECTOR IN THIS PLANE, CALLED $K$. THAT IS

C ORTHOGONAL TO W. THIS VECTOR IS THEN ORTHOGONAL TO ALL OF THE

C LIGHT RAYS POINTING IN THE DIRECTION W. EACH LIGHT RAY

C CAN BE INDEXED FROM A FIXED POINT ON THE PLANE BY ADDING

C SOME MULTIPLE OF THE K-VECTOR. FURTHERMORE FROM THAT SAME

C FIXED POINT ON THE PLANE ONE CAN ACCESS ANY POINT ON ANY

C LIGHT RAY IN THE PLANE BY ADDING A MULTIPLE OF $K$ AND THE

C ADDING A MULTIPLE OF W. FINALLY, ALL LIGHT RAYS IN THE

C DIRECTION W FALL ON SOME PLANE PARALLEL TO THE ROTATED PLANE

C ABOVE. IF WE TAKE THE CROSS PRODUCT OF $K$ AND W WE GET A

C VECTOR THAT CAN BE USED TD ACCESS ANY PLANE PARALLEL TO THE

C ROTATED PLANE. IN THIS PROGRAM THE MULTIPLES OF $K$ ARE

C THE V-VARIABLES. THE MULTIPLES OF W ARE R-VARIABLES

C AND THE MULTIPLES OF THE CROSS PRODUCT ARE THE H'S.

$$
\begin{aligned}
& W(1)=-C E * C A \\
& W(2)=-C E * S A \\
& W(3)=-S E \\
& K(1)=C A * S E \\
& K(2)=S A * S E \\
& K(3)=-C E \\
& K X W(1)=-S A \\
& K X W(2)=C A
\end{aligned}
$$


$151 \mathrm{C} \quad W R I T E(6,900) W(1), W(2), W(3), K(1), K(2), K(3), K X W(1), K \times W(2)$

152 C900 FORMAT(' $W=\prime, 3615.7, ' k=\prime, 3615.7, ' k \times w=\prime, 2615.7)$

$153 \mathrm{C}$

154 C GET THE W-VECTOR CASE INDEX

$155 \mathrm{C}$

156

157

158

$159 \mathrm{C}$

160

161

162

163

$164 c$

$165 \mathrm{C}$

$166 \mathrm{C}$

167

$168 \mathrm{C}$

$169 \mathrm{C9} 10$

$170 \mathrm{C}$

171

172

173

174

175

$176 \mathrm{C}$

177

CALL WCASE (W, IWCASE)

SET UP THE ENTIRE PICTURE FOR SHADOWING

C

$\operatorname{VRX}(1)=0$.
$\operatorname{VRY}(1)=0$.
$\operatorname{VRX}(2)=511$.
$\operatorname{VRY}(2)=511$.

GET THE EXTREME PQINTS

C

CALL EXTREM (IWCASE, VRX, VRY, EX, EY, IFLG)

WRITE (6, 910)EX(1), EY(1), EX(2), EY(2)

C10 FORMAT(' EXTREME PTS $1=$ ', 2615.7,' $2=\prime, 2615.7$ )

$178 \mathrm{C}$

179

180

181

182

183

184

185

186

137

188

189

190

191

192

193

194

195

196

197

198

$$
\begin{aligned}
& I X I N=E X(1) \\
& I Y I N=E Y(1) \\
& X I N=I X I N \\
& Y I N=I Y I N
\end{aligned}
$$

C TRANSFER THE PIXEL

SHADOWED

CALL RDPIC (FCB, FILE, PICV, IXIN, IYIN, 1, IERR)

CALL WRPIC (FCB, FILT, PICV, IXIN, IYIN, 1, IERR)

C WRITE (6, 925) IXIN, IYIN, PICV

C925 FORMAT(' FIRST EXT. PT. =',3I10)

$C$

C GET THE BOUNDARY POINT OF THE PICTURE WHERE THE PROUECTED W

C RAY ENTERS

C

9 CALL XYIN (IWCASE, EX, EY, IXIN, IYIN, XIN, YIN, IFLG)

$I X=I X I N$

$I Y=I Y I N$

$X=X I N$

$Y=Y I N$

C WRITE $(6,940) X, Y, \operatorname{IWCASE}, E X(1), E Y(1), E X(2), E Y(2)$

C940 FORMAT' BOUNDARY PT $=\prime, 2615.7, '$ CASE =', I5,' EX1, EY1, EX2, EY2=' C 1 4G15.7)

$$
1
$$

IF (IFLG. EQ. 1) STOP.' $W(1)=W(2)=0$ IN XYIN DURING SHADOW'

IF (IFLG. EQ. O) GO TO 10

IF (IFLG. EQ. 2) GO TO 21

$C$

C TRANSFER THE PIXEL VALUE OF THE ENTRY POINT TO THE BHADOWGRAPH C

10 CALL RDPIC (FCB, FILE, PICV, IX, IY, 1, IERR)

CALL WRPIC (FCB, FILT, PICV, IX, IY, $1, I E R R$ )

$Z=P I C V$ 
$104 \mathrm{C} \quad$ WRITE $(6,950)$ IX, IY, PICV

105 C950 FDRMAT(' BNDRY PT=', 3I10)

$206 \mathrm{C}$

207 C GET THE INDEX DF THE RAY THAT INTERCEPTS THE POINT $(X, Y, Z)$

$208 \mathrm{C}$

09

$10 \mathrm{C}$

CALL GETV $(X, Y, Z, K, V)$

WRITE (6, 960) $V$

FORMAT (' BDRY RAY INDEX=', G15.7)

$111 \mathrm{c}$

$112 \mathrm{C}$

:13 C GET THE NEXT POINT ALONG THE SCAN LINE

$114 \mathrm{C}$

1513

$116 \mathrm{C}$

$117 \quad 6970$

CALL GNXY $(X, Y, I X, I Y, W, I F L G)$

WRITE (6, 970) $X, Y, I X, I Y$

FORMAT(' NEXT POINT =', 2G15.7, 2I10)

IF (IX.LT. O . OR. IX . GT. 511) GO TO 8

IF (IY. LT. O . DR. IY . GT. 511) GO TO 8

18

$! 19$

.200

:21 C THE MODEL USED HERE ASSUMES THAT THE RAY SCIMS THE TOP OF A

:22 C PIXEL THAT IT SEES, SINCE PIXELS ARE ASSUMED TO BE POINTS

NOW COMPUTE THE HEIGHT DN THE CURRENT RAY INDEXED BY $V$ AT THE

$C$ POINT $(X, Y)$

$226 \mathrm{C}$

CALL $G E T Z(X, Y, V, K, Z T)$

WRITE $(6,980) Z T$

229 C980 FORMAT(' $Z T=\prime, G 15.7)$

$230 \mathrm{C}$

gET THE PICTURE VALUE AT THE CURRENT POINT (IX, IY)

CALL RDPIC (FCB, FILE, PICV, IX, IY, 1, IERR)

COMPARE THIS VALUE AGAINST THE RAY HEIGHT, ZT, AT THIS POINT TO DETERMINE WHETHER THE RAY SEES THE POINT

C

$$
P=P I C V
$$

CASE 1: IF ZT > PICV THEN THE PIXEL IS NOT VISIBLE TO THIS RAY CONTINUE TRACING THIS RAY.

IF ( $Z T . G T . P+1 . E-5)$ GO TO 13

CASE 2: IF ZT . EQ. PICV THEN THE POINT IS VISIBLE, WRITE THE PIXEL OUT TO THE SHADOWGRAPH BUT CONTINUE TRACING THE SAME RAY

IF ( $Z T$. LT. $P-1 . E-5)$ GO TO 19

CALL WRP IC (FCB, FILT, PICV, IX, IY, 1, IERR)

GO TO 13

C CASE 3: IF ZT C PICV THEN THE PIXEL VALUE IS SEEN BY THE RAY WRITE IT OUT AND GET THE FIRST RAY THAT SATISFIES ZT = PICV 


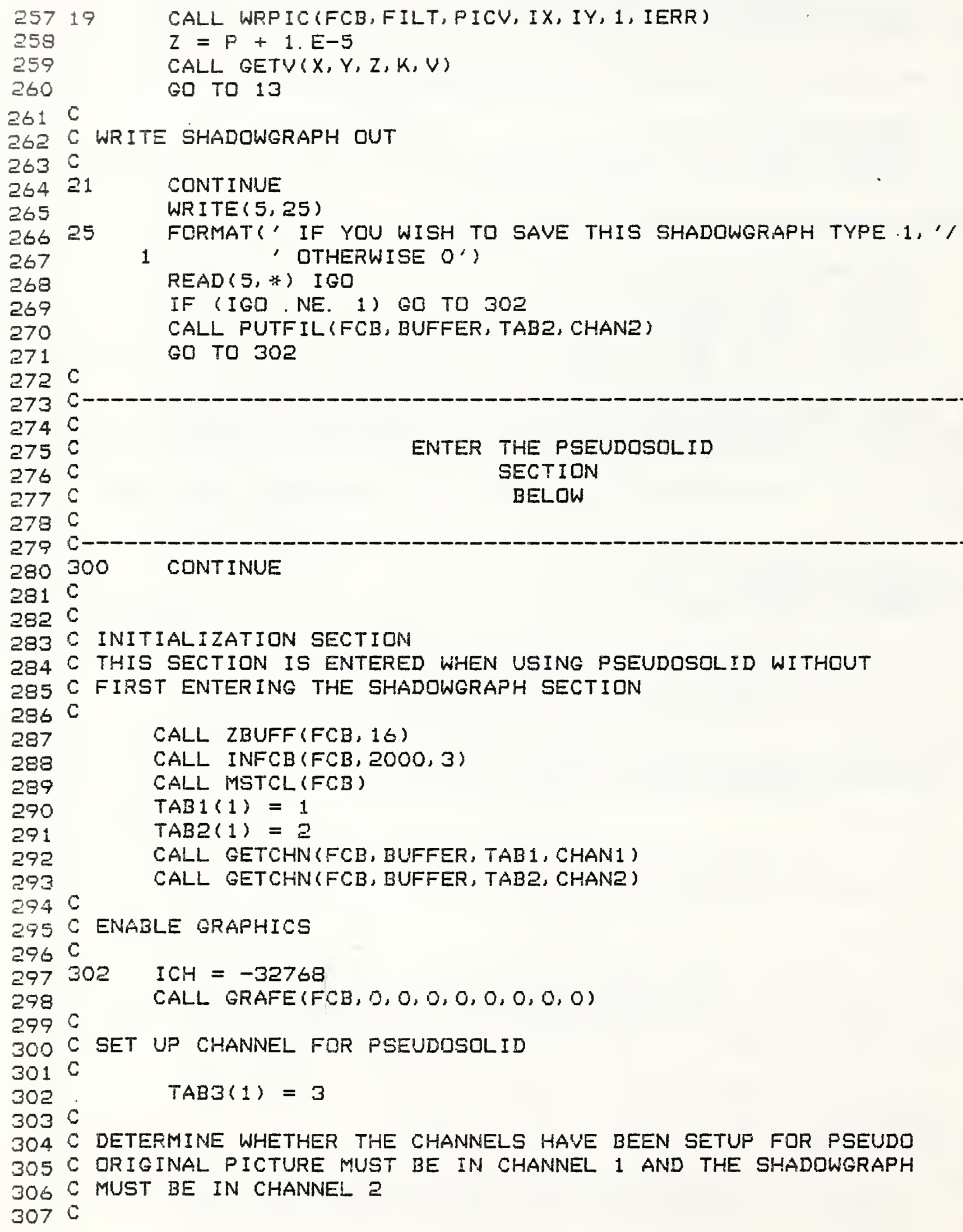




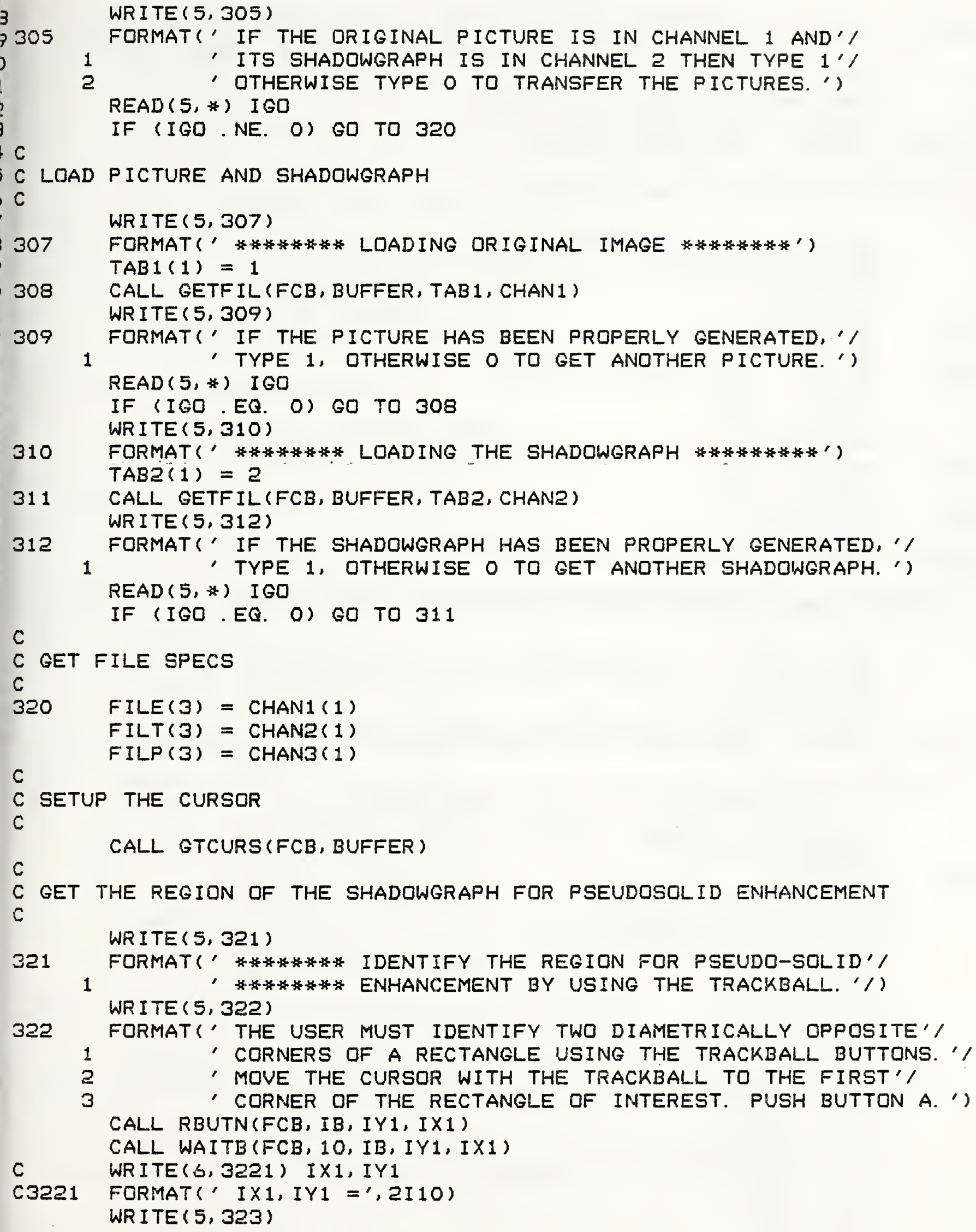




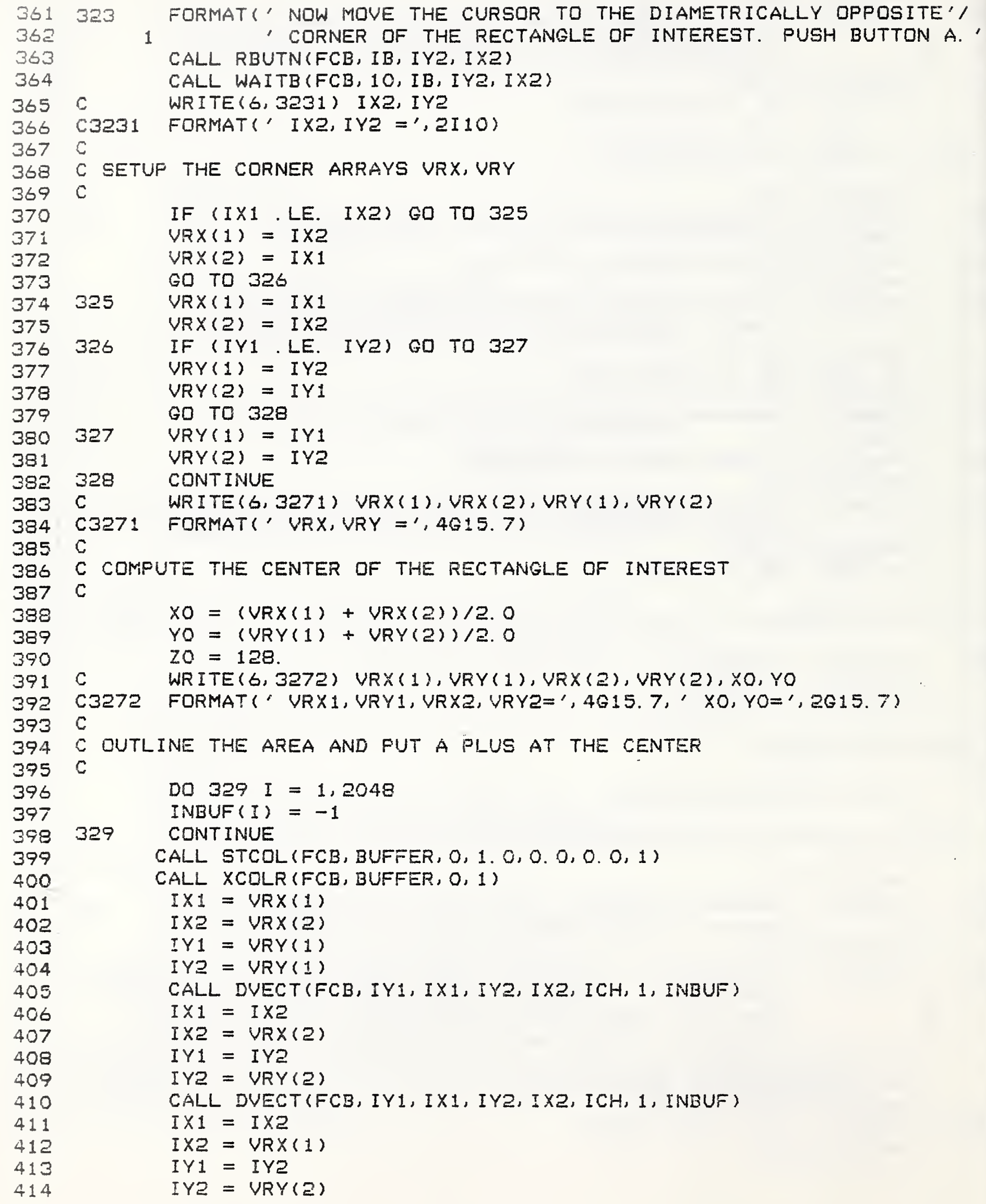




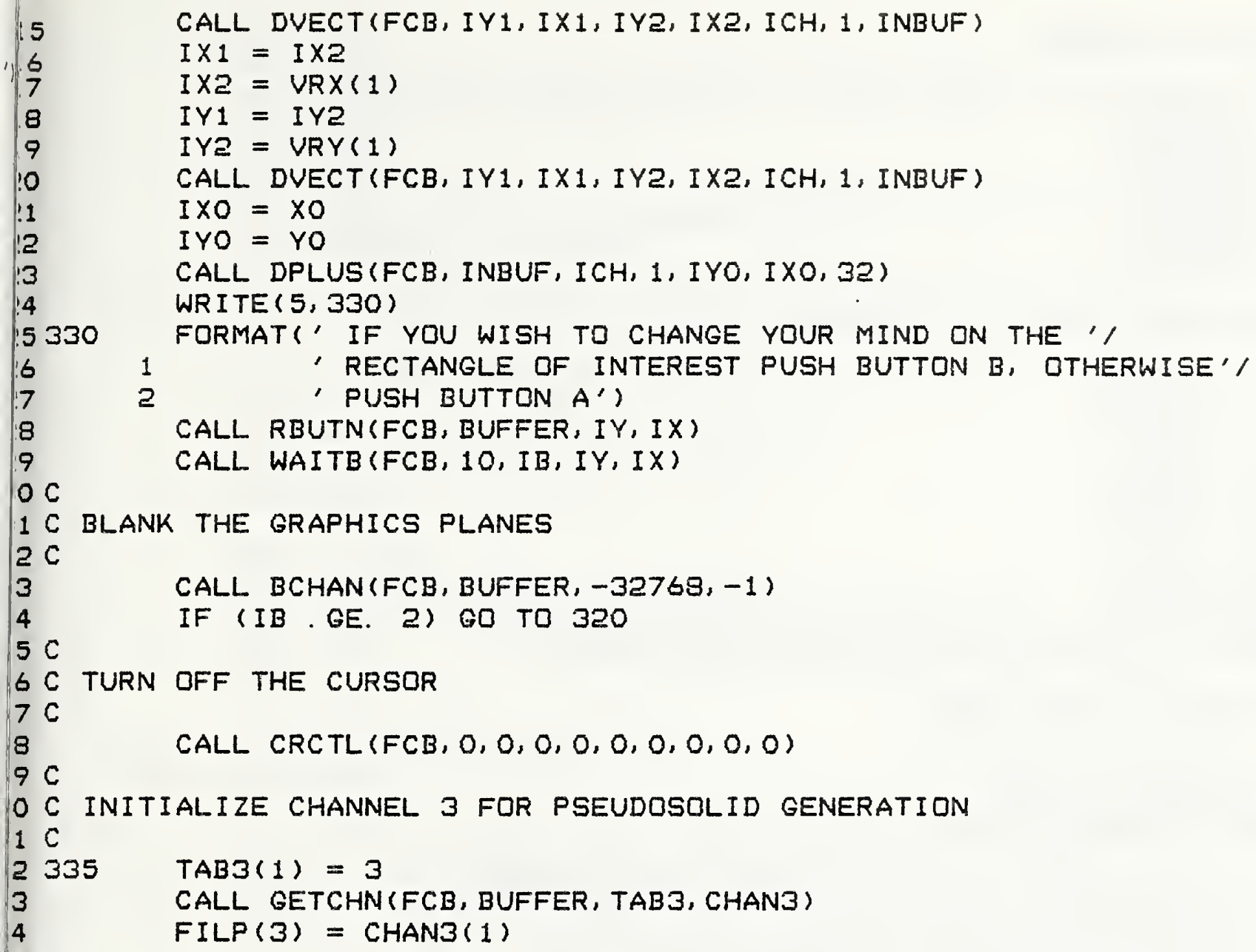

GET THE AZIMUTH AND ELEVATION DF THE VIEWING ANGLE C

340

WRITE (5, 341)

341

FORMAT\&" ENTER AZIMUTH ANGLE AND ELEVATION ANGLE',

1 ' IN DEGREES FOR THE VIEWER. AZIMUTHE ANGLE',

2 ' LIMITS ARE O TO 360. ELEVATION ANGLE LIMITS',

3 ' ARE O TO 90.'

4 'ENTER AS AZ, EL.')

$\operatorname{READ}(5, *) \quad A Z, E L$

IF (AZ . LT. O. . OR. AZ . GT. 360. . OR. EL. . LT. O. . OR.

1 EL.GE.,$O$, GO TO 340

C

C GET THE PERCENTAGE REDUCTION FOR SHADOWING

C

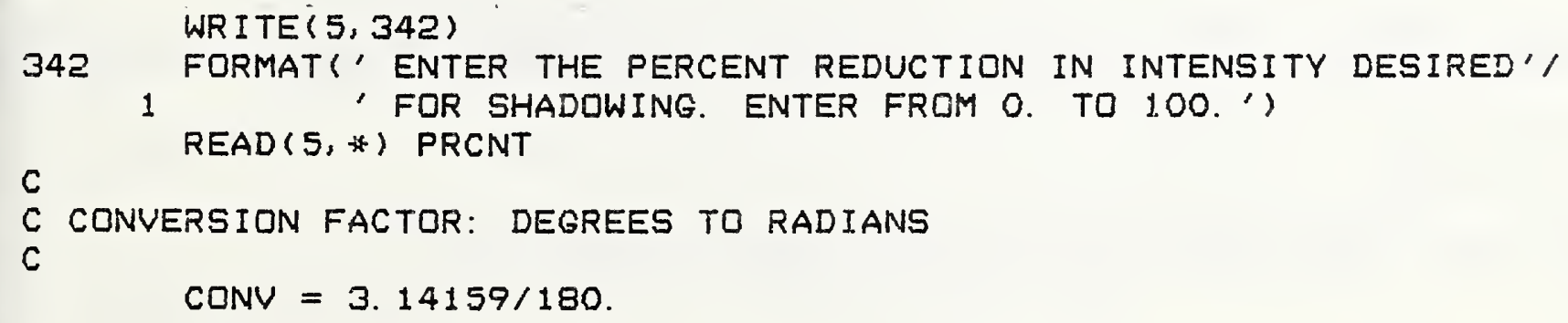


$\$ 69 \mathrm{C}$ GET SINES AND COSINES

$+70 \mathrm{C}$

$\$ 71$

$+72$

$A Z=C O N V * A Z$

$E L=C O N V * E L$

$C E=\operatorname{COS}(E L)$

$C A=\cos (A Z)$

$S E=\operatorname{SIN}(E L)$

774

778C SET UP THE VIEWER FRAME OF REFERENCE

$479 \mathrm{C}$

480

481

482

783

484

485

486

487

$488 \mathrm{C}$

$489 \mathrm{C} 345$

$490 \mathrm{C}$

$491 \mathrm{C}$ GET THE W-VECTOR CASE INDEX

$492 \mathrm{C}$

493

$494 \mathrm{C}$

$W(1)=-C E * C A$

$W(2)=-C E * S A$

$W(3)=-S E$

$K(1)=C A * S E$

$K(2)=S A * S E$

$K(3)=-C E$

$K X W(1)=-S A$

$K X W(2)=C A$

WRITE (6, 345) W(1),W(2),W(3),K(1),K(2),K(3),KXW(1),KXW(2)

FORMAT(' $W 1, W 2, W 3, k 1, k 2, K 3, K \times W 1, K X W 2=\prime, 8 G 15.7)$

495 (346 FORMAT!' IWCASE $=$ ', I4)

$496 \mathrm{C}$

$497 \mathrm{C}$ GET THE EXTREME POINTS FOR THE PSEUDOSCLID RECTANGLE

$498 \mathrm{C}$

499

$500 \mathrm{C}$

$501 C 347$

$502 \mathrm{C}$

$503 \mathrm{C}$ SET UP THE FIRST ENTRY POINT TO THE PICTURE

$504 \mathrm{C}$

505

506

507

508

$509 \mathrm{C}$

$510 \mathrm{C}$

$511 \mathrm{C}$

512

513

$514 \mathrm{C}$

$515 \mathrm{C}$

$516 \mathrm{C}$

$517 \mathrm{C}$

518

519

$520 \mathrm{C}$

521 C GET THE LIGHT PLANE INDEX FOR THE LEFT HAND COLUMN CF THE

$$
\begin{aligned}
& I X I N=E X(1) \\
& I Y I N=E Y(1) \\
& X I N=I X I N \\
& Y I N=I Y I N
\end{aligned}
$$

GET THE MULTIPLES OF THE KXW VECTOR FOR THE EXTREME POINTS

CALL GETH(EX(1), EY(1), O. , KXW, HMIN)

CALL GETH(EX(2), EY(2), O. , KXW, HMAX)

SET UP THE SCREEN CENTER FOR THE PROJECTION OF THE RECTANGLE

OF INTEREST CENTER. NOTE THESE ARE IN SCREEN COORDINATES. $X$ AND Y ARE REVERSE:

$$
\begin{aligned}
& \text { SXO }=256 . \\
& \text { SYO }=256 .
\end{aligned}
$$

522 C MONITOR

$523 \mathrm{C}$ 


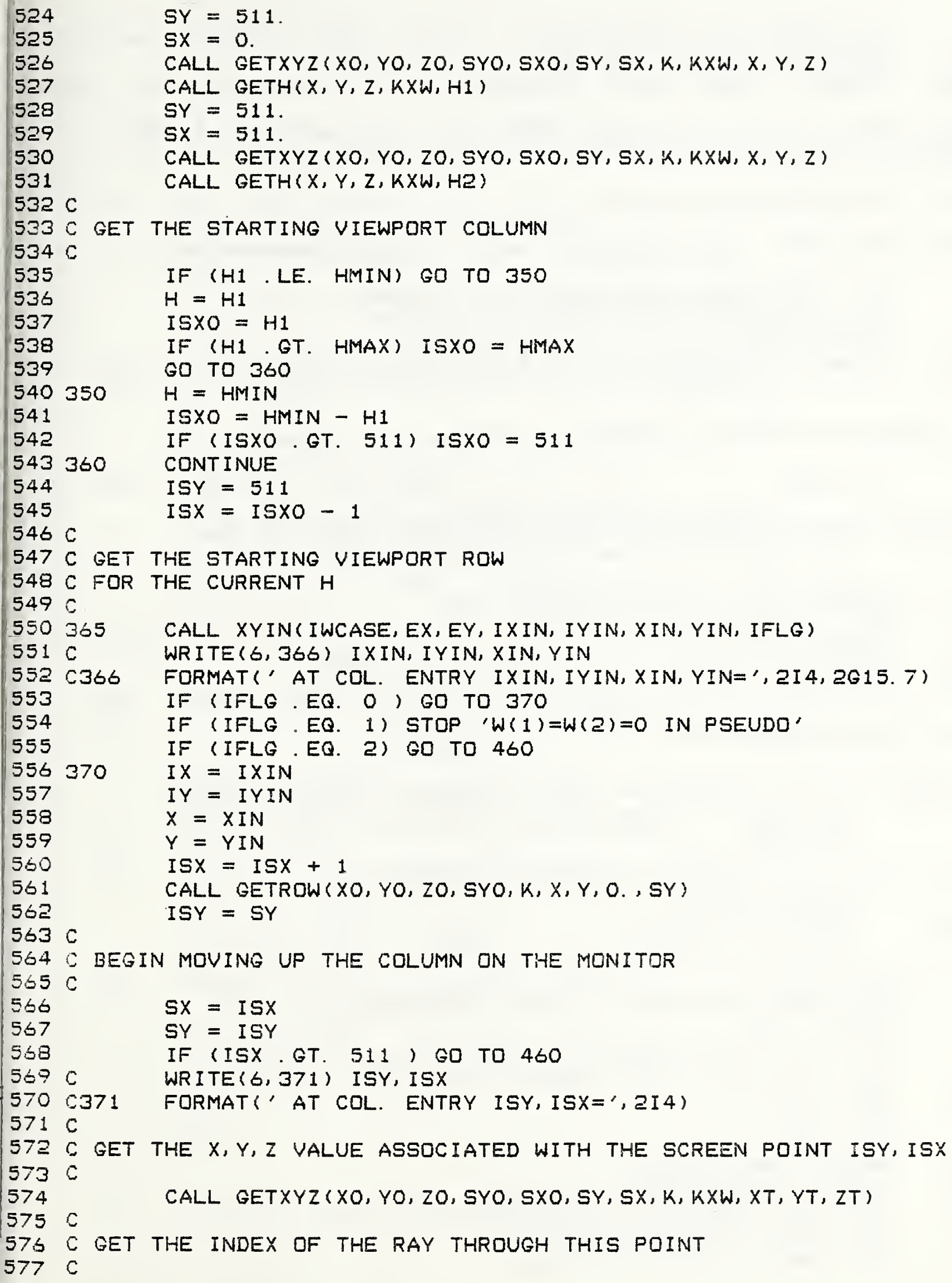

564 C BEGIN MOVING UP THE COLUMN ON THE MONITDR

$572 C$ GET THE $X, Y, Z$ VALUE ASSOCIATED WITH THE SCREEN POINT ISY, ISX 


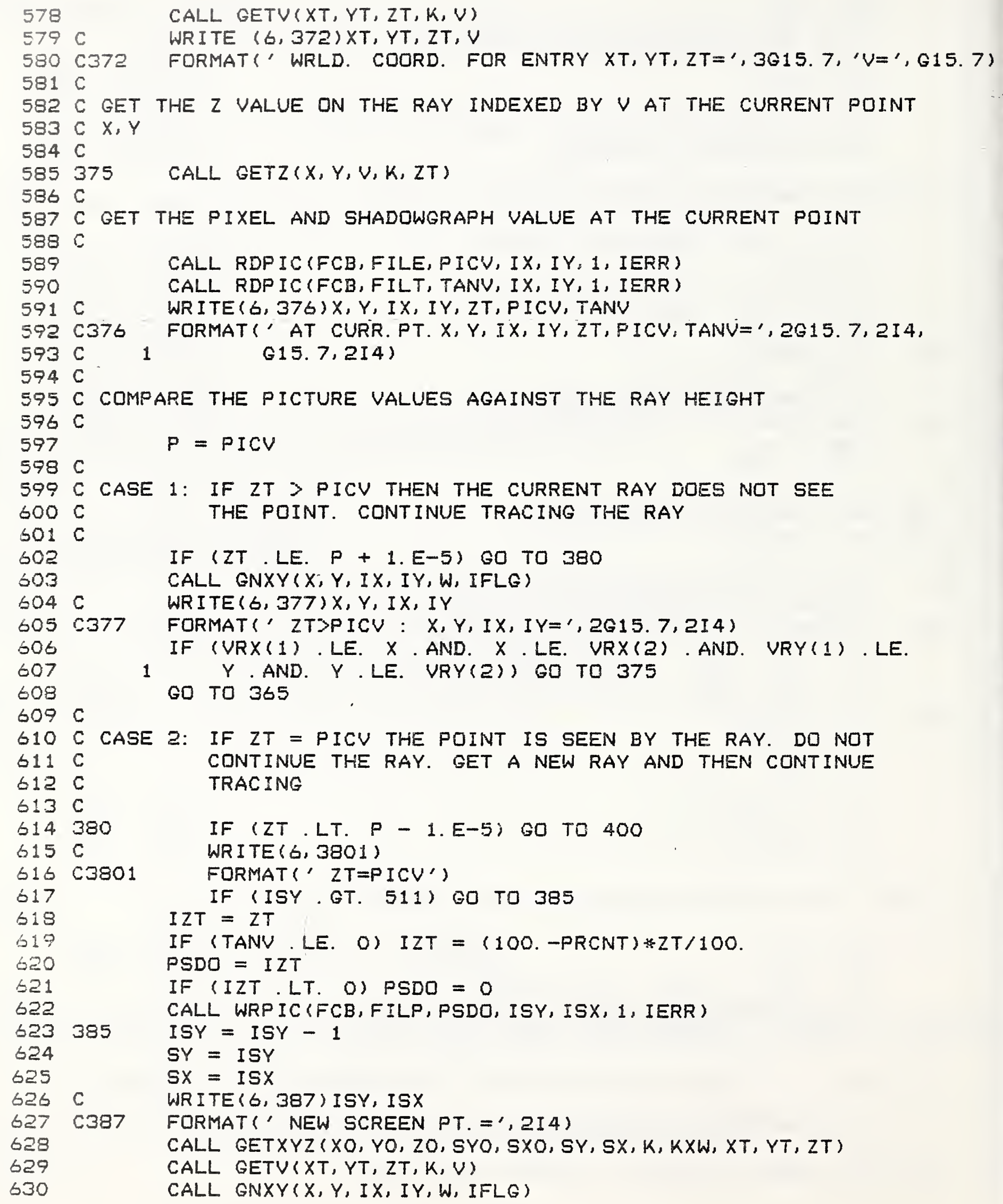
$612 C$ TRACING

IF (ZT. LE. $P+1 . E-5)$ GO TO 380

CALL GNXY $(X, Y, I X, I Y, W, I F L G)$

GO TO 365 
$631 \mathrm{C} \quad$ WRITE(6,386)XT,YT, ZT, V, X, Y, IX, IY

632 C386 FORMAT(' WRLD. COORD. FOR CURR.PT. $X, Y, Z=\prime, 3 G 15.7, ' V=\prime, G 15.7$, $633 \mathrm{C} \quad 1$ 634 GO TO 375

$635 \mathrm{C}$

636 C CASE 3: IF ZT \& PICV THE PIXEL IS SEEN BY THE RAY BUT DO NDT $637 C$

$638 \mathrm{C}$

639400 IF (ISY. GT. 511) GO TO 450

$640 \mathrm{C}$ WRITE $(6,401)$

641 C401 FORMAT(' ZTEPICV')

$642 \quad I Z T=Z T$

643 IF (TANV. LE. O) IZT $=(100 .-P R C N T) * Z T / 100$.

$644 \quad P S D O=I Z T$

645 IF (IZT. LT. O) PSDO =0

646 CALL WRPIC (FCB, FILP, PSDO, ISY, ISX, 1, IERR)

$647450 \quad$ ISY $=$ ISY -1

$648 \quad S Y=I S Y$

$649 \quad 5 X=I S X$

$650 \mathrm{C}$ WRITE $(6,451)$ ISY, ISX

651 C451 FORMAT(' NEW SCREEN POINT=', 2I 4)

652

653

$654 \mathrm{C}$

CALL GETXYZ (XO, YO, ZO, SYO, SXO, SY, SX, K, KXW, XT, YT, ZT)

CALL GETV $\left(X T, Y T^{\circ}, Z T, K, V\right)$

655 C 452 FORMAT(' WRLD. COORD. XT, YT, ZT=', 3G15. 7, ' $V=\prime, G 15.7$ )

656 GO TO 375

$657 \mathrm{C}$

$658 \mathrm{C}$ WRITE OUT PSEUDOSOLID PICTURE

$659 \mathrm{C}$

$660460 \quad$ WRITE $(5,470)$

661 470 FORMAT', IF YOU WISH TO SAVE THE PSEUDOSOLID IMAgE TYPE'/

662

663

1

READ (5, *) IGO

IF (IGO. NE. 1) GO TO 475

664

665

$566 \quad 475$

CALL PUTFIL (FCB, BUFFER, TAB3, CHAN3)

667480

068

669

670

671

672485 WRITE (5, 480)

FORMAT', IF YOU WISH TO GENERATE ANOTHER SOLID TYPE 1, ',

1

$\operatorname{READ}(5, *)$ IGO

IF ( IGO.EQ. O) STOP

673

WRITE $(5,485)$

678

FORMAT'' IF YOU WANT THE SAME REGION-OF-INTEREST TYPE 1, '/

1

$\operatorname{READ}(5, *)$ IM

IF (IM . EQ. O) GO TO 320

675

676

GO TO 335

677

STOP

678

END 


\section{3.1 Summary}

This subroutine intializes the programmable cursor at the center point of the screen. The calling sequence is:

CALL GTCURS (FCE, BUFFEF).

The parameters passed are:

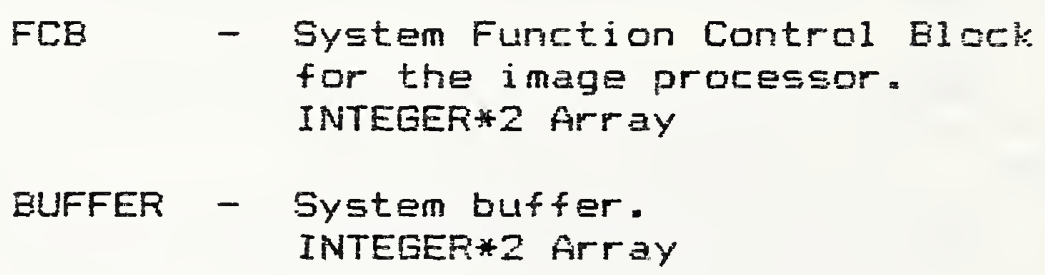

GTCURS cails the following subroutines:

DCLRS

DEXEC

ONCUR

REUTN

The calling sequences for the system supplied subroutines or functions required by each of the major user subroutines are given in Appendix B. These are unique to the host and image processor systems used and ere not transportabie. In order to implement this code on another system, these system cails must be emulated or the entire code converted to any new system calls. 


\subsubsection{Flow Chart}

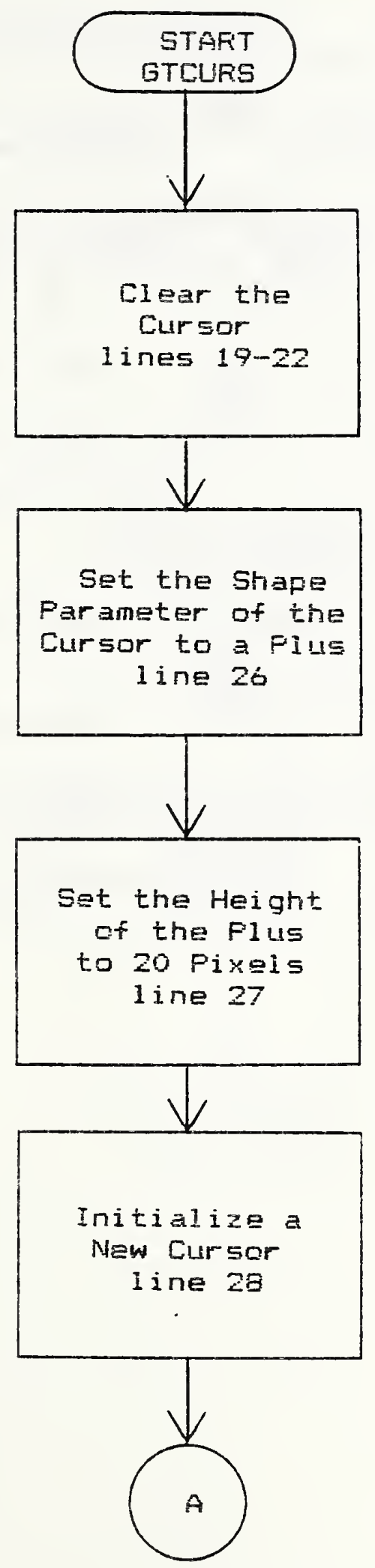




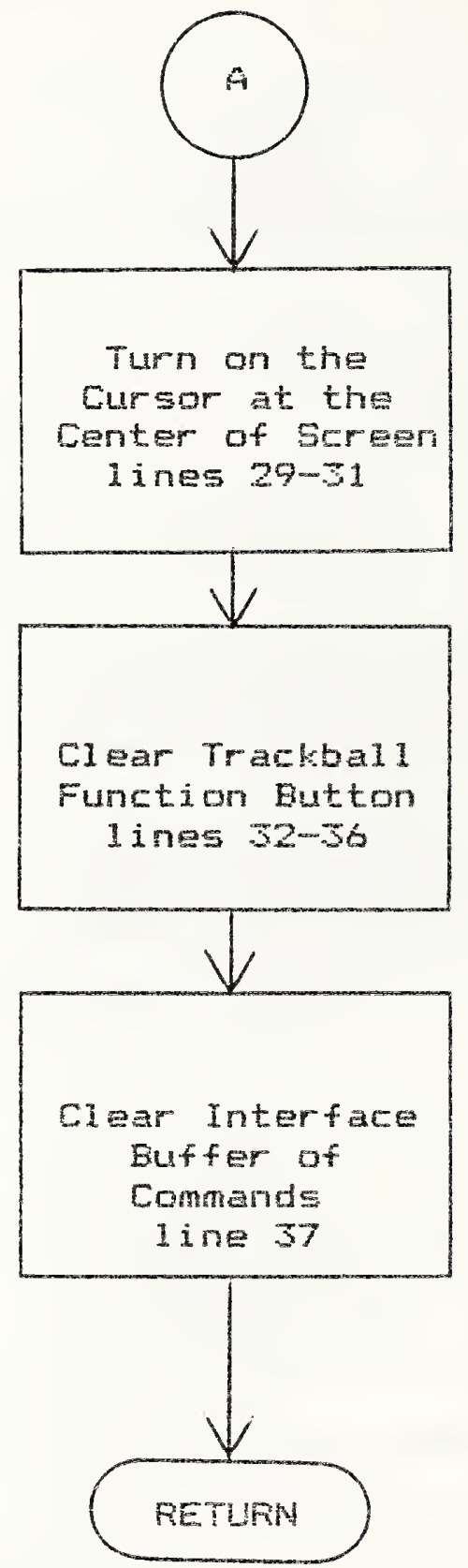


5. 3. 3 Listing

9

SUBROUTINE GTCURS (FCB, BUFR)

10

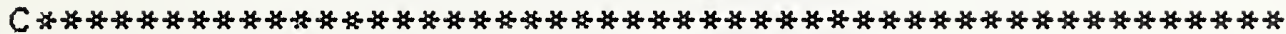
C

C THIS SUBROUTINE INITIALIZES THE CURSOR AT THE

C CENTER POINT OF THE SCREEN

$\mathrm{C}$

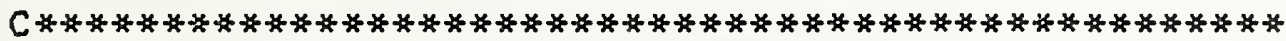

INTEGER*2 FCB $(1), \operatorname{BUFR}(1)$

INTEGER SHAPE

REAL SIZE

C

C CLEAR CURSOR DEFINITION

c

CALL DCURS (FCB, BUFR, 5, 0. O)

$c$

C CREATE a PLUS SHAPED CURSOR

C

SHAPE $=3$

$S I Z E=20$.

CALL DCURS (FCB, BUFR, SHAPE, SIZE)

$I X=255$

$I Y=255$

CALL ONCUR (FCB, BUFR, 1., 0., O., IX, IY, O)

31

320

33 C CLEAR BUTTONS WITH A READ OF BUTTON WORD WHICH IS

34 C O FOR NO BUTTONS PUSHED

$35 \mathrm{C}$

3́

37

CALL RBUTN(FCB, BUTTON, IX, IY)

38

CALL DEXEC (FCB)

RETURN

39 END 
5.4 .1 Summary

This subroutine sets the color specifications for the image processor graphics memory bitplanes. Its calling sequence is:

$$
\text { CALL SETCOL (FCE, BUFFER) : }
$$

The paraneters passed are:

$$
\begin{aligned}
\text { FCB - } & \text { System Function control Biocic } \\
& \text { for the image processor. } \\
& \text { INTEGER*2 Array } \\
\text { EUFFER - } & \text { System buffer array } \\
& \text { INTEGER*Z firray }
\end{aligned}
$$

SETCDL calis the following subroutines:

BCHAN
DEXEC
STCOL
XCOLF


5.4 .2 Flow Chart

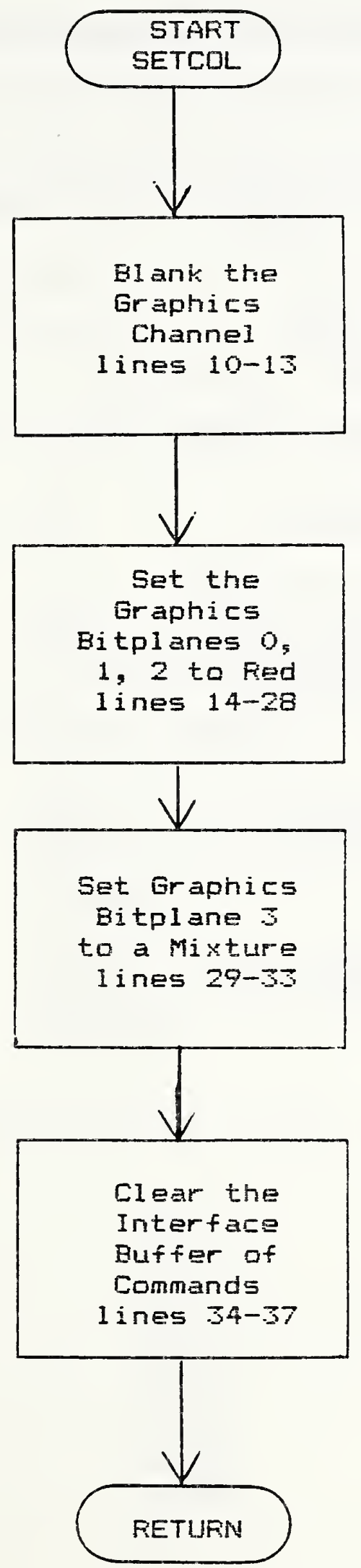


SUBROUTINE SETCOL (FCB, BUFR)

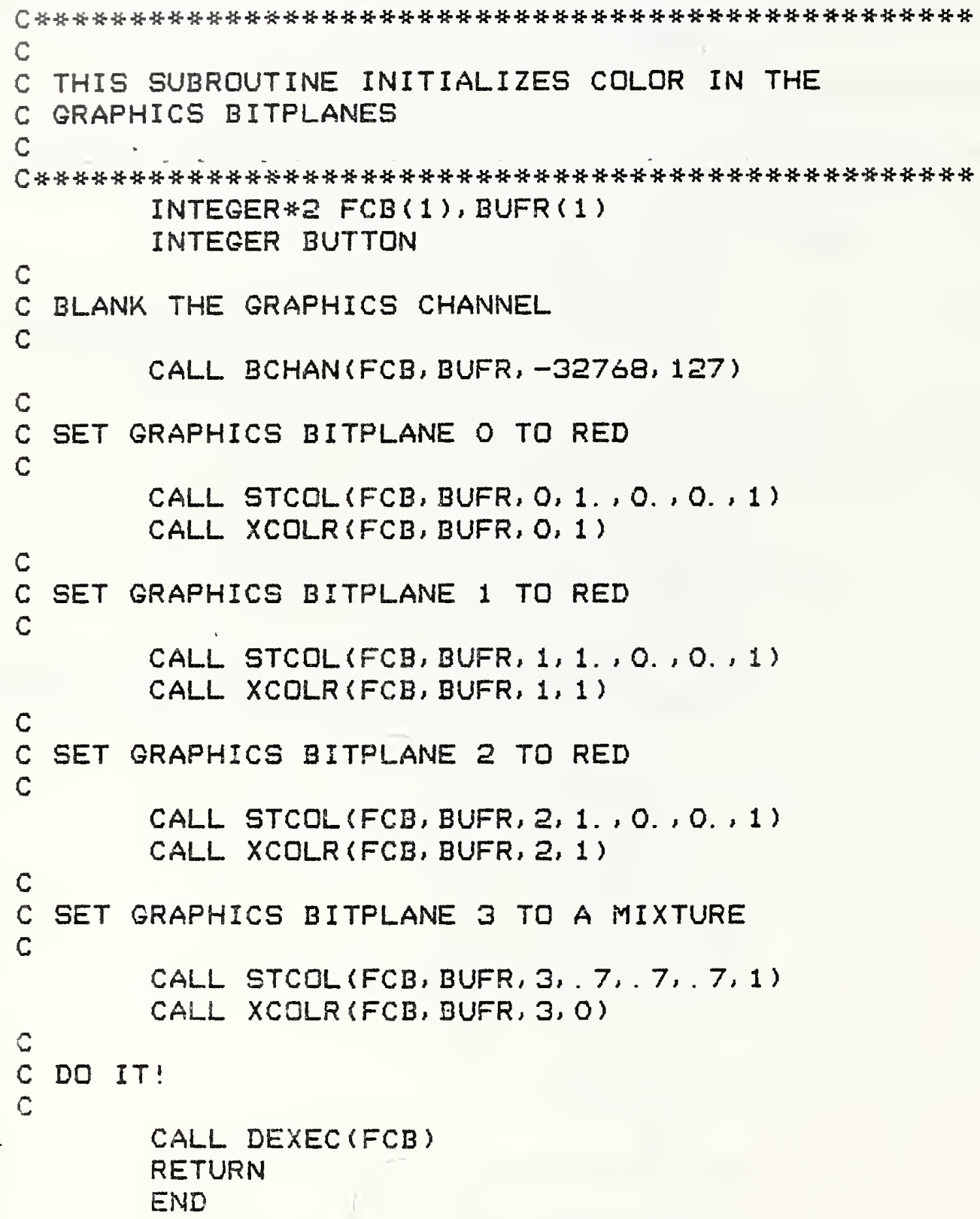


5.5 Subrautine GETFIL

\section{5 .1 Summary}

This subroutine interactively inquires of the user the name of a desired picture file, opens the file, initializes a user selected refresh memory and writes the data file from the host computer to the selected refresh menory in the image processor.

The program assumes that files are formatted as sequential files with 512 records of 512 bytes each. The calling sequence is:

CALL GETFIL (FCB, BUFFER, TAELE, CHANLS).

The parameters passed are:

$\begin{aligned} \text { FCB - } & \text { System Function Control Biock } \\ & \text { for the image processor. } \\ & \text { INTEGER*2 Array } \\ \text { BUFFER - } & \text { System buffer. } \\ & \text { INTEGER*2 Array } \\ \text { TAELE - } & \text { Refresh memory number into } \\ & \text { which to write an image. } \\ & \text { Can be } 1,2, \text { or } 3 . \\ & \text { INTEGER*2 } \\ \text { CHANLS - System channei mask for the } & \text { Selected refresh memory in TABLE. } \\ & \text { INTEGER*2 }\end{aligned}$

GETFIL calis the following subroutines or functions:

SVC7
ZBUFF
INFCE
MSTCL
DADRS
DUNIT
BCHAN
SYSID
IMAGE
DMASK




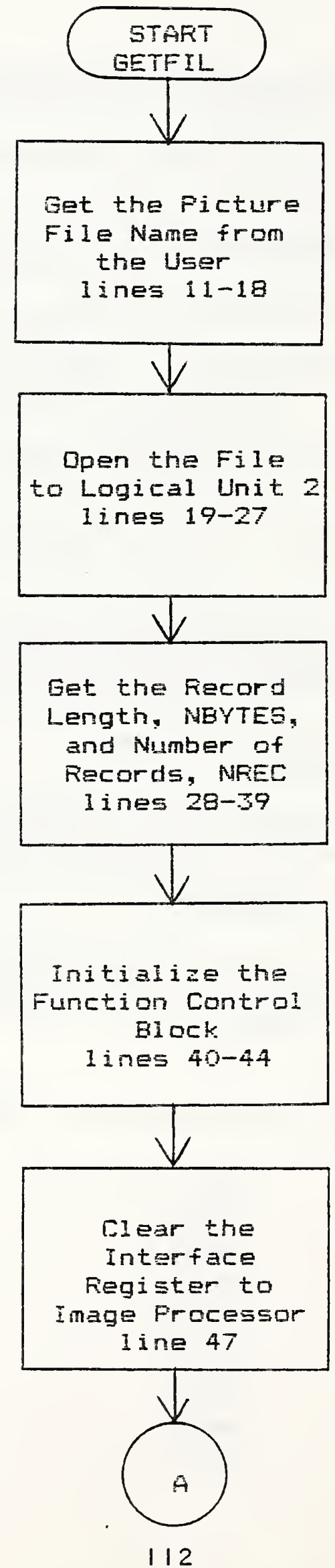




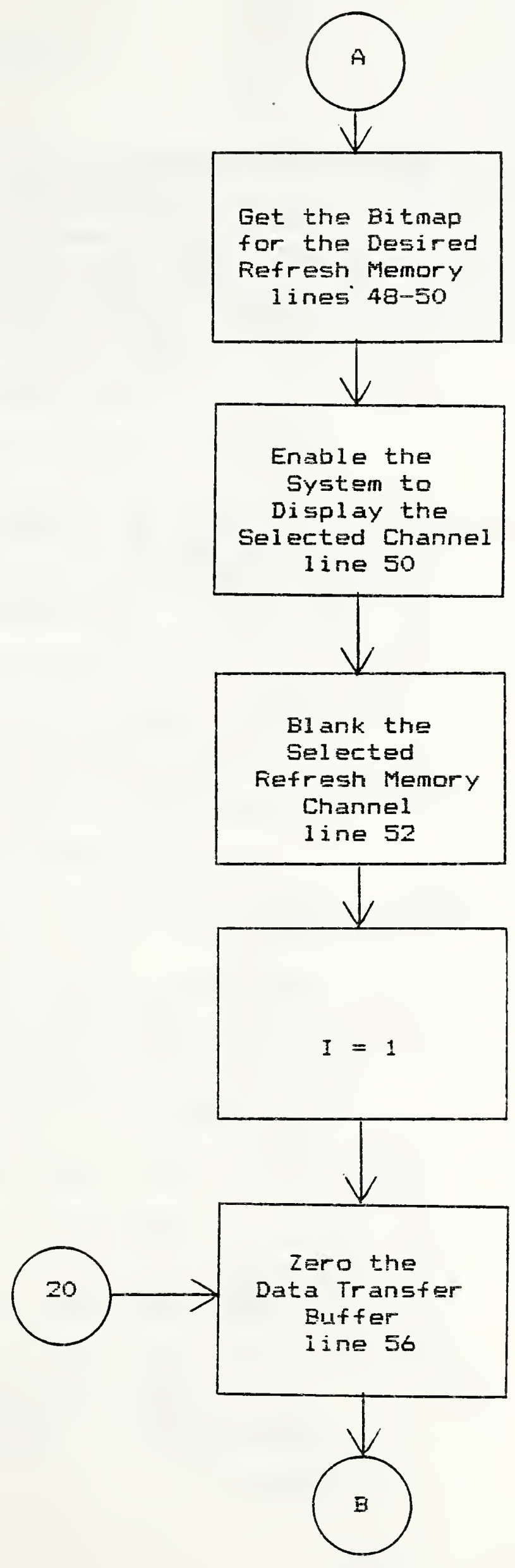




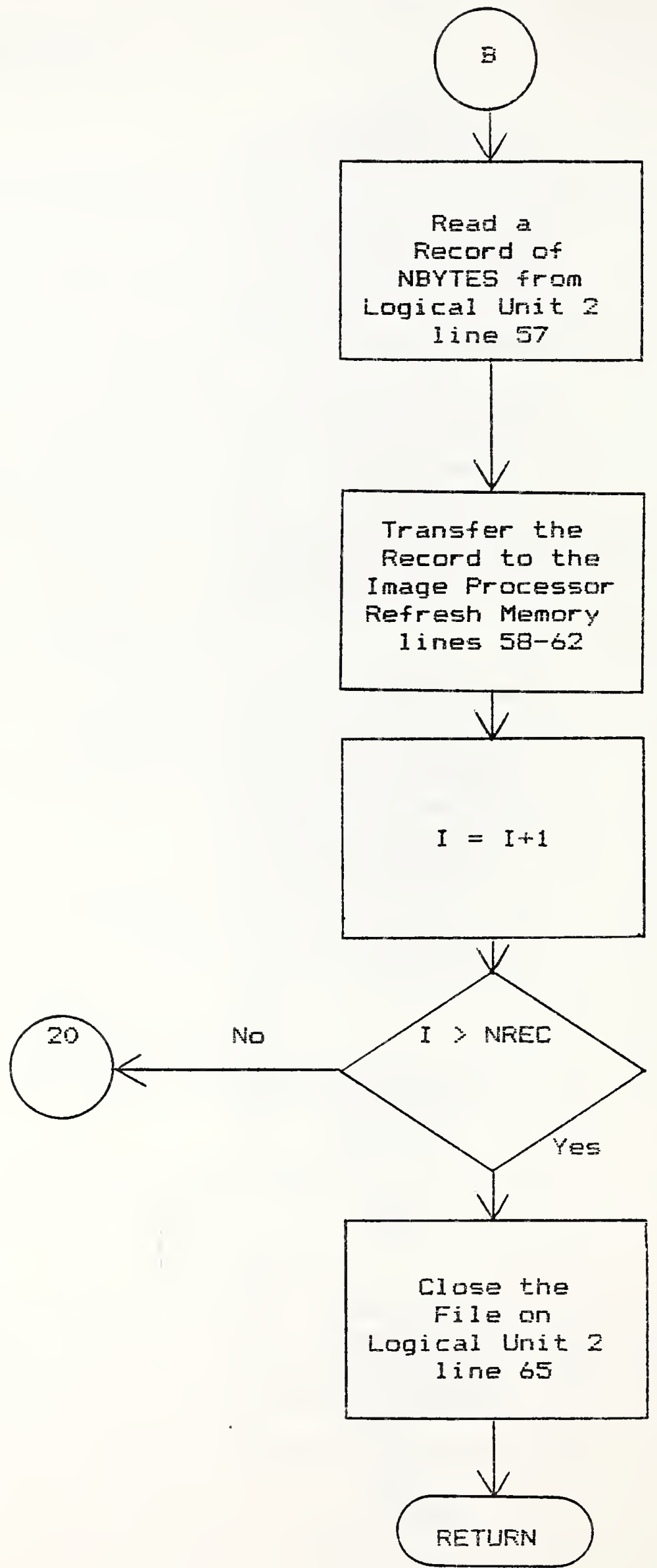




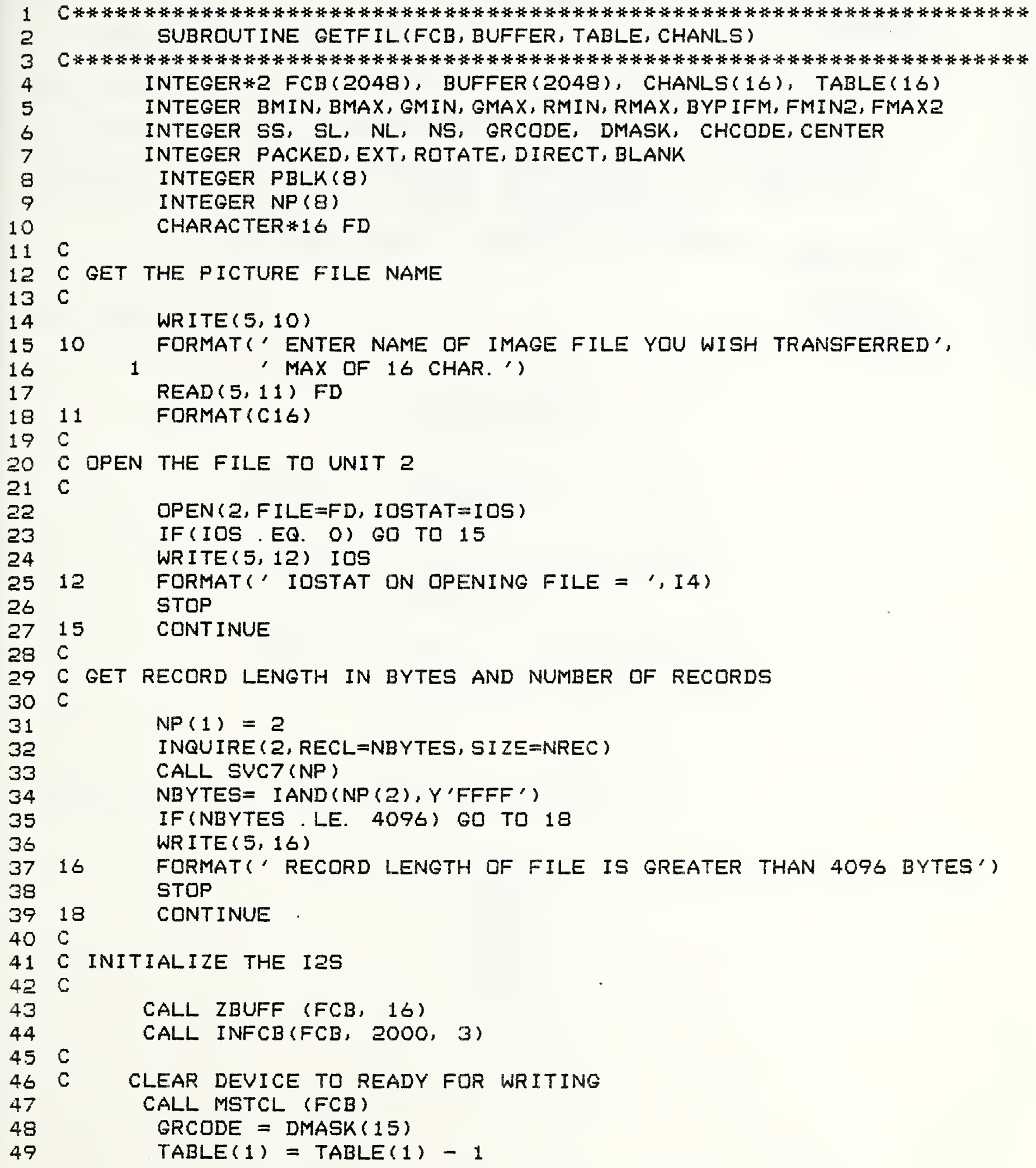




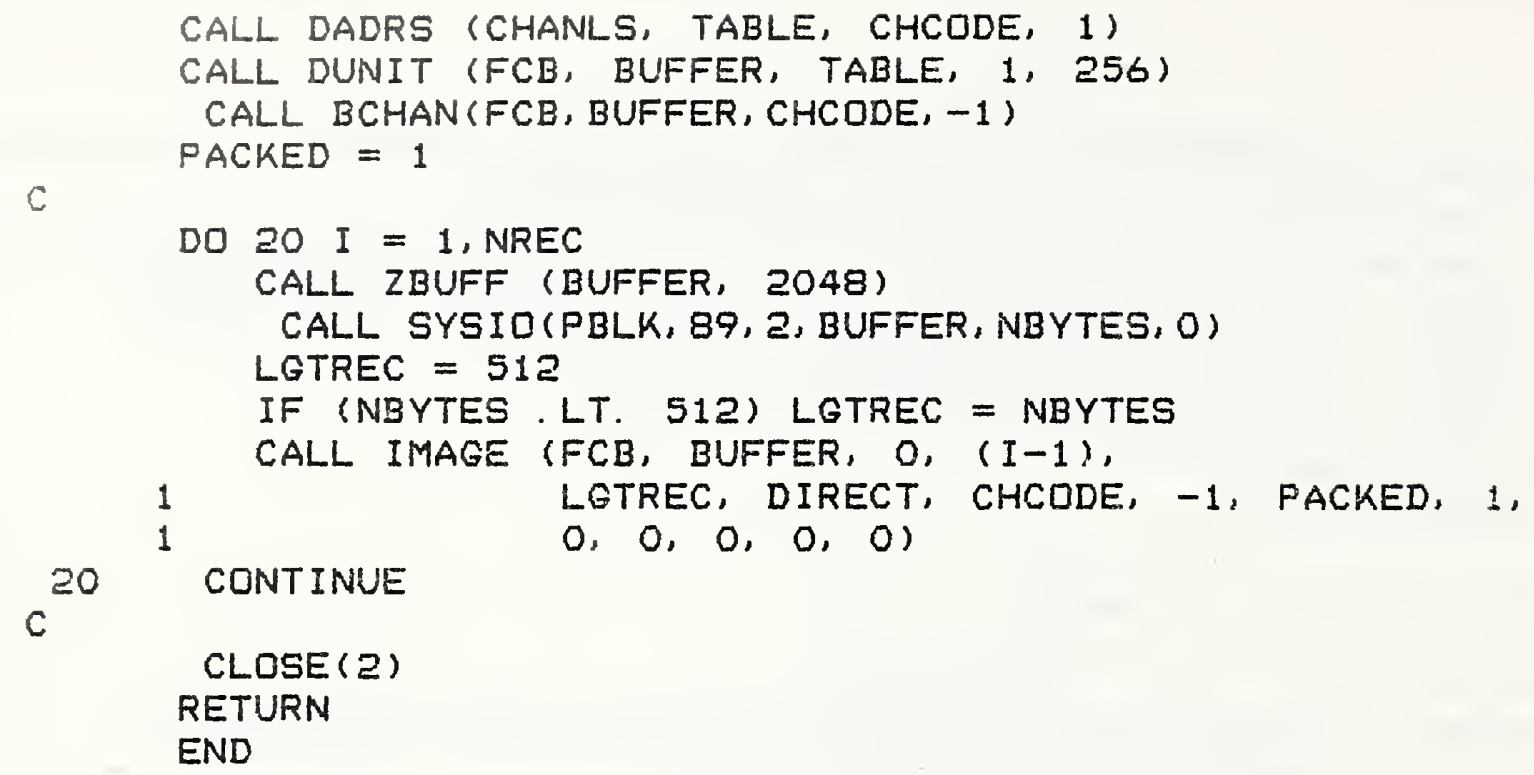


5.6 Subroutine GETCHN

5.6.1 Summary

This subroutine initializes a specified channel and enables the registers for that selected channel so that an image may be displayed. No image is actually transferred. The calling sequence for this subroutine is:

CALL GETCHN (FCE, BUFFER, TABLE, CHANLS).

The parameters passed are:

$$
\begin{aligned}
& \text { FCB - System Function Control } \\
& \text { Block array. } \\
& \text { INTEGER * } 2 \text { Array } \\
& \text { BUfFER - System buffer array. } \\
& \text { INTEGEF* } 2 \\
& \text { TABLE - Refresh memory number. } \\
& \text { Set to } 1,2 \text { or } 3 . \\
& \text { INTEGEF*2 } \\
& \text { CHANLS - Channel mask for the } \\
& \text { refresh memory in TABLE. } \\
& \text { INTEGER*2 }
\end{aligned}
$$

GETCHN cails the following subroutines:

ZBUJFF

INFCB

MGTCL

DADRS

DUNIT

BCHAN 


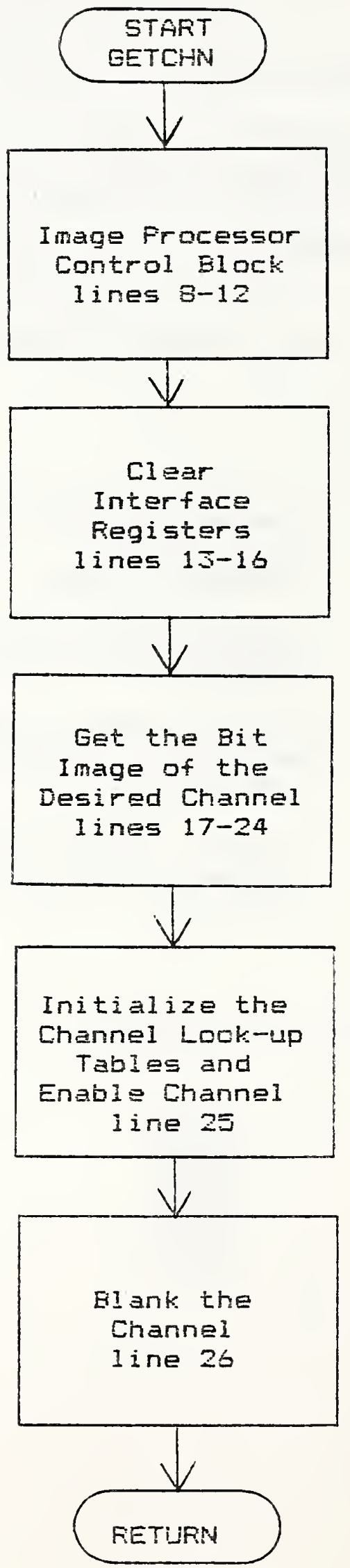


5.6 .3 Listing

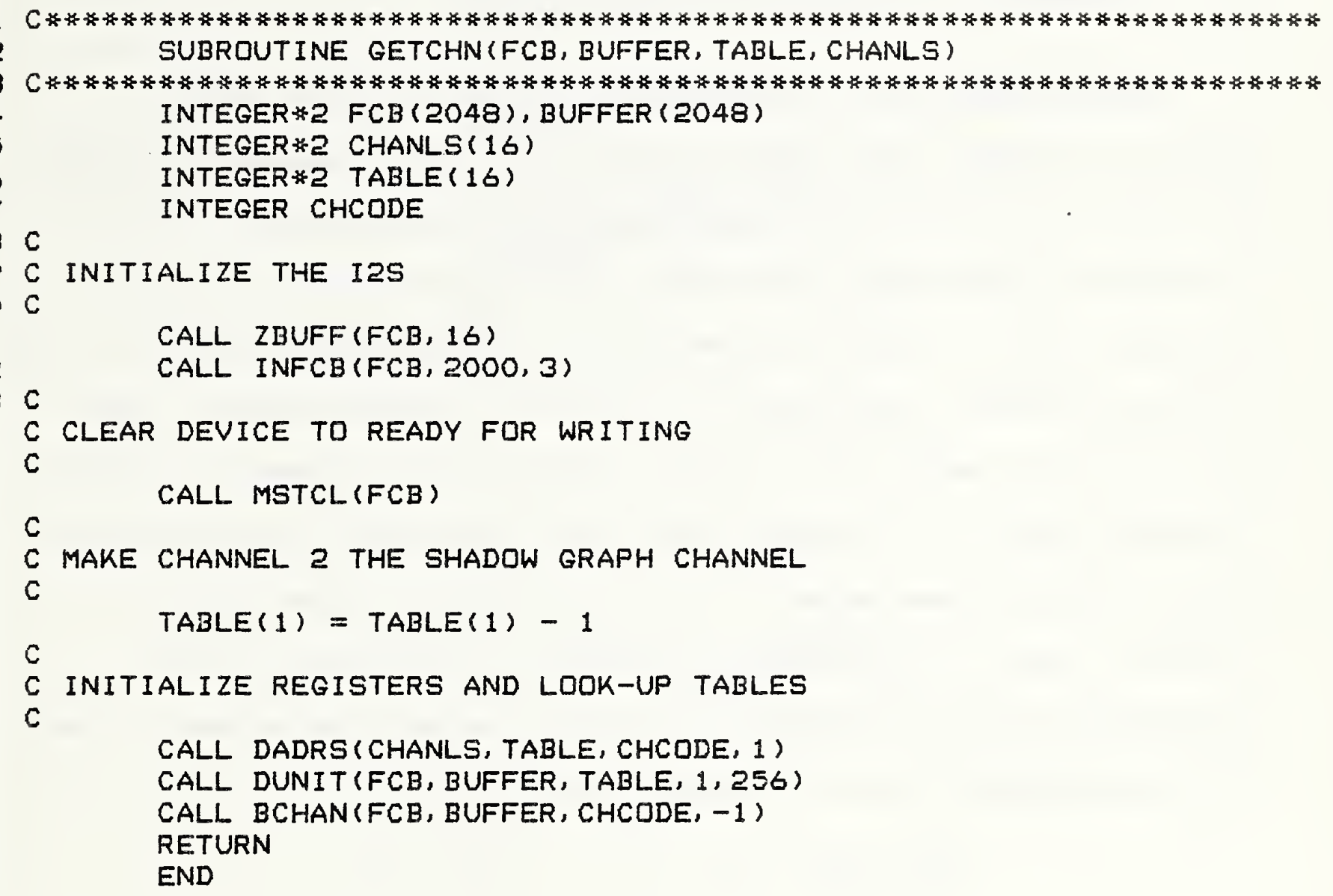




\section{7 .1 Summary}

Given a point in a unit square in the $X Y-p$ lane and a direction vector (W(1), W(2)), this subroutine determines whether the point is interior to the square or on the boundary. If it is interior to the square, then the subroutine peturns the exit boundary point of the directed line through the point with direction vector $(W(1), W(2))$. If it is a boundary point, tren the direction vector (W(1), W(2)) either points inward or outward from the square. If inward, then the subroutine returne the exit point from the same square. If the direction vector points outward, then the subroutine returns the exit point of the neighboring square through which the directed line passes. The calling sequence for this subroutine is

CALL GNXY $\left(X_{*}, Y, I X, I Y, W_{*}\right.$ IFLG).

GNXY passas the following parameters:

\begin{tabular}{|c|c|c|}
\hline ON INFUT & - & \\
\hline$X, Y$ & - & $\begin{array}{l}\text { Components of the point of interest. } \\
\text { REAL }\end{array}$ \\
\hline$I X, I Y$ & - & $\begin{array}{l}\text { Truncated values ot } x, y \text { respectively. } \\
\text { INTEGER }\end{array}$ \\
\hline$W(1), w(2)$ & - & $\begin{array}{l}X, Y \text { components of the } 3-D \text { direction } \\
\text { Vector } \vec{H} \text {. } \\
\text { REAL }\end{array}$ \\
\hline
\end{tabular}


ON DUTPUT

$$
\begin{array}{ll}
X, Y & X, Y \text { components of exit point for the } \\
& \text { unit square of adjacent unit square. } \\
& \text { REAL } \\
\text { IX, IY } & \text { Truncated values of } X, Y \text { respectively. } \\
& \text { INTEGEF } \\
\text { IFLG } & =1 \text { if W(1) }=W(Z)=0 \\
& =0 \text { otherwise. } \\
& \text { INTEGER }
\end{array}
$$

No subroutines are called. 


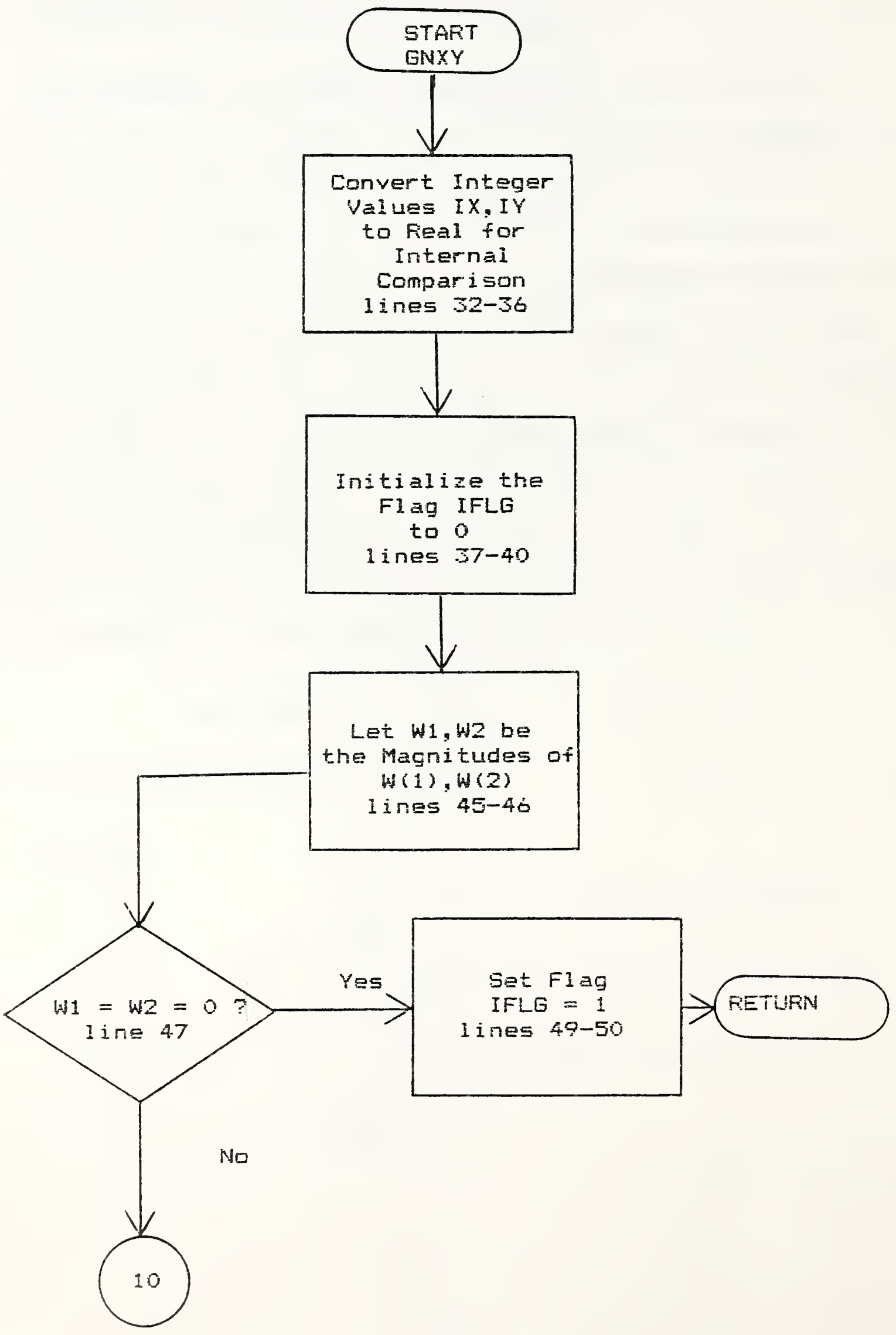




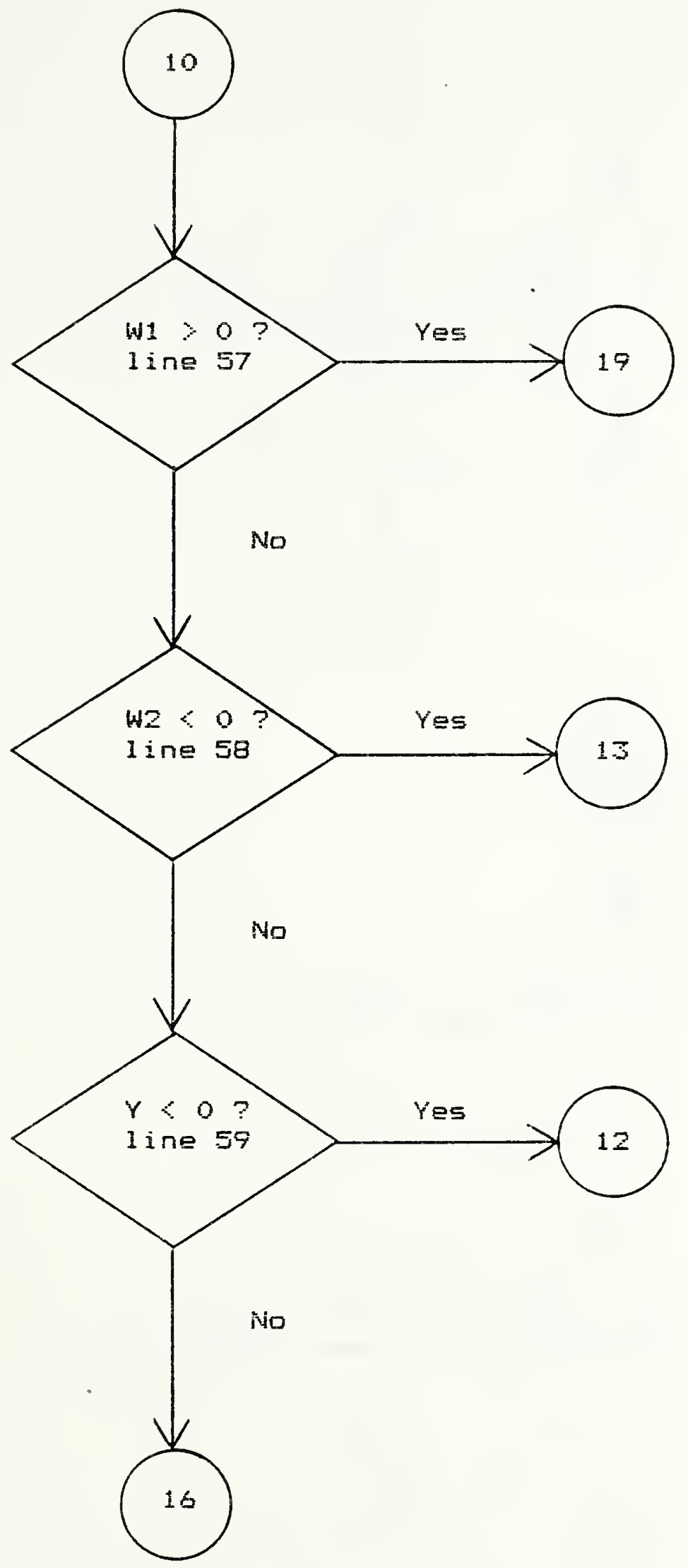



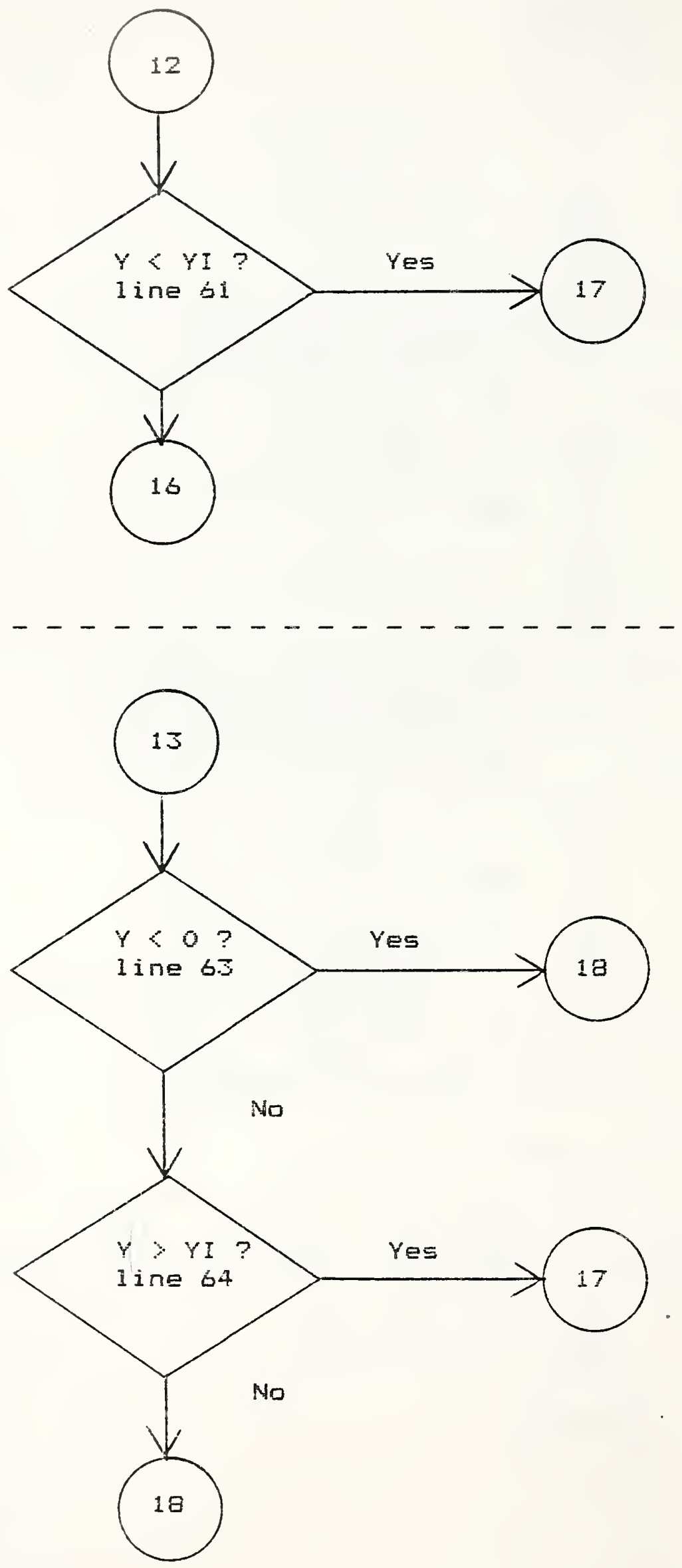

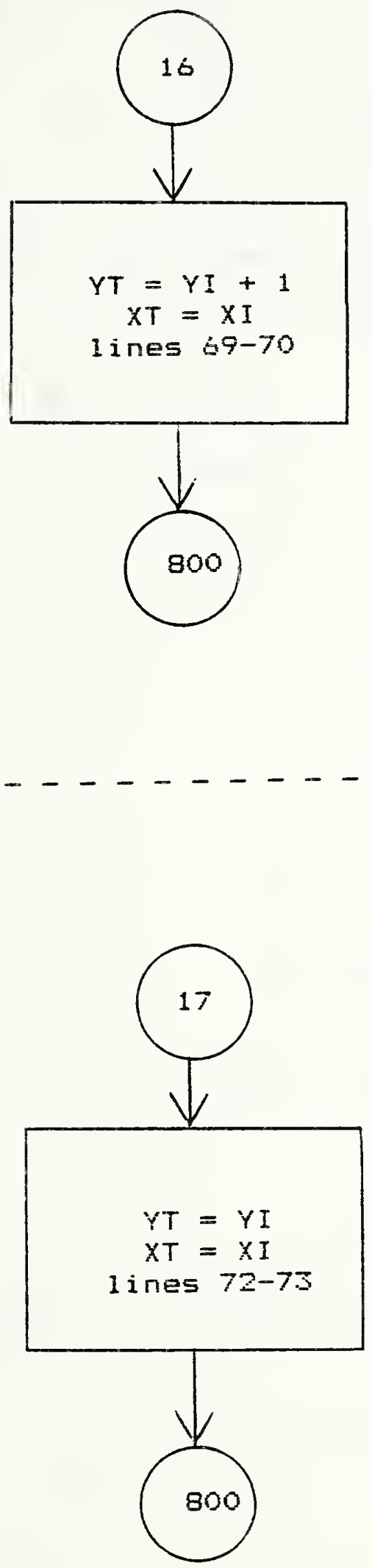


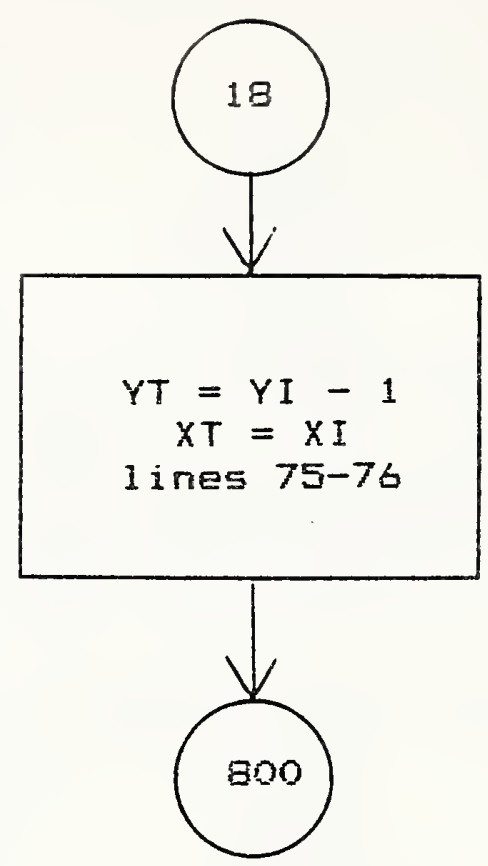

$----\ldots-\ldots-\ldots-\cdots-\cdots-\cdots$

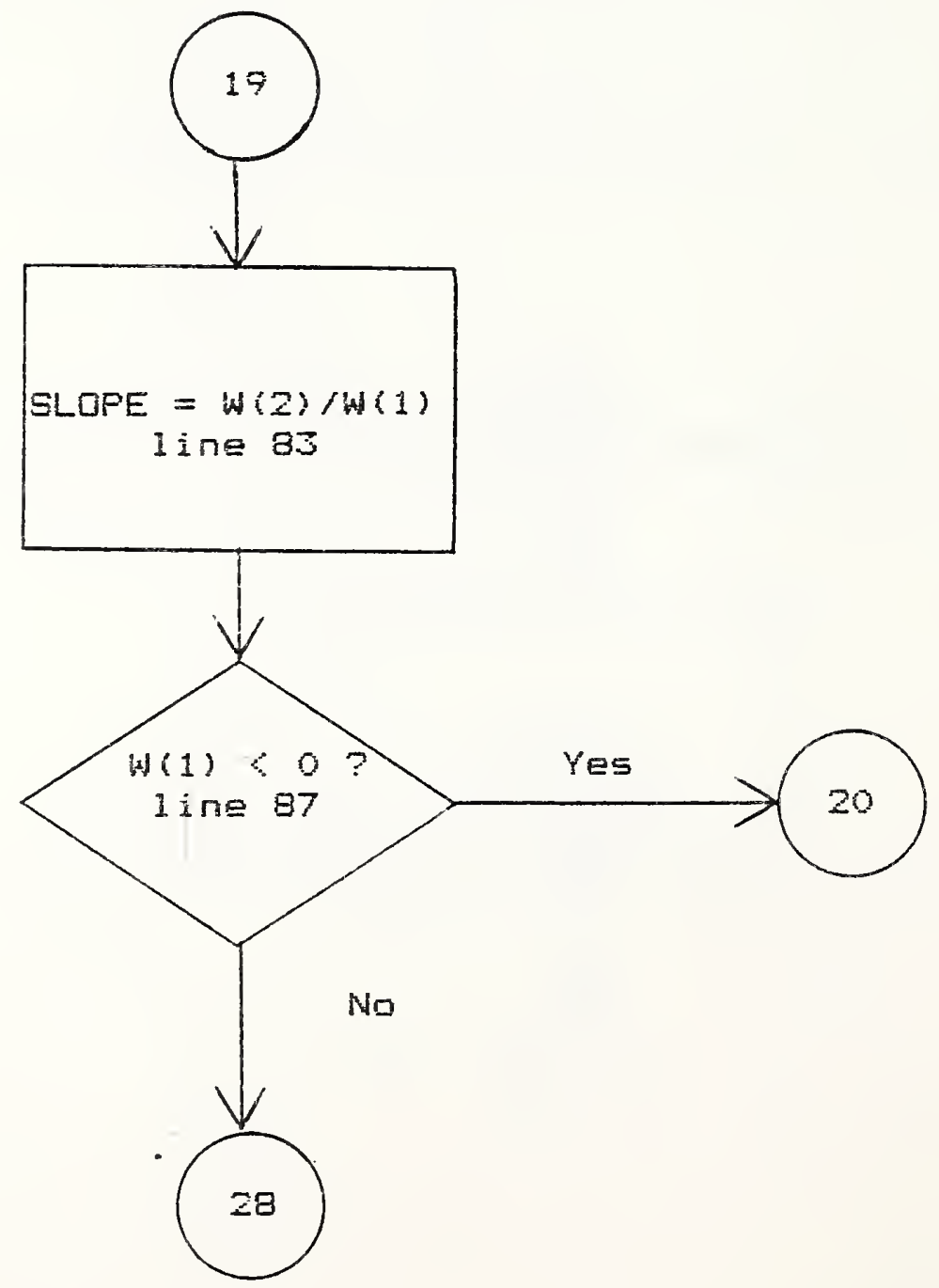



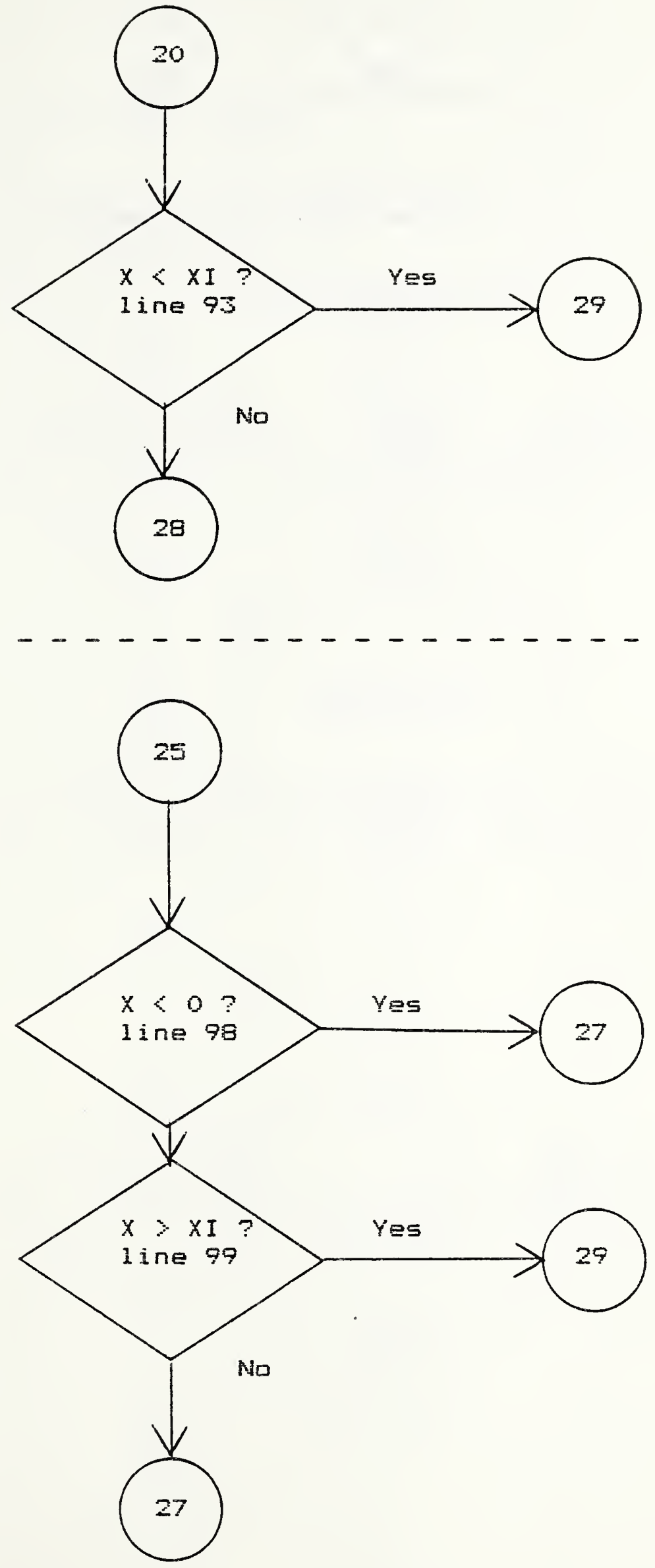


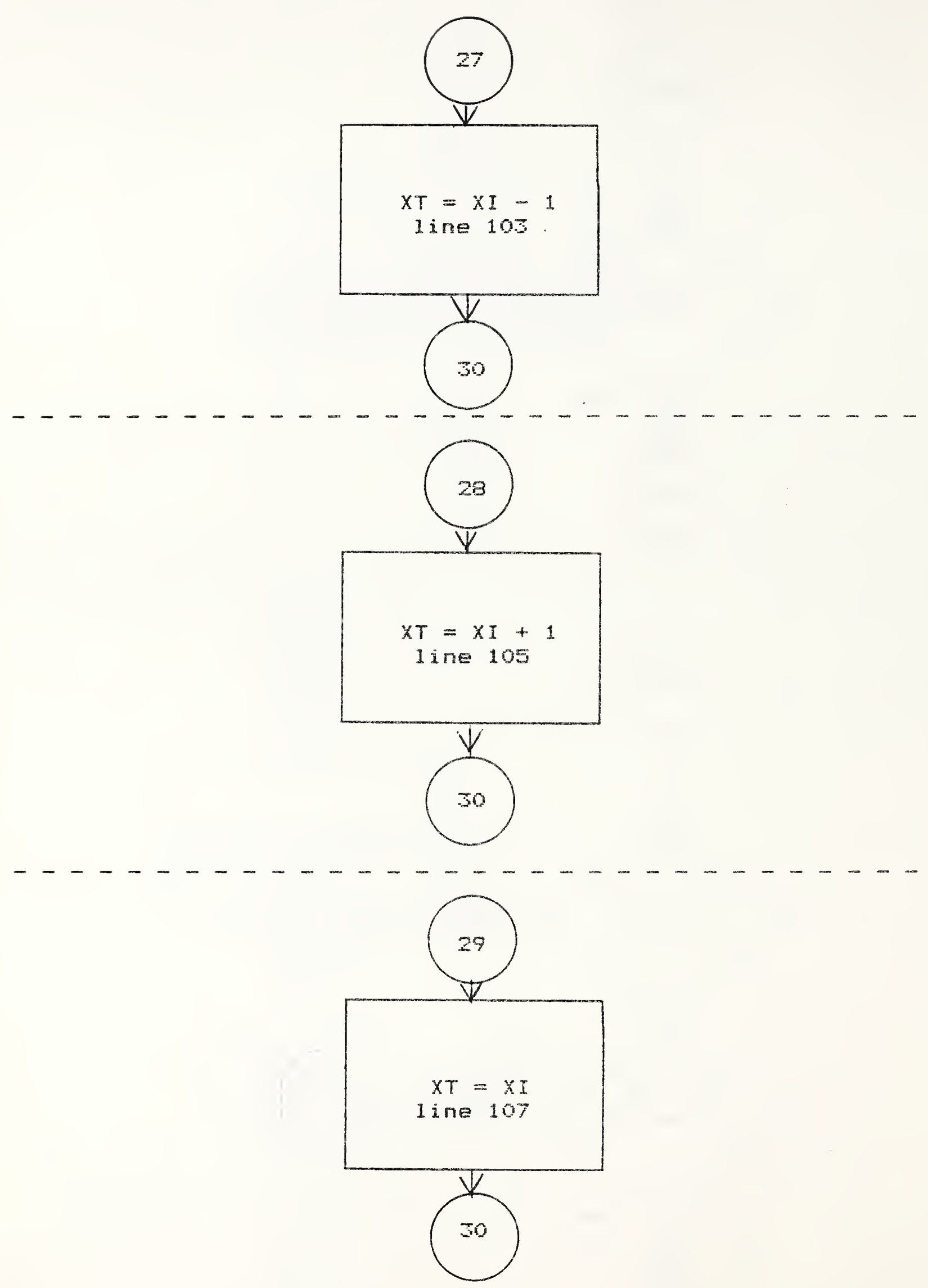




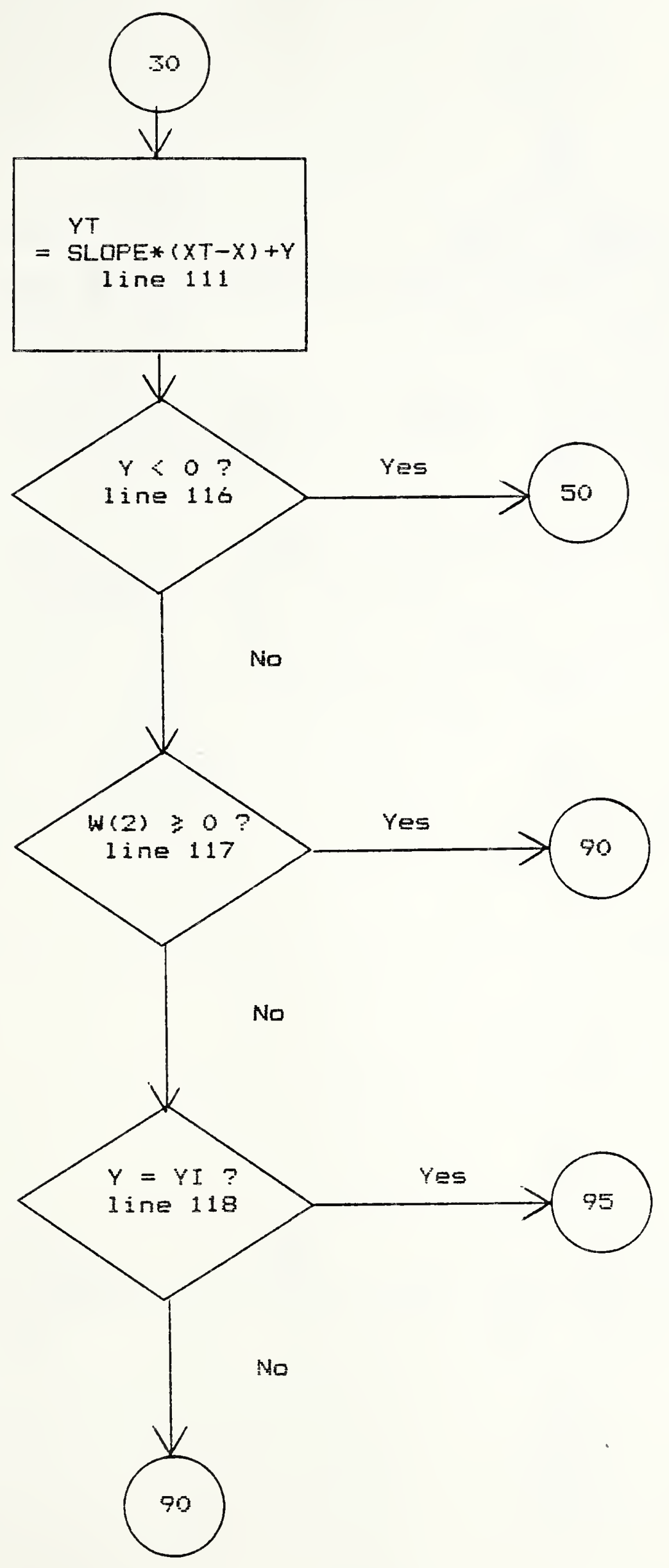



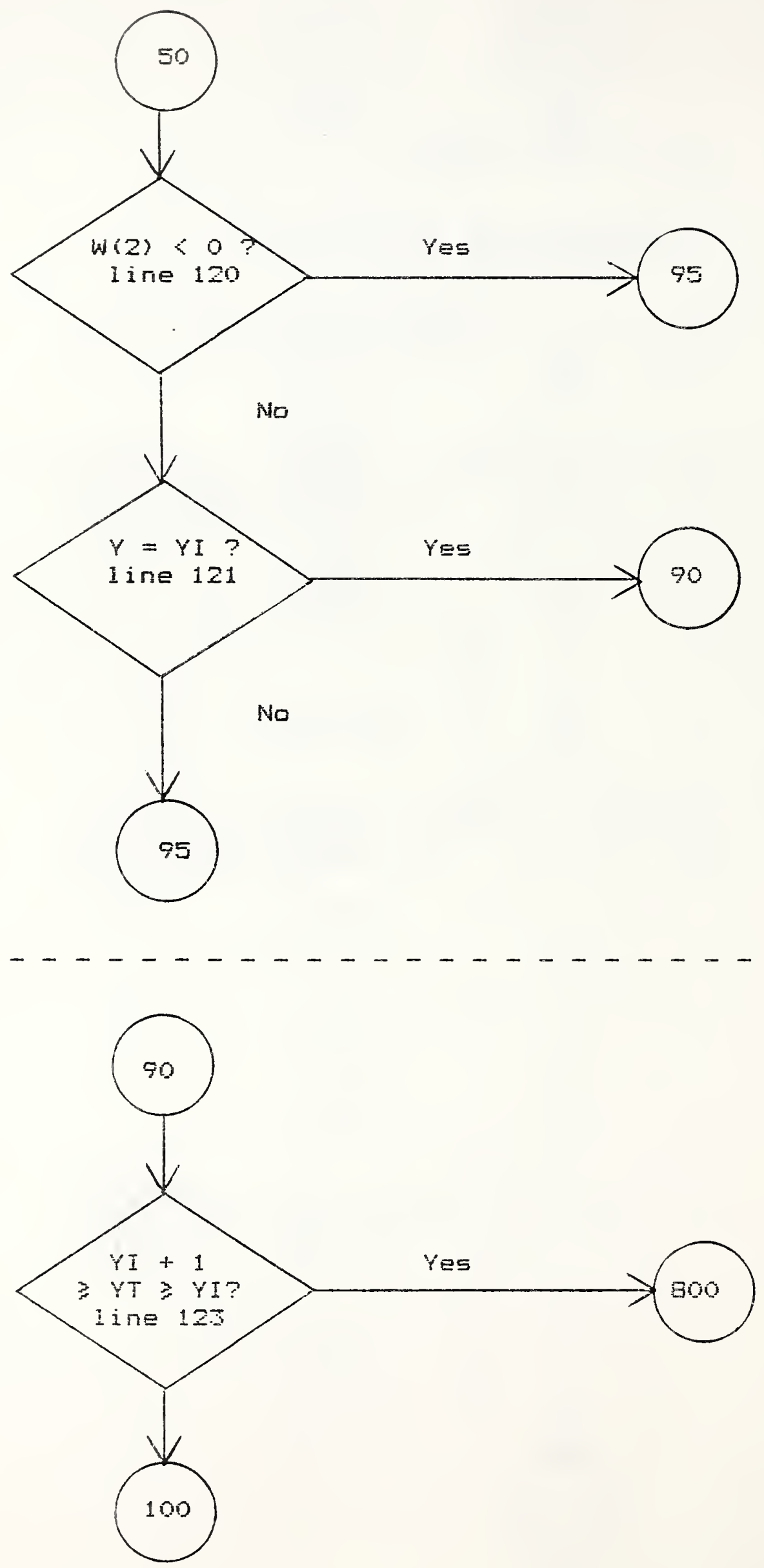


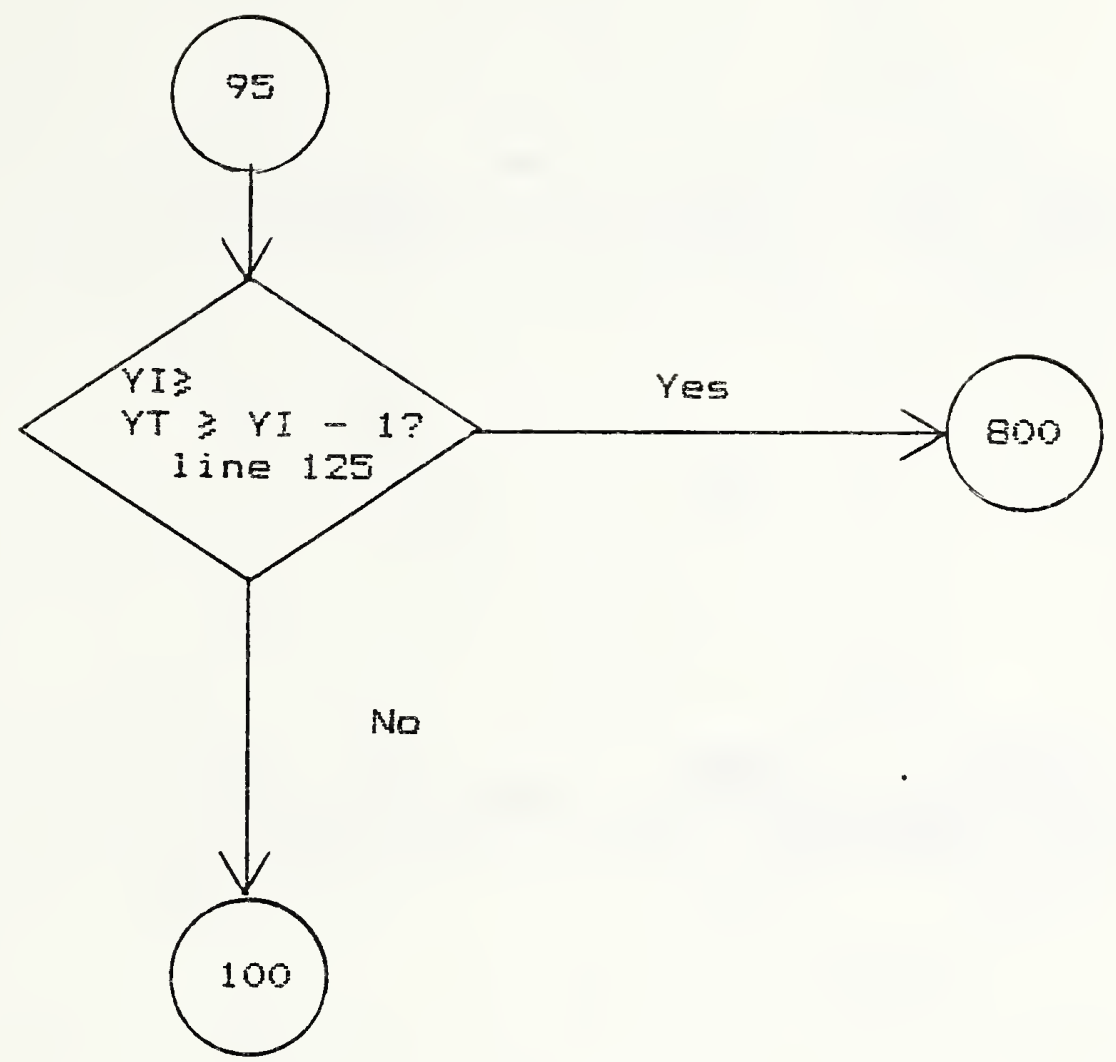




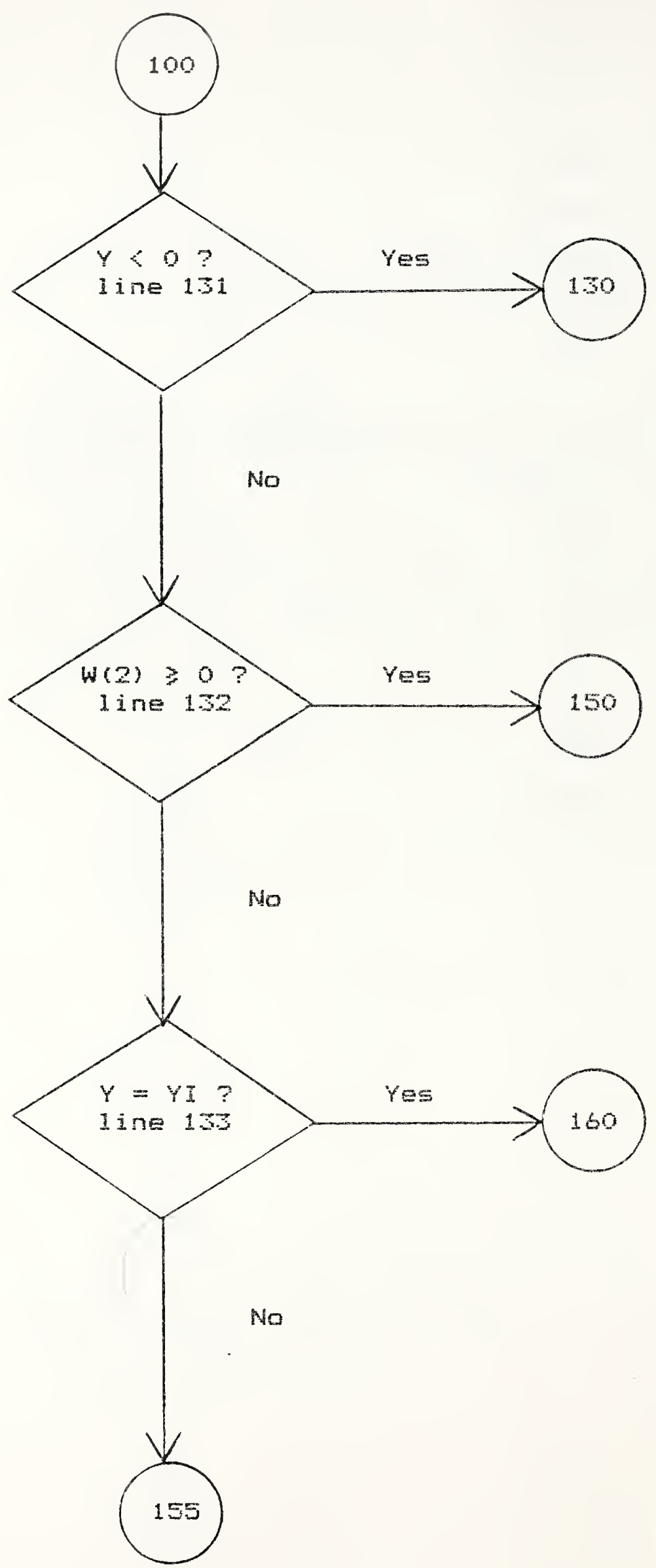




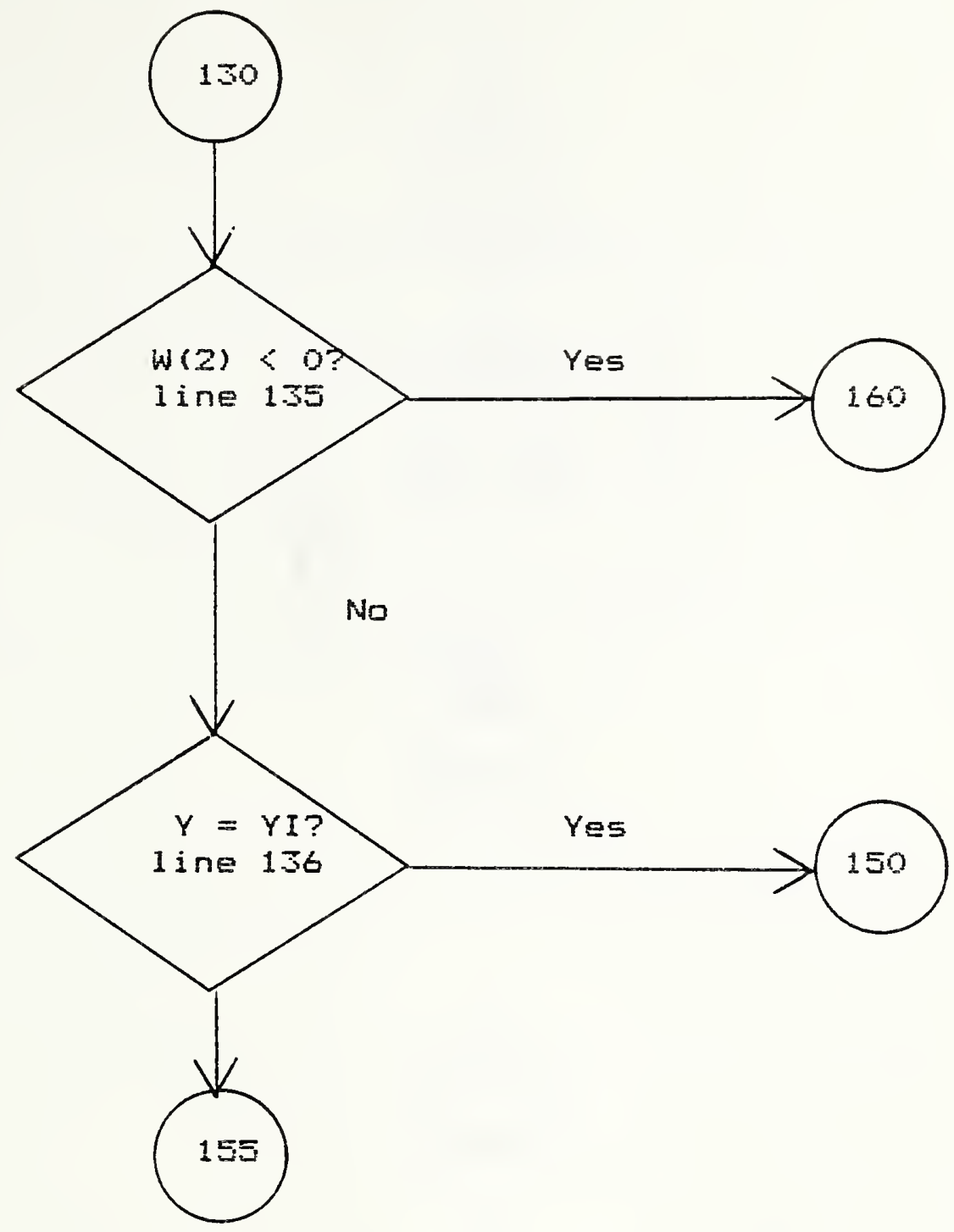

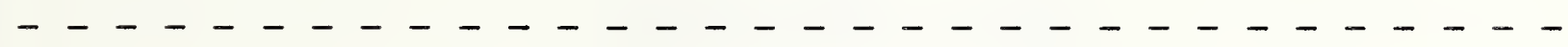

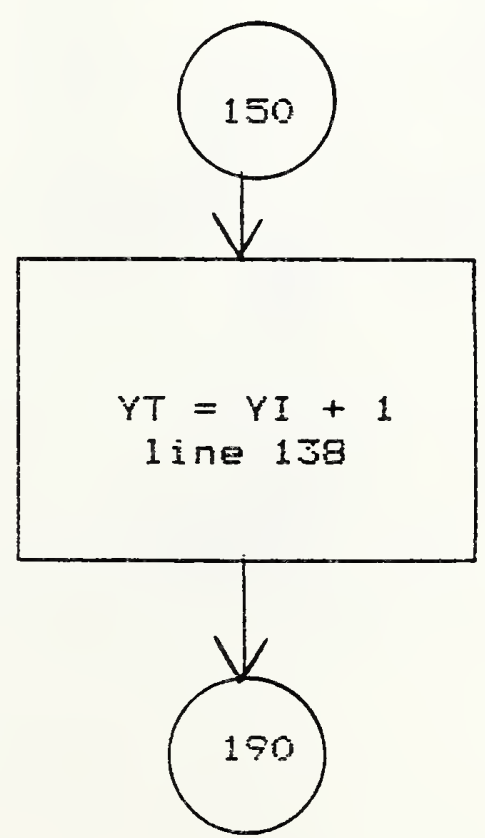




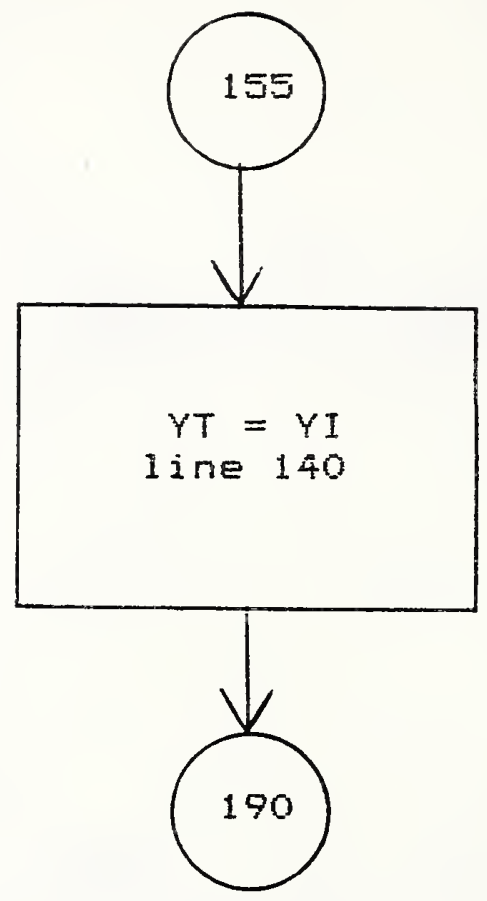

-

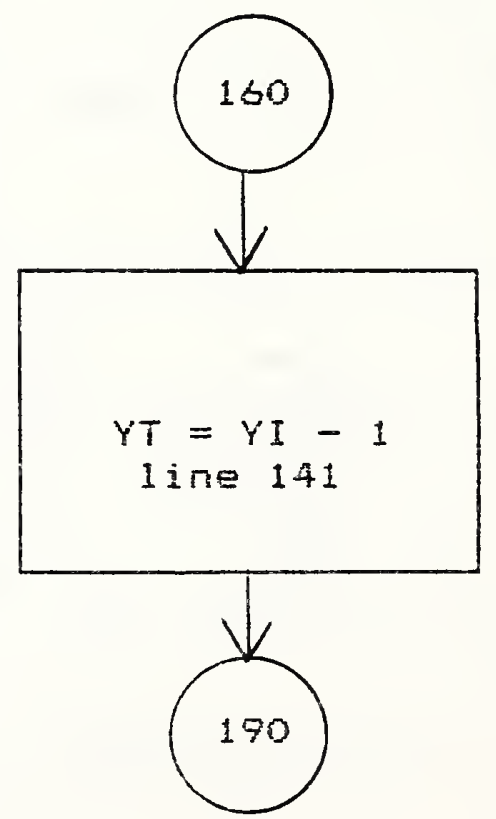




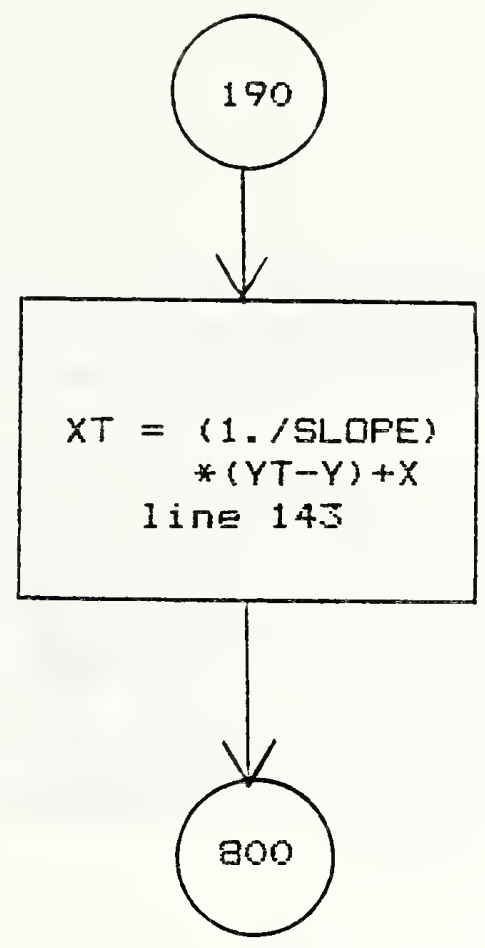

$-\ldots-\ldots-\ldots-\ldots-\ldots-\cdots$

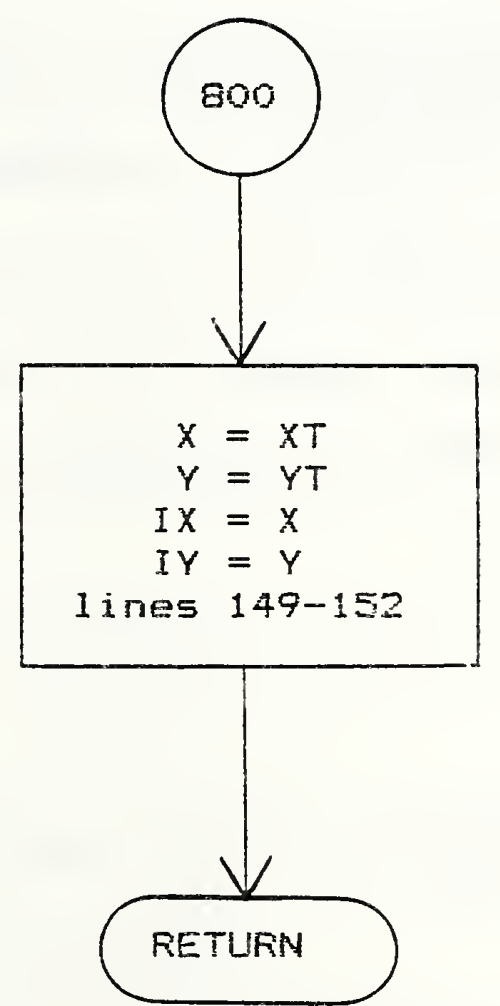


1

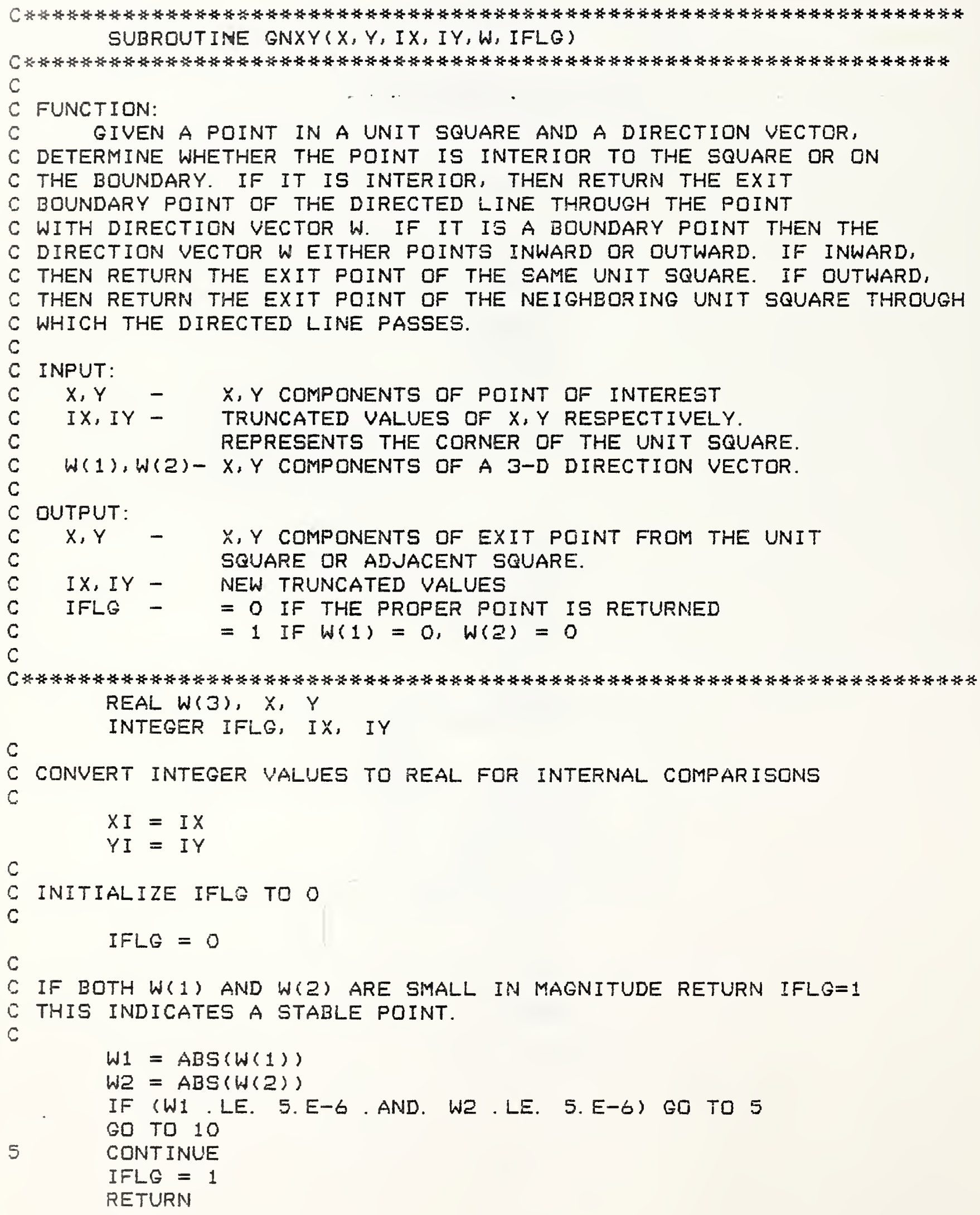




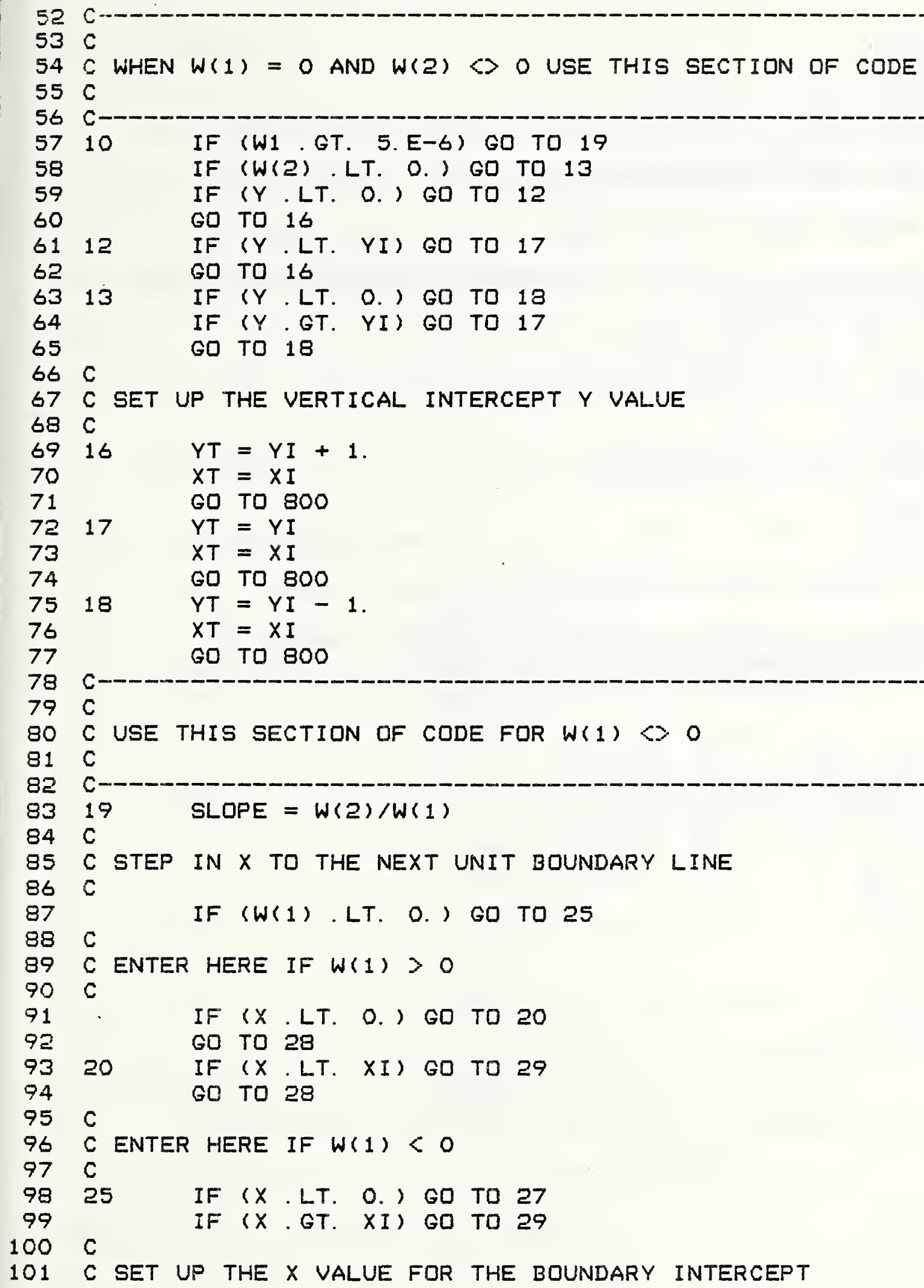




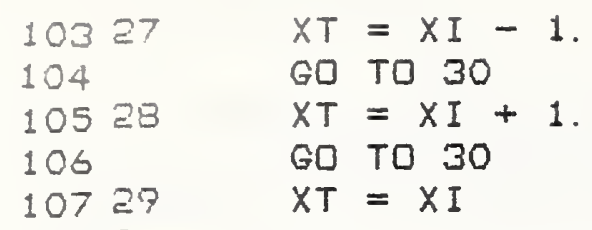

152

153

154

$$
\begin{aligned}
& X=X T \\
& Y=Y T \\
& I X=X \\
& I Y=Y \\
& \text { RETURN } \\
& \text { END }
\end{aligned}
$$


5.8. 1 Summary

This subroutine transfers NPIXEL number of pixels to the image processor channel refresh memory, with bitmap channel number in FILE(3), beginning in IFOW row and ICOL column and proceeding to the right. The error flag is not used in this version. The data is transferred through the array BuF with one pixel per word. The calling sequence is:

CALL WRPIC (FCB, FILE, BUF, IFOW, ICOL, NPIXEL, IERF).

The parameters passed are:

\begin{tabular}{|c|c|c|}
\hline FCB & - & $\begin{array}{l}\text { System Function Contral Block. } \\
\text { INTEGER* } 2 \text { Array }\end{array}$ \\
\hline FILE & - & $\begin{array}{l}\text { Array containing the bitmap for } \\
\text { the desired refresh memory in } \\
\text { el ement } 3 . \\
\text { INTEGER Array }\end{array}$ \\
\hline EUF & - & $\begin{array}{l}\text { A buffer array that contains the } \\
\text { transferred pixel data one pixel } \\
\text { per word. } \\
\text { INTEGER*? Array }\end{array}$ \\
\hline IROW & - & $\begin{array}{l}\text { Row index from } 0 \text { to } 511= \\
\text { INTEGER }\end{array}$ \\
\hline ICOL & - & $\begin{array}{l}\text { Column index from o to } 511 \text {. } \\
\text { INTEGER }\end{array}$ \\
\hline NPIXEL & - & $\begin{array}{l}\text { Number of pixels to transfer. } \\
\text { INTEGER }\end{array}$ \\
\hline IEAR & - & Error flag. Not used. \\
\hline
\end{tabular}

WFPIC calls the subroutine IMAGE. 
5.8.2 Flow Chart

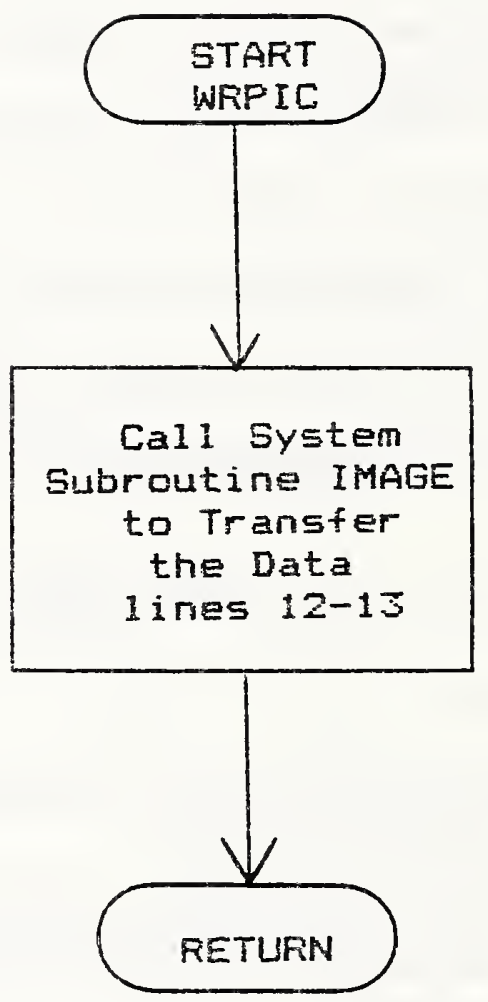


5.8 .3 Listing

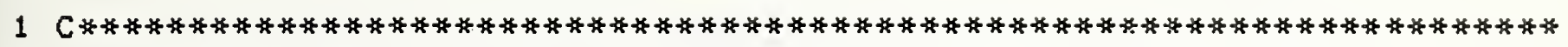
2 SUBROUT INE WRPIC (FCB, FILE, BUF, IROW, ICOL, NP I XEL, IERR)

3 C $3 *$ * 4 INTEGER *2 BUF (NPIXEL), FCB (2048)

5 INTEGER FILE (7)

$6 C$

7 C IMAGE METROLDGY WRPIC $7 / 30 / 80$

$8 \mathrm{C}$ WRITES FROM DISPLAY DEVICE

9 C FILE( 3 ) IS CHANNEL NUMBER

$10 \mathrm{C}$ WRITES A ROW FROM LEFT TO RIGHT

$11 \mathrm{C}$

12

13

14

15

16

CALL IMAGE (FCB, BUF, ICOL, IROW, NP IXEL, O, *FILE (3), -1, 0, 1, 0, 0, 0,0,0)

IERR $=0$

RETURN

END 


\subsubsection{Summery}

This subroutine transfers NPIXEL number of pixels from the image processor refresh memory with bitmap in FILE(3) to BUF: one byte per word; beginning in IfDly row and ICOL column. The error flag is not used in this version. The caling sequence for this subroutine is:

CALL FDPIC (FCB, FILE, BUF, IFDW, ICOL, MPIXEL, IERF).

The parameters passed are:

\begin{tabular}{|c|c|c|}
\hline $\mathrm{FCE}$ & - & $\begin{array}{l}\text { System Function Control biock. } \\
\text { INTEGER*2 Array }\end{array}$ \\
\hline FILE & - & $\begin{array}{l}\text { Array that contains the bitmap } \\
\text { for the desired refresh memory } \\
\text { in FILE (3). } \\
\text { INTEGER Array }\end{array}$ \\
\hline BUF & - & $\begin{array}{l}\text { Buffer array that receives the } \\
\text { data from the transfer, one } \\
\text { byte per word. } \\
\text { INTEGER*2 Array }\end{array}$ \\
\hline IFON & - & $\begin{array}{l}\text { Eeginning row numior of the } \\
\text { refresh memory for data transfer } \\
\text { INTEGER }\end{array}$ \\
\hline $\mathrm{ICOL}$ & - & $\begin{array}{l}\text { Beginning calumn number of the } \\
\text { refresh memory for data transfer. } \\
\text { INTEGEF }\end{array}$ \\
\hline NPIXEL & - & $\begin{array}{l}\text { Number of pixels to transfer } \\
\text { INTEGER }\end{array}$ \\
\hline IERA & - & $\begin{array}{l}\text { Error flag. Not used in this } \\
\text { version. }\end{array}$ \\
\hline
\end{tabular}


RDPIC calls the following subroutines:

IMAGE

ISBYTE . 


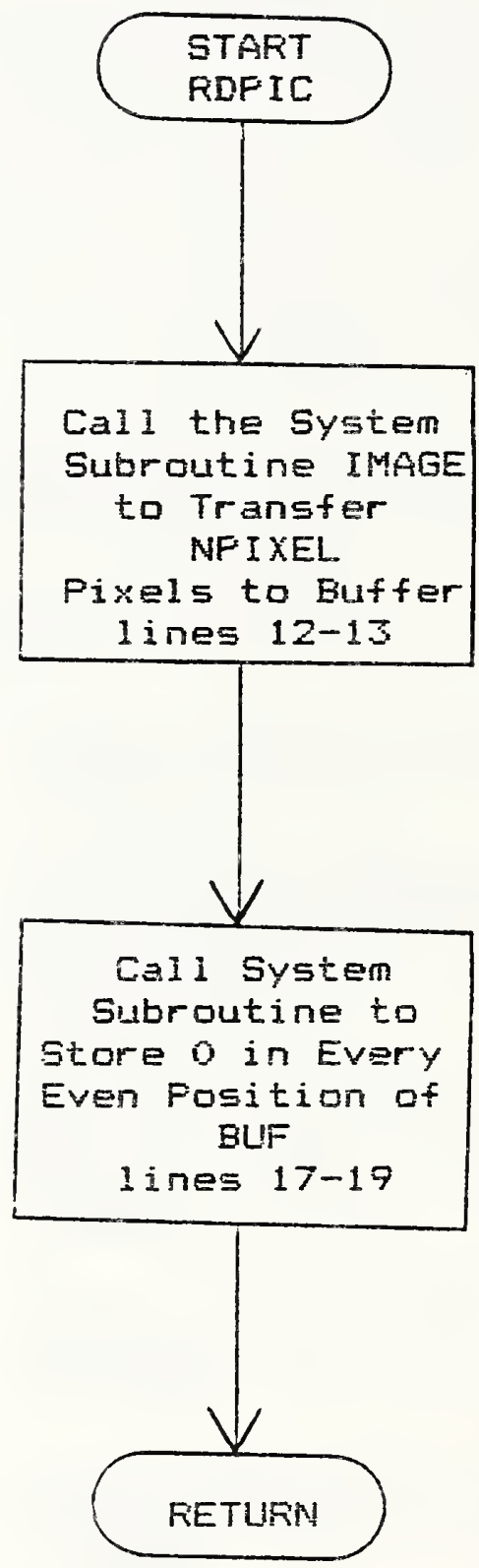




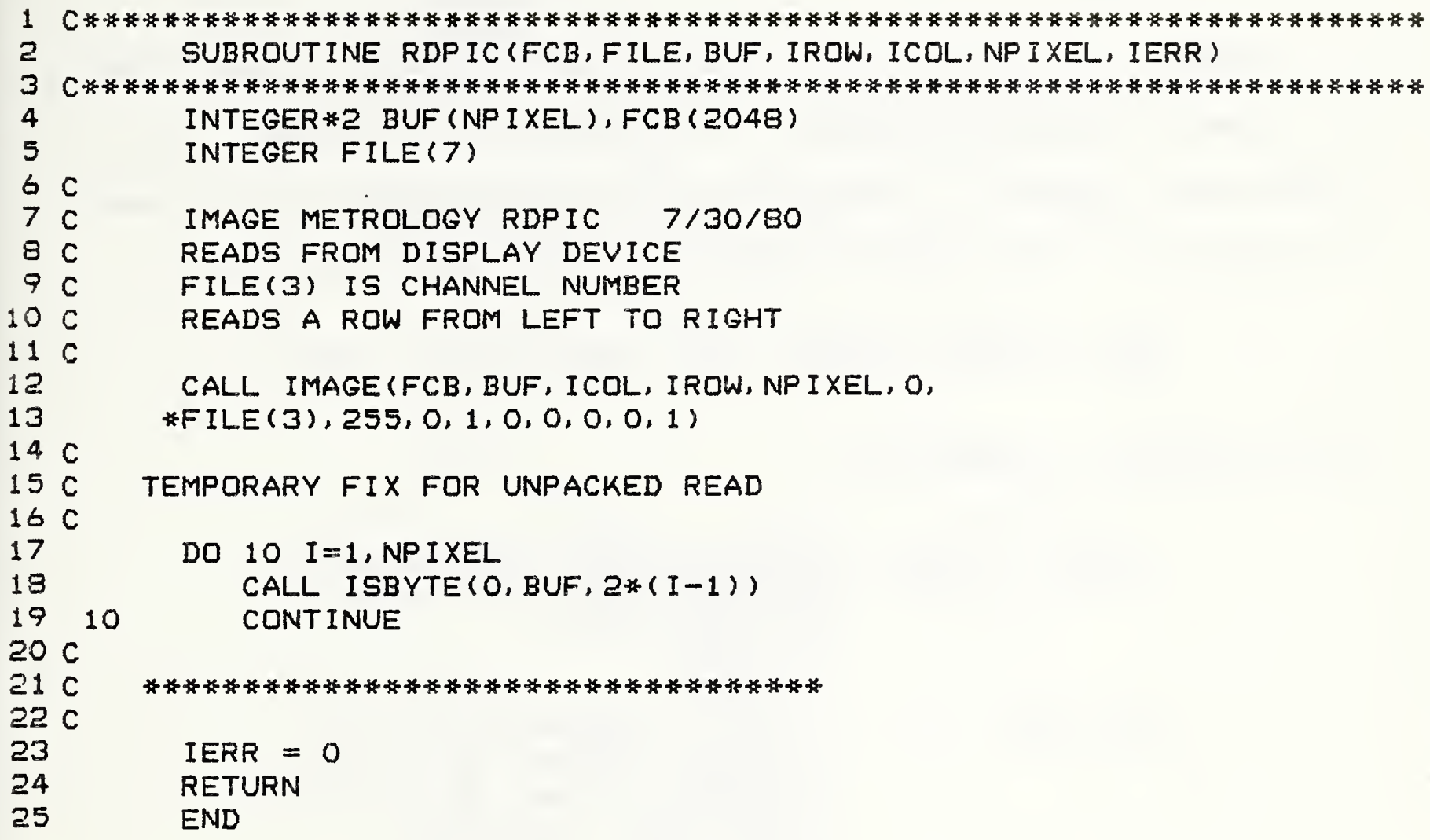


5.10.1 Summary

Based upon the direction vector $\vec{w}$ of the rays (either 1 ight or viewer) and the vertices of the rectangle of interest, this subroutine returns, in the arrays EX, EY, the extreme points seen by the rays. The cailing sequence for this subroutine is:

CALL EXTREM (IWCASE, YRX, VFY, EX, EY, IFLE) .

The parameters passed are:

\begin{tabular}{|c|c|c|}
\hline IWCASE & - & $\begin{array}{l}\text { A case number that depends on } \\
\text { the signs and magnitudes of } \\
\text { W(1) and W(2). } \\
\text { INTEGER }\end{array}$ \\
\hline VFX, & - & $\begin{array}{l}X \text { and } Y \text { components of the } \\
\text { vertices of the rectangle of } \\
\text { interest. Starting in the } \\
\text { upper left corner and } \\
\text { proceeding counterclockwise } \\
\text { the vertices are indexed: } \\
(1,1),(2,1),(2,2),(1,2) \text {. } \\
\text { REAL arrays }\end{array}$ \\
\hline$E X, E Y$ & - & $\begin{array}{l}x \text { and } y \text { components of the } \\
\text { extreme values. There are } \\
\text { only two in each case. } \\
\text { REAL arrays }\end{array}$ \\
\hline IFLG & - & $\begin{array}{l}\text { Error fiag: Set to } 1 \text { if } \\
W(1)=\omega(2)=0,0 \text { otherwise. } \\
\text { INTEGER }\end{array}$ \\
\hline
\end{tabular}

ERTREH does not call ary subroutines. 
5.10.2 Flow Chart

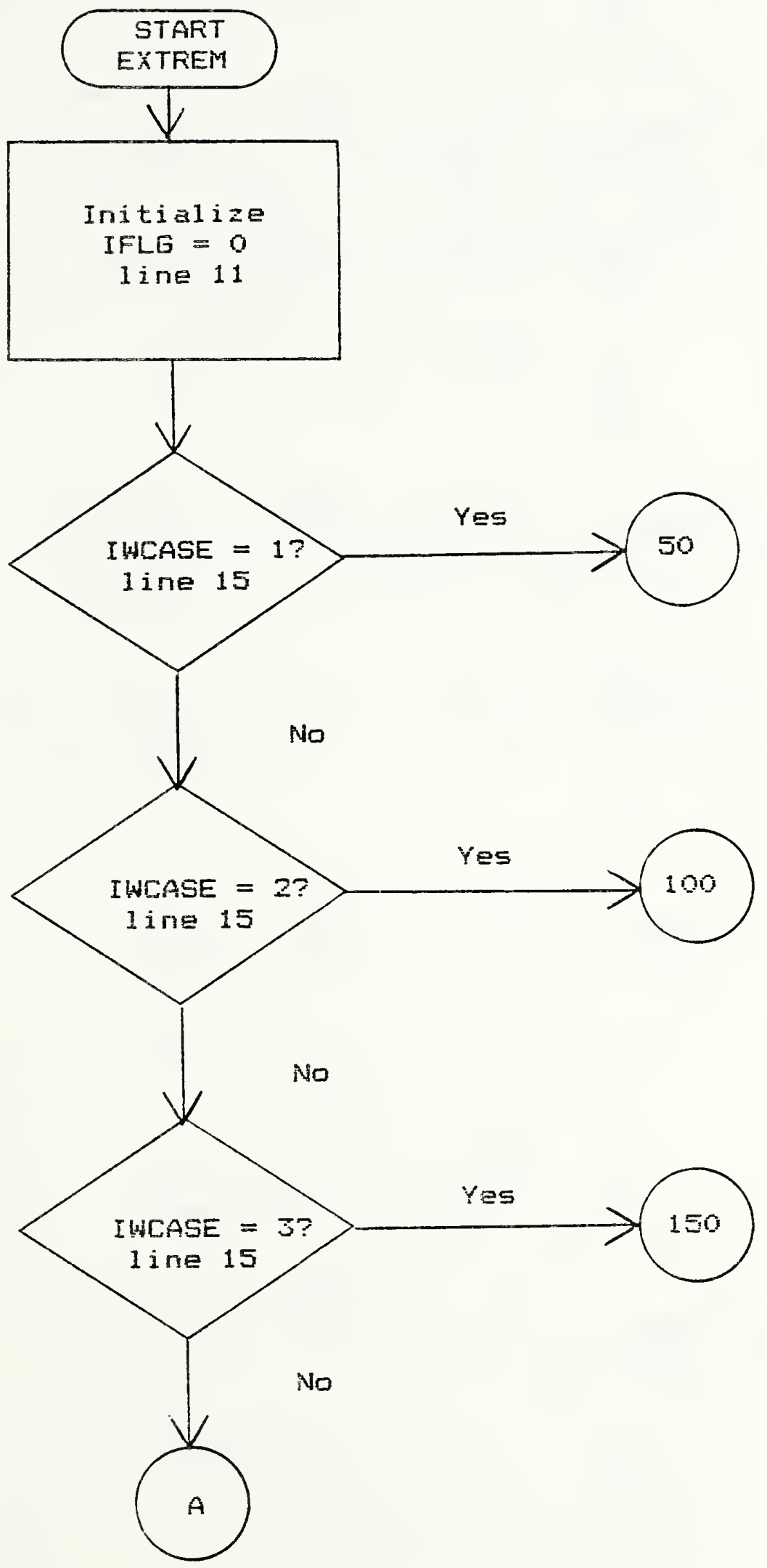




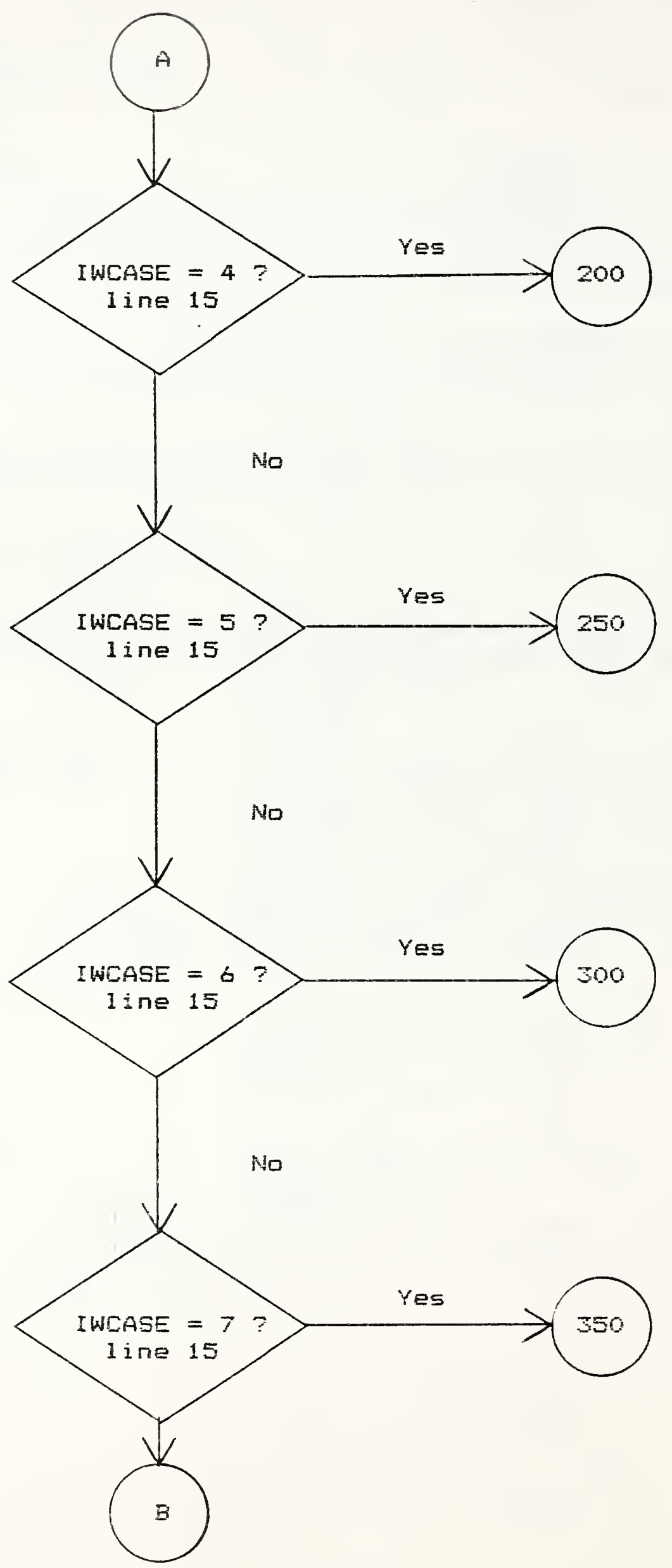




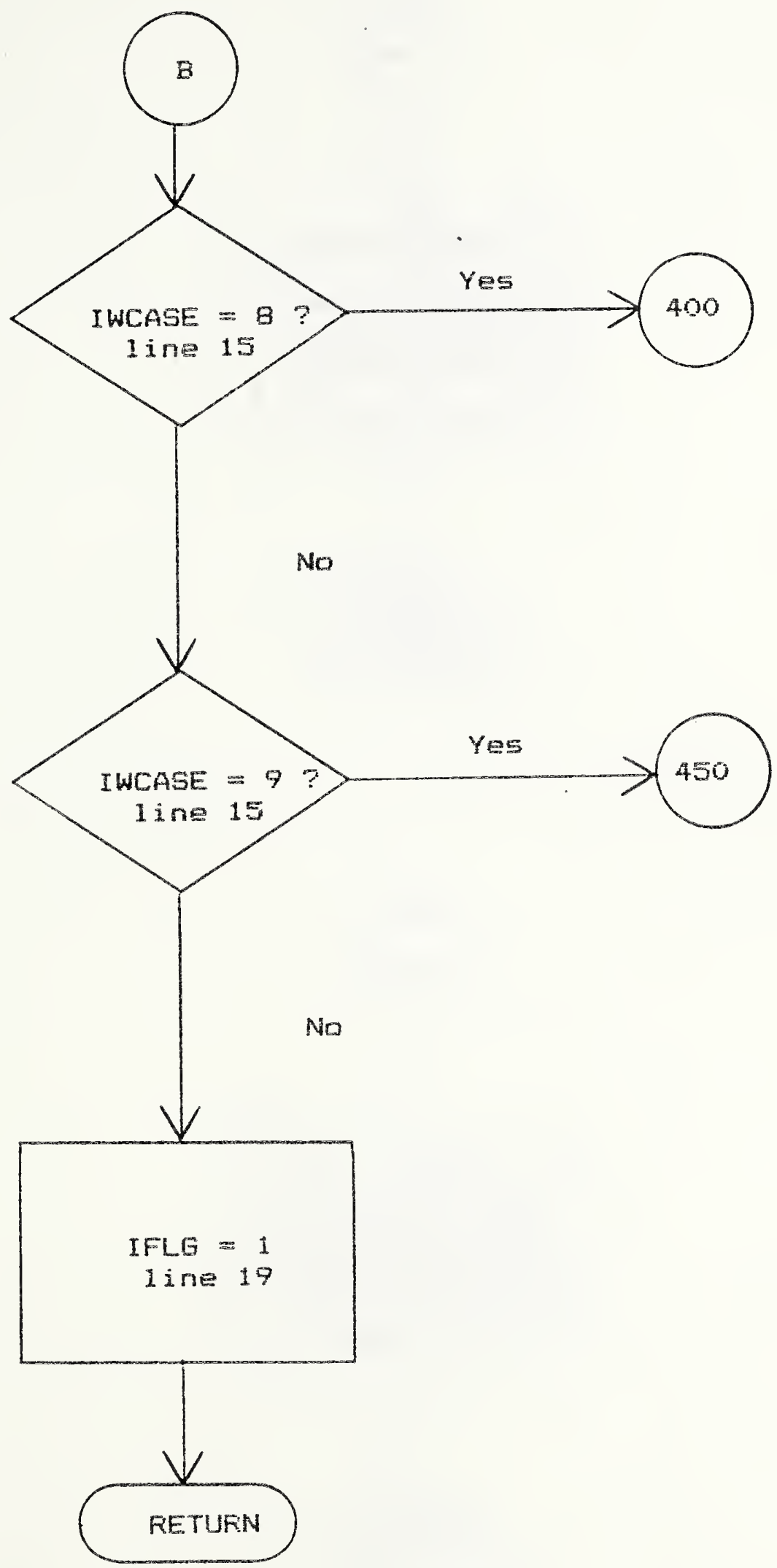



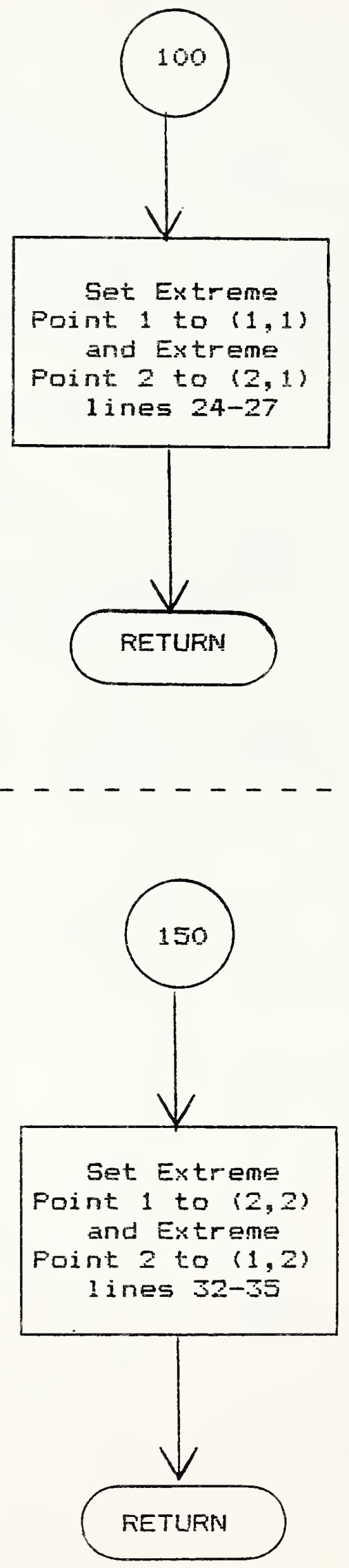

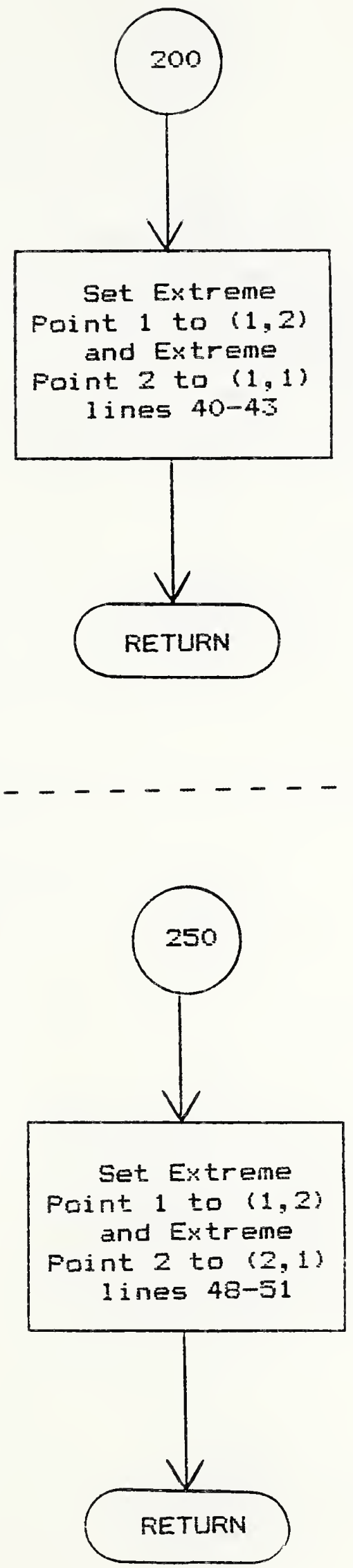

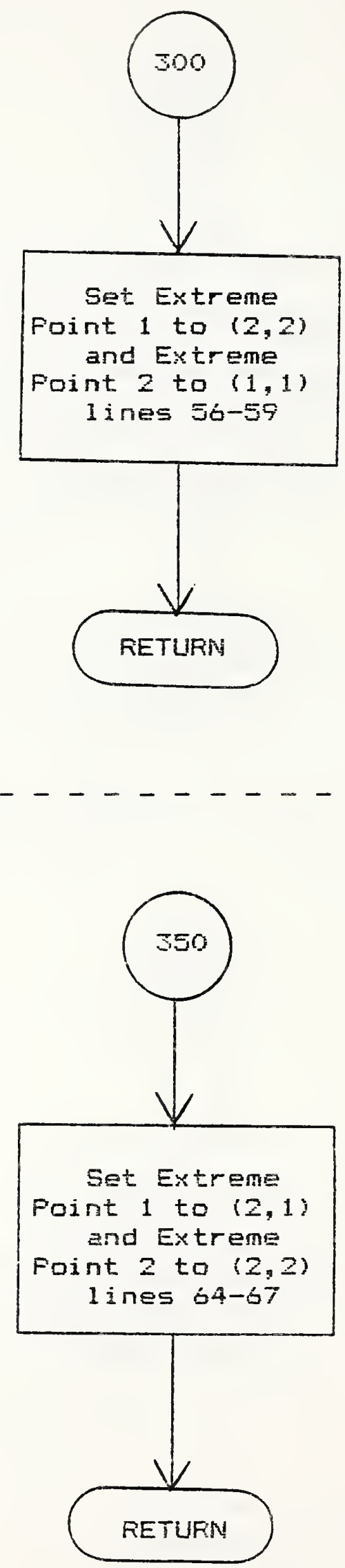

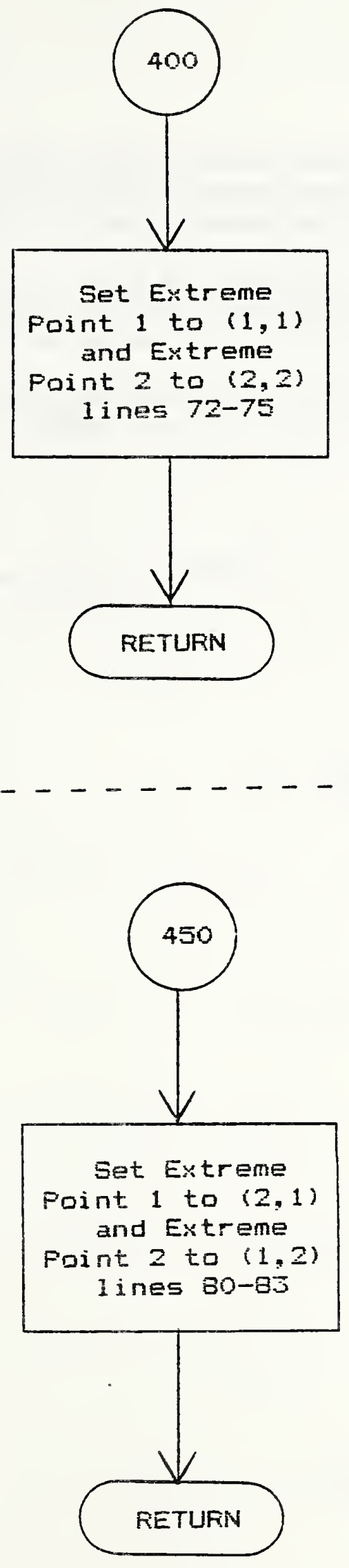
1 C 2 SUBROUTINE EXTREM (IWCASE, VRX, VRY, EX, EY, IFLG)

3 C

$4 \mathrm{C}$

5 C BASED UPON THE DIRECTION VECTOR $W$ OF THE RAYS OF INTEREST AND

6 C THE VERTICES DF THE PICTURE RECTANGLE THIS SUBROUTINE RETURNS

7 C THE EXTREME POINTS SEEN BY THE RAYS IN THE ARRAYS EX, EY

$8 \mathrm{C}$

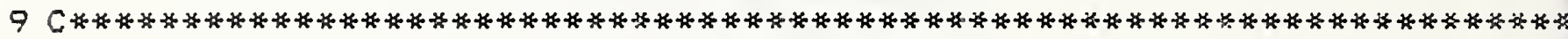

10

11

REAL VRX(2), VRY(2), EX(2), EY(2)

$12 \mathrm{C}$

13 C BRANCH DN IWCASE

$14 \mathrm{C}$

15 GO TO $(50,100,150,200,250,300,350,400,450)$, IWCASE

$16 \mathrm{C}$

17 C CASE 1: $W(1)=W(2)=0$

$18 \mathrm{C}$

1950 IFLG $=1$

20 RETURN

$21 \mathrm{C}$

22 C CASE $2: w(1)=0, w(2)>0$

$23 \mathrm{C}$

$24100 \quad E X(1)=\operatorname{VRX}(1)$

$25 \quad E Y(1)=\operatorname{VRY}(1)$

$26 \quad E X(2)=V R X(2)$

$27 \quad E Y(2)=$ VRY (1)

28 RETLIRN

$29 \mathrm{C}$

$30 C \operatorname{CASE} 3: w(1)=0, w(2)<0$

$31 \mathrm{C}$

$32150 \quad E X(1)=V R X(2)$

$33 \quad E Y(1)=\operatorname{VRY}(2)$

$34 \quad E X(2)=\operatorname{VRX}(1)$

$35 \quad E Y(2)=V R Y(2)$

36 RETURN

$37 \mathrm{C}$

38 CASE 4: $w(1)>0, w(2)=0$

$39 \mathrm{C}$

$40200 \quad E X(1)=\operatorname{VR} X(1)$

$41 \quad E Y(1)=\operatorname{VRY}(2)$

$42 \quad E X(2)=\operatorname{VRX}(1)$

$43 \quad E Y(2)=\operatorname{VRY}(1)$

44 RETURN

$45 \mathrm{C}$

46 C CASE 5: $w(1)>0, W(2)>0$

$47 \mathrm{C}$

$48250 \quad E X(1)=\operatorname{VRX}(1)$

$49 \quad E Y(1)=\operatorname{VRY}(2)$

$50 \quad E X(2)=\operatorname{VRX}(2)$ 


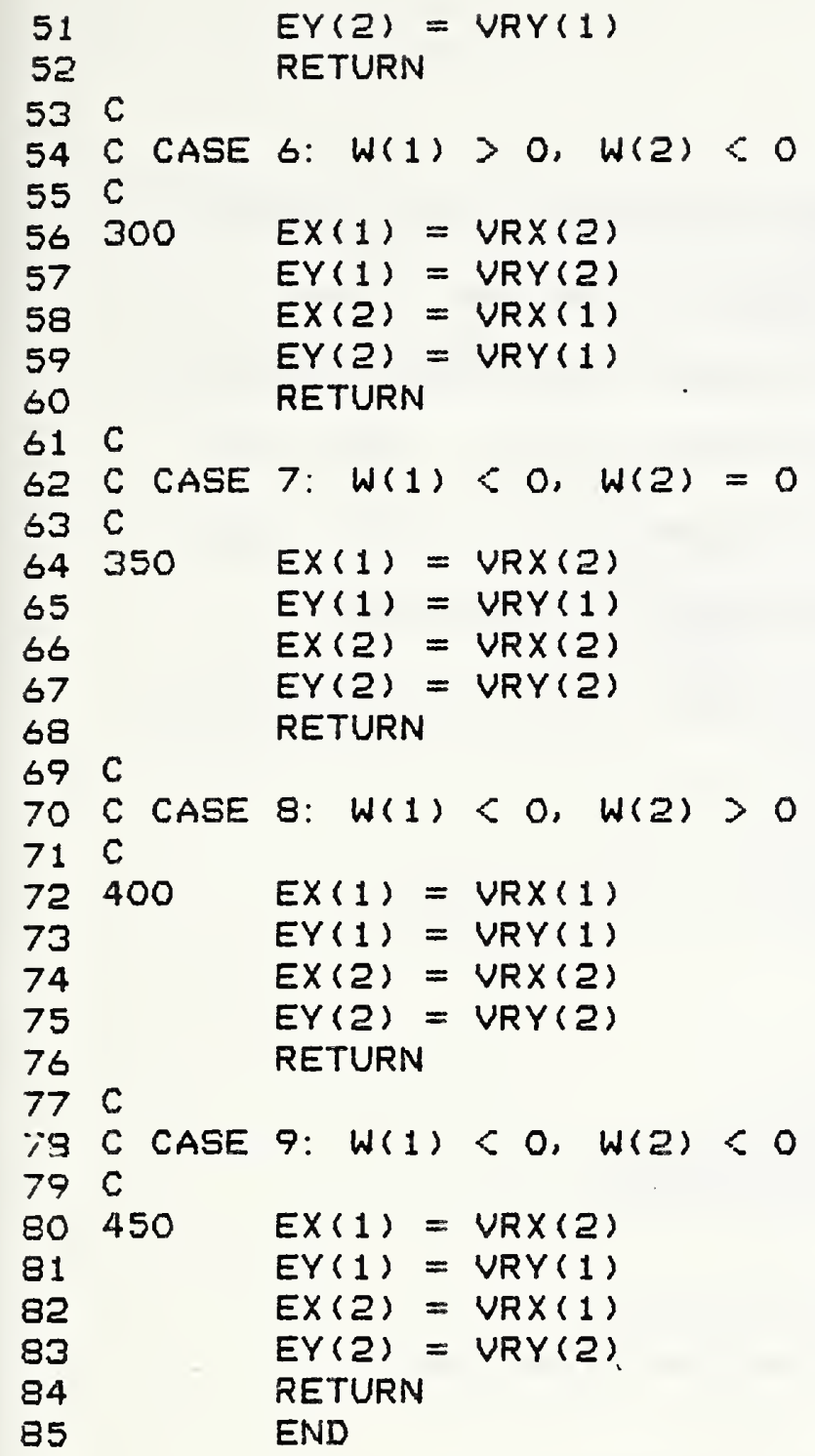




\subsection{1 .1 Summary}

Let the world coordinate point $(X O, Y O, Z O)$ be projected to the screen point $(S Y O, S \times O)$ by a parallel projection. Note that the screen coordinate system is an inverted coordinate system so that syo represents the row of the projected point. Then the unit vector $\vec{k}$ sitting at $(x O, Y O, z O)$ is directed in such a way that its coefficients represent an increment or decrement of a row number from the initial row set by syo. The calling sequence is:

CALL EETRCW (XO, YO, ZO, SYO, $K, X, Y, Z, S Y$.

The parameters passed are:

\begin{tabular}{|c|c|c|}
\hline$X 0, Y 0,20$ & - & $\begin{array}{l}\text { Components of the center of } \\
\text { solid of interest. } \\
\text { REAL }\end{array}$ \\
\hline SYo & - & $\begin{array}{l}\text { Frojection row of } \\
\text { XO, YO, ZO on the viewplane. } \\
\text { REAL }\end{array}$ \\
\hline$k$ & - & $\begin{array}{l}\text { Unit vector directed in } \\
\text { such a way that coefficients } \\
\text { index screen rows. } \\
\text { REAL Array }\end{array}$ \\
\hline$X, Y, Z$ & - & $\begin{array}{l}\text { Foint for which row must be } \\
\text { found. } \\
\text { REAL }\end{array}$ \\
\hline $5 Y$ & - & $\begin{array}{l}\text { Screen row for } X, Y, Z \text {. } \\
\text { FEAL }\end{array}$ \\
\hline
\end{tabular}

GETFOW ca11s no subroutines. 
5.11.2 Flow Chart

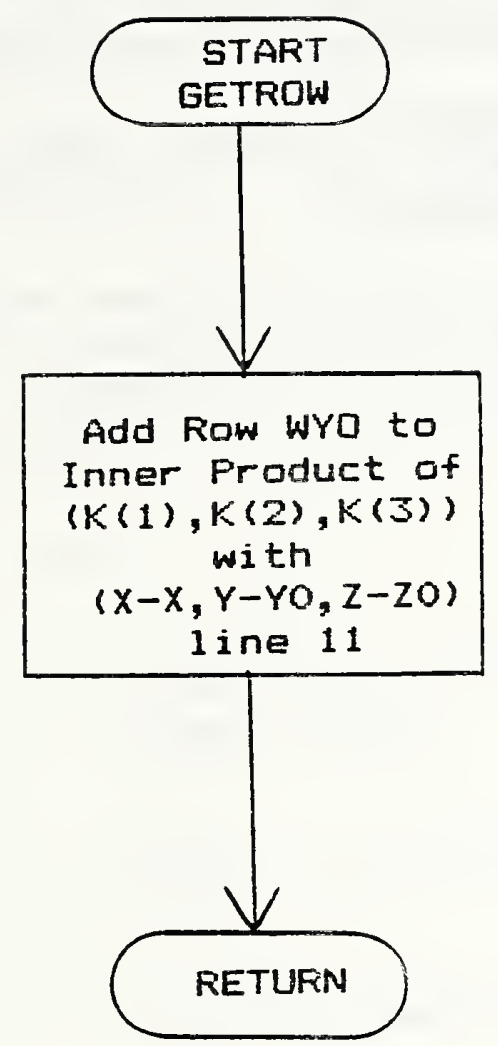




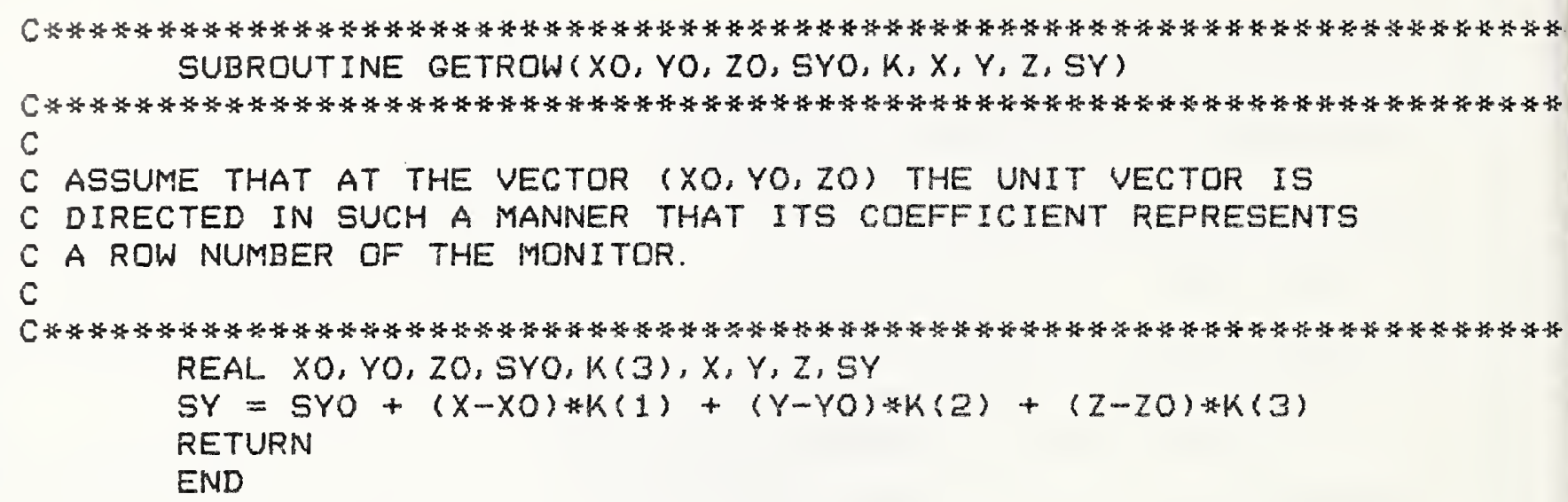




\subsection{Summary}

Given a point $(X, Y)$ on the world coordinate $Z=0$ plane, this subroutine returns the $Z$ value at $(X, Z)$ on a directed line row indexed by $V$. The calling sequence for this subroutine is:

$$
\text { CALL GETZ }(X, Y, V, K, Z) \text {. }
$$

The parameters passed are:

$$
\begin{array}{ll}
X, Y \quad-\quad & \begin{array}{l}
\text { Components of the } Z=0 \\
\text { plane point. } \\
\text { REAL }
\end{array} \\
V \quad \text { Row index specified. } \\
\\
\text { FEAL }
\end{array}
$$

GETZ does not call any subroutines. 
5.12.2 Flow Chart

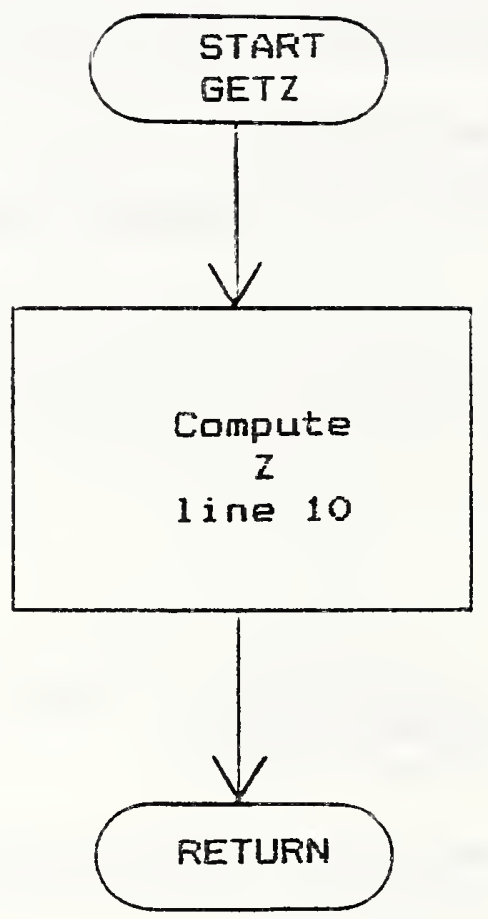


5. 12.3 Listing

1

2

3

$4 \mathrm{C}$

5 C GIVEN $\cdot(X, Y)$ THIS SUBRQUTINE RETURNS THE $Z$-VALUE AT ( $X, Y$ ) ALQNG

6 C THE RAY INDEXED BY $V$

$7 \mathrm{C}$

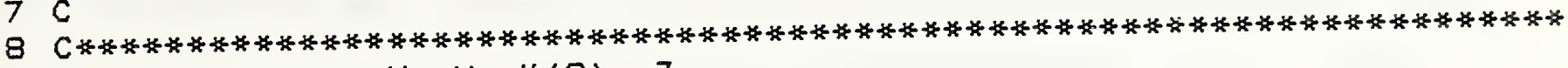

$9 \quad$ REAL $X, Y, V, K(3), Z$

$Z=(1 . / K(3)) *(V-X * K(1)-Y * K(2))$

RETURN

END 


\subsection{Subroutine GETF}

\section{13.1 Summary}

Given $(X, Y, Z)$ in the world coordinate system, this subroutine returns the multiple of the unit vector $\vec{w}$ pointing along the ray that intercepts the $(X, Y, Z)$ point. The calling sequence for this subroutine is:

$$
\text { CALL GETR }(X, Y, Z, W, R) \text {. }
$$

The parameters passed are:

$$
\begin{array}{ll}
X, Y, Z \quad-\quad \begin{array}{l}
\text { World coordinate point. } \\
\text { REAL }
\end{array} \\
\text { w } \quad \text { Unit vector pointing } \\
\text { along rays. } \\
\text { REAL Array } \\
\text { R } \\
\quad \begin{array}{l}
\text { Multiple of w-vector. } \\
\text { REAL }
\end{array}
\end{array}
$$

GETR does not call any subroutines. 
5.13.2 Flow Chart

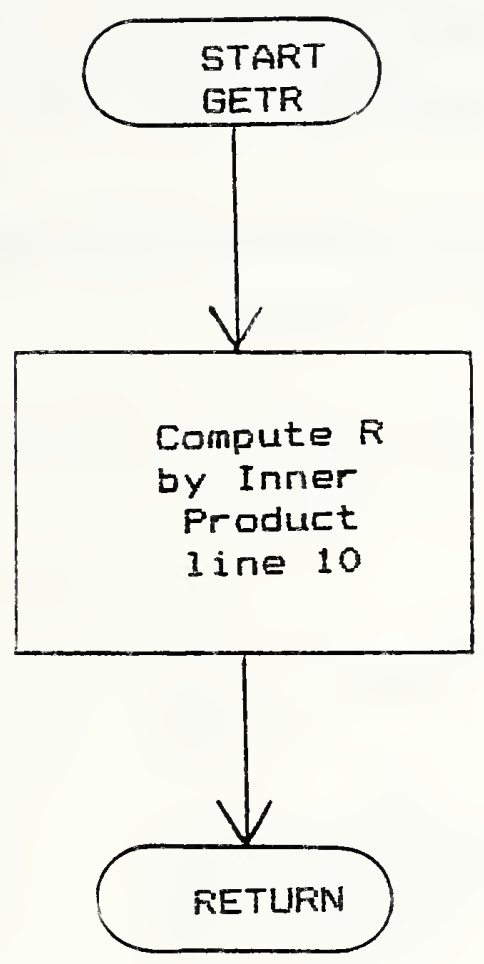




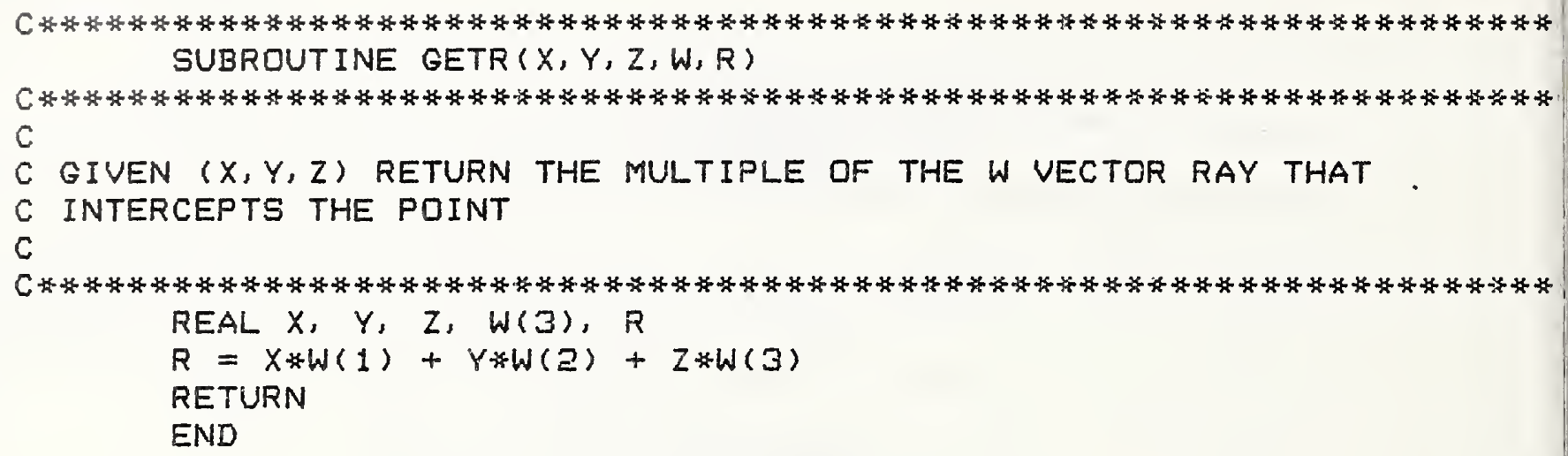


5. 14 Subroutine GETV

5.14.1 Summary

Given a point $(X, Y, Z)$ in the world coordinate system, this subroutine returns the multiple of the K-vector that indexes the ray that intercepts $(X, Y, Z)$. The calling sequence for this subroutine is:

CALL GETV $(X, Y, Z, K, V)$.

The parameters passed are:

$\begin{array}{ll}X, Y, Z \quad \text { - } & \text { World coordinate point. } \\ & \text { REAL } \\ K & \text { Vector used to index rays } \\ & \text { in a vertical column of } \\ & \text { the viewplane. } \\ & \text { REAL Array } \\ & \text { Multiple of } K \text { that indexes } \\ & \text { the vector. } \\ & \text { REAL. }\end{array}$

GETV does not call any subroutines. 
5.14.2 Flow Chart

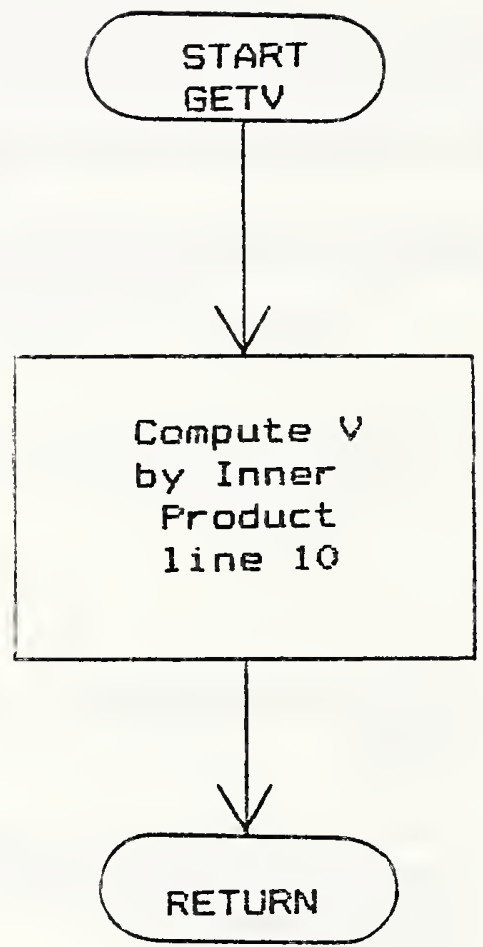


5.14.3 Listing

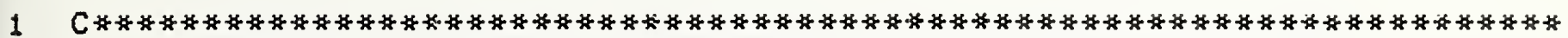
2 SUBRDUTINE GETV $(X, Y, Z, K, Y)$

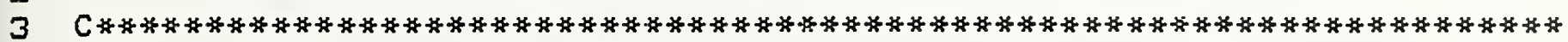
$4 C$

5 C GIVEN ( $X, Y, Z$ ) RETURN THE MULTIPLE DF THE $K$ VECTOR OF THE RAY

6 C THAT INTERCEPTS THE POINT

$7 \mathrm{C}$

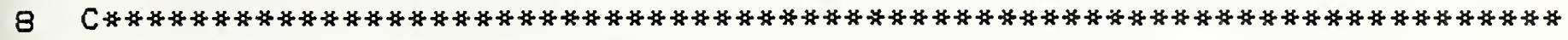
9 REAL $X, Y, Z, K(3), V$

$V=X * K(1)+Y * K(2)+Z * K(3)$

RETURN

END 
5.15.1 Summary

Given a point $(X, Y, Z)$ in the world coordinate system, this subroutine returns the multiple of the $\vec{K} \times \vec{W}$ vector for the ray that intercepts the point. In effect this selects the column or plane of rays that intersects $(X, Y, Z)$. The calling sequence for this subroutine is:

CALL GETH $(X, Y, Z, K X W, H)=$

The parameters passed are:

\begin{tabular}{|c|c|c|}
\hline$X, Y, Z$ & - & $\begin{array}{l}\text { Compoments of the world } \\
\text { coordinats system point. } \\
\text { REAL }\end{array}$ \\
\hline $\mathrm{K} \times w^{4}$ & - & $\begin{array}{l}\text { Vector orthogonal to the } \\
\text { K vector and lying in the } \\
\text { viewplane. } \\
\text { FEAL Array }\end{array}$ \\
\hline $\mathrm{H}$ & - & $\begin{array}{l}\text { Multiple of KoW } \\
\text { REAL }\end{array}$ \\
\hline
\end{tabular}

GETH does not cail any subroutines. 
5.15.2 Fl ow Chart

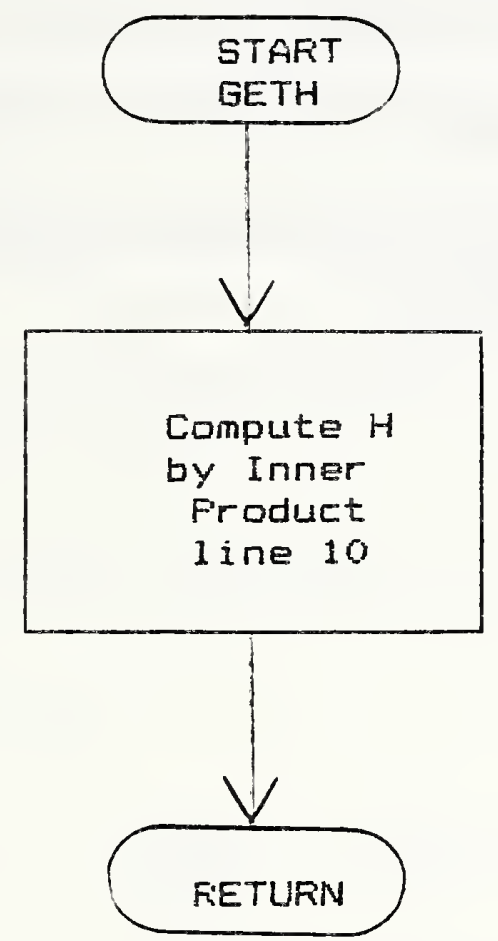




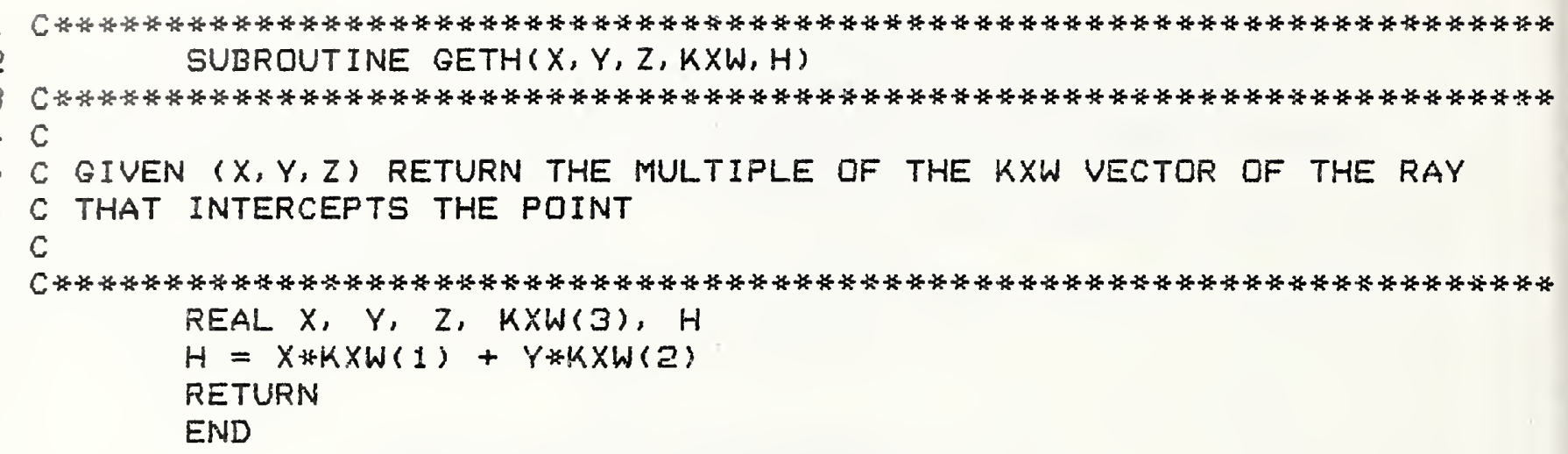

REAL $X, Y, Z, K X W(3), H$

$H=X * K X W(1)+Y * K X W(a)$

RETURN

END

12 
5.16 Subroutine GETXYZ

כ. í. 1 Summary

Let $(S Y O, S \times O)$ be the orthogonal projection sereen

coordinates of the point $(X O, Y O, Z O)$ in the world coordinate frame of reference. Let $(S Y, S X)$ be a given screen coordinate. This subroutine transforms the screen point (SY, SX) into its associated world coordinate system point $(X, Y, Z)$. The callirig sequence for this subrautine is:

CALL GETXYZ (XO, YO, ZO, SYO, SXO, SY, SX, K, KXW, $X, Y, Z)$.

The parameters passed ara:

\begin{tabular}{|c|c|c|}
\hline$X O, Y O, Z O$ & - & $\begin{array}{l}\text { Center of solid of interest. } \\
\text { REAL }\end{array}$ \\
\hline SYo, $5 \times 0$ & - & $\begin{array}{l}\text { Screen coordinates of the } \\
\text { projection of } x O, y 0,20 . \\
\text { REAL }\end{array}$ \\
\hline $5 y, 5 x$ & - & $\begin{array}{l}\text { Screen coordinates of the } \\
\text { selected screen paint. } \\
\text { REAL }\end{array}$ \\
\hline$k$ & - & $\begin{array}{l}\text { Vector Lised to select } \\
\text { screen row. } \\
\text { REAL Array }\end{array}$ \\
\hline$K \times H$ & - & $\begin{array}{l}\text { Vector used to select } \\
\text { screen column. } \\
\text { REAL Array }\end{array}$ \\
\hline$X, Y, Z$ & - & $\begin{array}{l}\text { World coordinate point } \\
\text { associated with SY, SX. } \\
\text { REAL }\end{array}$ \\
\hline
\end{tabular}

GETXYZ does not call any subroutines. 
5.16 .2 Flow Chart

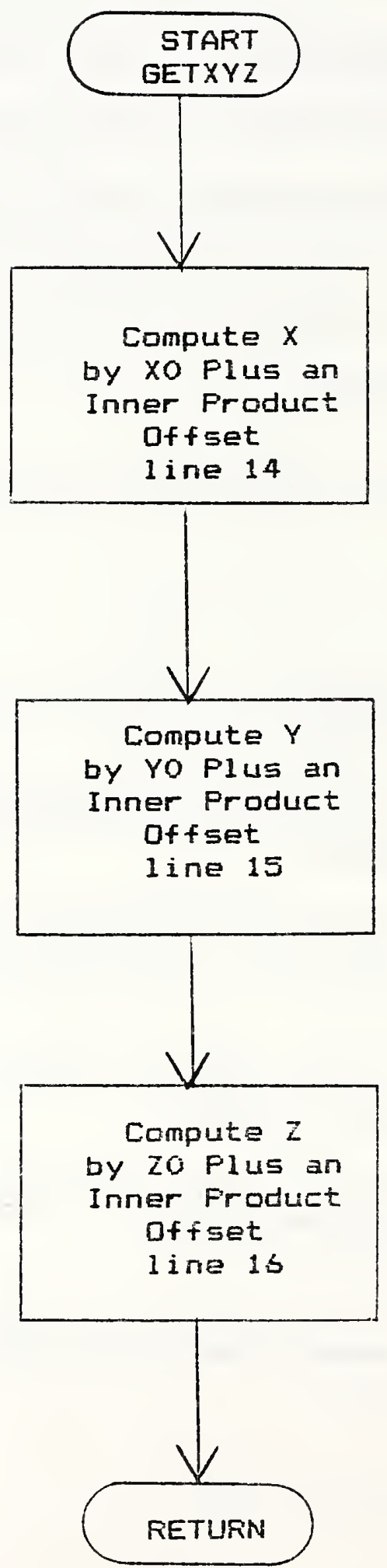




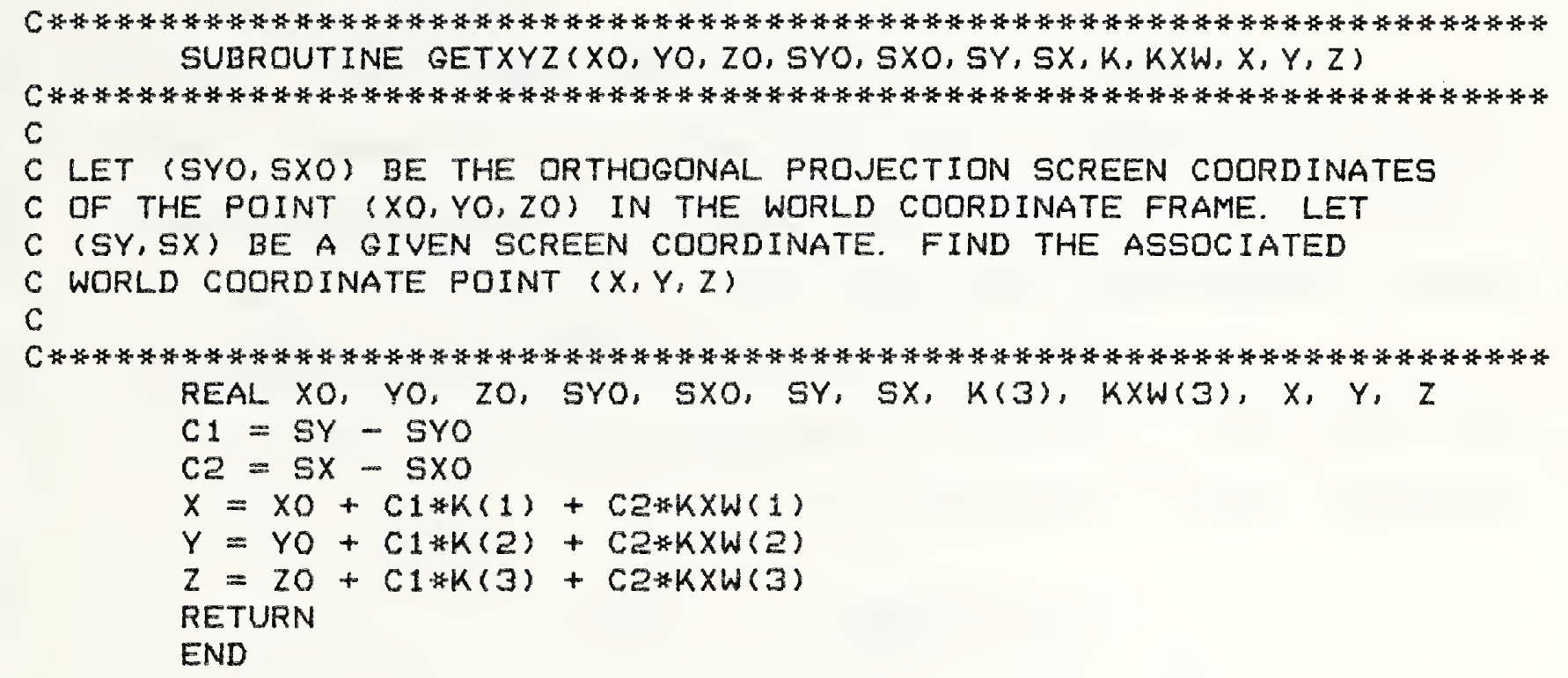




\subsubsection{Summary}

This subroutine returns an index number from 1 to 9 in the variable IWCASE. This index points to each possible case combination of the first two components of the $\overrightarrow{\hat{W}}$-vector that points along rays. The vector $(w(1), w(2))$ represents the direction vector of the projected directed Iine through the $Z=0$ plane in the world coordinate space. The calling sequence for this subroutine is:

$$
\text { CALL WCASE (W, IWCASE). }
$$

The parameters passed are:

\begin{tabular}{|c|c|c|c|}
\hline$W$ & - & $\begin{array}{l}\text { Direction vector } \\
\text { along rays. } \\
\text { REAL Array }\end{array}$ & pointing \\
\hline IWCASE & - & $\begin{array}{l}\text { Case number from } \\
\text { INTEGER }\end{array}$ & 1 to 7. \\
\hline
\end{tabular}

WEASE dOEs not call any subroutines. 
5.17.2 Flow Chart

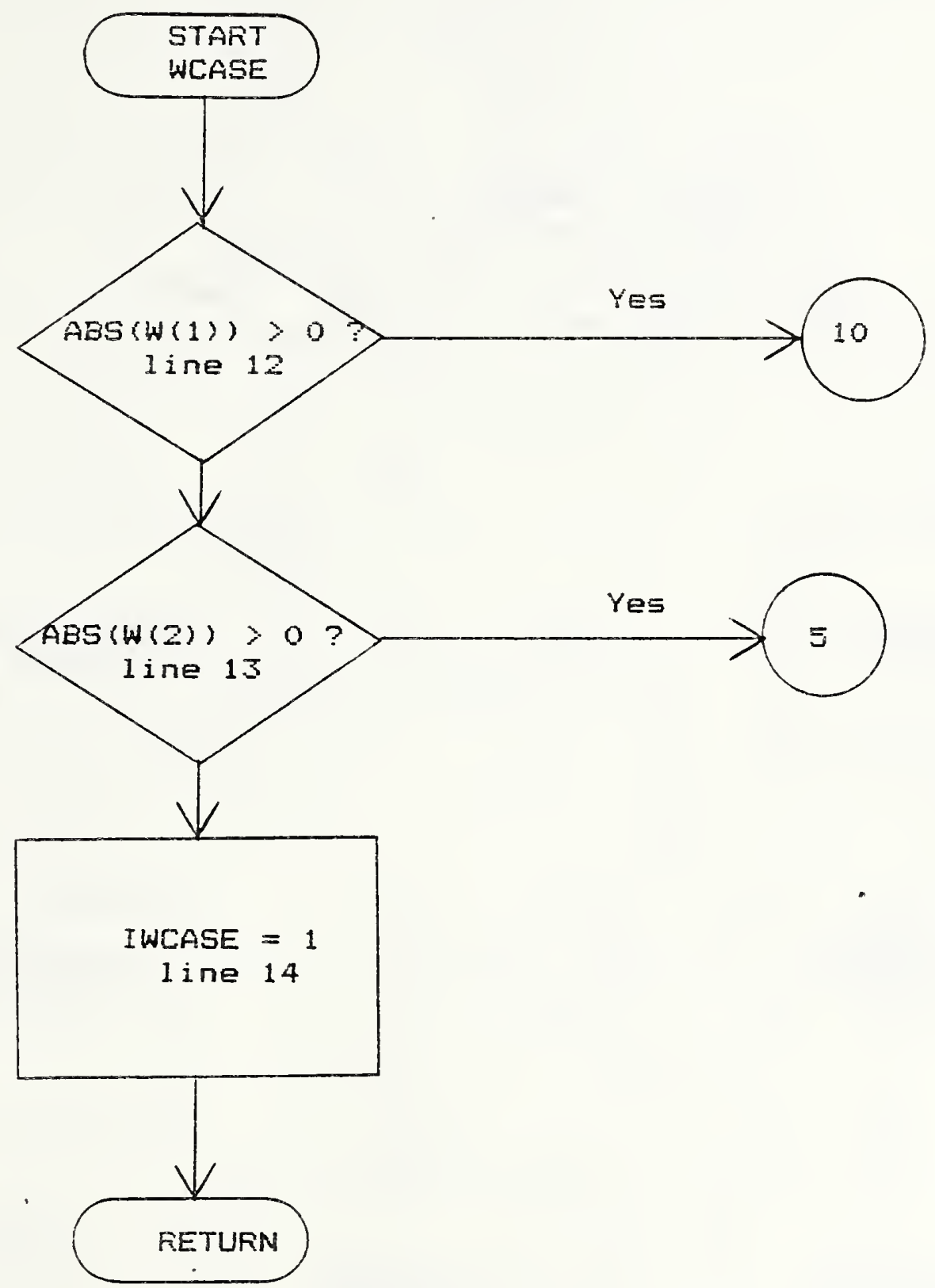




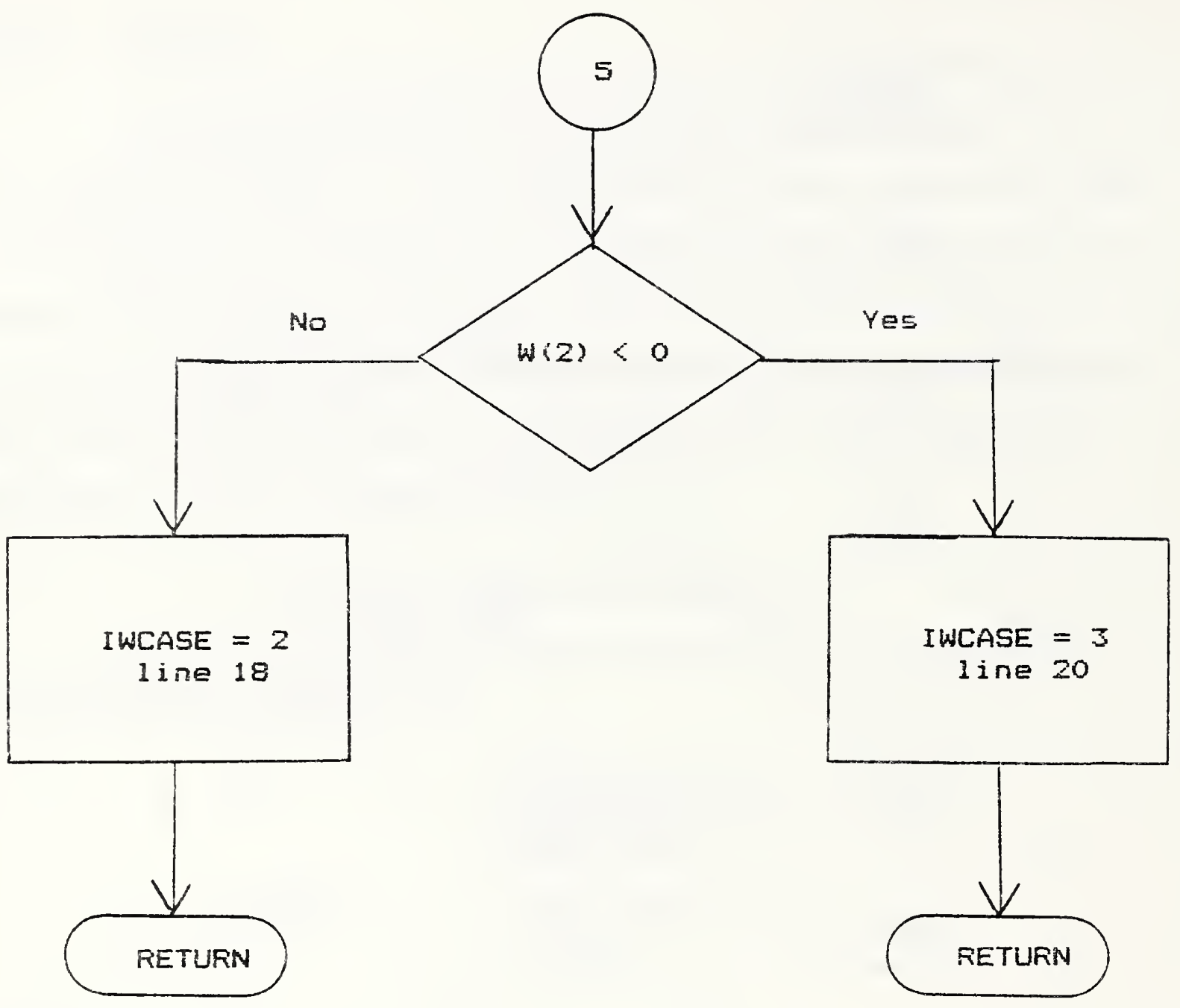




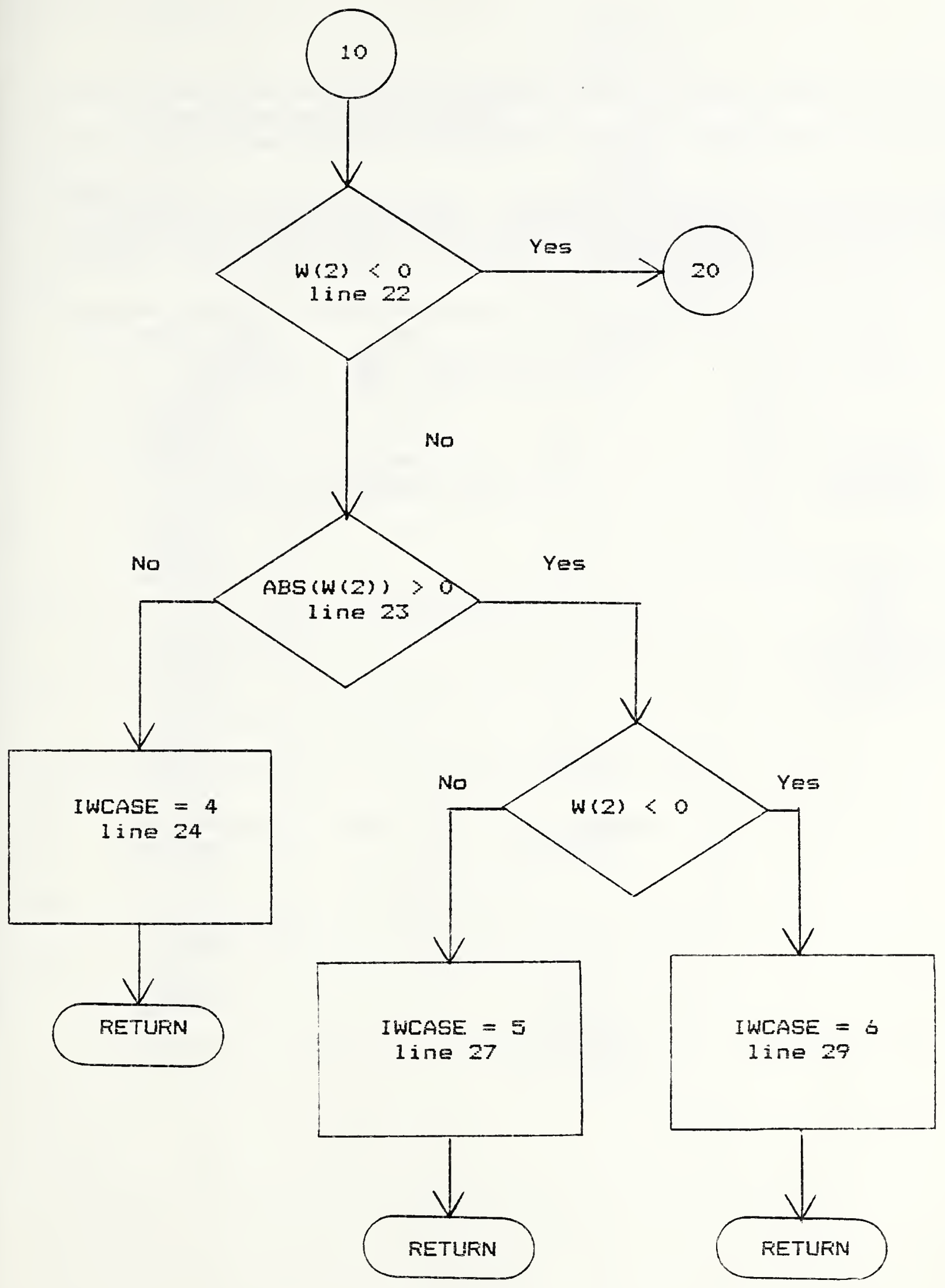




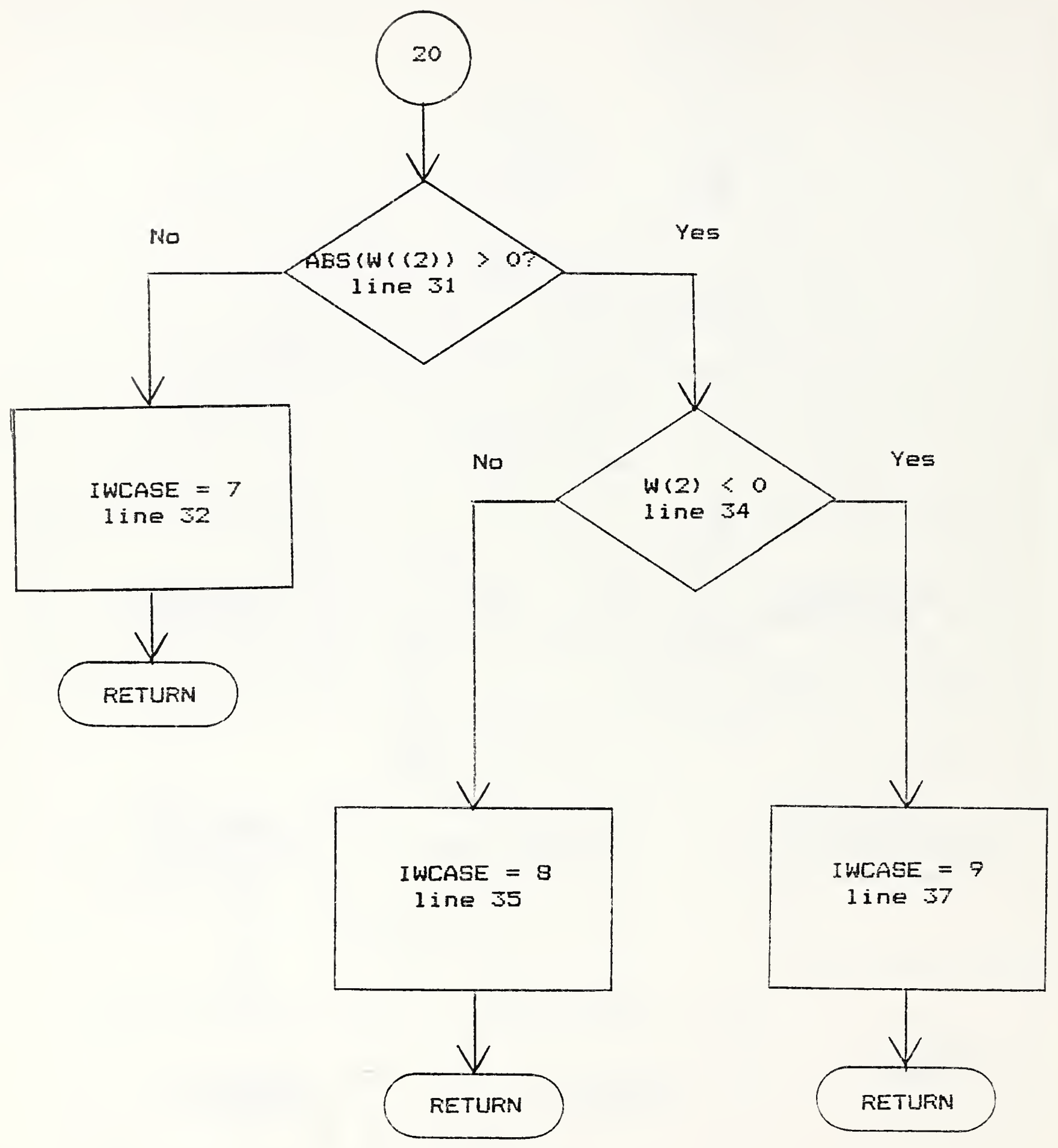


5.17.3 Listing

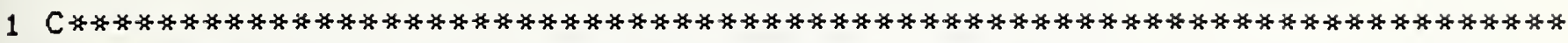
2

(

$4 \mathrm{C}$

5 C FUNCTION:

6 C TO RETURN. AN INDEX, IWCASE, THAT POINTS TO EACH POSSIBLE CASE

7 C COMBINATION DF THE FIRST TWO COMPONENTS OF THE W-VECTOR WHICH

8 C POINTS ALONG THE RAYS

$9 \mathrm{C}$

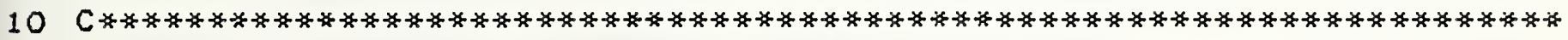

11

12

13

14

15

16

17

18

19

20

21

22

23

24

25

26

27

28

29

30

31

32

33

34

35

36

37

38

39
REAL W(3)

IF $(A B S(W(1))$. GE. 5. E-6) GQ TO 10

IF $(A B S(W(2))$. GE. 5. E-6) GO TO 5

IWCASE $=1$

RETURN

CONT INUE

IF (W(2) . LT. O. ) GO TO 7

IWCASE $=2$

RETURN

IWCASE $=3$

RETURN

10 IF (W(1).LT. O.) GO TO 20

IF $(A B S(W(2))$. GE. 5. E-6) GO TO 15

I WCASE $=4$

RETURN

15 IF (W(2).LT. O. ) GO TO 17

IWCASE $=5$

RETURN

IWCASE $=6$

RETURN

IF $(A B S(W(2))$. GE. 5.E-6) GO TO 25

IWCASE $=7$

RETURN

25 IF (W(2) . LT. O. ) GO TO 27

IWCASE $=8$

RETURN

27 IWCASE $=9$

RETURN

END 


\subsection{8 .1 Summary}

As the projections of the illuminating rays or viewing trace lines on the plane $Z=0$, some of the lines intersect the rectangle of interest. In the case of the shadowgraph, this rectangle is the base of the entire picture. In the case of the solid projection, it is the user selected rectangle. Assume that same projected ray enters tine rectangle at $(X, Y)$. This subroutine returns the next entry point or flags that an extreme point has been met. The calling sequence for this subroutine is:

CALL XYIN (IWCASE, EX, EY, IXIN, IYIN, XIN, YIN, IFLG).

The parameters passed through the calling sequence are:

ON INPUT -

\begin{tabular}{|c|c|c|}
\hline IWCASE & - & $\begin{array}{l}\text { The case index for } \vec{w} \text {. } \\
\text { INTEGER }\end{array}$ \\
\hline$E X, E Y$ & - & $\begin{array}{l}\text { Two element arrays representing } \\
\text { extreme points. } \\
\text { REAL Arrays }\end{array}$ \\
\hline IXIN,IYIN & - & $\begin{array}{l}\text { On entry to the subroutine these } \\
\text { represent the current entry } \\
\text { point to the rectangle: } \\
\text { INTEGER }\end{array}$ \\
\hline$X I N, Y I N$ & - & $\begin{array}{l}\text { Real values of IXIN, IYIN. } \\
\text { REAL }\end{array}$ \\
\hline
\end{tabular}

ON DUTPUT -

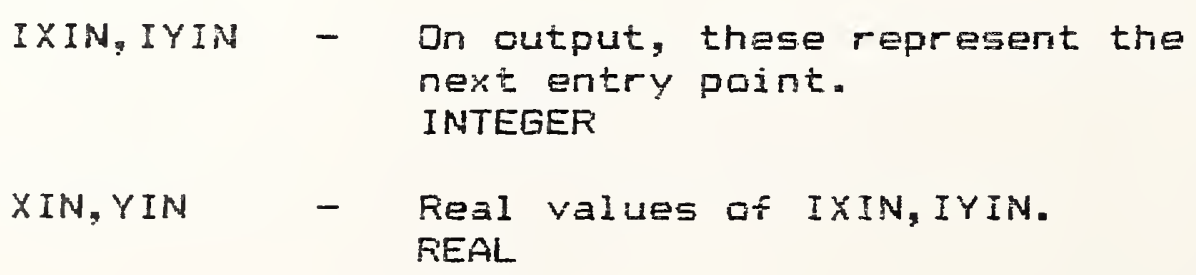


IFLG

- = 0 if a new entry point

is returned.

$=1$ if $W(1)=W(2)=0$.

$=2$ if the extreme point

$E X(2), E Y(2)$ is met.

XYIN does not call any subroutines. 


\subsection{8 .2 Flow Chart}

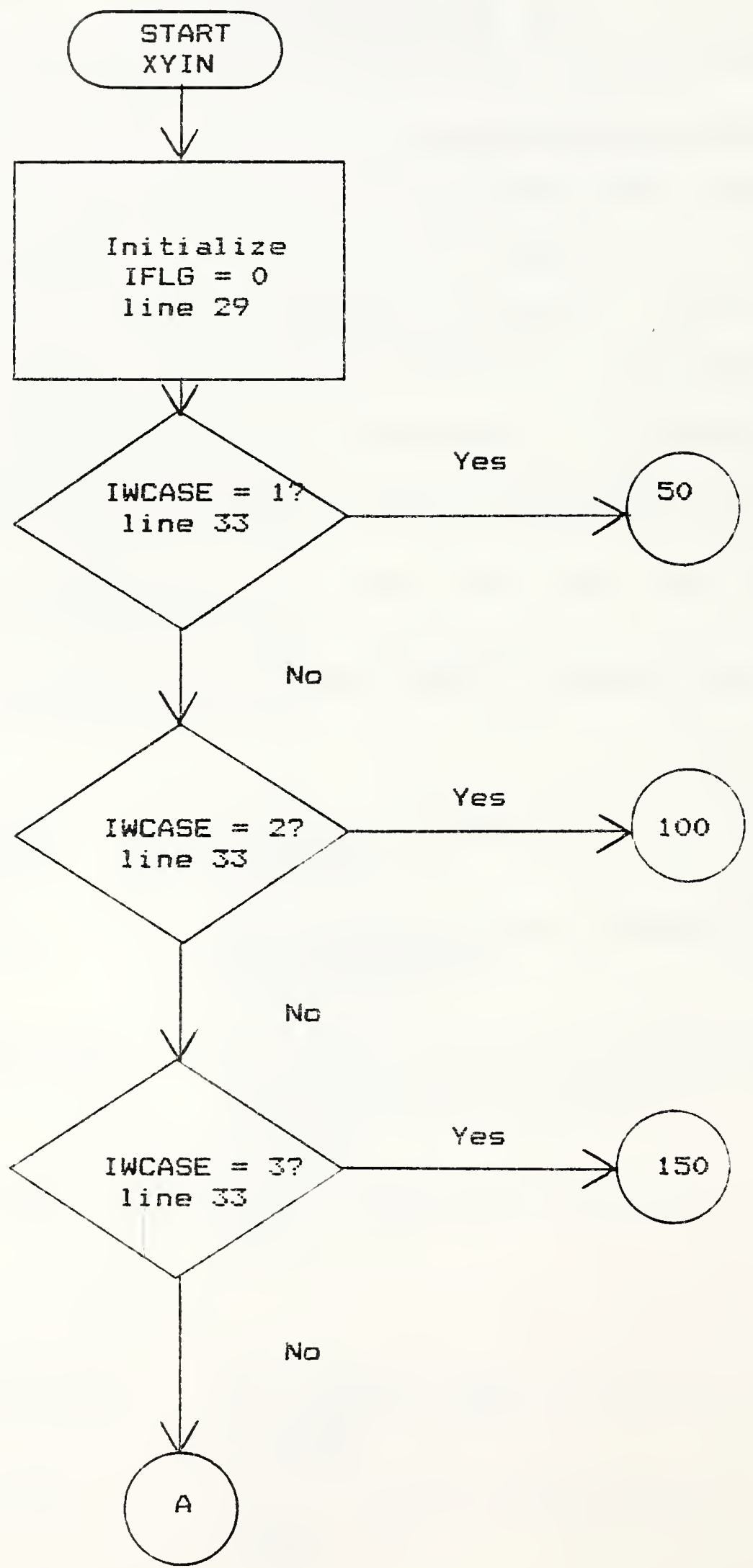




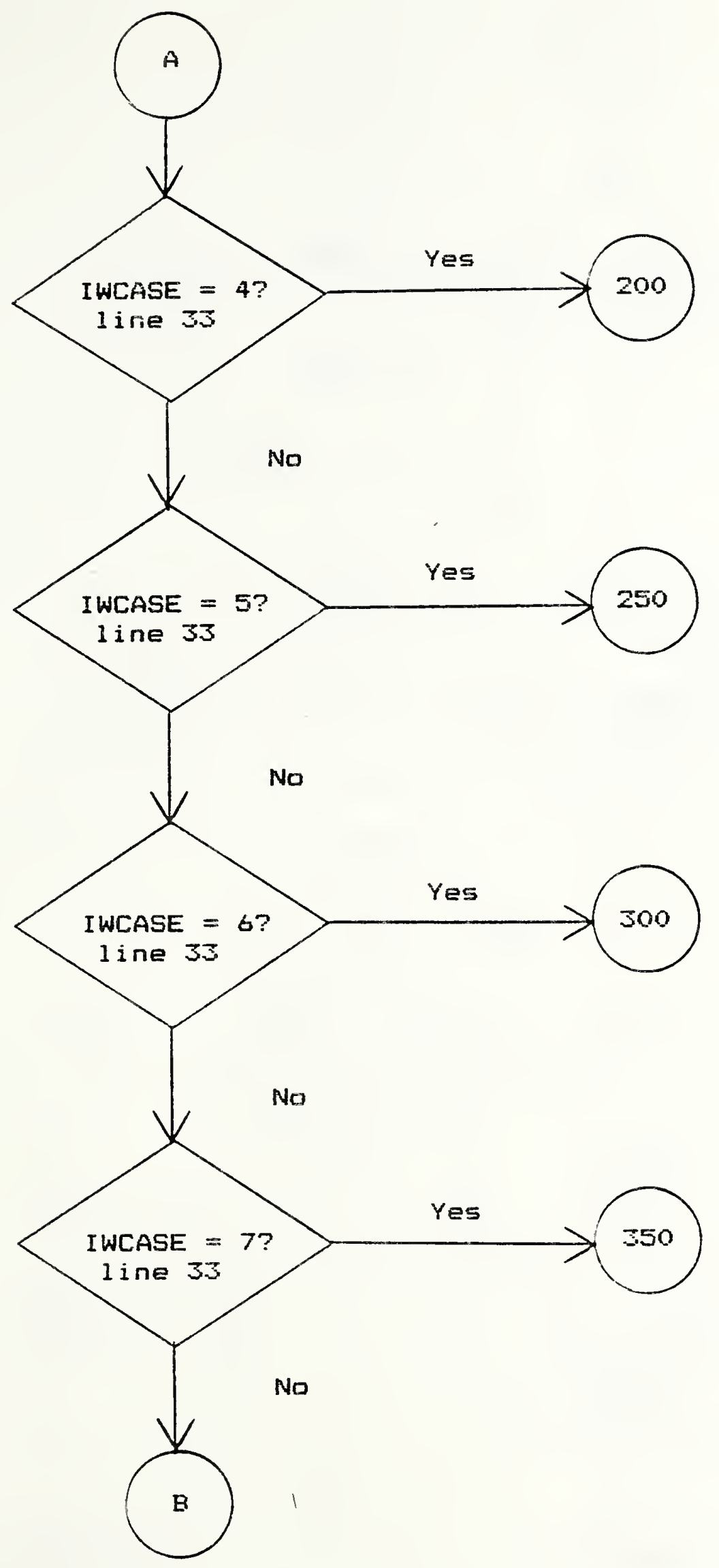




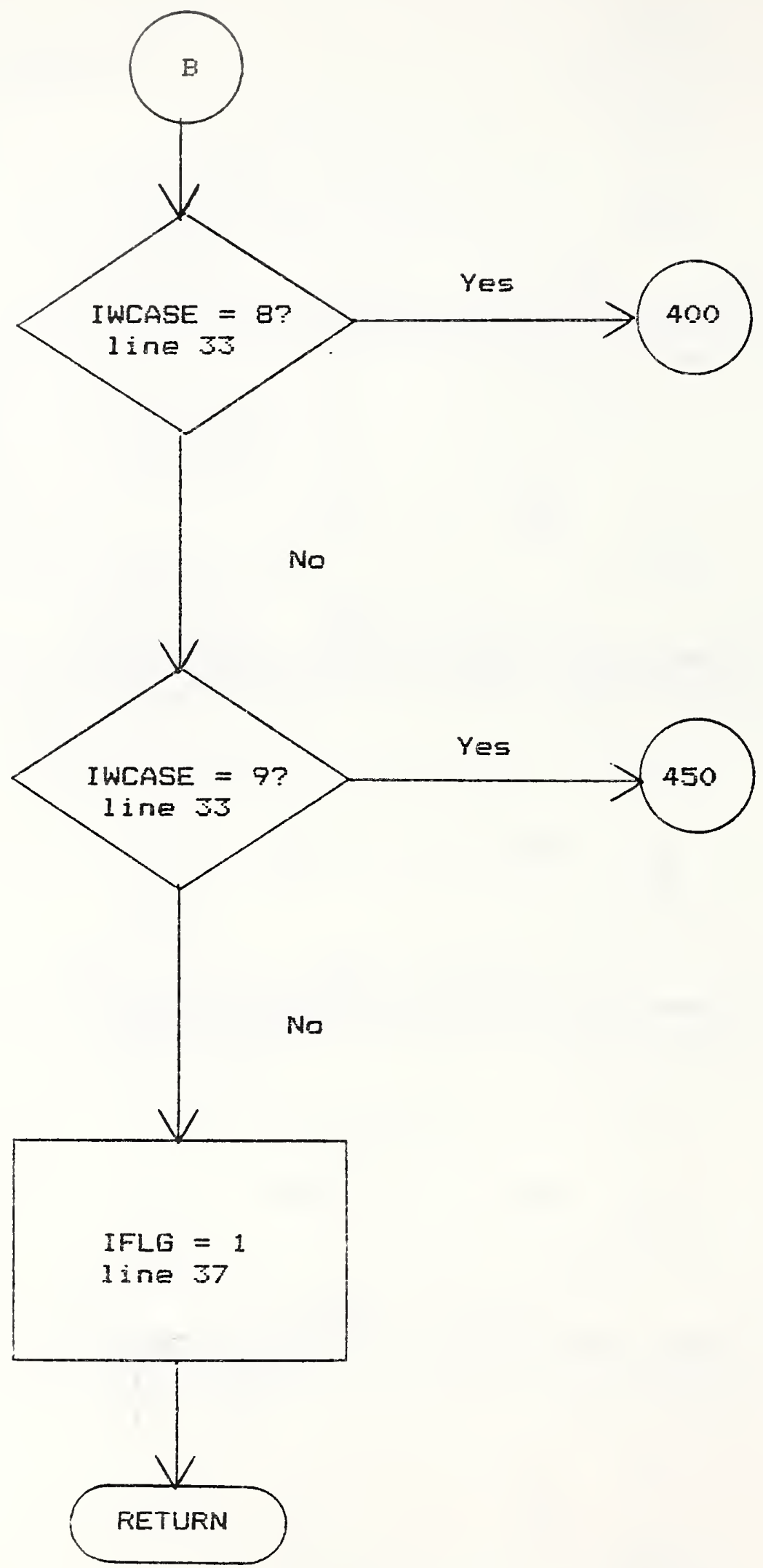



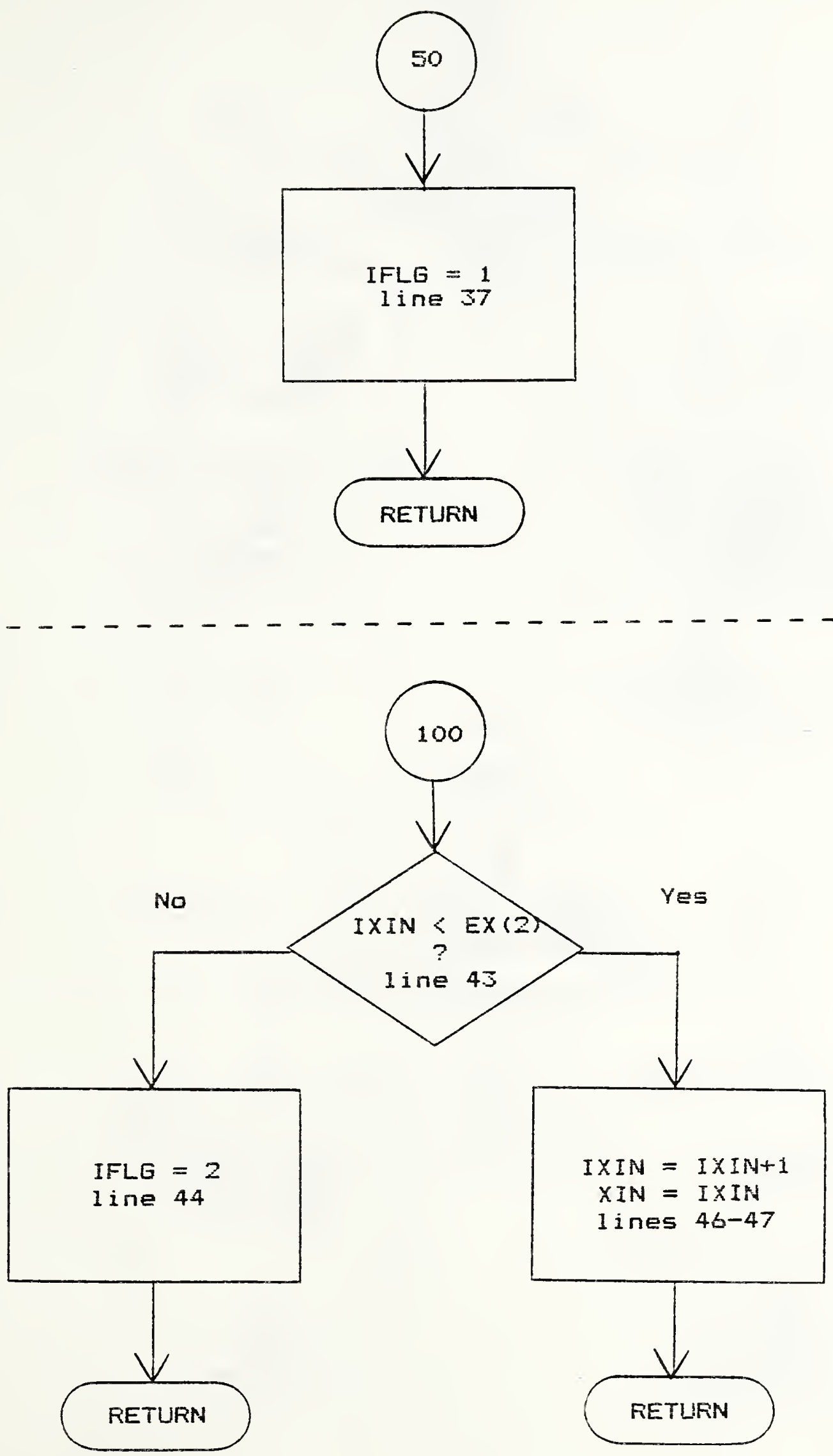

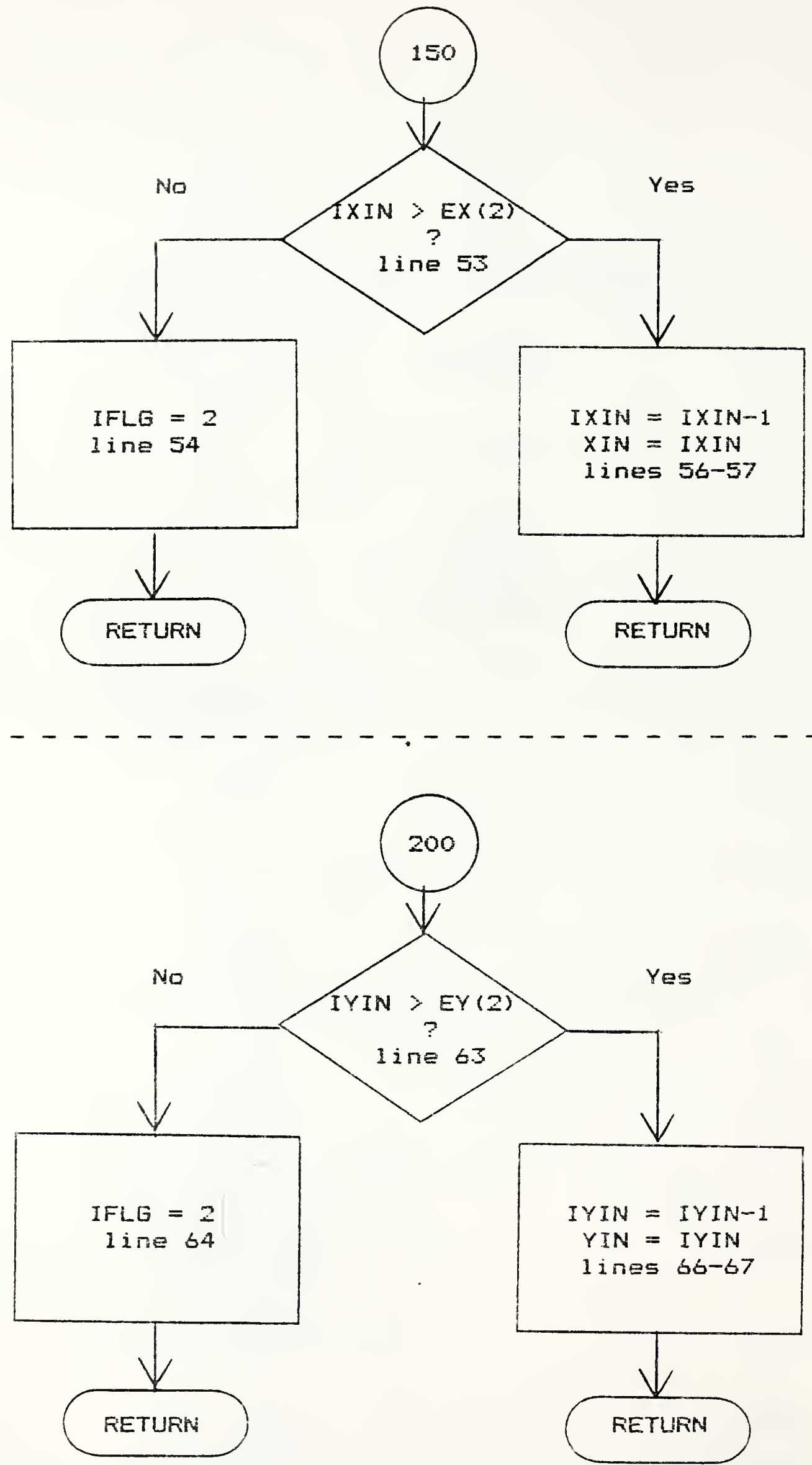


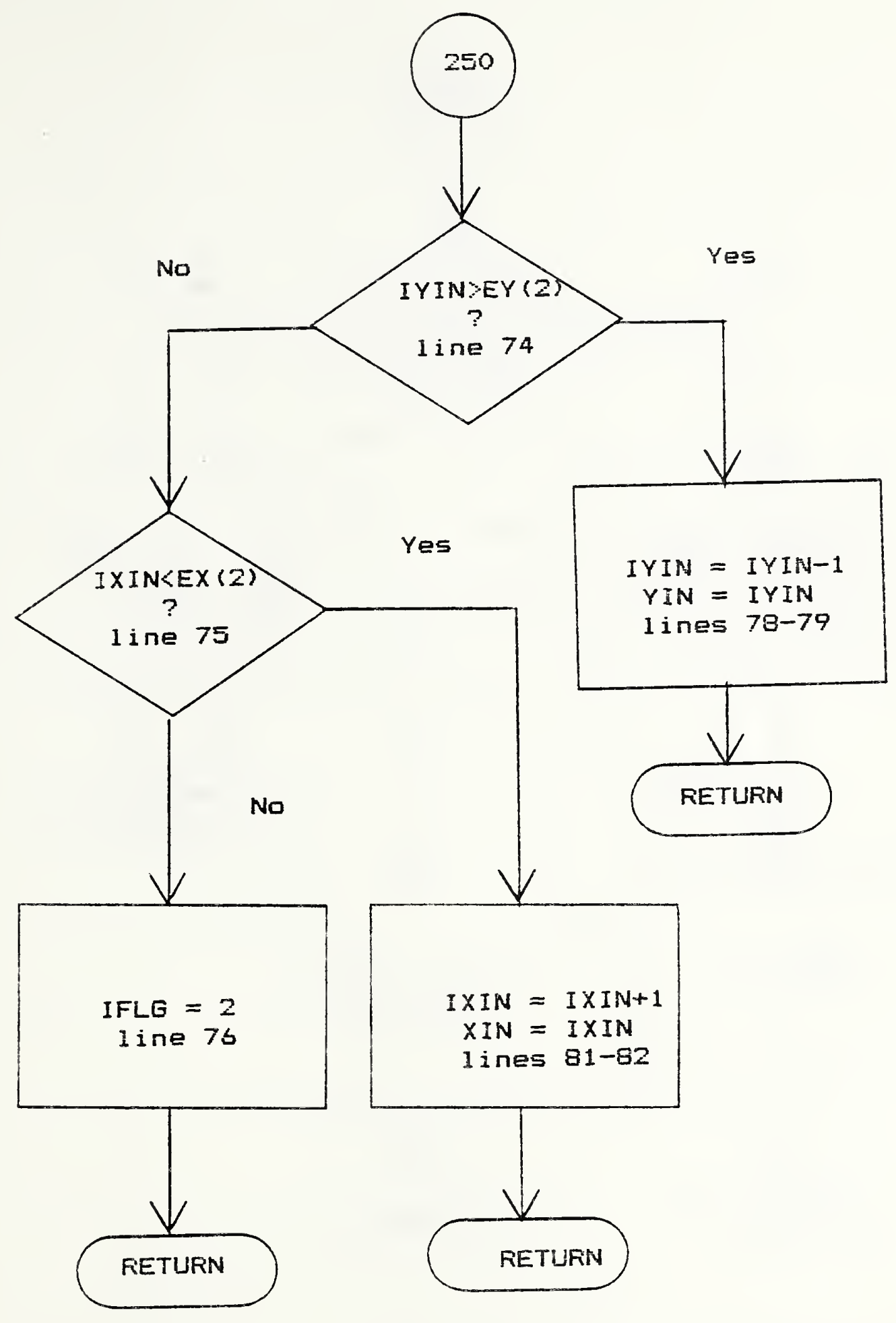




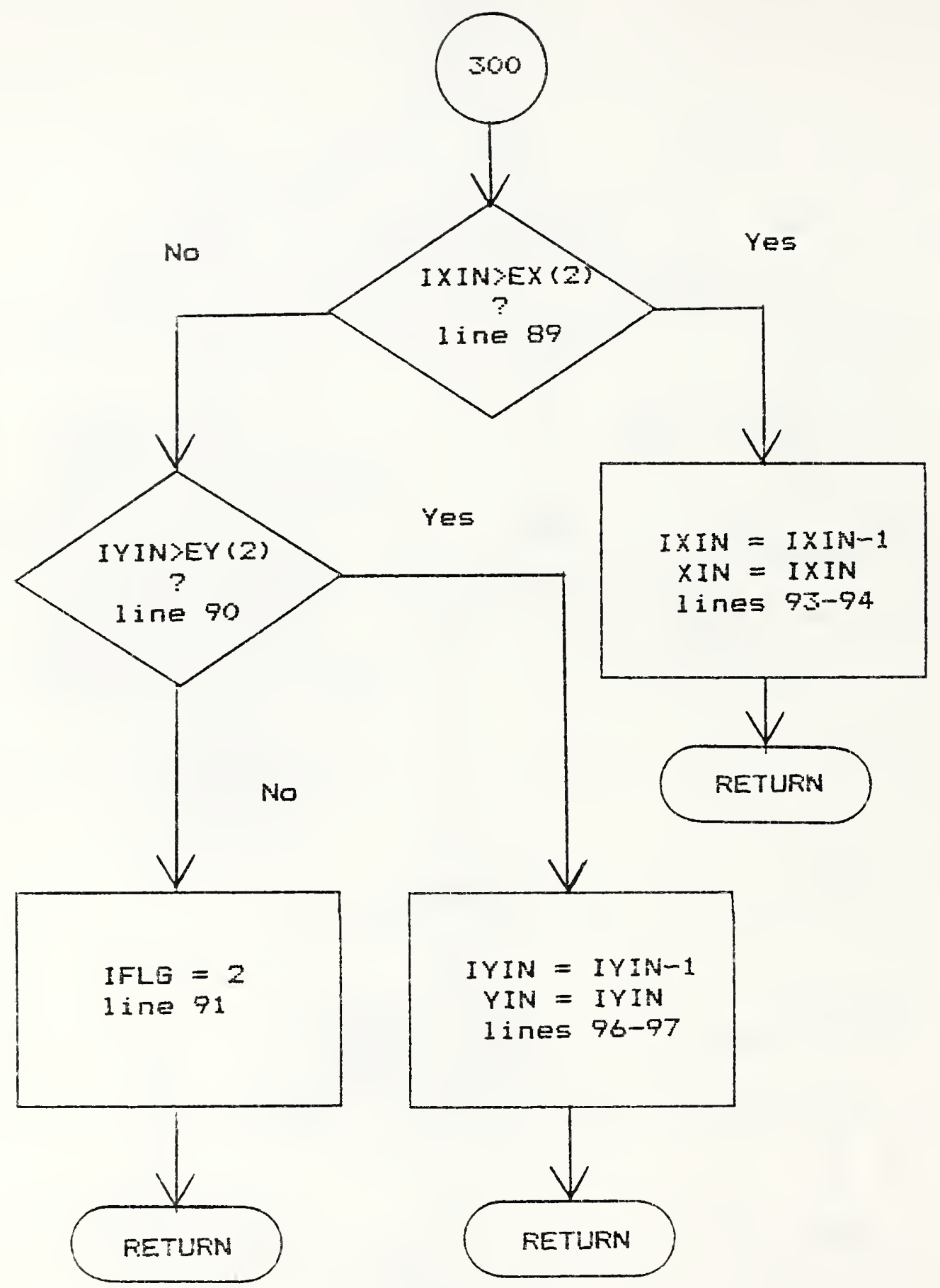




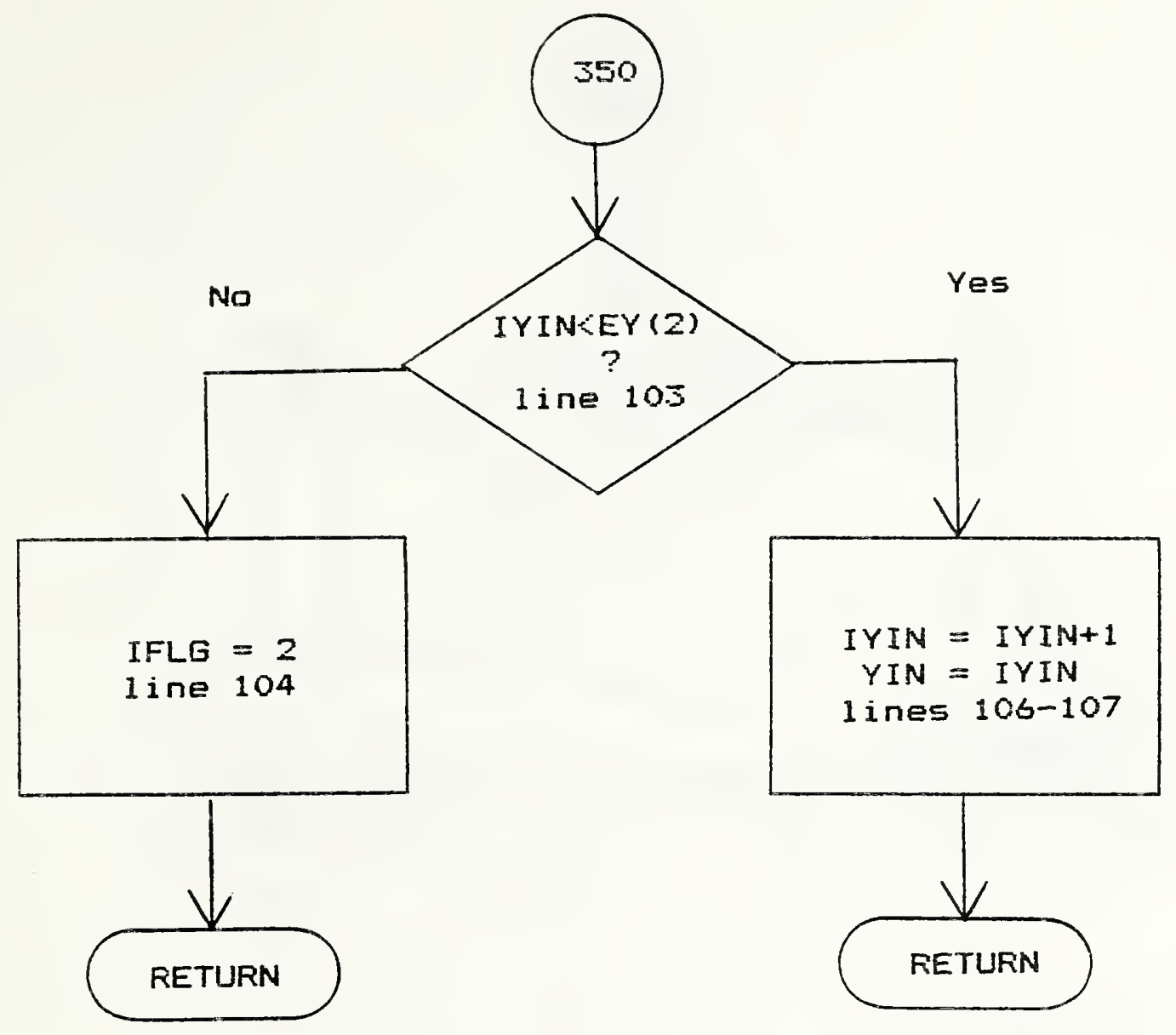




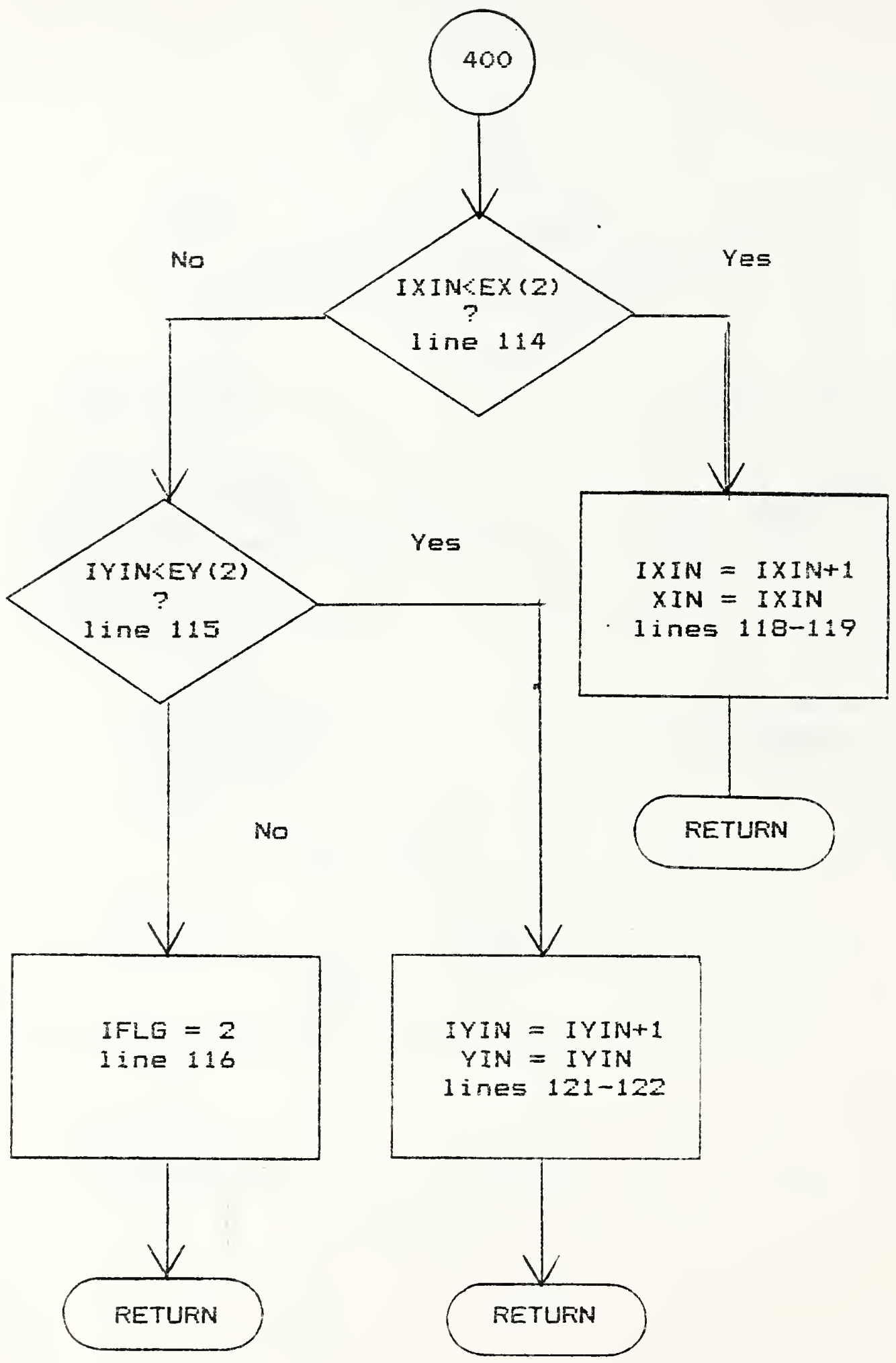




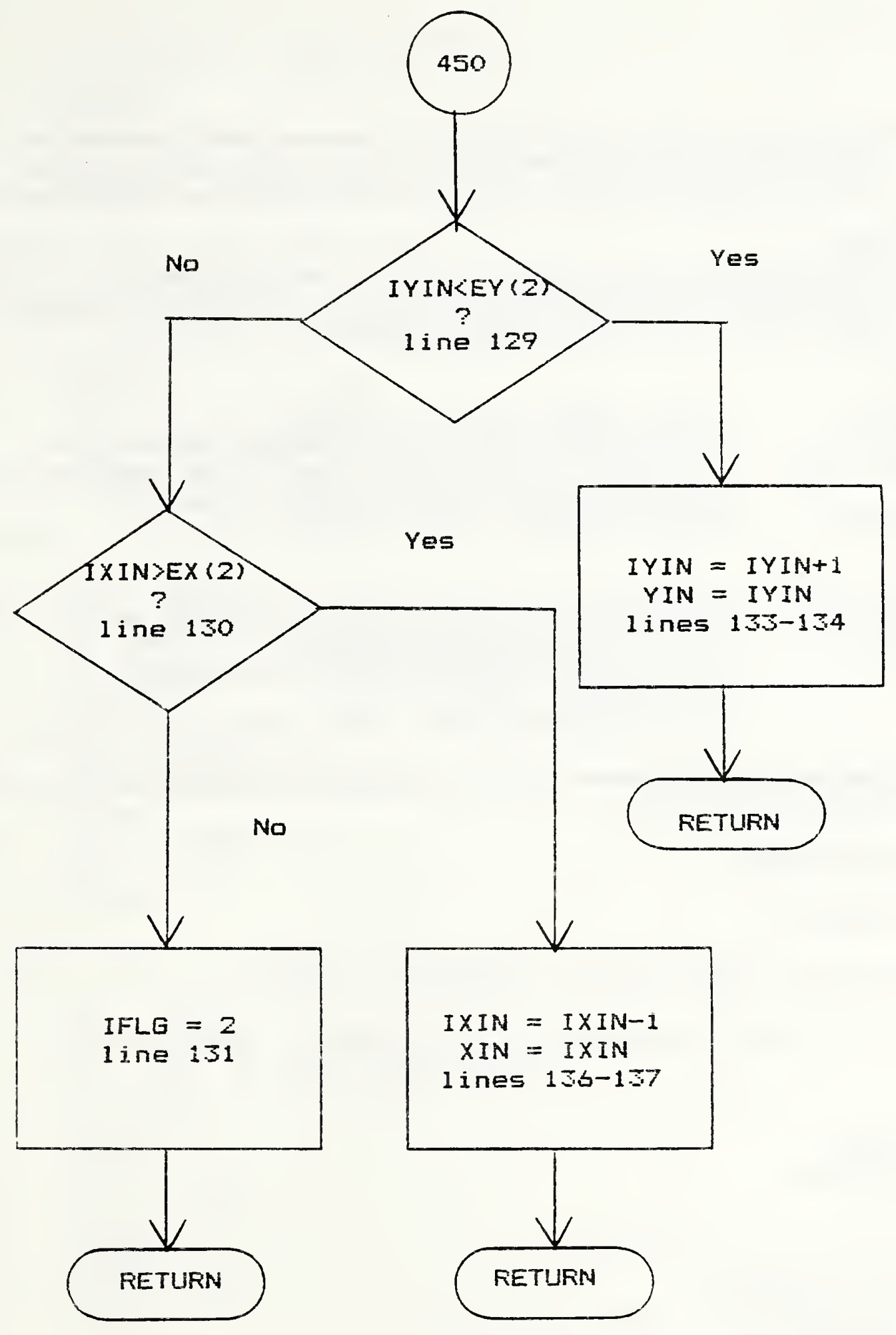


1

2

3

4

5

6

22

23

24

25

26

30

37

39

39

40

41

42

43

44

45

46

4.7

48

49

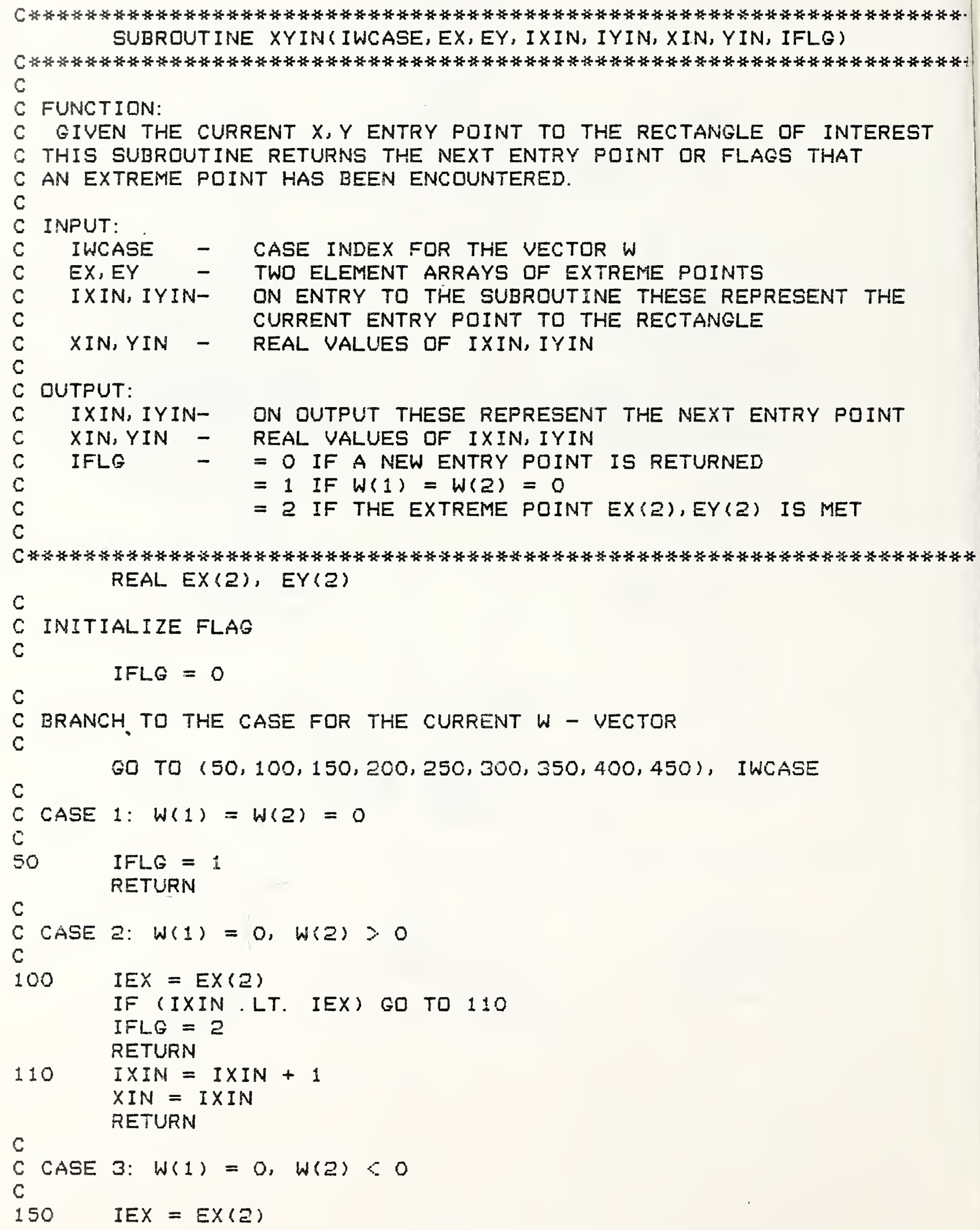


61

62

63

64

65

66

67

68

69

70

71

72

73

74

75

76

77

78

79

80

81

82

83

84

85

86

87

88

89

90

91

92

93

94

95

96

97

98

99

100

101

102

103

C CASE 4: $w(1)>0, w(2)=0$

C

$200 \quad$ IEY $=$ EY (2)

IF (IYIN. GT. IEY) GO TO 230

$I F L G=2$

RETURN

$230 \quad$ IYIN $=$ IYIN -1

$Y I N=I Y I N$

RETURN

c

C CASE 5: $w(1)>0, w(2)>0$

c

250 IEX $=E X(2)$

$I E Y=E Y(2)$

IF (IYIN . GT. IEY) GO TO 285

IF (IXIN.LT. IEX) GD TO 290

IFLG $=2$

RETURN

285 IYIN = IYIN -1

$Y I N=I Y I N$

RETURN

$290 \quad$ IXIN $=I X I N+1$

$X I N=I X I N$

RETURN

C

C CASE 6: $W(1)>0, W(2)<0$

$c$

300 IEX $=E X(2)$

$I E Y=E Y(2)$

IF (IXIN. GT. IEX) GO TO 345

IF (IYIN. GT. IEY) GO TO 348

IFLG $=2$

RETURN

345 IXIN $=$ IXIN -1

$X I N=I X I N$

RETURN

348 IYIN $=$ IYIN -1

$Y I N=I Y I N$

RETURN

$c$

C CASE 7: $w(1)<0, w(2)=0$

$\mathrm{C}$

350 IEY $=$ EY $(2)$

IF (IYIN.LT. IEY) GO TO 360

IFLG $=2$

RETURN 


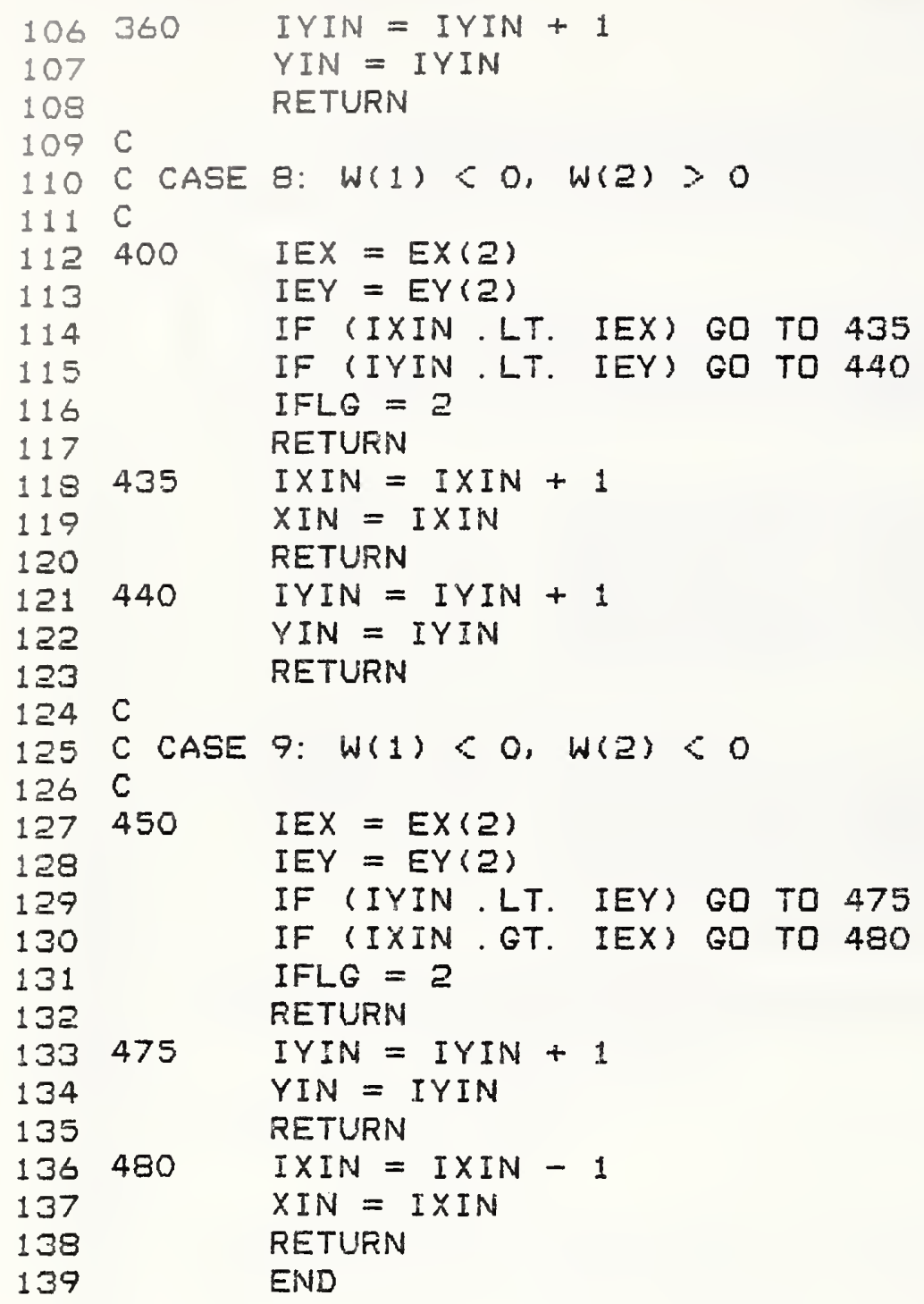


5. 19 Subroutine PUTFIL

5.19 .1 Summary

This subroutine opens a new file in mass storage and transfers an image of 512 records by 512 bytes per record. The user interactively specifies the file name for the new file prior to the subroutine opening it. The calling sequence for the subroutine is:

CALL FUTFIL (FCB, BUFFER, TABLE, CHANLS) -

The parameters passed are:

\begin{tabular}{|c|c|c|}
\hline FCB & - & $\begin{array}{l}\text { System Function Control Block. } \\
\text { INTEGER*2 Array }\end{array}$ \\
\hline BUFFER & - & $\begin{array}{l}\text { System buffer array. } \\
\text { INTEGER*2 Array }\end{array}$ \\
\hline TABLE & - & $\begin{array}{l}\text { Refresh Memory Channel to use: } \\
1,2 \text { or } 3 . \\
\text { INTEGER* } 2\end{array}$ \\
\hline CHANLS & - & $\begin{array}{l}\text { Bitmap for refresh memory } \\
\text { specified in TABLE. } \\
\text { INTEGER*2 }\end{array}$ \\
\hline
\end{tabular}

PUTFIL calls the following subroutines:

ZBUFF

IMAGE

SYSIO 
5.19.2 Flow Chart

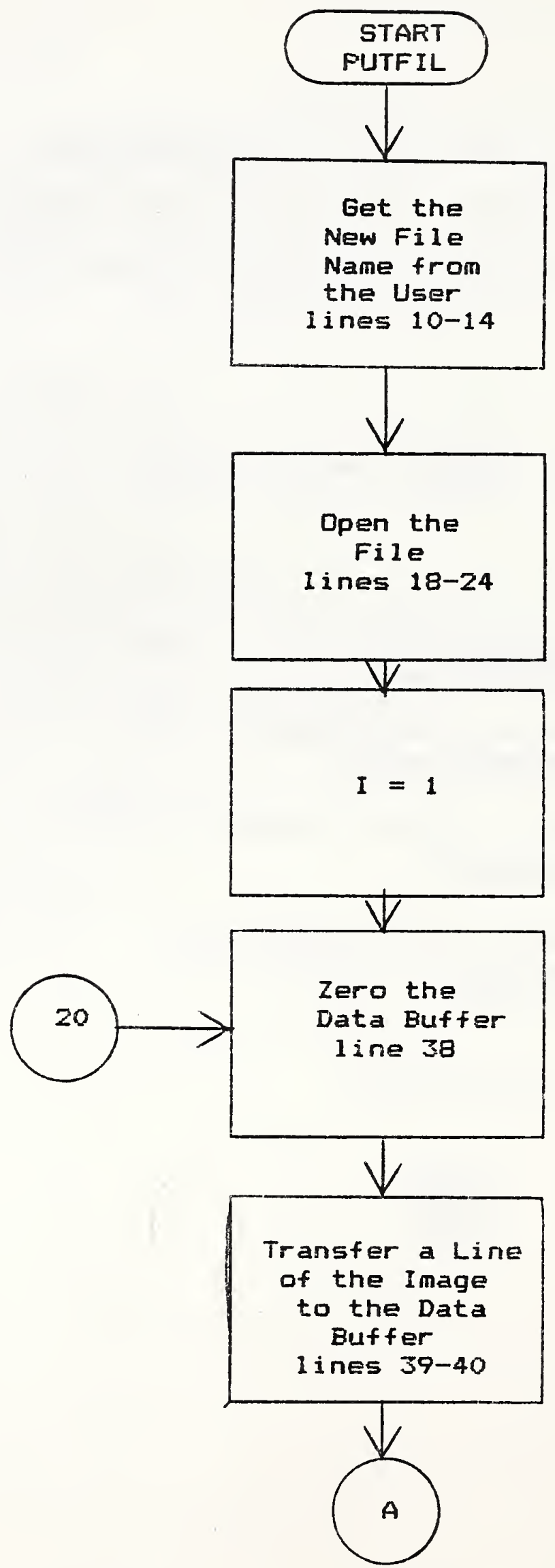




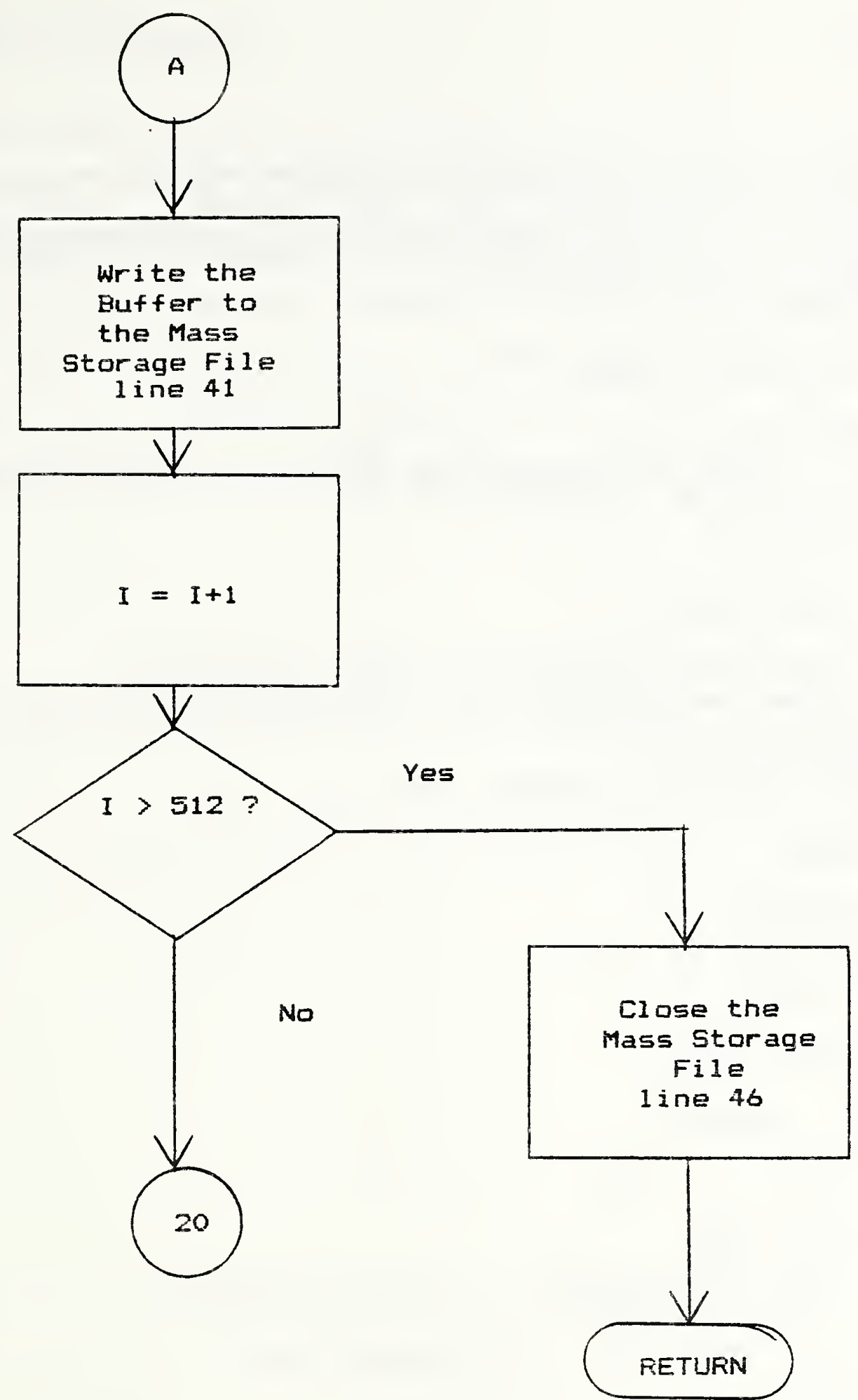


$1 C * * * * * * * * * * * * * * * * * * * * * * * * * * * * * * * * * * * * * * * * * * * * * * * * * * * * * * * * * * * * * * * * * * 4$

2 SUBROUTINE PUTFIL (FCB, BUFFER, TABLE, CHANLS)

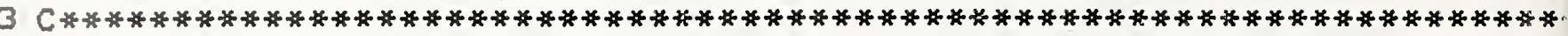

4 INTEGER*2 FCB $(2048)$, BUFFER (2048), CHANLS(16), TABLE(16)

5 INTEGER CHCODE, PBLK(B)

6 CHARACTER*16 FD

$7 \mathrm{C}$

Q C GET THE PICTURE FILE NAME

$9 \mathrm{C}$

10 WRITE $(5,10)$

1110

12

13

1411

1 "MAX OF 16 CHARACTERS. '?

FORMAT(" ENTER THE NAME OF THE FILE YOU WISH TO CREATE, '/

READ (5, 11) FD

$15 \mathrm{C}$

16 C OPEN THE FILE TO UNIT 4

$17 \mathrm{C}$

18

19

20

21

2212

23

2415

$25 \mathrm{C}$

$26 \mathrm{C}$ SET UP FILE SIZE

$27 \mathrm{C}$

28 NBYTES $=512$

29. NREC $=512$

$30 \mathrm{C}$

31 C GET THE CHANNEL CODE

$32 \mathrm{C}$

33

$34 \mathrm{C}$

35 C TRANSFER RECORD AT A TIME

$36 \mathrm{C}$

37

38

39

40

41

4220

OPEN (4, FILE=FD, STATUS= 'NEW', ACCESS= 'SEQUENTIAL',

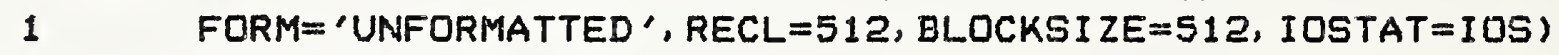
IF(IOS . EQ. O) GO TO 15

WR ITE (5, 12) IOS

FORMAT' ' IOSTAT ON OPENING THE FILE =', I4)

STOP

CONT INUE

$43 \mathrm{C}$

44 C CLOSE THE FILE

$45 \mathrm{C}$

46

47

DO $20 \quad I=1$, NREC

CALL ZBUFF (BUFFER, 2048)

CALL IMAGE (FCB, BUFFER, 0, I-1, NBYTES, 0, CHCODE, -1, 1, 1, 0, 1 $0,0,0,1)$

CALL SYSIO (PBLK, $\left.Y^{\prime} 38^{\prime}, 4, B U F F E R, N B Y T E S, O\right)$

CONTINUE

48

CLOSE (4)

RETURN

END 
6. A Acknowledgements

The author would like to thank Dr. E. Clayton Teague for encouraging the use of graphics techniques in surface quality studies and insisting that the earlier versions of this program should be made more user friendly. Mr. Saul Baker and Mr. Neal Webbier deserve credit for implementing one of those earlier programs. Dr. Theodore $V$. Vorburger contributed several helpful suggestions that helped make the exposition clearer.

199 
[1] Appel, A. Some techniques for shading machine renderings of solids, AFIPS Conference Proceedings, Spring Joint Computer Conference, Vol. 31 (1968), pp. 37-45.

[2] Crow, F. C. Shadow algorithms for computer graphics, Computer Graphics, Vol. 11, No. 2 (Summer 1977), $p p=355-361$.

[3] Dunhan, C. B. The necessity of publishing programs, The Computer Journal, Vol. 25, No. 1 (1992), pp. s1-62.

[4] Foley, J. D. and Van Dam, A. Fundamentals of Interactive Computer Graphics, Addison-Wesley Publishing Co., Reading, Mass., 1982 .

[5] Goldstein. R. A. and Nagel, R. 3-D visual simulation, Simulation, January 1971 , pp. 25-31.

[6] Teague, E. C., et. al. Three-dimensional stylus profilometry, Wear, Vol. 83 (1982), pp. 1-12.

[7] Webber, R. L. and Nagel, R. N. Three-dimensional enhancement of twa-dimensional images, Jour. Clinical Eng., Vol. 5, No. 1 (Jan.-Mar. 1980), pp. 41-50.

[8] Wyle, C.s et. al. Half-tone perspective drawing by computer, AFIPS Conference Proceedings, Fall Joint Computer Conference, Vol. 27 (1967), pp. 49-5a. 
APPENDIX A

IMAGE PROCESSOR FUNCTIDNS

\section{A. 1 Summary of Operations}

Image data can be transferred from the host computer to any one of three refresh memories either directly or by way of an input function memory. See Figure A1. The task of the input function memory is to directly control the scaling of data in order to ensure that it falls within the $0-255$ range, or 8 bits. Once in the refresh memory, it remains there unchanged. Actual image processing is performed by, controlling the individual pipeline processor channels, the feedback unit, the histogram generator and the graphics channel.

The individual pipeline processors contain several hardware capabilities. See Figure A2. Along with the ability to scroll an image and change magnification through the zoom hardware, the user may transform the image signals by loading the look-up tables and the output function memory. These, along with the Min-Max register, the constant register and range register, give the user several ways to control the image output to the monitor.

\section{A.2 Some Detailed Capabilities}

\section{A.2.1 Input Function Memory}

This is a host programmable look-up table that is applied to the data as it is transferred to a refresh memory or graphics memory, both from the host or during an image feedback operation. It is an optional look-up tabie and can be bypassed if the 
programmer so chooses. It is used to compact data of up to 13 bits to numbers of 8 bits or fewer and speeds up processing by not requiring data to be scaled in the host computer.

\section{A.2.2 Refresh Memory}

Each refresh memory consists of $512 \times 512 \times 8$ bits of random access data storage. This allows the host computer to access any pixel (or bit within a pixel) randomly. Images may be read or written vertically or horizontally by incrementing the location addresses either by rows or columns.

\section{A.2.3 Pipeline Processor Channel}

The three parallel pipeline processing channels can perform array arithmetic for each of the three primary colors. Any refresh memory channel for any combination of refresh memory channels) can be assigned to any of the pipelines (which in turn supply the RGB primary color). The pipelines can add, subtract, multiply and divide image data at real-time rates. The internal capabilities of the pipeline processors will be detailed below.

\section{A.2. .. 1 Pipeline Look-Up Tables}

Three look-up table memories are provided with each pipeline channel, giving a total of nine. One look-up tabie in each pipeline channel affects its associated refresh memory. These look-up tables (LUT's) are one of the two programmable processing elements following the refresh memories. The data for the LUT's is loaded by the host computer. The tables are used to implement 
the four basic arithmetic frocesses at real-time rates as well as affect image contrast.

\section{A.2.3.2 The Adder Array}

This takes the two"s complement sum of the look-up table outputs. Three sets are available, one for each primary pipeline.

\section{A.2.3.3 Dutput Function Menory}

Each pipeline contains an output function memory which transforms the outputs of the range registers to generate the final red, green and blue data streams.

\section{A.2.3.4 Min-Max Registers}

The Min-Max registers examine the data stream as it emerges from the adder array and determines the dynamic range of the data by finding the minimum and maximum pixel values. These registers are read by the host computer and are used in determining how to set the range register to process the data by the output function memory.

\section{A.2.3.5 Range and Constant Registers}

The range registers are used to reduce the data strean from the adder array to a stream for the output function memory. The constant register allows the addition and subtraction of a constant from the data stream before it enters the range register. 
This allows magnification by way of pixel replication of the displayed image by a factor of 2, 4 or 8 around an arbitrary location. The specification of the center point of the area to be magnified and the magnification factor is accomplished from the host computer. Zoom is nondestructive, in that the original data in the refresh memory is not destroyed.

\section{A.2.4 Color Monitor}

This monitor provides both full color and monochrome presentation.

\section{A.2.5 Graphics Refresh Memory}

This memory consists of five $512 \times 512$ one-bit graphics overlay planes. They are treated as an additional refresh memory for the purposes of reading and writing from the host computer. The graphics data, along with the cursor data stream, are fed to the graphics multiplexor. Under program control, this multiplexur can select between displaying graphics or graphics with cursor superimposed.

\section{A.2.6 Programmable Cursor}

The host computer can command the cursor position or read back the cursor position at any time. The cursor can be displayed with a constant intensity or bliniced. The host computer can also link the cursor position to the trackball unit. 


\section{A.2.7 Trackiali}

The trackball is used to selectively control the $x-y$

position of the cursor on the monitor screen. It is designed to allow the user to move the cursor in one pixel increments. Four function buttons are provided on the trackball housing. When pushed, the buttons indicate a state change to the host. These states are stored in a register that can be read by the host computer.

\section{A.2.8 Color Assignment Function Memory}

This assigns one of the possible 32,768 colors to each graphics plane and dynamically changes the assigned colors under programmatic control as the graphics planes overlay each other. The host computer can program the graphics colors by loading a map into the color assignment function memory. This map defines What color is to be dispiayed when any one graphics plane is on and also defines a different color to be displayed for each of the possible graphics plare combinations. The ability to dynamically change color assignments for overlapping regions guarantees that each graphics overlay can be distinguished from other graphics overlays at all times.

\section{A.2.9 Histogram Generator}

This unit is sometimes called a videometer and is a processing unit that rapidly computes the grey level histogram of the processed data streams just prior to their conversion to 
video signals at the output of a pipeline. It can generate the histogram of the entire image or of a defined subarea of the i mage.

\section{A.2.10 Feedback}

Except for the image data scaling performed by the Input Function memory, various transformations performed in the hardware do not actually modify the image data which is stored in the refresh memory. If the user wishes to retain the actual processed image data, it may be transferred back by the feedback unit to a refresh memory by way of the Input function memory. This capability allows the pirocessor to perform iterative operations on an image. 


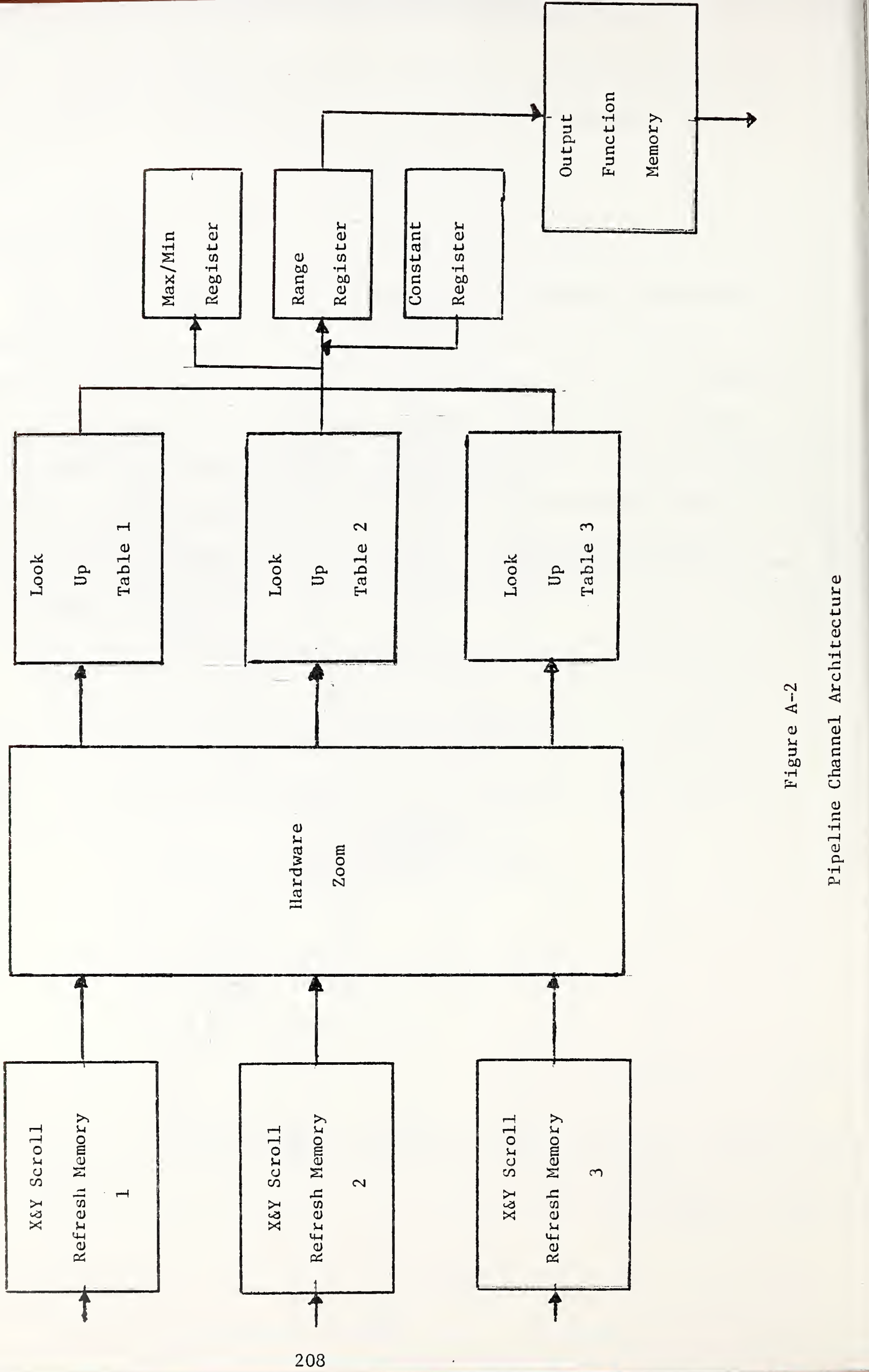


APFENDIX B

SYSTEM SUPFORT PFIGGRAMS

This appendix is devoted to 1 isting the names, functions, caliing sequences and the relevant comment portions of the system specific source programs used in the solid generation program. These programs are not available for public use and depend on the architectures of the image processor and the host computer facility. This section is presented so that anyone desiring to implement the SOLID program can understand the functions performed by the various calls not fully documented in this volume. The subroutine calls are divided into image processor subroutines and host computer subroutines.

\section{B.1 Image Frocessor Subroutines}

B.1.1 Subroutine BCHAN

This subroutine blanks an image shannel. It is used to turn off a channel link to the monitor.

SUBROUTINE ECHAN (FCB, BLFFER, CHCDDE, EITFLN)

ROUTINE TO SLANK IMAGE CHANNELS

CHCODE = BIT MAF FOR CHANNELS TO BE BLANKED

INTEGER CHCDDE, BITFLN

INTEGER VRSION

INTEGER *2 FCB (1), BUFFER (1) 
This subroutine reads or writes the cursor control register. This register is used to enable or disable the cursor.

SUBROUTIAE CRCTL (FCB, ON, RATE, LINKX, LINKY, BUTTON, BEEF; 1 MOVE, VRTETC, READ)

SUBROUTINE READS DR WRITES THE CUFSOR CONTROL REGISTER.

ARGUMENT DECLARATIONS:

INTEGER ON, RATE, LINKX, LINKY

INTEGER*2 FCB (1)

INTEGER VRTRTC, READ, MOUE, BEEP , BUTTON

ARGUMENT DESCRIPTIONS:

ON - O TURNS CURSOR OFF, 1 TURNS CURSOR ON

RATE - O CURSOR STEADY,

1 FAST BLINK.,

2 MEDIUM BLINK,

3 SLOW BLINK.

LINKX - O CURSOR STATIUNARY IN THE X DIRECTION,

1 ELRSOR $X$ FOSITION CONTROLLED BY TRACKBALL

LINKY - O CURSOR STATIONARY IN THE Y DIRECTION,

1 CURSOR Y FOSITIDN CONTFOLLED BY TRACKEALL

BEEP - $\quad 0 \Rightarrow$ ENABLE BEEFER, $1 \Rightarrow$ DISABLE BEEFEF

MOVE - $0 \Rightarrow$ NO MDVEMENT, $1 \Rightarrow$ CLRSOR HAS MOVED (READ ONLY)

BUTTON - BUTTON WORD (READ ONLY)

READ - $O$ IMFLIES WRITE, 1 IMPLIES READ. 
B.1.j Subroutine DADRS

This subroutine converts display channel numbers to display channel masks. A channel mask represents a 1 in a register bit that addresses the desired refresh menory.

SUBFUUTINE DADRS (CHMASK, CHANNO, CHCODE, NEANDS)

INTEGER CHCODE, NBANDS

INTEGER*2 CHMASK(1), CHANNO(1)

SUBROUTINE TO CONVERT DISPLAY CHANAEL NUMEERS (O THRU 1S)

TO DISPLAY CHANNEL MASKS (A 1 IN THE COFRESFONDING BIT)

CHMASK - INTEGER ARRAY IN WHICH DISPLAY CHANNEL MASKS AFE RETURNED

CHANNO - INTEEER ARRAY CONTAINING DISFLAY CHANNEL NUMEERS TO BE CONVEFTED

CHCODE - INTEGER MASK WHICH IS THE LOGICAL OF DF ALL DISPLAY CHANNEL MASKS

NEANDS - NUMBER OF DISPLAY BANDS 
This subroutine turns or the programmable cursor and defines its shape.

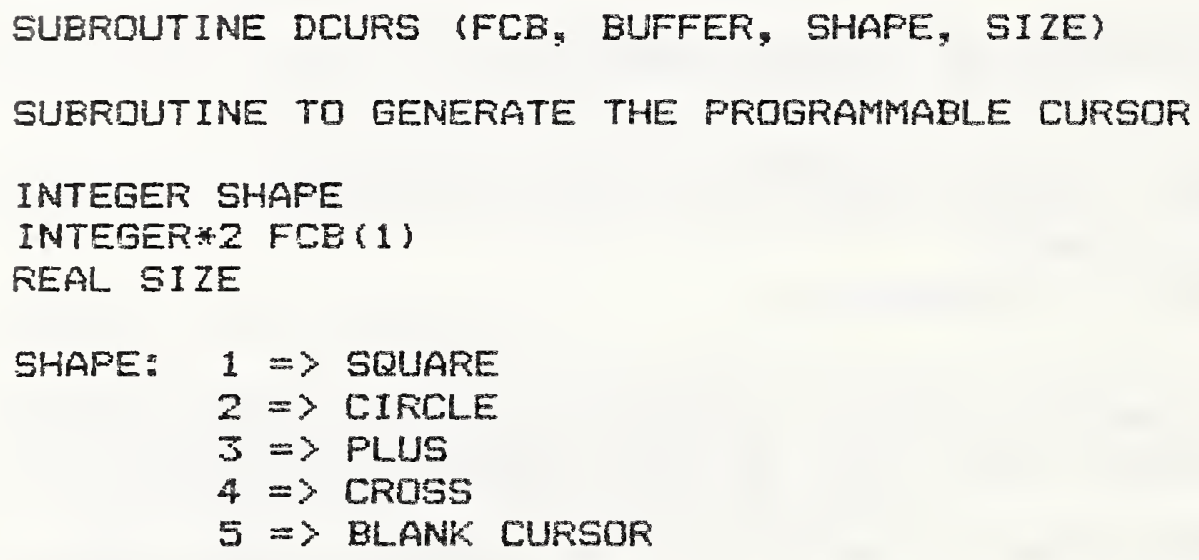

SIZE: PARAMETER DEFINING THE SIZE DF THE CORRESPONDIHG CURSOR SHAPE. SQUAFE = HEIGHT, CIRCLE = DIAMETER, FLUS = HEIGHT, CROSS = HEIGHT.

B. 1.5 Subrautine DEXEC

This subroutine clears the Function Control Block of all commands.

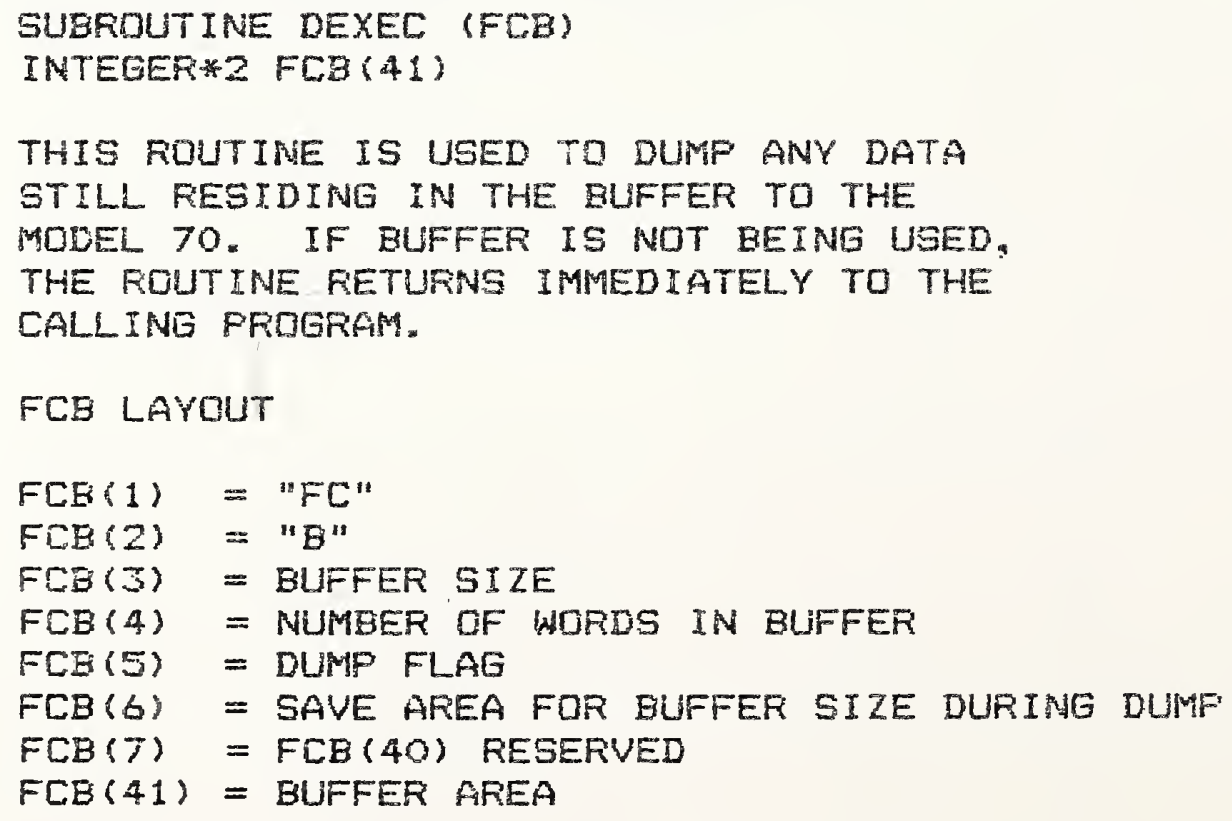




\section{B.1.6 Subroutine DFLUS}

This subroutine is used to draw a plus mark at a specified point in the graphics memory.

GUEROUTINE DPLUS (FCB, BLFFEF, CHATIN, FLAHES, $x, y$, SIZE)

INTEGER CHANNL, PLANES, $X, Y$, SILE

INTEGEF*2 FCB (1), EUFFEF(1)

SUBFOUTINE TO WFITE A PLUS AT $(X, Y)$ FOSITION

PARAMETEFS:

CHANNL - MASK OF CHANNELS TO WRITE
PLANES - MASK OF BIT FLANES TO WRITE
$X$ - X FOSITION (O REL)
$Y$ - Y POSITION (O FEL)
SIZE - WIDTH OF PLUS

B.1.7 Subroutine DUNIT

This subroutine initializes the look-up tables for the channei specified and sets various registers needed in orier to display an image.

SUEROUTINE DUNTT (FCE, BUFFEF, CHANLS, NCHAN, LEVELS)

THIS RDUTINE REESTAELISHES THE DISFLAY ENVIFINMENT FEQUIFED IU OFDER TO DISFLAY THE CONTENTS OF THE FEFFESH MEMORIES WITHOUT ANY FADIOHETFIC CHANGES.

GLOBAL VARIABLES

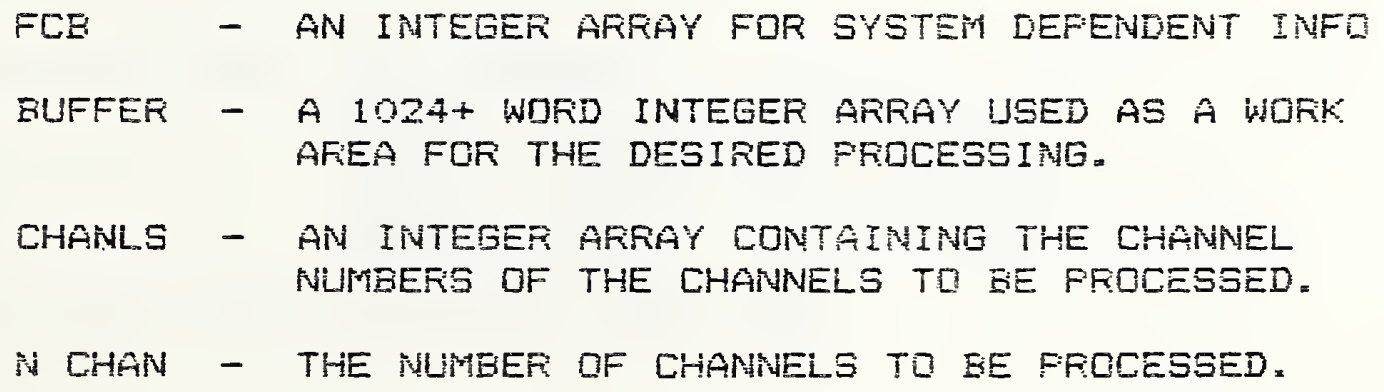


LEVELS - THE NUMBER DF QUANTIZATION LEVELS

FOR WHICH THE REFRESH MEMORIES ARE CONFIGURED. FOF $G$ GIT MEMORIES, LEVELS $=2 * * 8=256$.

INTEGER*2 FCB (1), BLFFER (1), CHANLS(1)

INTEGER NCHAN, LEVELS

B.1.8 Subroutine DVECT

This subroutine is used to draw a line between two points in tine graphics memory.

SUBROUTINE DVECT (FCB, $X 1, Y 1, X 2, Y 2$, CHCODE, 1 PLCDDE, BUFFEF)

USED TO DRAW A LINE BETWEEN THE POINT ( $X 1, Y 1)$ AND THE POINT $(\times 2, Y 2)$.

INFUTS:

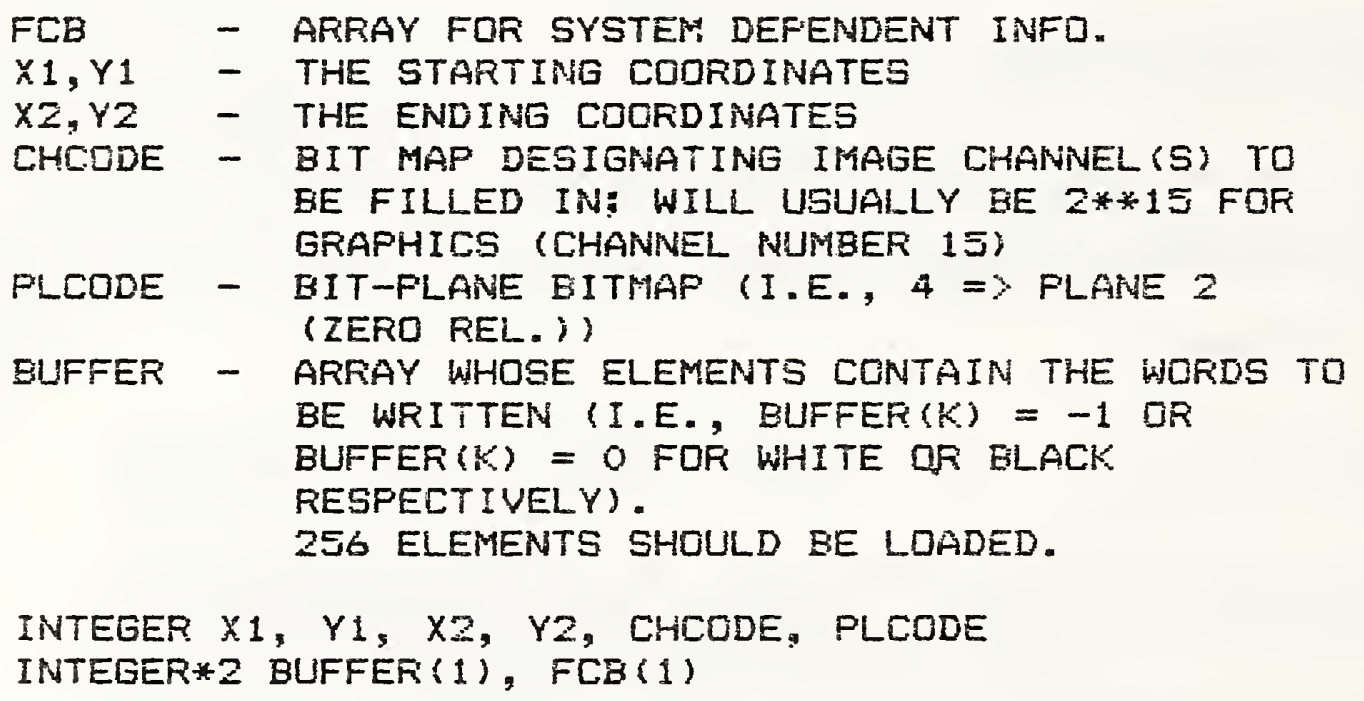


E. 1.7 Subroutine GRAFE

This subroutine controls any input and output to the graphics control registers. Its function is to enable or disable the graphics display.
SUEROUTINE GRAFE (FCB, DCURSR, DUIDEO, DGRAFH, ELOTCH, STATUS, 1 STVID, VFTETC, READ;

SUEFDUTINE WFITES THE GRAFHICS CONTEOL. FEGISTER.

ARGUHENT DECLAFATIONS:

INTEGER FEAD, STVID, VRTRTC

INTEGEF*2 FCB (1)

INTEGER DCUFSR, DUIDEO, DGFAPH, BLDTCH, STATLS

\section{ARGUMENT DESCRIFTIONS:}

DCURSR - DISABLES CURSOR DPTIGN AND SWITCHES IN GRAFHICS FLANE 7 DVIDEO - UNCONDITIONALLY TUFNS SCREEN BLACK

DGRAFH - TUFNS OFF ALL GRAFHICS CAFABILITY INCLUDING CURSGF

BLOTCH - SELECT BLOTCH FLANE

STATUS - SELECT STATUS PLANE

STVID - SETS STATUS VIDEQ ON

READ - READ GRAPHICS REGISTER WHEN SET

\section{E.1.10 Subroutine IMAGE}

This subroutine writes data from the host computer to $\equiv$ refresh memory or reads a refresh memory in order to transfer data to the host.

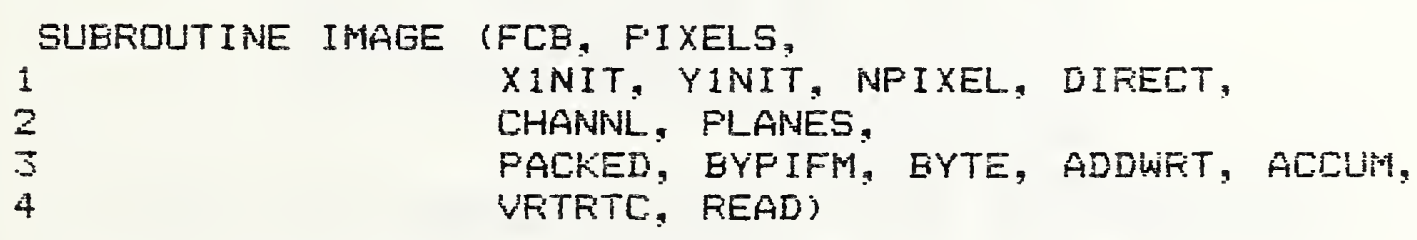

SUEROLTINE FEADS DR WFITES IMAGE DATA. 
INTEGER BURST, XINIT, YINIT, NPIXEL, DIFECT

INTEGER* 2 FCB (1), FIXELS(1)

INTEGER CHANNL, PLANES

INTEGER PACKED, BYPIFM, BYTE, ADDWRT, VIDOFD, ACCUM, VRTRTE, READ

ARGUMENT DESCFIFTIONS:

FIXELS - AN INTEGER ARRAY TO RECEIVE/CONTAIN THE IMAGE DATA

XINIT - THE X-COORDINATE DF THE FIRST PIXEL TRANSFERRED (O REL)

YINIT - THE Y-CODRDINATE OF THE FIRST PIXEL TFANSFERRED (O REL)

NPIXEL - THE TOTAL NUMEEF OF PIXELS TO TRANGFER

DIRECT - O IMPLIES READ/WRITE PROCEEDING TO THE RIGHT,

1 IMPLIES READ/WRITE FROCEEDING DOWNWAFD

CHANPL - A BIT MAP SELECTING THE CHANNEL (S) TO READ/WRITE:

$1 \rightarrow$ IMAGE $O$

$2 \rightarrow$ IMAGE 1

$4 \rightarrow$ IMAGE 2

ETC

$16.384 \rightarrow$ IMAGE 14

$-32768 \rightarrow$ IMAEE 15 (GRAPHICS)

WHEN WRITING ONLY, THESE CODES MAY BE COMEIMED

TD WFITE THE SAME DATA INTO TWO OR MORE CHANNELS.

FOR EXAMPLE, CHANML $=-32758$ WOLLD MEAN CHANNELS $1,3, \& 15$

PLANES - A BIT MAF SELECTING THE BIT PLANES TO FEAD/WRITE, NORMALLY -1, IE. ALL BITS. THE EXCEPTION TO THIS RULE IS WHEN WRITING IN THE GRAPHICS CHANNEL

PACKED - O IMPLIES 1 BYTE/WOFD, 1 IMPLIES 2 BYTES/WOFD

SYPIFM - O IMPLIES USE IFM, 1 IMPLIES BYPASS IFM

BYTE - $O$ IMPLIES NORMAL, 1 IMPLIES A PIXELS/BYTE, IE. BINARY DATA. **NOTE - X1NIT MUST BE A MULTIFLE DF $B$. ADDNRT - O IMPLIES NORMAL, 1 IMPLIES THAT THE DATA IN MEMDFY (S) IS ORE' ED TO THE DATA FRESENTED FROM THE COMPUTER AND THE RESULT IS STORED IN THE MEMORY (S). * NOTE - USED WHEN WRITING ONLY!!

ACCUM - $O$ IMPLIES NORMAL TRANSFER, 1 IMFLIES 16 BIT ACCUPULATOR MODE.

* *NOTE - the chanNel Select of channl PARAMETER MUST BE SET TO SELECT BOTH THE LSB AND THE MSB. NOTE THAT THE LSE MUST BE IN AN EVEN LOCATION AND THE MSB MUST BE THE NEXT CHANNEL.

URTFTE - O IMPLIES WRITE ANYTIME.

1 IMPLIES WRITE DURING VERTICLE RETRACE ONLY. READ - O IMPLIES WFITE, 1 IMPLIES READ. 
This subroutine initializes the function Contral Block. See Section B.1.5 for the structure of the Function Contral Block.

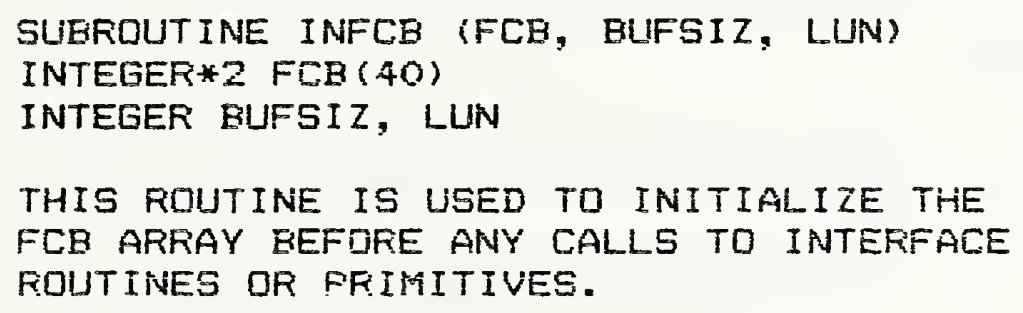

B.1.12 Subroutine LTCNT

This subroutine reads or writes to the look-up table masks in order to enable or disable them.

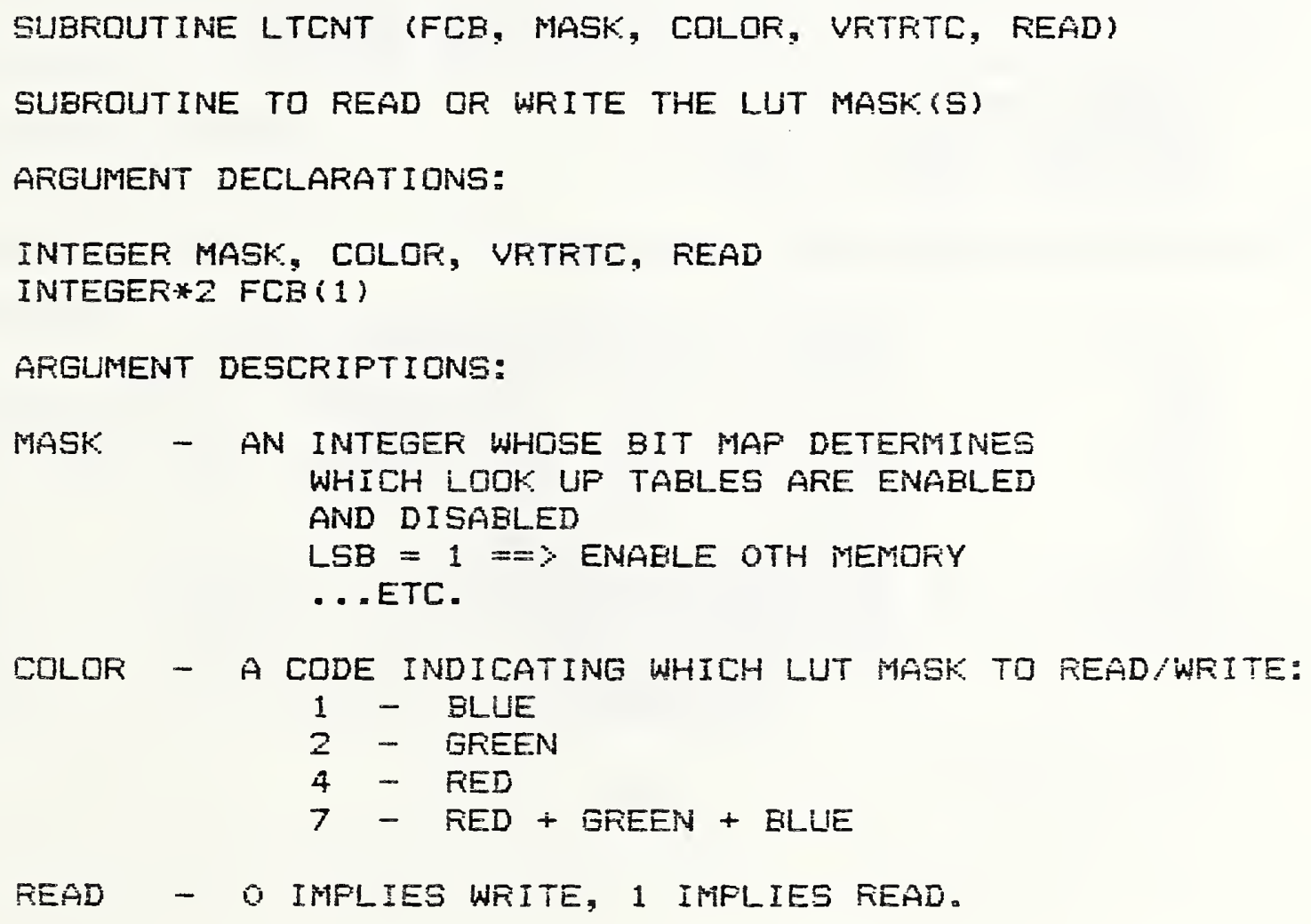


This subroutine sends a character from the host computer to the interface board in the image processor in order to clear the interface registers. This is done so that a new command can be sent from the hast.

SUEROUTINE MSTCL (FCB)

INTEEER *2 FCE (B)

\section{B.1.14 Subroutine DNCUR}

This subroutine turns on the cursor so that it may be displayed on the monitor.

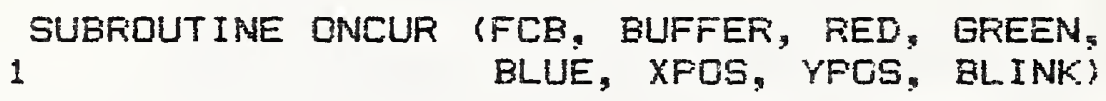

INTEGER XPOS, YFGS, BLINK

INTEGEF $* 2$ FCB (1), BUFFER (1)

REAL RED, GREEN, BLIUE

ROUTINE TO TURN ON THE CURSOR

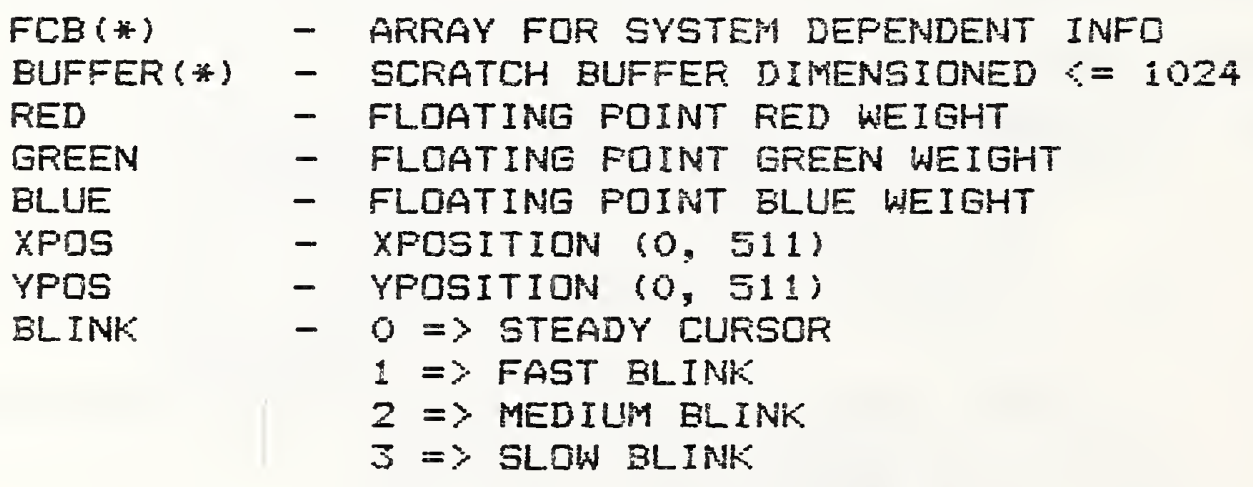

ALL WEISHTS MUST EE IN RANGE $0 .==>1$. 
This subroutine is used to read the location of the cursor. The viewer interacts with the image processor by pushing a button on the trackball housing. The image processor then $10 c a t e s$ the cursor.

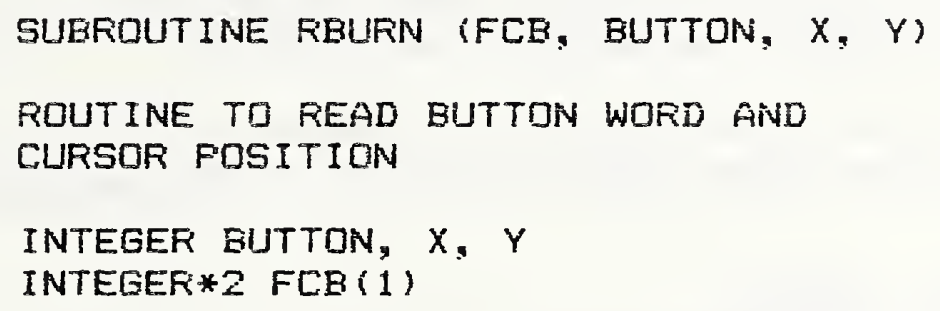

B.1.16 Subrautine STCOL

This subroutine is used to identify what colors should be displayed for each graphics bit plane. This does not enable or disable the planes.

SUBROUTINE STCOL (FCB, EUFFER, PLANE, FED, GFEEN, BLLE, INGEFT) SUBROUTINE TO SET COLOR OF GRAFHICS PLANES

INTEGER FLANE, INSERT

INTEGEF*2 FCB (1), BUFFEF $(1)$

REAL FED, GREEN, BLUE

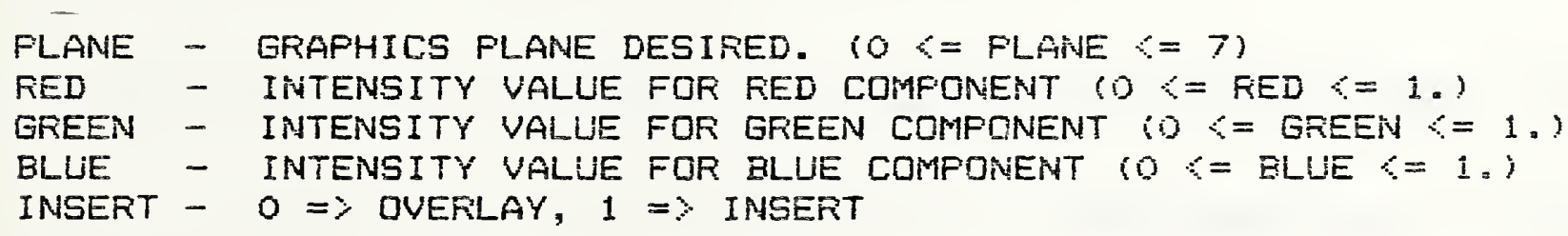


When several bitplanes have different colors and a graphics memory pixel is turned on for several bitplanes, then this subroutine defines what color should be displayed.

SUBROUTINE XCOLR (FCB, BUFFER, PLANE, INSERT)

THIS RIUTINE IS USED TO DEFINE THE COLDRS FOR AFEAS OF INTERSECTION BETWEEN GRAPHICS PLANES

A DISTINCT COLOR IS DBTAINED BY DOING AN EXCLUSIVE OR OF ALL THE COLOR WORDS CORRESPONDING TO THE INTERSECTING PLANES

INTEGER FLANE, INGEFT

INTEGER*2 FCB $(1)$, BLFFER (1)

PLANE - FLANE FOR WHICH WE ARE DEFINING THE INTERSECTIONS INSERT - OVERLAY HODE, INSERT $=1$, OVERLAY $=0$

B.1.18 Subroutine ZBUFF

This subroutine writes zeroes into a buffer specified by BUFFER. COUNT represents the number of zeroes written.

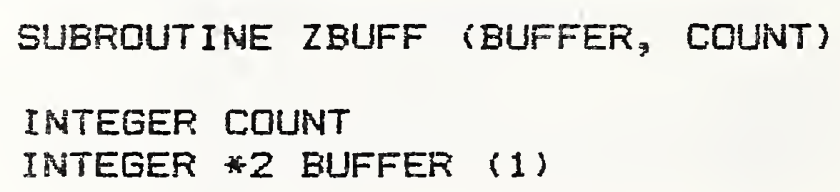

B. 2 Host Computer Subroutines

B.2.1 Subroutine ISBYT

This subroutine stores a byte from the low order position of one argument to any arbitrary position in another argument.

CALL ISBYTE (K, $N, N)$ 
where:

$$
\begin{aligned}
& K \text { - is the input argument whose least } \\
& \text { significant byte is to be stored. } \\
& \text { INTEGER }
\end{aligned}
$$

N - is the number of the byte in where storing takes place. INTEGER

\section{B.2.2 Subroutine SVC7}

This subroutine is used to correct an error in the FofTFin INQUIRE statement for the hast processor. It is a local correction only. The subroutine fetches the file control block for a specified file.

\section{CALL SVC7 (IFCB)}

where:

$$
\begin{aligned}
& \text { IFCB - INTEGER Array of at least } \\
& 6 \text { elements representing } \\
& \text { the file controi block. }
\end{aligned}
$$

B.2.3 Subroutine SYSIO

This subroutine performs input/output at the byte level.

CALL SYSIO (FBLK, FC, LU, STAFT, NBYTES, FANADE)

Arguments:

FBLK

is an INTEGER *4 array of at least five elements.

FE

is an INTEGEF* 4 argument that specifies the I/O function to be performed. 
is an INTEGER 4 arqumert that specifies the logical unit on which to perform $1 / 0$.

START

NEYTES

RANADD is any type of argument except character that specifies the starting address of the buffer used in the I/O transfer.

is an INTEGER*4 argument specifying the number of bytes to be transferred in this I/Q operation.

is an INTEGER*4 argument that specifies the $109 i c a i$ record number (starting at $b$ ) to be accessed on data transfer requests when bit 5 of $F C$ is set. This arqument should be a zero if randon I/O is not being used. 
AFPENDIX C

IMAGE DATA ACQUISITION

The image data used as input to the program documented in this report came from a three-dimensional profilometry instrument. Three-dimensional profilometry is the process of ottaining a topographic map of a surface from many parallel traverses of a stylus (See Teague, et.al. [6]). The number of data values required to represent a topographic map adequately can, depending on the spatial resolution desired, be as large as $0.25 \times 10^{\circ}$. This large amount of data poses a formidable pronlem in acquisition, processing, and displaying.

This problem can be surmounted with the use of a large minicomputer system interfaced to both a specially designed stylus stage and a raster graphics array processor and display unit. The electrical analog output of the stylus transiucer is converted into an intensity value at a corresponding point on the screen of a television monitor. A schematic diagram of the system for acquiring three-dimensional styius profilometry data and for displaying the data as an intensity image is shown in Figure $\mathrm{C1}$. The system is composed of a commercial stylus transducer, a precision $X-Y$ stage buiit at NBS and a $32-b i t$ minicomputer system with a core memory of 4 miliion bytes, a mass storage of $160 \mathrm{milli}$ on bytes and a raster graphics display unit which contains hardware for video rate memory refresh of a color television mmonitor and video rate iterative processing of data stored in the refresh memories (see fppendix A). 
As the stage moves the test specimen beneath a fixed stylus location, the electrical signal from the stylus transducer is converted to 12-bit digital values at an array of $512 x \equiv 12 x-y$ positions and stored on a disk. This is the normal array size, being controlled by the size of the graphics refresh memory size. Using the graphics display unit, the optimum 8 bits of the data are then selected for storage in the refresh memory and for display and processing. From this array of digital vaiues, an image of the topography is generated in which the intensity on the monitor screen is proportional to the surface height of the specimen at the corresponding surface location. Once the data are in refresh memory, a variety of transformations may be appiied to enhance visual perception of surface features. The program documented in this volume is one of them.

A schematic picture of the stage is given in figure c2. For mare details on the stage design, the reader is referred to the article by Teague, et.al. [b]. Mation in both axes is produced by stepping motors under control of the minicomputer. Position determination of the $X-Y$ stage is done by way of a commercial interferaneter system. Scanning areas cover approximately $1 \mathrm{~min}^{2}$. 

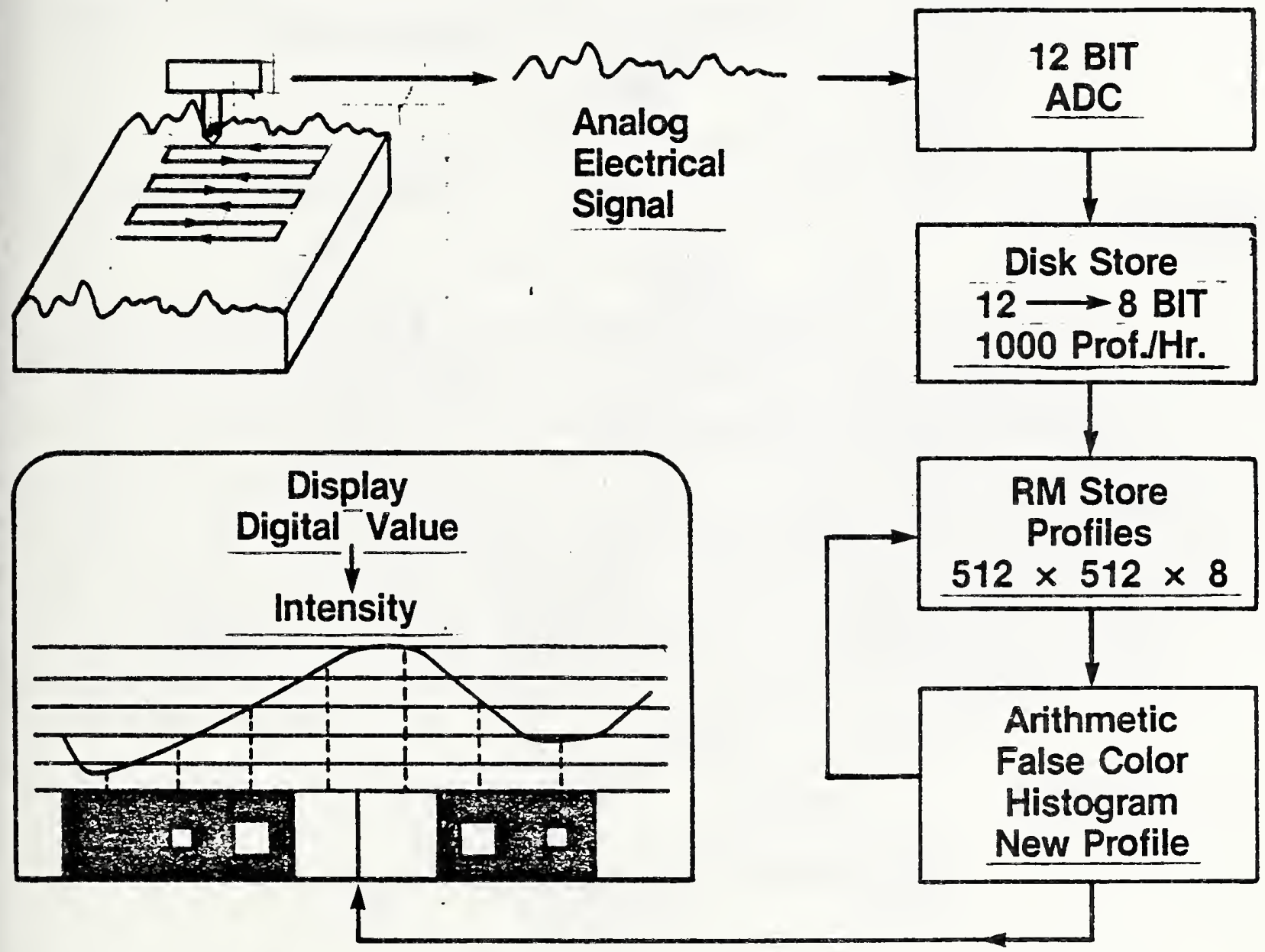

Figure C-1

Surface Data Transfer 


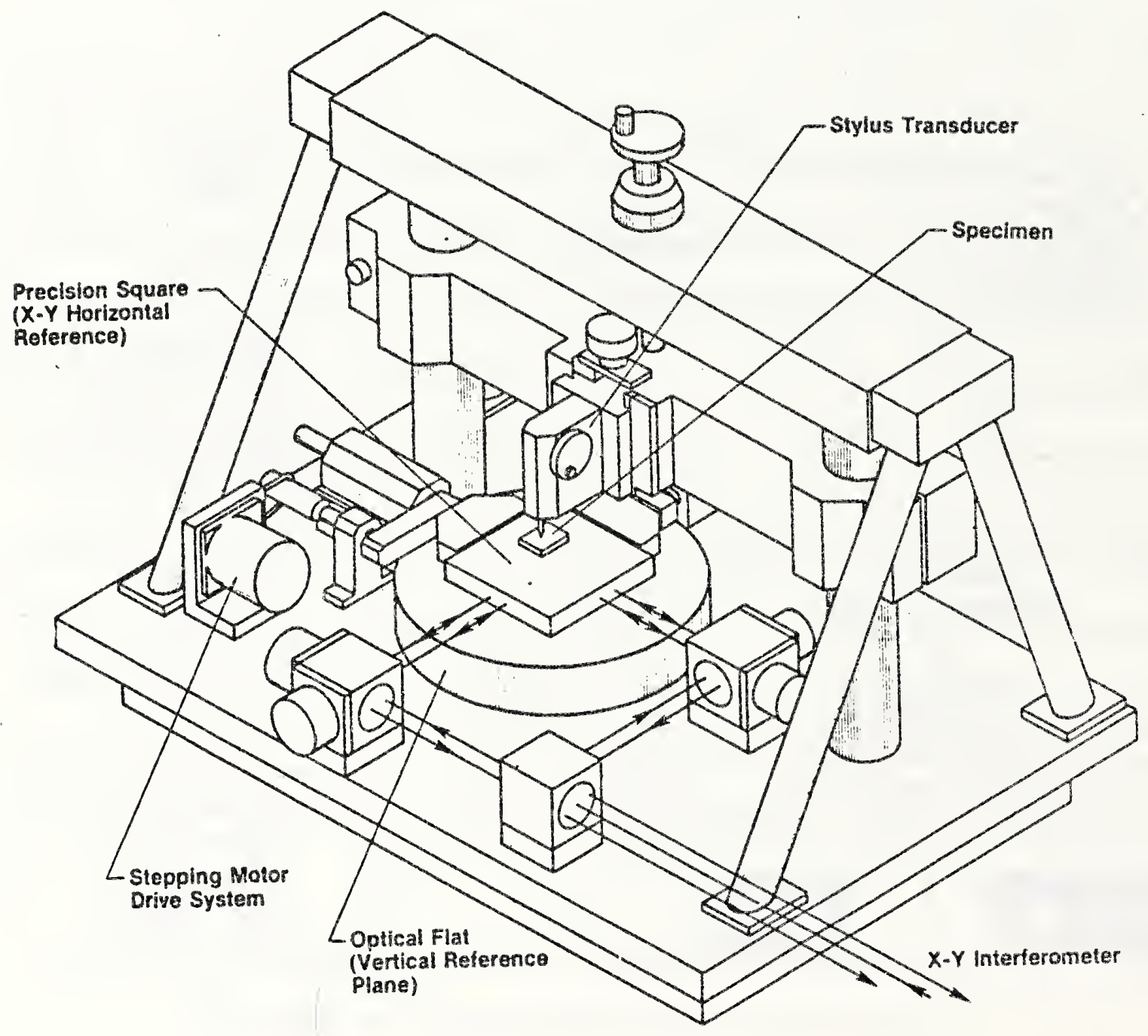

Figure $C-2$

Three Dimensional. Surface

Profilometer 
NBS-114A (REV. 2.8C)

U.S. DEPT. OF COMM.

1. PUBLICATION OR REPORT NO.

BIBLIOGRAPHIC DATA

SHEET (See instructions) NBSIR $85-3121$ 2. Performing Organ. Report No. 3. Publication Date June 1985

4. TITLE AND SUBTITLE

SOLID - A FORTRAN Program for Displaying Three-Dimensional Surface Topographies

5. AUTHOR(S)

David E. Gilsinn

6. PERFORMING ORGANIZATION (If joint of other than NBS, see instructions)

7. Contract Grant No.

NATIONAL BUREAU OF STANDARDS

DEPARTMENT OF COMMERCE

8. Type of Report \& Period Covered

WASHINGTON, D.C. 20234

9. SPONSORING ORGANIZATION NAME ANO COMPLETE ADDRESS (StTeet, City. StOte, ZIF)

10. SUPPLEMENTARY NOTES

Document describes a computer program; SF-185. FIPS Software Summary, is attached.

11. ABSTRACT (A 200-word or less factual summary of most significant information. If document includes a significant bibliogrophy or literoture survey. mention it here)

Graphic images of digital surface topography can be viewed as topographic maps. The intensities displayed on computer monitors represent surface height. Hidden surface graphics techniques can be used to project a solid projection of the surface on a high resolution computer monitor. The program documented here implements a multipurpose ray tracing algorithm to a) cast shadows, b) remove hidden surfaces, and c) project a solid image on a monitor. Although the algorithm is written in FORTRAN, its implementation depends on a unique inage processor. This report details the underlying geometry, program logic, and host computer-to-image processor interface requirements, and should enable a user to modify the program for a different image processor implementation.

12. KEY WOROS (Six to twelve entries; olphabetical order; capitalize only proper names; and separate key words by semicolons) graphics; hidden surface algorithm; image processing; ray tracing algorithm; surface topography; three-dimensional display

\section{AVAILABILITY}

X] Unlimited

$\square$ For Official Distribution. Do Not Release to NTIS

$\square$ Order From Superintendent of Documents, U.S. Government Printing Office, Washington, D.C. 20402.

[x] Order From National Technical Information Service (NTIS), Springfield, VA. 2216I
14. NO. OF PRINTED PAGES

\section{5}

15. Price

$\$ 20.50$ 
